\title{
A STUDY OF THE REMINERALIZATION OF ORGANIC CARBON \\ IN NEARSHORE SEDIMENTS USING CARBON ISOTOPES
}

\author{
by \\ ANN P. MCNICHOL \\ B.S., Trinity College, 1978 \\ SUBMITTED IN PARTIAL FULFILLMENT \\ OF THE REQUIREMENTS FOR THE DEGREE OF \\ DOCTOR OF PHILOSOPHY \\ at the \\ MASSACHUSETTS INSTITUTE OF TECHNOLOGY \\ and the \\ WOODS HOLE OCEANOGRAPHIC INSTITUTION
}

SEPTEMBER, 1986

๑Ann P. McNichol, 1986

The author hereby grants to MIT and WHOI permission to reproduce and distribute copies of this thesis document in whole or in part.

Signature of Author

Joint Program in Oceanography, Massachusetts Institute of

Technology and Woods Hole Oceanographic Institution, and the

Department of Earth, Atmospheric, and Planetary Sciences,

Massachusetts Institute of Technology

Certified by

Ellen R.M. Druffel and Cindy Lee, Thesis Supervisors

Accepted by

Edward Boyle, Chăr, Jolnt Committee for Chemical Oceanography, Massachusetts Institute of Technology/ Woods Hole Oceanographic Institution. 
A study of the remineralization of organic carbon was conducted in the organic-rich sediments of Buzzards Bay, MA. Major processes affecting the carbon chemistry in sediments are reflected by changes in the stable carbon isotope ratios of dissolved inorganic carbon $\left(\sum \mathrm{CO}_{2}\right)$ in sediment pore water. Six cores were collected seasonally over a period of two years. The following species were measured in the pore waters: $\Sigma \mathrm{CO}_{2}$, $\delta^{13} \mathrm{C}-\left[\mathrm{CO}_{2}, \mathrm{PO}_{4}, \sum \mathrm{H}_{2} \mathrm{~S}, \mathrm{Alk}, \mathrm{DOC}\right.$, and $\mathrm{Ca}$. Measurements of pore water collected seasonally show large gradients with depth, which are larger in summer than in winter. The $\delta^{13} \mathrm{C}(\mathrm{PDB})$ of $\sum \mathrm{CO}_{2}$ varies from $1.30 / 00$ in the bottom water to approximately $-100 / 00$ at $30 \mathrm{~cm}$. During all seasons, there was a trend towards more negative values with depth in the upper $8 \mathrm{~cm}$ due to the remineralization of organic matter. There was a trend toward more positive values below $8 \mathrm{~cm}$, most likely due to biological irrigation of sediments with bottom water. Below 16-20 cm, a negative gradient was re-established which indicates a return to remineralization as the main process affecting pore water chemistry.

Using the $\left[\mathrm{CO}_{2}\right.$ depth profile, it was estimated that $67-85 \mathrm{gC} / \mathrm{m}^{2}$ are oxidized annually and $5 \mathrm{gC} / \mathrm{m}^{2}-\mathrm{yr}$ are buried. The amount of carbon oxidized represented remineralization occurring within the sediments. This estimate indicated that approximately $20 \%$ of the annual primary productivity reached the sediments. The calculated remineralization rates varied seasonally with the high of $7.5 \times 10^{-9} \mathrm{~mol} / \mathrm{L}-\mathrm{sec}$ observed in August 84 and the low $\left(0.6 \times 10^{-9}\right)$ in December 83 . The calculated remineralization rates were dependent on the amount of irrigation in the sediments; if the irrigation parameter is known to $\pm 20 \%$, then the remineralization rates are known to this certainty also. The amount of irrigation in the sediments was estimated using the results of a seasonal study of ${ }^{222} \mathrm{Rn} /{ }^{226} \mathrm{Ra}$ disequilibria at the same study site (Martin, 1985). Estimates of the annual remfneralization in the sediments using solid-phase data indicated that the solid-phase profiles were not at steady-state concentrations.

The isotopic signature of $\left[\mathrm{CO}_{2}\right.$ was used as an indicator of the processes affecting $\left[\mathrm{CO}_{2}\right.$ in pore water. During every month, the oxidation of organic carbon to $\mathrm{CO}_{2}$ provided over half of the carbon added to the $\sum \mathrm{CO}_{2}$ pool. However, in every month, the $\delta^{13} \mathrm{C}$ of $\sum \mathrm{CO}_{2}$ added to the pore water in the surface sediments was greater than $-15 \% / 00$, significantly greater than the $\delta^{13} \mathrm{C}$ of solid-phase organic carbon in the sediments $(-20.6 \mathrm{o} / 00)$. The $\delta^{13} \mathrm{C}$ of $\left[\mathrm{CO}_{2}\right.$ added to the pore water in the sediments deeper than $7 \mathrm{~cm}$ was between -20 and $-210 / 00$, similar to the organic carbon in the sediments. Possible explanations of the ${ }^{13} \mathrm{C}$-enrichment observed in the surface sediments were:

a) significant dissolution of $\mathrm{CaCO}_{3}\left(\delta^{13} \mathrm{C}=+1.7 \mathrm{o} / 00\right)$

b) the addition of significant amounts of carbonate ion from bottom water to pore water

c) an isotopic difference between the carbon oxidized in the sediments

and that remaining in the sediments.

The effect of $\mathrm{CaCO}_{3}$ dissolution was quantified using measured dissolved 
Ca profiles and was not large enough to explain the observed isotopic enrichment.

An additional source of ${ }^{13} \mathrm{C}$-enriched carbon was bottom water carbonate ion. In every month studied, there was a net flux of $\sum \mathrm{CO}_{2}$ from pore water to bottom water. The flux of pore water $\mathrm{COO}_{2}$ to bottom water ranged from a minimum of $10 \times 10^{-12} \mathrm{~mol} / \mathrm{cm}^{2}-\mathrm{sec}$ in December 83 to a maximum of $50 \times 10^{-12} \mathrm{~mol} / \mathrm{cm}^{2}-\mathrm{sec}$ in August 84 . However, because the $\mathrm{pH}$ of bottom water was about 8 while that of the pore water was less than or equal to 7 , the relative proportion of the different species of inorganic carbon $\left(\mathrm{H}_{2} \mathrm{CO}_{3}^{*}, \mathrm{HCO}_{3}^{-}, \mathrm{CO}_{3}^{2}\right)$ was very different in bottom water and pore water. Thus, while there was a net flux of $\mathrm{CCO}_{2}$ from pore water to bottom water, there was a fiux of carbonate ion from bottom water to pore water. Because bottom water $\sum \mathrm{CO}_{2}$ was more ${ }^{1}{ }^{3} \mathrm{C}$-enriched than pore water $\mathrm{CCO}_{2}$, the transfer of bottom water carbonate ion to pore water was a source of ${ }^{13} \mathrm{C}$-enriched carbon to the pore water. If the $\delta^{13} \mathrm{C}$ of $\mathrm{CO}_{2}$ added to the pore water from the oxidation of organic carbon was $-20.6 \mathrm{o} / 00$, then the flux of $\mathrm{CO}_{3}^{2-}$ from bottom water to pore water must have been $10-30 \%$ of the total flux of $\mathrm{CCO}_{2}$ from pore water to bottom water. This is consistent with the amount calculated from the observed gradient in carbonate ion.

Laboratory experiments were conducted to determine whether the $\delta^{13} \mathrm{C}$ of $\mathrm{CO}_{2}$ produced from the oxidation of organic carbon $\left(\delta^{13} \mathrm{C}-O C_{0 x}\right)$ was different from the $\delta^{13} \mathrm{C}$ of organic carbon in the sediments $\left(\delta^{13} \mathrm{C}-\mathrm{SOC}\right)$. In the laboratory experiments, mud from the sampling site was incubated at a constant temperature. Three depths were studied $(0-3,10-15$, and $20-25 \mathrm{~cm}$ ). For the first study (IEI), sediment was stirred to homogenize it before packing into centrifuge tubes for incubation. For the second study (IE2), sediment was introduced directly into glass incubation tubes by subcoring. The second procedure greatly reduced disturbance to the sediment. Rates of $\mathrm{CO}_{2}$ production were calculated from the concentrations of $\mathrm{SCO}_{2}$ measured over up to 46 days.

In both studies, the values of $R_{c}$ in the deeper intervals were about $10 \%$ of the surface values. This was consistent with the field results, although the rates decreased more rapidly in the field. In all cases, the remineralization rates during the beginning of IEl were much greater than those at the beginning of IE2. The sediment for IEl was collected in February 84. The measured value of $R_{c}$ in the surface sediment of the laboratory experiment $\left(24 \times 10^{-9} \mathrm{~mol} / \mathrm{L}-\mathrm{sec}\right)$ was much greater than the value of $R_{c}$ observed in the field in another winter month, December $83\left(.62 \times 10^{-9}\right)$. The sediment for IE2 was collected in August 85 . The measured values of $R_{c}$ in the surface sediment $(6.6-12 \times$ $10^{-9} \mathrm{~mol} / \mathrm{L}-\mathrm{sec}$ ) were consistent with the field values from August 84 ( 7.5 $x\left(0^{-9}\right)$. The $\left[\mathrm{CO}_{2}\right.$ results indicated that IE2 reproduced field conditions more accurately than IEl did.

The isotopic results from the experiments strongly suggested that $\delta^{13} \mathrm{C}-O C_{0 x}$ in the surface sediments $(-17.8 \mathrm{o} / 00 \pm 1.9 \mathrm{o} / 00)$ was greater than $\delta^{13} \mathrm{C}-\mathrm{SOC}(-20.6 \pm 0.2 \mathrm{o} / 00)$. The magnitude of the observed fractionation was small enough that the observed values of $\delta^{13} \mathrm{C}-\mathrm{COO}_{2}$ in the pore waters could be explained by fractionated oxidation coupled with the diffusion of carbonate ion from bottom water to 
pore water. The observed fractionation was most likely due to the multiple sources of organic carbon to coastal sediments. A study of the natural levels of radiocarbon in these sediments indicated that the carbon preserved in the sediments is approximately $30 \%$ terrestrial while the rest is from phytoplankton. 


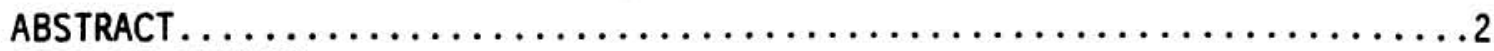

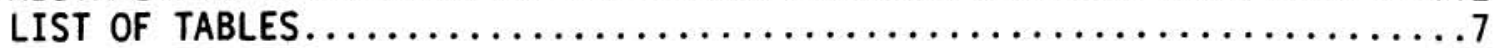

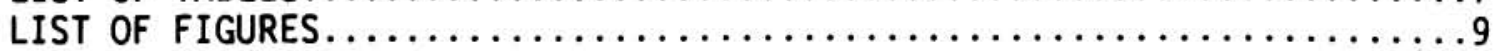

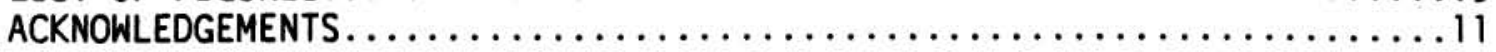

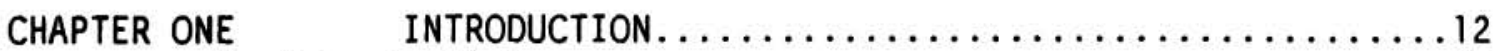

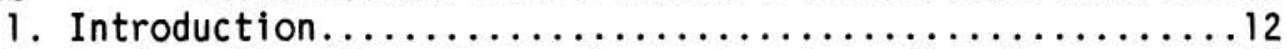

2. Remineralization of organic carbon in sediments.......15

A. Diagenetic models......................... 18

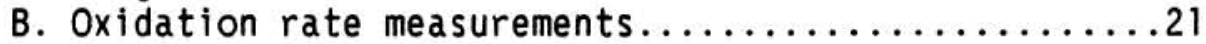

C. Sediment Dynamics..........................

3. ${ }^{13} \mathrm{C} /{ }^{12} \mathrm{C}$ ratio in the sediment column................24

A. Isotopic signature of SOC .....................

B. Isotopic signature of $\mathrm{CaCO}_{3} \ldots \ldots \ldots \ldots \ldots \ldots \ldots \ldots \ldots \ldots$

CHAPTER TWO SAMPLING AND ANALYTICAL METHODS $\ldots \ldots \ldots \ldots \ldots \ldots \ldots \ldots \ldots$

1. Site description...................................

2. Sampling strategies.................................

A. Pore water chemistry samples.....................

B. Incubation Studies.......................... 40

C. Carbon source studies.......................

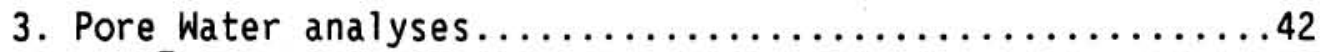

A. $\left\{\mathrm{CO}_{2}, \mathrm{Alk}, \mathrm{H}_{2} \mathrm{~S}, \mathrm{PO}_{4}, \mathrm{DOC} \ldots \ldots \ldots \ldots \ldots \ldots \ldots \ldots \ldots \ldots \ldots \ldots \ldots\right.$

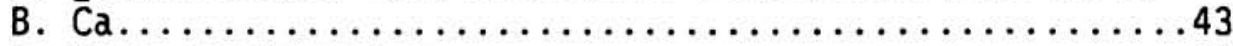

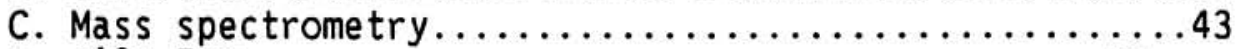

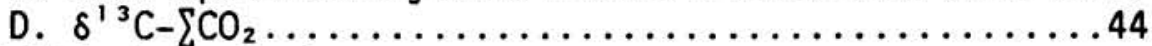

4. Solid-phase analyses.......................... 48

A. Organic carbon $\ldots \ldots \ldots \ldots \ldots \ldots \ldots \ldots \ldots \ldots \ldots \ldots \ldots, \ldots \ldots \ldots$

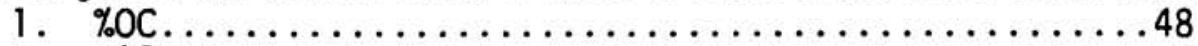

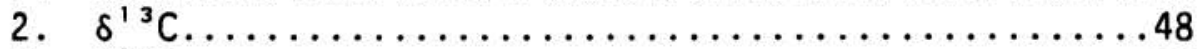

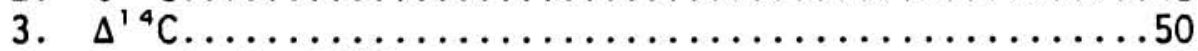

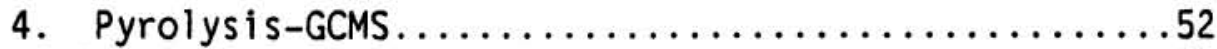

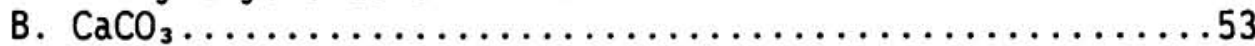

CHAPTER THREE A QUANTITATIVE ESTIMATE OF THE ANNUAL

REMINERALIZATION OF ORGANIC CARBON IN BUZZARDS

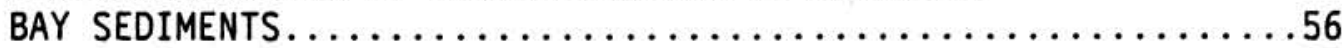

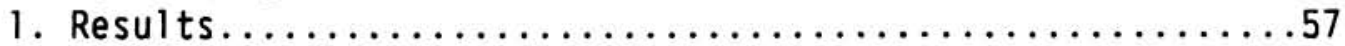

A. Solid-phase data............................... 57

B. Pore water data...........................62

2. Carbon Budget for Buzzards Bay Sediments.............70

A. Burial rate of organic carbon.................... 70

B. Oxidation rate of organic carbon.................. 70

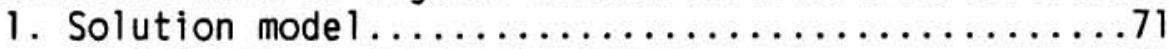

Oxidation of organic carbon................

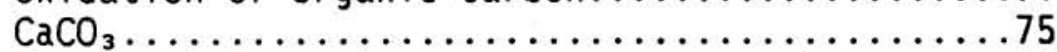

Transport Parameters......................78

Mathematical model....................... 80 
2. Solution model results..............88

3. Summary.......................... 99

4. Solid-phase model.................101

5. Solid-phase model results............107

C. Carbon Budget.....................116

CHAPTER FOUR

CHAPTER FIVE

CHAPTER SIX
THE NATURE OF THE ORGANIC MATTER UNDERGOING OXIDATION AND OTHER FACTORS AFFECTING THE

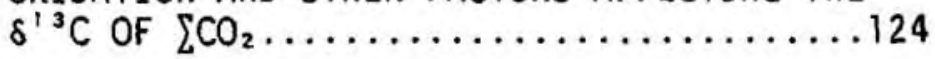

1. Introduction............................. 124

2. Results.............................. 125

A. Field Study........................ 125

B. Incubation Studies................... 132

1. Comparison of IEI and IE2 ......... 140

3. Modelling the Field Data................... 148

A. Diagenetic Model..................... 148

4. Fractionated Oxidation of SOC..............155

A. Incubation Studies................. 155

5. Diffusion of Bottom Water Carbonate Ion.......161

6. Exchange...............................67

7. June 84 Core.............................

8. Summary and Conclusions.................. 170

THE SOURCES OF ORGANIC CARBON TO THE SEDIMENTS OF BUZZARDS BAY............................ 175

1. Introduction............................... 175

2. $\delta^{1{ }^{3}} \mathrm{C}$ and $\Delta^{14} \mathrm{C}$ Results...............176

3. Pyrolysis-GCMS Results....................179

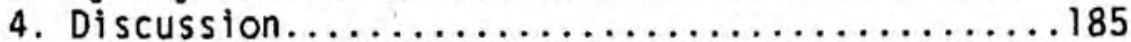

A. Surface Sediment $\Delta^{14} \mathrm{C}$ values..........185

B. Relative Contribution of Sources.........187

C. Organic Geochemical Evidence.............190

SUMMARY AND CONCLUSIONS....................

1. Remineralization of Organic Carbon...........196

2. Sources of Carbon to Pore Water $\left[\mathrm{CO}_{2} \ldots \ldots \ldots 200\right.$

3. Nature of Organic Carbon Oxidized............203

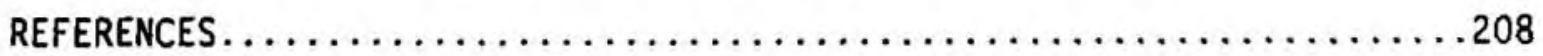

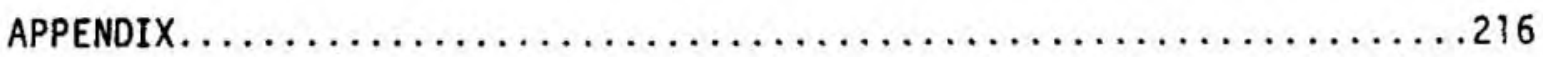

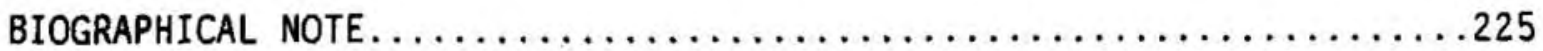




\section{LIST OF TABLES}

Table I.I

Table II.I

Table II.2

Table II. 3

Table II. 4

Table II. 5

Table III.I

Table III.2

Table III.3

Table III.4

Table III. 5

Table III. 6

Table III. 7

Table IV. 1

Table IV. 2

Table IV. 3

Table IV. 4

Table IV. 5

Table IV. 6
Stoichiometry of oxidation

Core collection dates. .39

$\delta^{13} \mathrm{C}-\sum \mathrm{CO}_{2}$ standards

CHN duplicates.

$\delta^{13} \mathrm{C}-\mathrm{SOC}$ replicates................

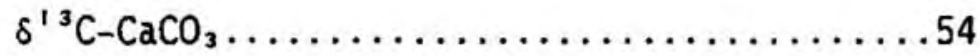

Sediment diffusion coefficients ........... 81

Solution model fitting parameters ..........93

Comparison of $J_{\text {mod }}$ and $J_{s w} \ldots \ldots \ldots \ldots \ldots$

Model-calculated values of $R_{c} \ldots \ldots \ldots \ldots \ldots$

Solid-phase model fitting parameters.........110

Model-calculated values of $k_{1}$ and $k_{2} \ldots \ldots 114$

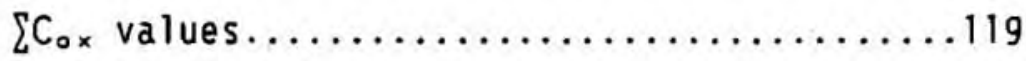

Best-fit 1 ines, $\delta^{13} \mathrm{C}$ vs. $\left[\mathrm{CO}_{2} \ldots \ldots \ldots \ldots 131\right.$

Calculated values of $R_{c}$, IEl and IE2

Ca saturation index, IEl and IE2 ......... 144

Dissolved $\mathrm{Ca}$, IEl and IE2 ............. 145

$\Delta A 1 k / \Delta \sum \mathrm{CO}_{2}$, IEl and IE2 $\ldots \ldots \ldots \ldots \ldots \ldots 6$

Calculated values of $\delta^{13} C_{a d d}$, IEl .......157 
Table IV.7

Table IV.8

Table IV.9

Table V.1

Table V.2

Table V.3
Calculated values of $\delta^{13} C_{a d d}$, IE2 ........158

$\mathrm{BW}$ and pore water $\left[\mathrm{CO}_{3}^{2-}\right]$ and $\mathrm{pH} \ldots \ldots \ldots \ldots, 163$

Calculated values of $J_{b w} \ldots \ldots \ldots \ldots \ldots \ldots 7$

Measured Values of $\delta^{13} \mathrm{C}$ and $\Delta^{14} \mathrm{C} \ldots \ldots \ldots 177$

Literature values of $\delta^{13} \mathrm{C}$ and $\Delta^{14} \mathrm{C} \ldots \ldots .178$

Relative source contributions. 


\section{LIST OF FIGURES}

Figure I.I

Figure I.2

Figure II.I

Figure II.2

Figure III.1

Figure III.2

Figure III.3

Figure III.4

Figure III.5

Figure III.6

Figure III.7

Figure III.8

Figure III.9

Figure III. 10

Figure III.1I

Figure IV.1

Figure IV.2

Figure IV.3

Figure IV.4

Figure IV.5

Figure IV. 6
Processes affecting $\delta^{13} \mathrm{C}-\left[\mathrm{CO}_{2} \ldots \ldots \ldots \ldots 16\right.$

Sources of carbon to coastal sediments..........28

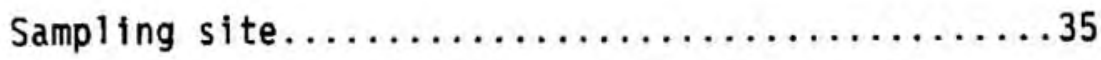

$\delta^{13} \mathrm{C}-\sum \mathrm{CO}_{2}$ stripping 1 ine...............

SOC and $\delta^{13} \mathrm{C}-\mathrm{SOC} \ldots \ldots \ldots \ldots \ldots \ldots \ldots \ldots$

Pore water profiles......................63

Solution model........................

ICP for $\mathrm{CaCO}_{3}$ vs. depth..................

Approach to steady-state.................... 84

Further steady-state approaches............... 86

Solution model fits...................... 89

Solid-phase model....................... 102

Model fit to ${ }^{210} \mathrm{~Pb}$ data................... 108

Model fit to metabolizable organic carbon........111

Temperature, $R_{c}, k_{1}$, and $\sum C_{o x}$ vs. time...121

$\delta^{13} \mathrm{C}-\sum \mathrm{CO}_{2}$ vs. $\left[\mathrm{CO}_{2}\right.$, each month......126

$\delta^{13} \mathrm{C}-\left[\mathrm{CO}_{2}\right.$ vs. $\left[\mathrm{CO}_{2}\right.$, all data........129

Pore water results for Incubation Experiments....133

Isotope model fits...................... 150

Diffusion model......................... 164

Carbonate speciation vs. pH..................171 
Figure V.1

Figure V.2

Figure V.3
Profile of $\Delta^{14} \mathrm{C}$ with depth.............. 180

Pyrolysis-GC chromatograms................. 182

Structures of vanillic acid and acetovanillon...193 


\section{ACKNOWLEDGEMENTS}

This thesis represents an enormous achievement for me, but it would never have been possible without the help. support, and encouragement of many people. I am indebted to my thesis supervisors, Ellen Druffel and Cindy Lee, who have provided friendship and support throughout my student career - from scientific guidance and financial support to hemming my skirt. The members of my committee have been very helpful. Fred Sayles let me use equipment in his $1 \mathrm{ab}$ and his knowledge of carbonate chemistry and comments on my manuscript greatly improved my thesis. Craig Taylor's expertise was invaluable in designing the incubation experiments (particularly IE2). Ed Boyle and Werner Deuser were helpful in getting me started on my project.

The laboratory work of this thesis was carried out in many labs, and the people in all those labs have been patient and very helpful. In particular, the support of Brenda Olson, Debbie Burns, Sheila Griffin, Charlie Olson, Lary Ball, Wayne Dickinson, Hovey Clifford, Eben Franks, LLoyd Keigwin, and Stu Wakeham is appreciated. These people and others have taught me methods, built equipment, sampled in all weather, and helped me in numerous other ways. Sheila Griffin and Cathy Cetta did most of the graphics. Kevin Druffel-Rodriguez helped me on one memorable sampling trip.

Perhaps the hardest thing for me to do during this thesis was to maintain my sanity. The chemistry students at MIT and WHOI and the friends I've made over the years have helped make this possible. For all the cheers, tears, and fears, I thank Pat, Leslie, Bruce, Brenda, Charlie, Art, John, Ellen, and Cindy. The softball crew and all the women with whom I've played basketball and soccer have provide lots of entertainment and have been important in teaching me self-confidence.

I'd like to thank my grandmother for bulliding a house in Prince Edward Island. I acquired my love of and respect for the ocean during my summers there. My family has always supported me in my academic endeavors, and I thank them for this. Bill has been a tremendous supporter of the work I've done; if it weren't for him, I would have left this program a long time ago. On a practical level, he taught me how to minimize the agonies of working in a glove bag along with many other lab secrets and encouraged my dabblings in math. He solved the time-dependent differential equation I used in Chapter III.

Finally, I'd like to thank all who helped me through a particularly rough time, whether they knew they did or not.

Financial support was provided by the Education Office of the Massachusetts Institute of Technology/Woods Hole Oceanographic Institution Joint Program in Oceanography, by an Andrew W. Mellon Foundation grant to the Coastal Research Center, WHOI, and by the National Science Foundation under grant NSF OCE83-15412. 


\section{Chapter I}

\section{INTRODUCTION}

\section{I.1. Introduction}

A portion of the organic matter produced in surface waters of the ocean falls to the sediments where it can be used by the benthic community. In nearshore environments, this community is abundant and active. A fraction of the organic matter reaching the sediments is remineralized to $\mathrm{CO}_{2}$. Quantitative estimates of the rates of decomposition of organic carbon to $\mathrm{CO}_{2}$ are essential to understanding global carbon cycling through time and to assess the effect of man on the carbon cycle. Coastal areas are likely to reflect the effect of the increased anthropogenic activities of the past century best. Due to mixing and burial processes, substances have only a brief residence time in the zone of biological activity, and the relationship between the rates at which organisms can use organic carbon and its rate of transport through the sediment column will determine how much carbon is preserved.

The overall oxidation of sedimentary organic carbon (SOC) is summarized in the following equation: 


$$
\begin{array}{r}
\left(\mathrm{CH}_{2} \mathrm{O}\right)_{x}\left(\mathrm{NH}_{3}\right)_{4}\left(\mathrm{H}_{3} \mathrm{PO}_{4}\right)_{2}+\mathrm{EA} \longrightarrow \\
\text { reduced } \left.\mathrm{EA}+x \mathrm{CO}_{2}+y \mathrm{HNO}_{3} \text { (or } \mathrm{NH}_{3}\right)+ \\
2 \mathrm{H}_{3} \mathrm{PO}_{4}+\mathrm{bH}_{2} \mathrm{O} \\
\text { Where } \mathrm{EA}=\text { electron acceptor }
\end{array}
$$

The electron acceptors used in the reaction vary systematically in the order: $\mathrm{O}_{2}, \mathrm{NO}_{3}$ and $\mathrm{Mn}, \mathrm{Fe}, \mathrm{SO}_{4}$, and $\mathrm{CO}_{2}$ and small organic compounds (Froelich et al., 1979). The rate of remineralization is related to the quantity and quality of the organic matter available to the microbial community (Westrich and Berner, 1984).

The oxidation of organic carbon in sediments can be traced through changes in the $\delta^{13} \mathrm{C}^{*}$ of dissolved inorganic carbon $\left(\sum \mathrm{CO}_{2}\right)$ in pore water. Unfractionated oxidation of SOC adds carbon that is isotopically light to the dissolved pool of carbon. The $\delta^{13} \mathrm{C}$ of marine organic carbon $(-20.0 \mathrm{o} / 00)$ is significantly different from that of $\mathrm{CaCO}_{3}(+1.0 \mathrm{O} / 00)$ and bottom water $\mathrm{CCO}_{2}(0.0 \mathrm{o} / \infty)$. Previous studies of seawater and sediments have shown that a decrease in the $\delta^{13} \mathrm{C}$ of dissolved inorganic carbon can be attributed to the oxidation of organic carbon. Deuser (1970) used the relationship between $\delta^{13} \mathrm{C}$ and $\mathrm{SCO}_{2}$ in the deep sea to constrain a sulfur budget for the Black Sea. Kroopnik (1985) derived a linear equation relating the isotopic profile of $\mathrm{SCO}_{2}$ and apparent oxygen utilization after demonstrating that the $\delta^{13} \mathrm{C}$ of the deep water was controlled mainly by the oxidation of organic carbon in the water column. They made the

\footnotetext{
${ }^{\star} \delta^{13} \mathrm{C}=\left(R_{S} / R_{P O B}-1\right) 10^{3} \quad$ where $R_{s, P O B}={ }^{13} \mathrm{C} /{ }^{12} \mathrm{C}$ ratio in sample and PDB respectively
} 
assumptions that the organic matter had Redfield stoichiometry and a constant isotopic signature.

Presley and Kaplan (1968) investigated two cores from basins off Southern California. In both cores the $\delta^{13} \mathrm{C}$ of the pore water $\sum \mathrm{CO}_{2}$ decreased to a value approaching that of marine organic matter while the concentration of $\mathrm{SCO}_{2}$ was increasing. Miller (1980) has documented decreases in the $\delta^{13} \mathrm{C}-\sum \mathrm{CO}_{2}$ in pore waters from sediments of the eastern equatorial Atlantic. Gradients in $\delta^{13} \mathrm{C}-\sum \mathrm{CO}_{2}$ of up to $-1.0 \mathrm{o} / 00 / \mathrm{cm}$ were observed in surface pore waters from the central equatorial Pacific (McCorkle et a1.,1985). These decreases are attributable to the oxidation of organic carbon in the sediments.

In a study of the sediments of Saanich Inlet, BC (Nissenbaum, et al, 1972; Brown, et al, 1972), it was found that the $\delta^{13} \mathrm{C}$ of $\mathrm{SCO}_{2}$ increased from -11.2 to 9.4 o/oo over a depth interval of $190 \mathrm{~cm}$ while the $\sum \mathrm{CO}_{2}$ concentration increased from 2.7 to $36.2 \mathrm{mmol} / 1$. This was contrary to what would be expected from the input of $\mathrm{CO}_{2}$ formed from the unfractionated oxidation of organic matter. The presence of $\mathrm{CH}_{4}$ in the interstitial waters led Nissenbaum et a1. (1972) to propose that the enrichment was due to the preferential reduction of ${ }^{12} \mathrm{CO}_{2}$ by $\mathrm{CH}_{4}$-forming bacteria. This process would leave the remaining $\left[\mathrm{CO}_{2}\right.$ enriched in ${ }^{13} \mathrm{C}$. The fermentation of organic acids may also have contributed to the enrichment as both processes lead to ${ }^{13} \mathrm{C}$-depleted $\mathrm{CH}_{4}$ and ${ }^{13} \mathrm{C}$-enriched $\mathrm{CO}_{2}$ (Rosenfeld and Silverman, 1959; Deines, 1980). A study investigating the process of methanogenesis has confirmed that the production of $\mathrm{CH}_{4}$ is coincident with an increase in $\delta^{13} \mathrm{C}-\left[\mathrm{CO}_{2}\right.$ (Alperin and Reeburgh, 1984). 
It is evident that the $\delta^{13} \mathrm{C}$ of $\left[\mathrm{CO}_{2}\right.$ in pore waters is altered by the oxidation of organic matter. Figure I-l summarizes the major processes that affect this profile. This study presents the stable carbon isotope ratios $\left(\delta^{13} \mathrm{C}\right)$ of organic and inorganic carbon in coastal sediments and pore waters collected on a seasonal basis. It provides a detailed study of the decomposition of organic carbon in sediments from Buzzards Bay, MA, a coastal marine environment. The goals of this research are 1) to quantify the amount of organic carbon remineralized, 2) to study the nature of the organic carbon oxidized, and 3 ) to investigate the sources of organic carbon to these nearshore sediments.

The following sections will review work on the remineralization of organic carbon and the ${ }^{13} \mathrm{C} /{ }^{12} \mathrm{C}$ ratio in sediments.

I.2. Remineralization of organic carbon in sediments

The remineralization of organic carbon in sediments greatly alters the pore water and solid-phase sediment chemistry. This remineralization and its effects have been studied in a variety of environments - from very anoxic muds in Cape Lookout Bight (Martens and Klump, 1984) to sediments underlying oligotrophic gyres in the central Pacific (e.g. Grundmanis and Murray, 1982). Many studies have relied on modelling the concentrations of the various electron acceptors used to oxidize organic carbon to draw conclusions about the rates of biogeochemical processes which occur during the early diagenesis of sediments. Other groups of researchers have concentrated on using measured rates of the oxidation of organic carbon to understand sedimentary 
Figure I.1. The major processes that can affect the $\delta^{13} \mathrm{C}$ of $\mathrm{SCO}_{2}$ in pore waters of coastal sediments. 
Figure I.1

bottom water $\mathrm{SCO}_{2}$
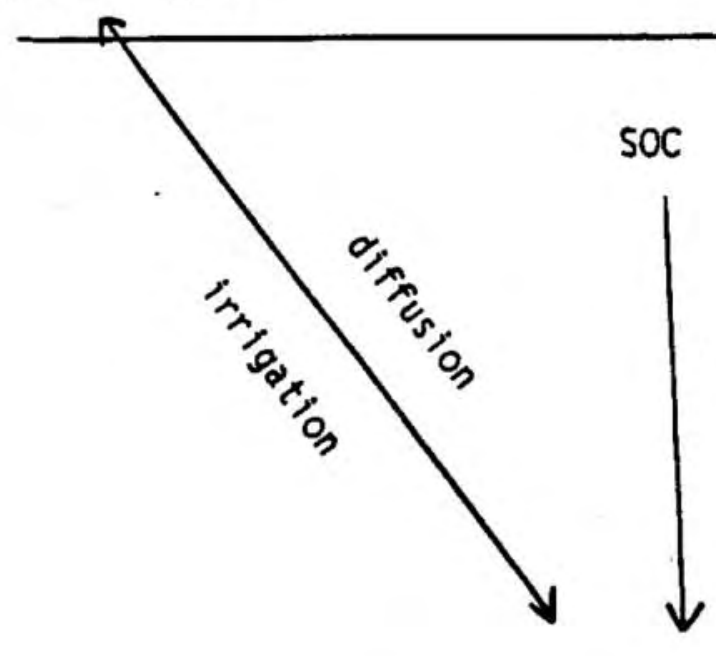

$\sum \mathrm{CO}_{2}$

oxidation

sediment water interface
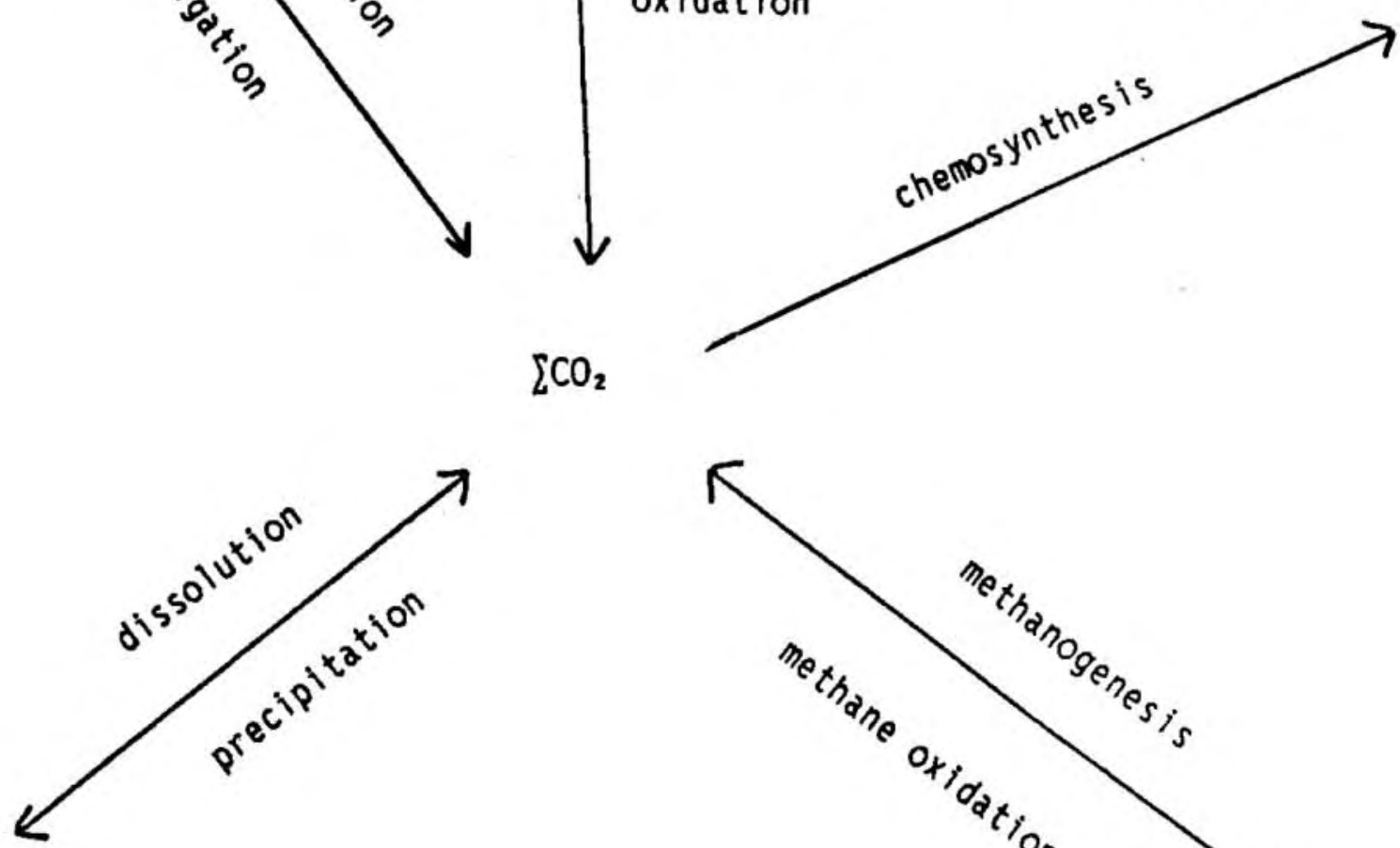

$\mathrm{CaCO}_{3}$

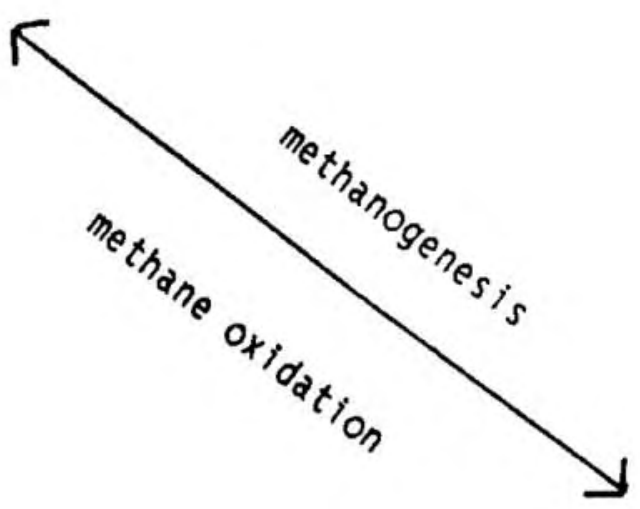

Me thane 
diagenesis. These studies have been hampered by the inability to measure $\mathrm{O}_{2}$ on a fine enough scale, until recently; uncertainties as to the relative proportions of chemical vs. biological oxidation; and inconsistencies between radiolsotope determined rates and model calculated rates of oxidation of SOC. Recently, there has been a trend toward coordinated investigations of sediments to alleviate confusion.

\section{I.2.A. Diagenetic models}

Most diagenetic modelling has concentrated on relating changes in the profiles of electron acceptor concentrations to the remineralization of organic matter. Deep ocean studies have used profiles of dissolved inorganic nitrogen (DIN) to constrain the rates of organic matter degradation. Jahnke et al. (1982) modelled the profiles of DIN to quantitatively evaluate the importance of oxygen and nitrogen as oxidants in oceanic sediments. They calculated that denitrification never oxidized more than $30 \%$ of the organic matter oxidized by $\mathrm{O}_{2}$. Solid phase and pore water analyses were used to investigate organic matter remineralization in sediments of the equatorial Pacific (Grundmanis and Murray, 1982). The stoichiometry of the organic matter undergoing degradation was calculated from the solid-phase and pore water data. The solid-phase data yielded a $\mathrm{C} / \mathrm{N}$ ratio of 13.1 , while the pore water data gave a ratio of 8.6. Both these estimates deviate from that of organic matter with Redfield stoichiometry (6.6).

Stoichiometric models have been applied to alkalinity $(A \mid k), \sum \mathrm{CO}_{2}$, and dissolved Ca data from deep-sea sediments (Emerson, 1980; Sayles, 1981). These models assume that organic matter with Redfield stoichiometry $(C: N: P=$ 106:16:1) is oxidized to $\mathrm{CO}_{2}$ in the manner shown in Table I-1. Changes in 


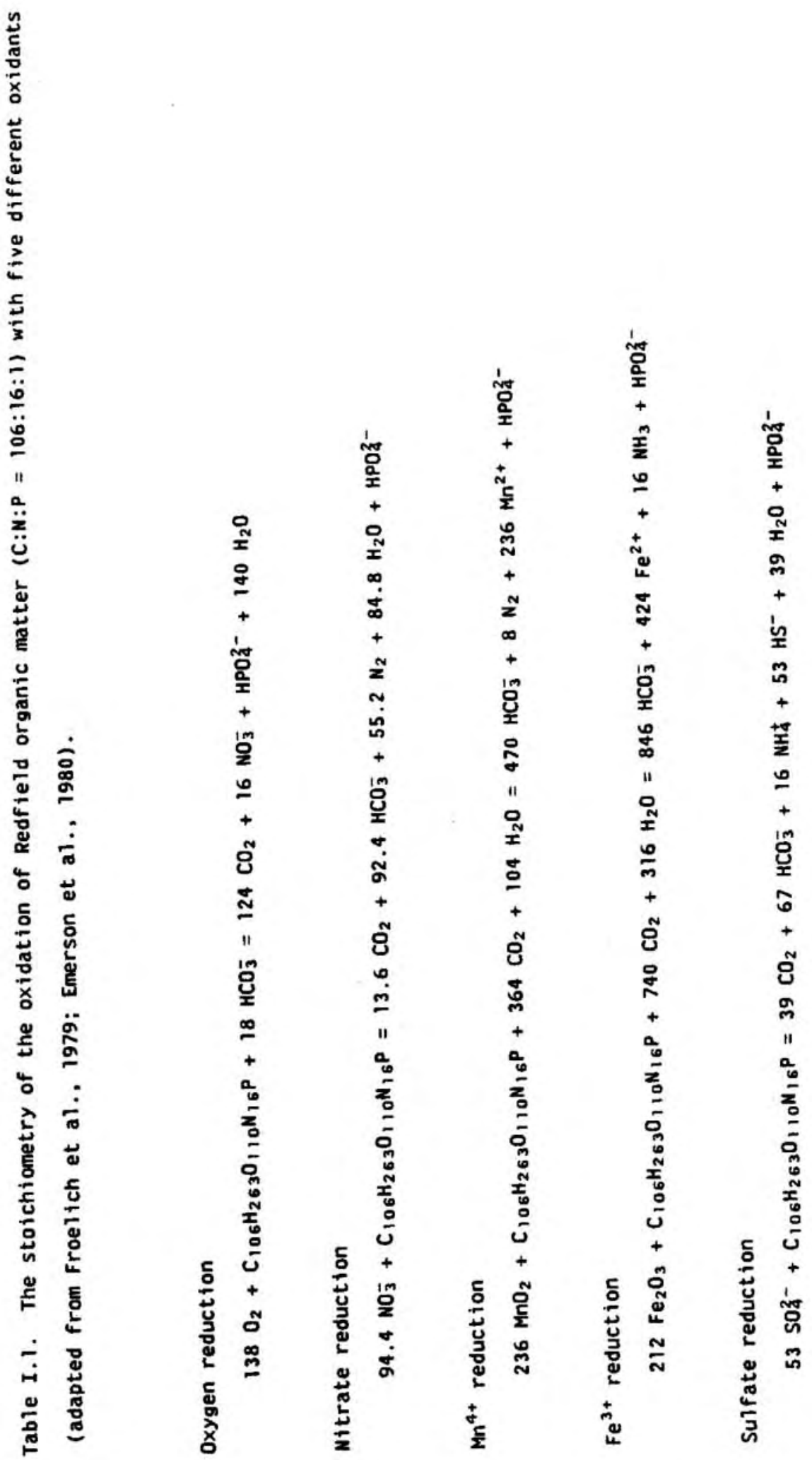


Alk and $\sum \mathrm{CO}_{2}$ with depth are predicted considering equilibrium with $\mathrm{CaCO}_{3}$, the stoichiometry of oxidation, the diffusion of solutes through pore waters, and assumed rates of oxidation of organic carbon. This approach has been useful in understanding the carbonate system in deep sea sediments; however its applicability to nearshore systems is more complex. Deep sea sediments are deposited in a relatively quiescent environment where solute transport can be described by molecular diffusion. In contrast, the active benthic community in nearshore sediments can enhance solute transport through mixing and irrigation. Increased productivity and a shorter water column lead to a greater supply of highly labile organic matter to the sediment-water interface. The full suite of reactions listed in Table I-1 can occur over a distance as small as $0.5 \mathrm{~cm}$.

In nearshore sediments, oxygen and sulfate are the most important electron acceptors with respect to oxidizing capacity. Sulfate is by far the most important electron acceptor in coastal sediments; it can account for up to $70 \%$ of the total sediment respiration (Howarth, 1984). Oxygen reduction is limited by its ability to diffuse into the sediments. The depth of the diffusive sublayer is a function of the turbulence of the water near the sediment water interface (Jorgensen and Revsbech, 1985). An additional source of $\mathrm{O}_{2}$ to the pore waters arises from the introduction of bottom water at depth in sediments from the irrigating action of benthic organisms. This has been shown with the use of $\mathrm{O}_{2}$ microelectrodes in sediments (Jorgensen and Revsbech, 1985). Modelling of $\mathrm{O}_{2}$ and $\mathrm{SO}_{4}$ profiles to calculate organic carbon oxidation rates has been hampered by an incomplete knowledge of the mixing regime in nearshore sediments. 


\section{I.2.B. Oxidation rate measurements}

The rate of oxidation of organic carbon in sediments has been calculated from two different types of incubation experiments. In jar experiments, the disappearance of an oxidant over time is measured. These experiments usually occur over a period of weeks or months. Stable and radio- isotope tracer experiments are used to measure oxidation rates in a matter of minutes to hours. Labelled oxidant is introduced to the sediment and its disappearance over time, as well as the production of labelled intermediates and end products is monitored. Net rates are measured in jar experiments, while gross rates are measured in tracer experiments (Reeburgh, 1983).

Many incubation studies have been performed on coastal sediments where $\mathrm{SO}_{4}$ is the most important oxidant. Jar experiments (Aller and Yingst, 1980), as well as ${ }^{35} \mathrm{SO}_{4}$ experiments (Westrich, 1985) have been performend on sediments from Long Island Sound. Jorgensen (1977) has used ${ }^{35} \mathrm{SO}_{4}$ to measure sulfate reduction rates in a Danish fjord. The ranges in sulfate reduction rates measured in surface sediments at locations affected by irrigation in the above studies are 0.32 to $3.2 \times 10^{-9} \mathrm{~mol} / \mathrm{L}-\mathrm{yr}$. Rates in surface sediments are always much greater than those in deeper sediments, and rates in warm months are greater than in cold months.

The major disadvantage of all incubation studies is the necessity of removing the sediment from its natural environment. In sampling for an incubation study, the sediments are handled carefully to avoid disrupting the existing microbial community. Although an incubation study will always artificially close an open system, the comparison of model-predicted with measured oxidation rates can provide an important check on the validity of 
each method.

\section{I.2.C. Sediment dynamics}

Modelling in nearshore sediments is complicated by an active bioturbated zone, uncertainty as to the validity of steady state models, and difficulty in assessing the flux of material to the sediment-water interface. The discussions of diagenetic models and oxidation rate measurements have emphasized that coastal sediments are deposited in a dynamic environment. The consequences of activity of the macrobenthos must be addressed when discussing the remineralization of organic carbon in nearshore sediments. Solid-phase mixing can introduce labile organic matter to depths it would never reach by sedimentation alone. During the oxidation of organic matter, a solid is converted to dissolved species. The mechanisms and rates of transport of particulate material in the sediments are usually very different from those of dissolved material; particle mixing rate coefficients can be as much as 4 orders of magnitude less than solute diffusion coefficients. Mixing coefficients ranging from $10^{-6}$ to $10^{-8} \mathrm{~cm}^{2} / \mathrm{sec}$ have been measured for solids in Long Island Sound (Benninger et al., 1979). Modelling of solute profiles in Long Island Sound sediments yielded diffusion coefficients around $10^{-6} \mathrm{~cm}^{2} / \mathrm{sec}$ (Goldhaber et al., 1977). Vanderborght et al. (1977) calculated "enhanced" diffusion coefficients on the order of $10^{-4} \mathrm{~cm}^{2} / \mathrm{sec}$ from profiles of dissolved silica in sediments from the North Sea. In a nearshore environment enhanced mixing and transport of solid and dissolved species arises because of wave and current stirring and the activities of burrowing organisms such as bivalves and polychaete worms.

Irrigation of the burrows constructed by benthic animals radically alters 
the solute distributions encountered in sediment pore waters (Aller, 1980). The effects of irrigation on pore water profiles in coastal sediments have been treated in different ways: with an "enhanced" diffusion coefficient, and with two non-local source models. Initially, irrigation was modelled with an "enhanced" diffusion coefficient (McCaffrey et al., 1980). Aller (1980) and Emerson et al. (1984) have presented non-local source models; these models assume the presence of burrow and tube structures alters the average geometry of molecular diffusion. Vertical advection-diffusion is no longer considered a good approximation of the real environment; the burrows create a cylindrical diffusion geometry in the sediment immediately surrounding them. The removal of the decomposition products of organic matter remineralization is enhanced in the burrow system. Products generated "deep" in the sediment, i.e. away from the sediment-water interface, need only diffuse through the burrow wall to the animal's tube where they will be flushed out by irrigation. Thus an increased macrofaunal community lowers the pore water concentration of metabolites through irrigation. Results from these models mimic measured profiles more accurately than models using "enhanced" diffusion coefficients. Aller's model is more complex; a drawback to applying this model is the number of parameters related to the local fauna that must be defined - average burrow spacing, average burrow radius, and average burrow length.

In the model of Emerson et al. (1984), irrigation is considered to be a mode of transport that is capable of exchanging overlying water with points in the sediment that are removed from the sediment-water interface. The transport of dissolved metabolites from pore waters deep in the sediment is accomplished by "flushing" burrows in this sediment with bottom water. 
Solutes which have accumulated in the burrows by diffusion from the surrounding sediments are removed. Irrigation has introduced bottom water to a point in the sediments it could never have reached without the existence of burrows. They define a non-local source or sink term such that:

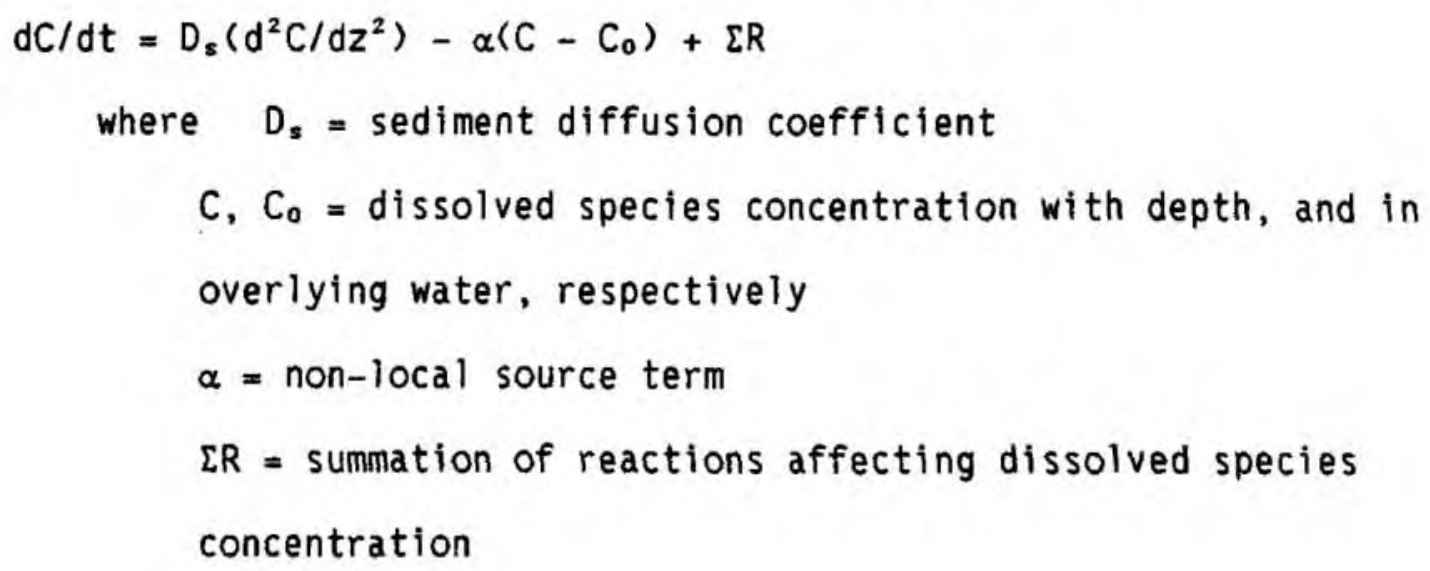

This model has been shown mathematically to approximate Aller's burrow model (Boudreau, 1984). The main problem that arises in using non-local exchange models is that the chemistry of exchange across a burrow lining cannot be considered. Aller (1983) has shown that each solute can react differently in crossing a burrow lining, and that different burrow linings can affect the transport of a particular solute across the lining differently.

\section{I.3. THE ${ }^{\prime 3} \underline{\mathrm{C}} /{ }^{\prime 2} \mathrm{C}$ RATIO IN THE SEDIMENT COLUMN}

The $\delta^{13} \mathrm{C}$ of $\left\{\mathrm{CO}_{2}\right.$ in pore waters is most sensitive to the remineralization of organic carbon and to the dissolution or precipitation of $\mathrm{CaCO}_{3}$ in the sediment column. An understanding of the factors affecting the $\delta^{13} \mathrm{C}$ of $\mathrm{SOC}$ and $\mathrm{CaCO}_{3}$ is essential to understanding the $\delta^{13} \mathrm{C}$ of 
$\sum \mathrm{CO}_{2}$. These factors are discussed below.

\section{I.3.A. Isotopic signature of $\mathrm{SOC}$}

Investigations of SOC in sediment cores have attributed isotopic shifts with depth in the core to microbial activity, temporal changes in the isotopic signal of the source organic matter, and selective remineralization of different fractions of the organic matter (e.g. Peterson et al., 1980; Sackett and Thompson, 1963; Sackett, 1964). It is probable that some combination of all these factors is responsible for the observed changes. In a recent review article, Deines (1980) discussed the possible effects of diagenesis on the isotopic signature of SOC. He concluded that the evidence for early alteration is insufficient and that the observed differences of $\delta^{13} \mathrm{C}$ with depth in sediment are due to changes in the source due to past changes in climate, circulation, or productivity. The studies he used to reach this conclusion span a wide range of marine environments, from salt marsh to open ocean. However, reexamination of the studies coupled with more recent data may suggest that observable fractionations do occur during early diagenesis, particularly in coastal areas where the supply of fresh organic matter to the sediments is high.

A study of sediment from Baffin Bay, Texas, (Behrens and Frishman, 1971), showed an increase in $\delta^{13} \mathrm{C}$ of SOC with depth which was attributed to the preferential remineralization of ${ }^{12} \mathrm{C}$. Johnson and Calder (1973) measured the $\delta^{13} \mathrm{C}$ of SOC in two cores from a salt marsh where the organic carbon content of the sediments decreased from 3.5 to $0.1 \%$ by weight. In a slightly reducing core the $\delta^{13} \mathrm{C}$ decreased from -16.9 to -19.4 o/ o over $45 \mathrm{~cm}$, while in a strongly oxidizing core the $\delta^{13} \mathrm{C}$ increased from -23.9 to -20.9 
o/co over $37 \mathrm{~cm}$. The authors of this paper were interested in the isotopic difference between sites and did not try to explain the depth-related change. In a study of the sediments of the Pettaquamscutt River, an anoxic basin in Rhode Island, two major shifts were seen in the $\delta^{13} \mathrm{C}$ of SOC (Hites et al., 1980). It was unclear whether these were related to relatively minor changes in the percent organic carbon in the core, to diagenetic alteration, or to temperature or salinity differences that would alter the $\delta^{13} \mathrm{C}$ of the input POC. These studies do not provide clear evidence that the $\delta^{13} \mathrm{C}$ of SOC is altered during early diagenesis, but they do suggest that systematic studies of sediment systems may indicate that fractionation is occurring.

The microbial activity responsible for the oxidation of organic carbon to $\mathrm{CO}_{2}$ could result in an alteration of the $\delta^{13} \mathrm{C}$ of the residual SOC.

Physical chemical experiments have shown that the ${ }^{12} \mathrm{C}-{ }^{12} \mathrm{C}$ bond is weaker than the ${ }^{13} \mathrm{C}-{ }^{12} \mathrm{C}$ bond. It would be energetically and kinetically favorable for an organism to cleave the weaker bond. In a study of the oxidation of lactate by a sulfate reducing organism, Kaplan and Rittenberg (1964) found that the $\mathrm{CO}_{2}$ given off as well as the carbon incorporated during growth were enriched in ${ }^{12} \mathrm{C}$ with respect to the initial lactate. In this system, both the oxidation of lactate and the assimilation of carbon were occurring with fractionation. This fractionation resulted in the $\mathrm{CO}_{2}$ given off and the organic carbon produced differing isotopically from the starting material. It is uncertain whether fractionation is universal microbiologically or to what extent it occurs in the natural environment. Chemosynthetic activity in sediments is also responsible for changes in the $\delta^{13} \mathrm{C}$ of SOC. Microorganisms can use reduced inorganic sulfur compounds to 
fix $\mathrm{CO}_{2}$ from pore waters (Kuenen and Beudeker, 1982), forming organic carbon that is highly depleted in ${ }^{13} \mathrm{C}$, e.g. $\delta^{13} \mathrm{C}=-35$ o/oo (Peterson et al. 1980). The importance of chemosynthesis in coastal sediments is unknown, although in irrigated coastal sediments it can occur at oniy $5 \%$ of the respiration rate (Howarth, 1984). In summary, microbial fractionation appears possible, even likely, but its net effect (+ or - ) on the $\delta^{13} \mathrm{C}$ of $\mathrm{CCO}_{2}$ is not known.

Temporal changes in the POC deposited at the sediment water interface may also complicate the isotopic profile of SOC. Figure I-2 lists some of the sources of organic carbon to a coastal environment like Buzzards Bay and their isotopic signature. Three isotopically distinct sources can be identified terrestrial $(-25$ to $-27 \circ / 00)$, planktonic $(-18$ to $-220 / 00)$, and salt marsh $(-10$ to $-160 / 00)$ carbon. The relative supply of these sources of organic carbon may vary seasonally and over historical time. Seasonal fluctuations in temperature and salinity can have an effect on the signature of plankton (Deuser et a1., 1968; Degens et al., 1968). Sediment mixing in a coastal area usually homogenizes the surface sediments enough to eliminate seasonal differences in the $\delta^{13} \mathrm{C}$ of sedimentary POC. However, hurricanes might deposit such large amounts of material with an isotopic signature significantly different from the normal input, that the signal would not be lost by sediment mixing. On a longer time scale, the organic carbon deposited now is probably more depleted in ${ }^{13} \mathrm{C}$ than that deposited prior to the Industrial Revolution (Hites et al., 1980).

Another point to consider when analyzing the isotopic signature of SOC involves the selective remineralization of certain fractions of the organic 
Figure I.2. The major sources of organic carbon to the sediments in a coastal environment and their isotopic signature. Although Zostera marina (eel grass) grows in the marine system, it is included with salt marsh carbon because it is a vascular plant. 


\section{Figure I.2}

Terrestrial Organic Carbon

Natural $\quad \delta^{13} \mathrm{C}=-260 / 00$

Anthropogenic

Fossil fuel, fresh or burned $\quad \delta^{13} \mathrm{C}=-22$ to $-26 \% / 00$

Sewage sludge $\quad \delta^{13} \mathrm{C}=-260 / 00$

Salt Marsh Organic Carbon

Spartina alterniflora $\quad \delta^{13} \mathrm{C}=-13$ to -16 o/oo

zostera $\quad \delta^{\prime 3} \mathrm{C}=-110 / 00$

Marine Organic Carbon

plankton $\quad \delta^{13} \mathrm{C}=-18$ to $-220 / 00$

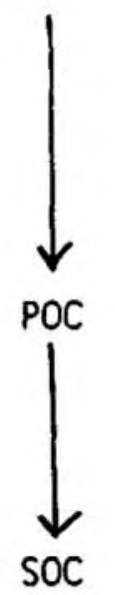


matter. In a coastal environment the different sources of organic carbon to the sediments also have different reactivities. Radiocarbon studies of Long Island Sound sediments have indicated that the residence time of easily metabolizable organic carbon is on the order of 2 years (Benoit et al., 1979; Turekian et al., 1980; Berner, 1980b). In their study, planktonic carbon $\left(\delta^{13} \mathrm{C}=-200 / 00, \Delta^{14} \mathrm{C}=1220 / 00\right)$ was defined as the easily metabolizable fraction, and terrestrial carbon $\left(\delta^{13} \mathrm{C}=-260 / 00, \Delta^{14} \mathrm{C}\right.$ $=-242$ o/00) plus a small percentage of the planktonic carbon were considered refractory. This distinction was made to facilitate the interpretation of the ${ }^{14} \mathrm{C}$ profile. The refractory material will pass through the well-mixed layer and remain as a permanent record in the sediment column. The signature of $\left[\mathrm{CO}_{2}\right.$ will be affected by this differential remineralization. Unfractionated oxidation of planktonic carbon will add $\mathrm{CO}_{2}$ with a $\delta^{13} \mathrm{C}$ of -20 o/oo, while oxidation of the refractory material will add $\mathrm{CO}_{2}$ with a $\delta^{13} \mathrm{C}$ of $-26 \% / 00$.

The selective remineralization of individual compound classes may affect the $\delta^{13} \mathrm{C}$ of SOC as well as that of $\left[\mathrm{CO}_{2}\right.$ because the $\delta^{13} \mathrm{C}$ of individual compounds are different from that of bulk SOC (Degens, 1969). Pectins are enriched in ${ }^{13} \mathrm{C}$ by up to 5 o/00 (i.e. $\delta^{13} \mathrm{C}=-150 / 00$ ), while lipids are depleted by as much as $90 / 00\left(\delta^{13} \mathrm{C}=-290 / 00\right)$ (Degens, 1969). Amino acids have been found to be enriched in ${ }^{13} \mathrm{C}\left(8^{13} \mathrm{C}=-12\right.$ to $-190 / 00$ ) relative to bulk plant material because the carboxyl carbon of the amino acid is isotopically heavy (Abelson and Hoering, 1961); the magnitude of the enrichment differs for each amino acid. Certain classes of organic compounds are more labile than others and are metabolized more rapidly. For 
example, in Saanich Inlet, fatty acid and chlorin concentrations normalized to organic carbon decrease with depth, while the normalized hydrocarbon concentration remains essentially constant (Brown et al. 1972). The $\delta^{13} \mathrm{C}$ of hydrocarbons, free and hydrolyzable fatty acids, hydrolyzable amino acids, and humic acids were analyzed in this study. The fatty acids and hydrocarbons were found to be depleted in ${ }^{13} \mathrm{C}$ relative to bulk SOC, while the amino acids, humics, and fulvics had a $\delta^{13} \mathrm{C}$ similar to that of bulk SOC. The more rapid remineralization of fatty acids in the surface sediments would add $\mathrm{CO}_{2}$ to the $\left[\mathrm{CO}_{2}\right.$ pool that is isotopically lighter than the bulk organic carbon. In Mangrove Lake, Bermuda, a decrease in $\delta^{13} \mathrm{C}$ of the total organic matter with depth was related to the diagenetic loss of carbohydrates, which are relatively enriched in ${ }^{13} \mathrm{C}$ (Spiker and Hatcher, 1984). This would have the opposite effect on the $\delta^{13} \mathrm{C}$ of $\left[\mathrm{CO}_{2}\right.$ as the oxidation of fatty acids.

It is evident that there are numerous factors affecting the $\delta^{13} \mathrm{C}$ of SOC in coastal sediments. The $\delta{ }^{13} \mathrm{C}$ of $\sum \mathrm{CO}_{2}$ generated during the oxidation of SOC will be influenced according to the relative importance of each factor.

\section{I.3.8. Isotopic Signature of $\mathrm{CaCO}_{3}$}

The isotopic signature of dissolved inorganic carbon will also be influenced by the dissolution or precipitation of calcium carbonate. In oxidized sediments, there are two major processes that produce the acids that dissolve $\mathrm{CaCO}_{3}$. The oxidation of organic matter with $\mathrm{O}_{2}$ produces the weak acid $\mathrm{CO}_{2}$, and the oxidation of solid-phase sulfides with $\mathrm{O}_{2}$ can generate a large amount of strong acid $\left(\mathrm{H}_{2} \mathrm{SO}_{4}\right)$. The production of acid and, thus, 
the extent of dissolution in nearshore sediments is a function of physical disturbance and blological reworking. Rapid mixing in surface sediments maintains a zone with oxygenated pore waters. Deeper benthic mixing is responsible for transporting solid-phase sulfides to the oxic zone. Aller (1982) has observed that the dissolution of $1-10 \mathrm{mg} \mathrm{CaCO} / \mathrm{cm}^{2}-\mathrm{yr}$ occurs near the sediment water interface in Long Island Sound. Precipitation occurs in reducing sediments. In the sulfate reducing zone, alkalinity increases with each mole of sulfate reduced. The increase in alkalinity can create a supersaturation with respect to aragonite and calcite and precipitation may result. Sayles (1981) has shown that, while precipitation occurs in reducing sediments in the Atlantic, these sediments also remain supersaturated with respect to calcite.

In order to understand the $\delta^{13} \mathrm{C}$ of $\mathrm{CCO}_{2}$, the amount of $\mathrm{CaCO}_{3}$ dissolution or precipitation occurring in the sediments must be quantified, and the $\delta^{13} \mathrm{C}$ of the $\mathrm{CO}_{3}^{2-}$ added or removed must be known. Sayles (1981) has used the profile of Ca concentration to quantify the loss or gain of $\mathrm{CaCO}_{3}$. Dissolution occurs with little or no fractionation, but precipitation is not as simple a process. The equilibrium fractionation between bicarbonate and aragonite and between bicarbonate and caicite during precipitation has been quantified by Rubinson and Clayton (1969). However, the rate of precipitation must be considered when calculating the isotopic fractionation accompanying $\mathrm{CaCO}_{3}$ precipitation. Turner (1982) has shown that kinetic fractionation accompanies quick precipitation, but that slow precipitation results in $\mathrm{CaCO}_{3}$ that is in isotopic equilibrium with bicarbonate. Modelling the isotopic effect of precipitation in sediments is 
complicated further by changes in the $\delta^{13} \mathrm{C}$ of $\mathrm{CCO}_{2}$ with depth in the core. The isotope ratio of $\left[\mathrm{CO}_{2}\right.$, and thus $\mathrm{CO}_{3}^{2-}$, will be changing down core due to the addition of ${ }^{13} \mathrm{C}$-depleted $\mathrm{CO}_{2}$ from the oxidation of organic matter. 


\section{SAMPLING AND ANALYTICAL METHODS}

Understanding the cycling of carbon in Buzzards Bay sediments required the measurement of a large number of different species in both the solid and pore water phases. In this chapter I will describe the sampling site and discuss the sampling strategies and the analytical methods I used to measure pore-water and solid-phase components.

\section{II.1. Site Description}

The research described here was conducted at a site in Buzzards Bay, MA (41 $31.25 \mathrm{~N}, 7045.7 \mathrm{~W}$; Figure II.1). Buzzards Bay is on the western boundary of Cape Cod, is $28 \mathrm{mi}$ long, $8 \mathrm{mi}$ wide, and has an average depth of $15 \mathrm{~m}$. The maximum elevation of the watershed is $200-300 \mathrm{ft}$ and the area is poorly drained (Hough, 1940) as there are no major rivers emptying into the bay. The sediments range from coarse sands to silty clays; the coarsest sediments are closest to the edges of the bay.

The site used in this study is the same as that used by Martin (1985). The relationship of the site to other study sites in Buzzards Bay is detailed in Martin (1985). The sampling site is in $15 \mathrm{~m}$ of water and is predominantly silty-clay sediments. The sediments contain approximately $20 \mathrm{mg} / \mathrm{gdw}$ organic 
Figure II.1. The location of the sampling site (marked with an ' $x$ ') and Station $P$ in Buzzards Bay, Massachusetts. 
Figure II.I

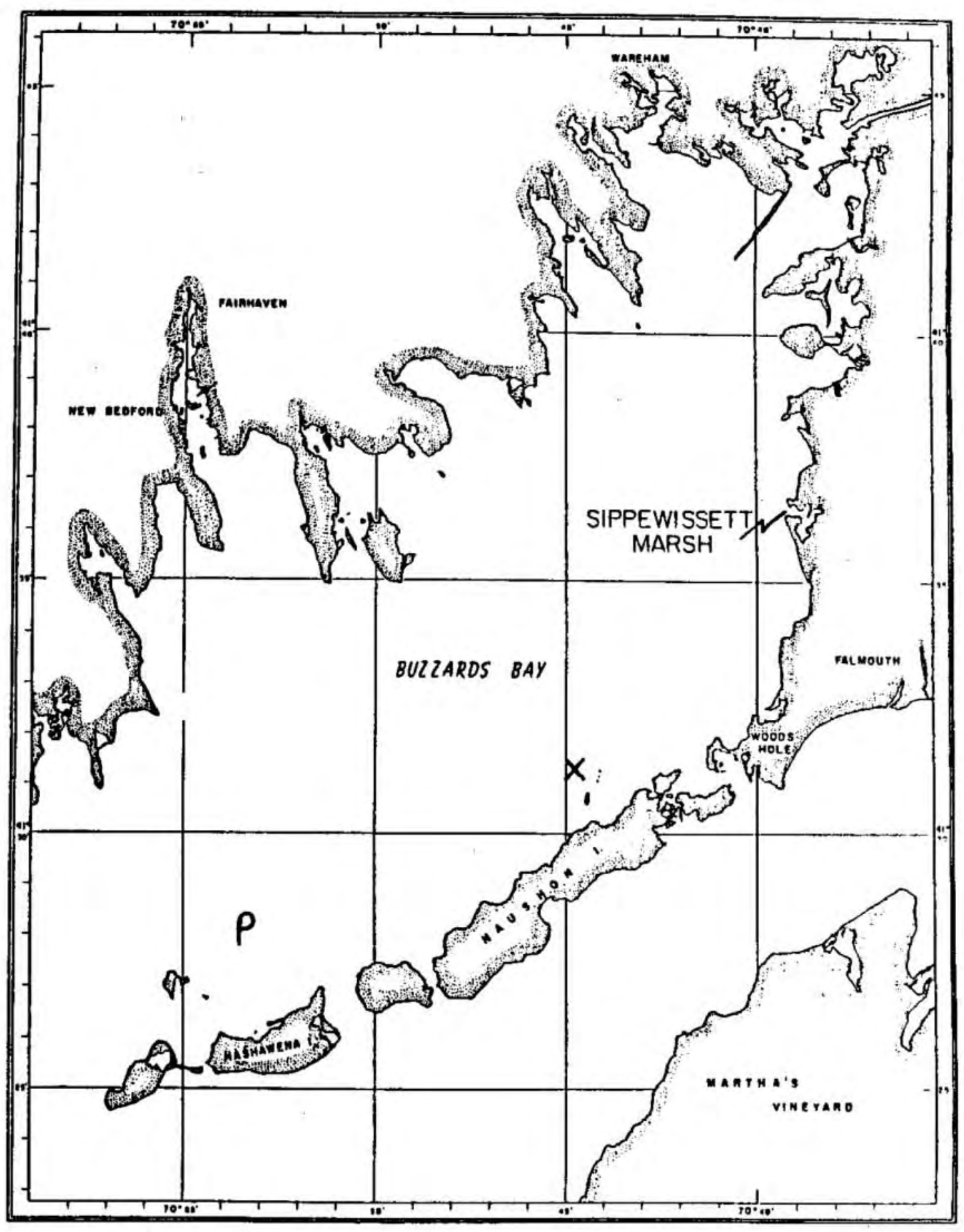


carbon and are primarily sulfate-reducing. The top $30 \mathrm{~cm}$ of the sediment column were studied. At this site, the $\mathrm{SO}_{4}$ concentration at $30 \mathrm{~cm}$ was never depleted more than $10-15 \%$ of the bottom water value. The faunal assemblage at this site is a Nephthys incisa-Nucula proxima community (Sanders, 1958). This community is comprised mostly of deposit feeders, organisms that find their food on or in the sediment (Sanders, 1960).

A study by Rhoads (1967) indicated that extensive biogenic reworking of the sediments occurred to depths of $2-3 \mathrm{~cm}$. This observation was substantiated by ${ }^{234}$ Th studies (Martin, 1985); the maximum depth of penetration of ${ }^{234} \mathrm{Th}$ was $3.0 \mathrm{~cm}$. Transport studies of the solution-phase using ${ }^{222} \mathrm{Rn}$ (Martin, 1985) indicated that there was irrigation of the sediments in warm months occurring to depths of at least $20 \mathrm{~cm}$.

Several geochemical studies have taken place at Station P (Figure II.1). This station is similar to the site used here; the sediments are silty clays and are approximately $20-30 \mathrm{mg} \mathrm{C} / \mathrm{gdw}$ (Henrichs, 1980). Studies at this station have demonstrated that certain fractions of the organic matter are decomposed more rapidly than others; fatty acids and amino acids are transformed before sterols (Farrington et a1., 1977; Lee, et al., 1977; Henrichs, 1980). Remineralization rates calculated from pore water and sediment profiles differed by a factor of about 4 (Henrichs, 1980). This was attributed to a differential transport of solids and solutes and to more rapid remineralization occurring in the oxic zone.

II.2. Sampling strategies

II.2.A. Pore water chemistry samples

The pore water chemistry of six cores was analyzed in this study. The 
dates of collection and water temperature at time of collection are listed in Table II.1. All cores were collected by SCUBA divers. In October 83 an $8 \mathrm{~cm}$ diameter PVC core liner was used to collect the core and after that all cores were collected with a $20 \mathrm{~cm}$ diameter PVC core liner. The tops of the core liners were sealed under water and the cores were sealed tightly on deck and rapidily transported to the laboratory where they were sectioned in a $\mathrm{N}_{2}$-filled glove bag. Sectioning was aiways completed within 12-15 hours of core collection.

In the glove bag, mud was transferred to $50 \mathrm{ml}$ centrifuge tubes which were sealed, removed from the glove bag, and centrifuged at 3000-3500 rpm for 15-20 min to isolate the pore water. No attempt was made to maintain the in-situ temperature during either sectioning or centrifuging. This should not have made a large difference for the cores collected in summer and fall, however there may be some temperature artifacts in the samples collected in the cold months. Plastic centrifuge tubes were used until March 85 after which glass (Corex) tubes were used. After spinning down, the centrifuge tubes were returned to a glove bag, opened, and pore water was drawn into $10 \mathrm{ml}$ syringes. Both glass and plastic syringes were used; the use of glass syringes will be noted in the tables. All pore water was passed through a $0.45 \mu \mathrm{m}$ Nuclepore polycarbonate filter. The first aliquot of pore water was discarded and the next was filtered directly into a $1 \mathrm{ml}$ syringe and analyzed within the hour for $\left[\mathrm{CO}_{2}\right.$. In October $83, \sum \mathrm{CO}_{2}$ was not measured; $\mathrm{pH}$ was measured on the first alliquot of pore water. The electrode was in the glove bag and $\mathrm{pH}$ was measured immediately after collecting the sample. The next aliquot of water was filtered into a $3 \mathrm{ml}$ syringe, transferred quickly 
Table II.1. Core collection dates and bottom water temperature at the time of collection.

$\begin{array}{ll} & \begin{array}{l}\text { Bottom } \\ \text { Water }\end{array} \\ \text { Core date } & \underline{\text { Temp. }{ }^{\circ} \mathrm{C}} \\ \text { Oct. 17, 83 } & 15 \\ \text { Dec. 16,83 } & 7 \\ \text { June 20,84 } & 16 \\ \text { Aug. 30,84 } & 21 \\ \text { March 26, 85 } & 4 \\ \text { Oct. 30,85 } & 14\end{array}$


into a $2 \mathrm{ml}$ poisoned septum vial, and sealed with no headspace. Again, both glass and plastic syringes were used at this stage; the use of glass will be noted in the tables. A few crystals of mecuric chloride were used as poison. This sample was stored in the dark at $4^{\circ} \mathrm{C}$ until analysed for $\delta^{13} \mathrm{C}-\left[\mathrm{CO}_{2}\right.$. In March 85, a different method of collecting the sample for $\left[\mathrm{CO}_{2}\right.$ analysis was used. After collection of the sample for $\delta^{13} \mathrm{C}-\sum \mathrm{CO}_{2}$, a $1-\mathrm{ml}$ aliquot of the filtered sample was stored in a poisoned $\left(\mathrm{HgCl}_{2}\right) 2-\mathrm{ml}$ septum vial. The samples were analyzed within 10 hours of collection. Subsequent aliquots were collected for sulfide ( $1 \mathrm{ml})$, phosphate $(1 \mathrm{ml})$, alkalinity $(1 \mathrm{ml}), \operatorname{DOC}(1-2 \mathrm{ml})$, calcium $(1.5-3 \mathrm{ml})$, and sulfate $(0.5-1 \mathrm{~m} 1)$ analyses. Mud for solid phase analyses was transferred to clean glass jars and frozen at $-40^{\circ} \mathrm{C}$. Mud was stored at this temperature until freeze-drying.

\section{II.2.B. Incubation studies-1 and 2}

Incubation Experiment 1 (IE1). In early March 85, a Soutar box core with a surface area of $0.04 \mathrm{~m}^{2}$ was used to collect mud for the first incubation study. Four depth intervals were sampled $-0-2 \mathrm{~cm}, 9-11 \mathrm{~cm}, 17-20 \mathrm{~cm}$, and 22-25 cm. Each interval was transferred to a Nalgene beaker and quickly removed to a $\mathrm{N}_{2}-$ filled glove bag. The sediment was homogenized by stirring and packed into 50-ml Corex centrifuge tubes. The centrifuge tubes were kept in the dark at $15^{\circ} \mathrm{C}$ and sampled at different times by centrifuging and collecting the pore water; handling and analysis of the samples from the incubation study was the same as that of the pore-water chemistry samples. The method used for the collection and analysis of $\mathrm{COO}_{2}$ samples for the 
March 85 field core was used. The porosity of the surface sediment was so great that over $24 \mathrm{hr}$ some separation of pore water from the solid-phase occurred. To counteract this the tubes were inverted daily.

Incubation Experiment 2 (IE2). In August, 1985, divers inserted 4 core liners as close to each other as possible, capped them, and returned them to the ship. The core liners had been prepared with pre-cut holes that were sealed with duct tape. Small incubation tubes (approximately $9 \mathrm{~cm}$ long) were cut from $30 \mathrm{~mm} O D$ glass tubing, and each tube was fitted with a plunger from a 50-ml disposable syringe. To sample the sediment for this incubation study, one of the pre-cut holes was uncovered and a small piston sub-core was taken with the glass tubing. The ends of the glass tubing were sealed immediately with black rubber stoppers. Sediment from 3 depths $(10-13,12-15$, and 18-21 cm) was collected this way. This procedure did not work for the surface samples. To sample the surface sediment, the caps were removed from the core liners, the overlying water was drained off, and the incubation tube was filled from the top of the core.

The filled tubes were protected from light and maintained at room temperature until sampling; handling and analysis of the samples was the same as that of the pore-water chemistry samples.

\section{II.2.C. Carbon source studies}

Mud from the cores collected for pore water studies was frozen until analysis for $\delta^{13} \mathrm{C}-\mathrm{SOC}$ and pyrolysis/GCMS. Samples representative of possible sources of organic matter to Buzzards Bay were collected locally. Standing spartina alterniflora was collected at Great Sippewissett Marsh 
(GSM), and eel grass was collected from a creek connecting Little Sippewissett Marsh to Buzzards Bay. A sample of flocculent matter at the bottom of the creek draining GSM was collected by disturbing the sediment, collecting water, and filtering the water. A soll sample from near the shores of the Weweantic River in Wareham was collected and frozen until analysis.

A gravity core designed by $\mathrm{J}$. Broda was used to obtain a core at the study site for the analysis of natural levels of radiocarbon in Buzzards Bay sediments. A 1-meter long core was collected in a 10-cm diameter PVC core liner. The core was stored at $4^{\circ} \mathrm{C}$ overnight, sectioned at 5-6 cm intervals and stored frozen in glass jars until analysis.

\section{II.3. Pore water Analyses}

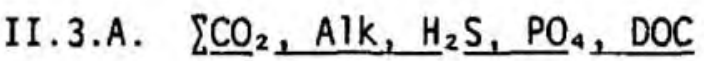

Standard analytical methods were used for these analyses. $\mathrm{ZCO}_{2}$ samples were acidified with $2 \mathrm{~N} \mathrm{H}_{3} \mathrm{PO}_{4}$, stripped with helium, and measured by gas chromatography. This analysis was made immediately after sample collection. In March, 1985 and for IEl a headspace technique was used to measure $\left[\mathrm{CO}_{2}\right.$. One $\mathrm{ml}$ of sample was drawn into a syringe and acidified with sulfuric acid. $\mathrm{CO}_{2}$-free air was added and the sample was agitated. The headspace was analyzed for $\mathrm{CO}_{2}$ by gas chromatography. Alkalinity was measured by Gran titration. Sulfide was measured by the method of Gilboa-Garber (1971) against a methylene-blue solution. The solution was calibrated against actual sulfide standards according to Fonselius (1976). Phosphate was measured colorimetrically as described in Strickland and Parsons 
(1972). These analyses were made within 1-3 days of sample collection. A wet persulfate oxidation adapted from Menzel and Vaccaro (1964) was used to analyze DOC. The $\mathrm{CO}_{2}$ collected from the oxidation was analyzed by gas chromatography.

\section{II.2.B. $\underline{\mathrm{Ca}}$}

A high precision EGTA titration described by Shiller and Gieskes (1980) was used to measure calcium. The precision of the Ca titration was $\pm 0.2 \%$; this precision was obtained only when the sample was pre-titrated to > $99 \%$ with EGTA. The procedure was standardized against IAPSO seawater, assuming a $\mathrm{Ca} / \mathrm{Cl}$ ratio of $0.02109 \mathrm{~g} \mathrm{Ca} / \mathrm{o} / 00 \mathrm{Cl}$. As it is possible that the EGTA solution used as titrant may change overnight, IAPSO standards were run at the beginning of each day. Duplicate $1.5 \mathrm{~g}$ samples were weighed precisely into small Erlenmeyer flasks. The concentration was estimated based on the concentration of the sample preceding it and on the expected trend in the data. The first sample was pre-titrated to $>90 \%$ to see if the estimate was correct. The second sample was pre-titrated to $>99 \%$ based on the value found for the first sample. When possible, a third sample was titrated.

\section{II.2.C. Mass Spectrometry}

All ${ }^{13} \mathrm{C}$ isotopic measurements were made on a VG-Micromass $602 \mathrm{E}$ mass spectrometer. At the start of each operating day a standard was run to check the integrity of the reference gas. If pore water or carbonate samples were being analysed, NBS-20 $\left(\delta^{13} C_{P O B}=-1.06 \mathrm{o} / 00\right)$ was run, and, if organic carbon samples were being analysed, NBS-2l $\left(\delta^{13} C_{P D B}=-28.100 / 00\right)$ was 
run. All isotopic values are reported relative to PDB.

\section{3.D. $\quad \delta^{13} \underline{\mathrm{C}}-\left[\underline{\mathrm{CO}}_{2}\right.$}

Poisoned samples for the analysis of $\delta^{13} \mathrm{C}-\sum \mathrm{CO}_{2}$ were stored at $4^{\circ} \mathrm{C}$ in the dark until analysis. The samples were always stripped and analyzed within 6 weeks of collection and usually within 2 weeks. A stripping line designed by $\mathrm{W}$. Curry was used to analyse the $\delta^{13} \mathrm{C}$ of pore water $\mathrm{CCO}_{2}$. A diagram of the stripping line is shown in Fig. II.2. One $\mathrm{ml}$ of $100 \%$ $\mathrm{H}_{3} \mathrm{PO}_{4}$ is introduced to the stripping chamber through the ground-glass joint on the stripping chamber. Care is taken not to get any acid on this joint. The injection port is attached to the stripping chamber and the line is evacuated. A more detailed drawing of the injection port is shown in Figure II.2. Two dry ice-isopropanol slush baths are used to trap water, and a liquid nitrogen trap is placed on a third trap to collect $\mathrm{CO}_{2}$. Approximately $1 \mathrm{ml}$ of pore water is injected into the stripping chamber from a tuberculin syringe through the septum. The septum is changed after each sample. Nitrogen gas, which had been passed through ascarite, was used to mix the acid and water and to strip $\mathrm{CO}_{2}$ from the solution. The sample was subjected to three purges with $\mathrm{N}_{2}$ at 5 minute intervals during the $15 \mathrm{~min}$ stripping period. The $\mathrm{CO}_{2}$ was isolated in the third trap, purified, and transferred to a flask for storage or introduced to the mass spec.

Local seawater, collected at Chemotaxis dock, was used to determine the reproducibility of the measurement. Data collected from the analys is of the water is listed in Table II.2. It appeared that there were occasionally "flyers" in the analyses, although there was no known reason to discard any 
Figure II.2. Diagram of the stripping line used for the analysis of $\delta^{13} \mathrm{C}-\sum \mathrm{CO}_{2}$. 
Figure II.2

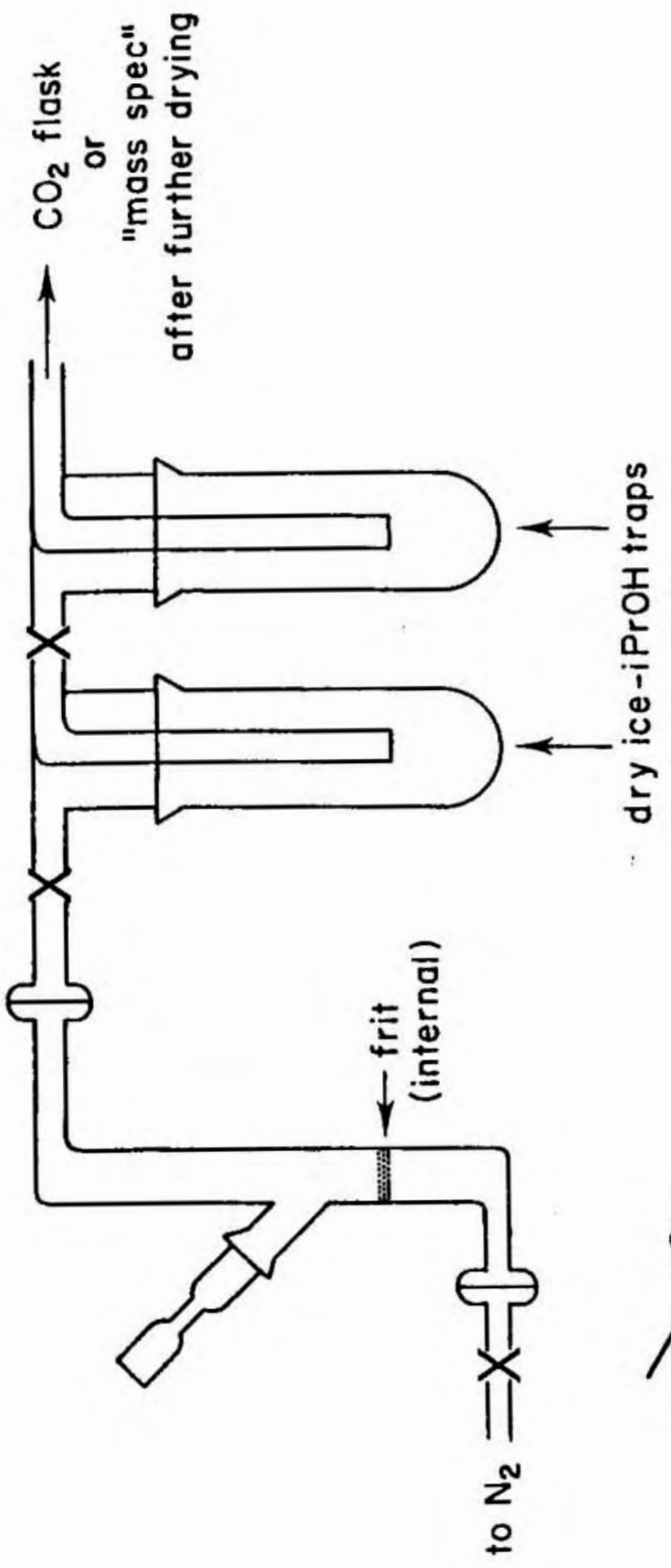

$\frac{\text { ำ }}{\text { (日) }}$

苛

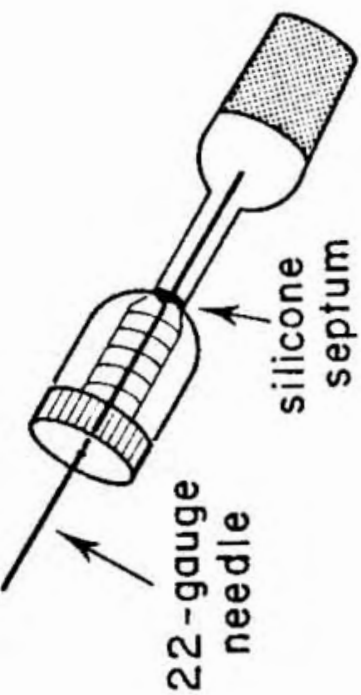


Table II.2. $\delta^{13} \mathrm{C}$ of Chemotaxis water standards. Absolute values of the different standards differ because the samples were collected on different dates.

$\begin{array}{lll}a & b & c \\ 10 / 83 & \underline{2 / 85} & \underline{5 / 85} \\ & & \\ 0.37 & 0.71 & 0.80 \\ 1.10 & 0.66 & 1.07 \\ 1.38 & 0.49 & 0.66 \\ 1.22 & 0.62 & 1.14 \\ 1.41 & 0.38 & 0.89 \\ 1.05 & 0.04 & 0.84 \\ 0.81 & & 1.18 \\ 1.38 & & 1.06 \\ 0.84 & & 1.00\end{array}$

1.50

1.12

0.44

1.23

1.36
$x=1.08$
0.48
0.96
$s=0.35$
0.25
0.17 
data points. When running the samples on the mass spectrometer, the magnitude of the ion current is proportional to the size of the sample. It was noted that the "flyers" were much smaller than the other samples, i.e. the ion current was 5-10 times lower. It appears that occasionally the stripping procedure is ineffective or the sample is lost during stripping. When the mass spectrometer ion current was used as a qualitative indicator of the stripping efficiency, the results obtained from stripping Chemotaxis water were more precise (Table II.2c). Results from samples collected after February 85 will be reported with a smaller standard deviation than before.

II.4. Solid-phase analyses

II.4.A. Organic carbon

1. $\% \mathrm{OC}$

Portions of freeze-dried sediments were placed in petri dishes, treated with $1 \mathrm{~N} \mathrm{HCl}$, and allowed to air-dry in a hood. Based on total carbon analyses from a Perkin-Elmer $\mathrm{CHN}$ analyzer, one acid treatment was enough to remove all the $\mathrm{CaCO}_{3}$. Drying was completed by placing the petri dishes in an oven at $60^{\circ} \mathrm{C}$ for 1 hour. The acid-treated samples were analyzed for CHN on a Perkin-Elmer CHN analyser by Bonnie Woodward; the precision of the analysis is reported to be $\pm 2 \%$ ( $B$. Woodward, personal communication). Duplicate analyses were run on samples from December 83 ; the results are presented in Table II.3. These analyses indicate that the precision of the sediment samples is closer to $\pm 4 \%$.

2. $\delta^{13} \underline{C}$

Samples for stable isotope measurements were prepared using a closed-tube 
Table II.3. Measured values of duplicate analyses of $\%$ C for samples from December 83. The analysis was made on a Perkin Elmer CHN analyzer. The column headed $\Delta \%$ represents the absolute value of the difference between columns $I$ and II, 1 .e. $\left|\% O C_{1}-\% O C_{1},\right|$. The average difference, $x$, corresponds to an analytical precision of $\pm 4 \%$.

$\begin{array}{lllll}\text { Date } & \text { Depth } & \text { I } & \text { II } & \Delta \% \\ 10 / 83 & 0-0.1 & 2.11 & 2.30 & 0.19 \\ 12 / 83 & 0-0.5 & 1.56 & -- & \\ & 0.5-1 & 2.87 & 2.91 & 0.04 \\ & 1-1.5 & 3.24 & 3.29 & 0.05 \\ & 1.5-2 & 3.12 & 3.11 & 0.01 \\ & 2-2.5 & 2.84 & 2.97 & 0.13 \\ & 2.5-3 & 2.94 & 2.82 & 0.12 \\ & 3-3.5 & 2.90 & 2.82 & 0.08 \\ & 3.5-4 & 2.89 & 2.57 & 0.32 \\ & 4-5 & 1.99 & 1.99 & 0.00 \\ & 6-7 & 1.84 & 1.89 & 0.05 \\ & 8-9 & 1.94 & 1.90 & 0.04 \\ & 15-16 & 1.67 & 1.74 & 0.07 \\ & 20-21 & 1.63 & 1.62 & 0.01 \\ & 25-26 & 1.68 & 1.65 & 0.03 \\ & & & & \\ & & & & x=0.07\end{array}$


combustion method first reported by Sofer (1980). Combustion tubes were made from $8 \mathrm{~mm}$ OD standard-wall Pyrex tubing and were pre-combusted at $500^{\circ} \mathrm{C}$ for at least 3 hours. CuO used in the combustion was pre-combusted at $900^{\circ} \mathrm{C}$ for at least 1 hour. Blanks run at the start of my research indicated that contamination from reactants was negligible. Approximately $100 \mathrm{mg}$ of acid-treated sediment was transferred to a combustion tube; approximately $2 \mathrm{mg}$ of CuO was added to the tube. After drawing a narrow neck, the tubes were evacuated to a pressure of less than $2 \mu \mathrm{m}$ and sealed under vacuum. The samples were combusted at $500^{\circ} \mathrm{C}$ for 1 hour. Standards and certain samples were combusted at $900^{\circ} \mathrm{C}$ using quartz combustion tubes. The procedure was the same as that for Pyrex combustion tubes except that approximately $2 \mathrm{mg} \mathrm{Cu}$ was added to the tubes in addition to CuO. The samples were combusted at $900^{\circ} \mathrm{C}$ for 1 hour. Samples combusted at $900^{\circ} \mathrm{C}$ will be designated with a q.t. in tables. Combusted samples were opened under vacuum and the $\mathrm{CO}_{2}$ generated from the combustion was purified and collected for isotopic analysis.

One freeze-dried sediment sample was analyzed separately 9 times. The measured $\delta^{13} \mathrm{C}^{\prime} \mathrm{s}$ are reported in Table II.4. A few samples were combusted at both high and low temperatures and no significant difference was observed in the measured $\delta^{13} \mathrm{C}$. This is consistent with the results reported in Fry et al. (1984).

\section{3. $\Delta^{14} \underline{\mathrm{C}}$}

Natural radiocarbon levels were measured in organic carbon samples according to procedures outlined in Griffin and Druffel (1985). Sediment samples were acidified, rinsed with distilled water, and dried prior to burning. Spartina and eel grass samples were dried and crushed prior to 
Table II.4. $\delta^{13} \mathrm{C}$ of solid-phase organic carbon measured on sediment from 22-24 cm collected in October 83 and separated into 9 samples.

$$
\begin{aligned}
& \delta^{13} C_{P O B} \\
& -20.5 \\
& -20.6 \\
& -20.5 \\
& -20.5 \\
& -20.5 \\
& -20.6 \\
& -20.4 \\
& -20.6 \\
& -20.5
\end{aligned}
$$

$$
x=-20.5 \pm 0.10 / 00
$$


burning. In short, the combustion is performed at high temperature in a large Vycor tube ( $31 \mathrm{~mm} 00,24 \mathrm{~mm}$ ID) with a flow of oxygen for a period of $1-4$ hours. The samples were counted as $\mathrm{CO}_{2}$ in a $200 \mathrm{cc}$ copper gas proportional beta counter. An error of $\pm 80 / 00$ was obtained for these samples. Larger samples ( $1-2 \mathrm{~g} \mathrm{C})$ were converted to $\mathrm{C}_{2} \mathrm{H}_{2}$ via a 1 ithium carbide intermediate and counted for three 2-day periods in two quartz gas proportional beta counters. An error of $\pm 30 / 00$ was obtained for these samples. Radiocarbon measurements are reported as $\Delta^{14} C$, defined as the per mil $(0 / 00)$ deviation from the activity of nineteenth century wood, taken as $95 \%$ of the activity of the NBS oxalic acid standard. Each measurement was corrected for decay from the time of formation until A.D. 1950 and for isotope fractionation by normalising to a $\delta^{13} C_{P O B}=-250 / 00$ (Broecker and 01son, 1961):

$$
\Delta^{14} \mathrm{C}=\delta^{14} \mathrm{C}-2\left(\delta^{13} \mathrm{C}+25\right)\left(1+\delta^{14} \mathrm{C} / 1000\right)
$$

The radiocarbon age is reported as years B.P. (before present) using the Libby half-life for ${ }^{14} \mathrm{C}$ of 5568 years.

\section{Pyrolysis-GCMS}

Pyrolysis-GCMS was performed on selected organic carbon samples as part of the organic carbon source study. Details of the analysis are described in Whelan et al. (1986). Briefly, a dried, acidified sample ( $10 \mathrm{mg})$ was introduced to a desorption probe and heated from 250 to $550^{\circ} \mathrm{C}$. The compounds formed during pyrolysis are split, One fraction is passed through a GC, and another is collected and later run on the GC-mass spectrometer (Carlo Erba model $4160 \mathrm{GC}$ equipped with a cryogenic oven attachment connected to a Finnigan 4500 quadrupole mass spectrometer). 


\section{II.4.8. $\mathrm{CaCO}_{3}$}

\section{1. $\delta^{13} \underline{C}$}

A variety of methods were used to measure the $\delta^{13} \mathrm{C}$ of $\mathrm{CaCO}_{3}$ in the sediments. Problems arose because of the very low concentration of $\mathrm{CaCO}_{3}$ in the sediments $(<0.2 \%$ by weight) and because of the presence of acid-volatile sulfides in the sediments. The $\delta^{13} \mathrm{C}$ of individual shells and shell fragments in the sediments was measured, and attempts were made to measure the $\delta^{13} \mathrm{C}$ of bulk, or "amorphous", $\mathrm{CaCO}_{3}$ in the sediments.

Shells and shell fragments were picked from the dried sediments, ground to a powder, baked under vacuum at $370^{\circ} \mathrm{C}$ for 1 hour, reacted to $\mathrm{CO}_{2}$ in $100 \%$ $\mathrm{H}_{3} \mathrm{PO}_{4}$, and analysed on the mass spectrometer.

It is unclear whether the methods I used to determine the $\left[\mathrm{CO}_{2}\right.$ of bulk $\mathrm{CaCO}_{3}$ in the sediments were successful. Freeze-drying the sediments left carbon from the pore water $\mathrm{CCO}_{2}$ in the solid sample. In an effort to remove this carbon the sediments were soaked overnight in distilled water, filtered, rinsed a few times with distilled water, and dried in an oven at $60^{\circ} \mathrm{C}$. Rinsed sediments were acidified with $5 \% \mathrm{H}_{3} \mathrm{PO}_{4}$ in evacuated reaction flasks at $25^{\circ} \mathrm{C}$ overnight. Dilute phosphoric acid was used to prevent oxidation of organic matter with acid (Miller, 1980). Aiso, rinsed sediments were combusted in a muffle furnace at $450^{\circ} \mathrm{C}$ for at least 6 hours to remove most of the organic matter and then acidified with $10 \% \mathrm{H}_{3} \mathrm{PO}_{4}$ at $25^{\circ} \mathrm{C}$ overnight. The isotope results are listed in Table II.5.

The $\delta^{13} \mathrm{C}$ of the $\mathrm{CO}_{2}$ collected from acidifying bulk rinsed sediment is very different from that of the shells collected from the sediment. Large shell fragments were removed from the bulk sediment before it was acidified, 
Table II.5. Measured values of $\delta^{13} \mathrm{C}_{\mathrm{POB}}$ of $\mathrm{CaCO}_{3}$. The date listed refers to the core from which the shells or sediment were taken. The depth listed $(z$, in $\mathrm{cm})$ refers to the horizon from which the shells or sediment were taken.

\section{Shells and fragments}

from unrinsed sediments

$\begin{array}{lll}\underline{z} & \text { date } & \delta^{13} \underline{\mathrm{C}} \\ 6-7 & 6 / 84 & 2.79,3.04 \\ 8-9 & 6 / 84 & 1.79 \\ 22-23 & 6 / 84 & 2.00,2.09 \\ 25-26 & 6 / 84 & 1.43,1.43\end{array}$

from rinsed sediments

$8-9 \quad 6 / 84 \quad 1.15,1.11$

$22-23 \quad 6 / 84 \quad 0.96,0.78$

$22-24 \quad 10 / 83 \quad 1.50,1.56$

Bulk rinsed sediment

$\begin{array}{lll}0-0.5 & 6 / 84 & -3.81\end{array}$

$\begin{array}{lll}2-2.5 & 6 / 84 & -2.72\end{array}$

22-24 10/83 $\quad-1.47$ 
and it is possible that there are two isotopically distinct pools of $\mathrm{CaCO}_{3}$ in the sediments - shell fragments and amorphous $\mathrm{CaCO}_{3}$. However, the amount of $\mathrm{CO}_{2}$ generated upon acidification of the sediments appeared to be very large considering the amount of $\mathrm{CaCO}_{3}$ measured in the sediments. An absolute measurement of the $\mathrm{CO}_{2}$ generated was not made. More detailed analyses of the sediment must be made before the bulk sediment numbers measured here can be fully understood. The values collected from the shell fragments will be used in the following chapters as the $\delta^{13} \mathrm{C}_{\text {of }} \mathrm{CaCO}_{3}$. If the actual value is significantly lower, the conclusions reached in Chapter IV will be strengthened. 


\section{A QUANTITATIVE ESTIMATE OF THE ANNUAL REMINERALIZATION OF ORGANIC CARBON IN BUZZARDS BAY SEDIMENTS}

The total amount of organic carbon remineralized annually at the sediment water interface and in the sediment column at a site in Buzzards Bay, MA can be estimated from sediment and pore water data collected seasonally. The Buzzards Bay site contains silty-clay sediments and is representative of silty-clay sediments frequently found in coastal waters. In this chapter, a carbon budget will be constructed using estimates of the amount of carbon remineralized to $\mathrm{CO}_{2}$ in the sediments plus the amount preserved in the sediments. The amount of carbon oxidized plus that preserved in the sediments is representative of the total amount of carbon reaching the sediment water interface. To construct a carbon budget, it is necessary to know the amount of carbon in the sediments, the rate at which it is oxidized, and the rate at which it is buried. The amount of carbon in the sediments can be measured directly. The rate of burial of organic carbon can be calculated from its concentration and the rate of sedimentation. The rate of oxidation of organic matter can be estimated from mathematical models fitted to solution and solid-phase data obtained seasonally. Results from these models must be weighted appropriately to calculate an annual average remineralization of 
organic carbon. The sampling and analytical methods used were described in Chapter II.

\section{I Results}

Solid-phase and pore water data will be presented in this section. Six cores were collected at different times of the year over a period of two years to investigate seasonality in the oxidation of organic carbon. The solid-phase data are presented in Figure III.l a to e and are listed in Table AI. 1 of Appendix I. The pore water data are presented in Figure III.2a to $f$ and are listed in Table AI.1 of Appendix I.

\section{III.1.A. Solid-phase data}

Profiles of organic carbon concentration vs. depth are shown in Figure III.la to e. The $\delta^{13} \mathrm{C}$ of the solid-phase organic carbon is also presented in these figures. In general, the concentration of organic carbon is about 20-24 $\mathrm{mg} \mathrm{C/gdw}$ in the surface and decreases regularly to a value of about $15-16 \mathrm{mg} \mathrm{C} / \mathrm{gdw}$ by $30 \mathrm{~cm}$. Data from the top $4 \mathrm{~cm}$ of the December 83 core are an exception to this trend. The concentrations of organic carbon are approximately $10 \mathrm{mg} \mathrm{C/gdw} \mathrm{higher} \mathrm{in} \mathrm{the} \mathrm{December} 83$ samples than in any other core. Isotopic data from all cores are very constant and averages $-20.6 \pm$ $0.2 \mathrm{o} / 00$; there is no significant change with depth. The isotopic data from the December 83 core do not reflect the anomaly evident in the concentration of organic carbon. 
Figure III.1. Measured values of SOC and $\delta^{13} \mathrm{C}-\mathrm{SOC}$. The SOC values are reported as \%OC; this number can be converted to $\mathrm{mgC} / \mathrm{gdw}$ by multiplying by 10 , i.e. $\operatorname{SOC}(\mathrm{mgC} / \mathrm{gdw})=10(\% \mathrm{CC})$. 

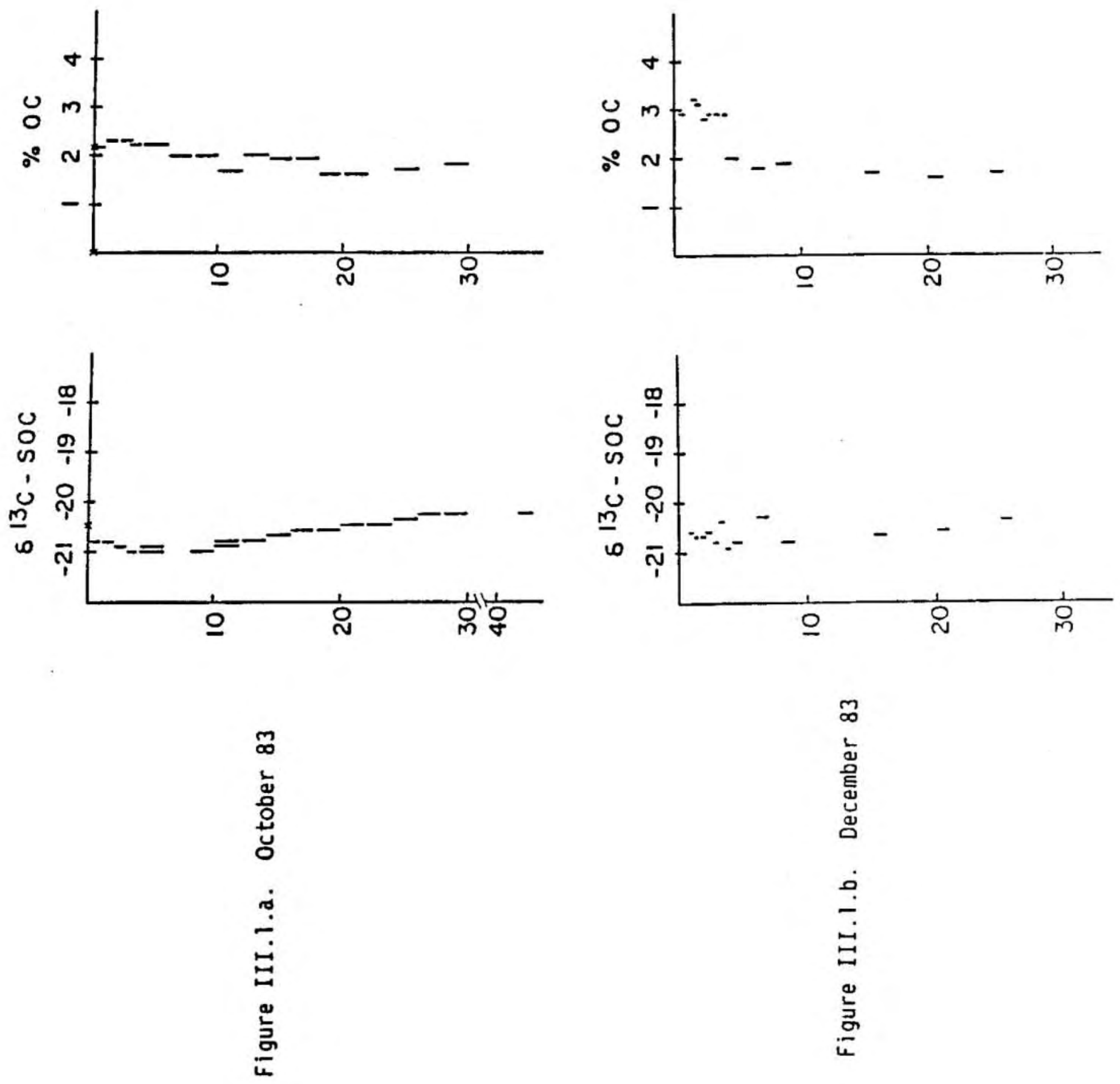

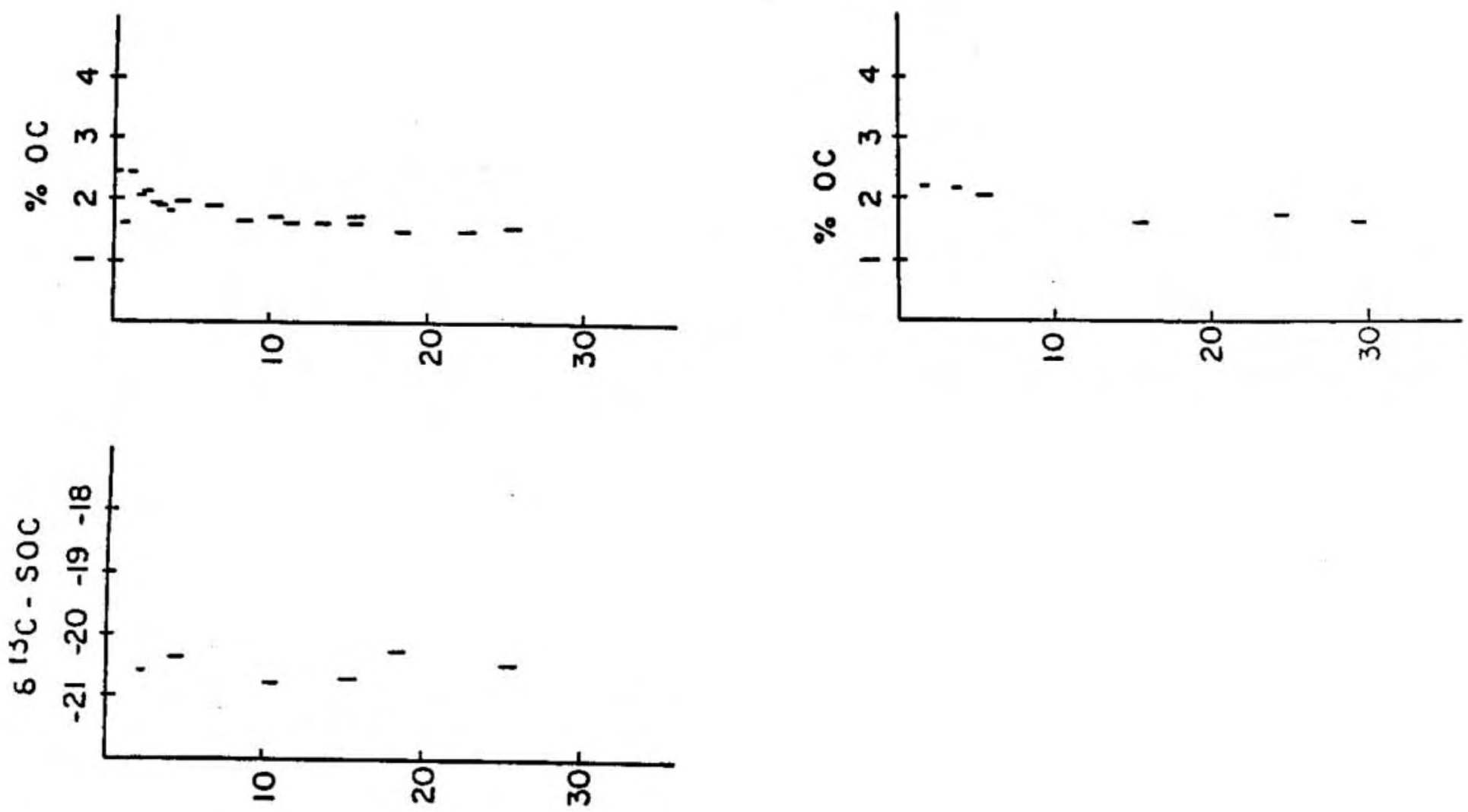

$\stackrel{+}{\infty}$
독

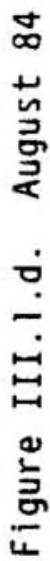




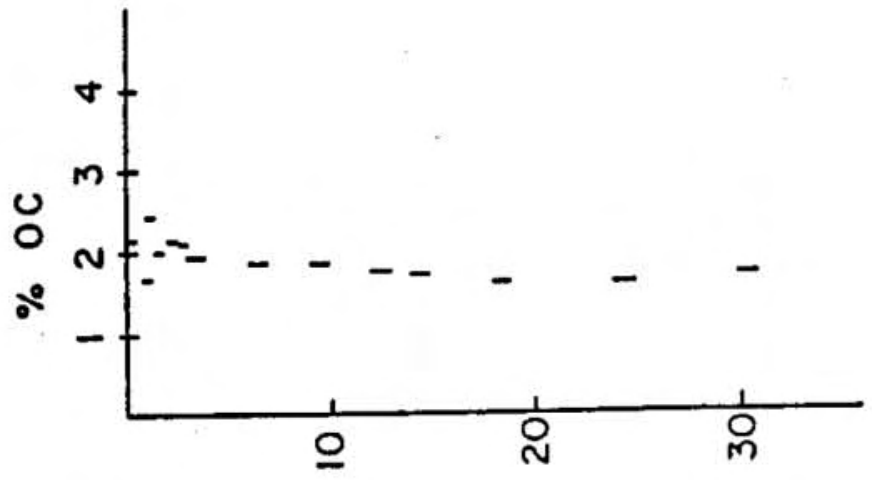

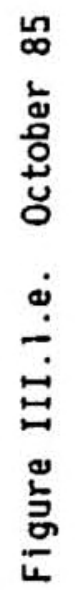




\section{III.1.B. Pore water data}

Depth profiles of $\delta^{13} \mathrm{C}-\sum \mathrm{CO}_{2}, \sum \mathrm{CO}_{2}, \mathrm{Alk}, \mathrm{Ca}, \mathrm{H}_{2} \mathrm{~S}, \mathrm{PO}_{4}$, and DOC are shown in Figures III.2 a to $f$ and listed in Table AI.I of Appendix I. Concentrations of $\left[\mathrm{CO}_{2}\right.$ were calculated from $\mathrm{pH}$ data in October 83 ; the $\mathrm{pH}$ data are listed in Table AI.1 in Appendix I. The constituents of most importance to the calculations made in this chapter are $\left[\mathrm{CO}_{2}, \mathrm{Alk}\right.$, and $\mathrm{Ca}$. In every month, the $\mathrm{SCO}_{2}$ concentration measured in the surface sediment interval (approximately 2.5 to $3.0 \mathrm{mM}$ ) is significantly greater than that measured in the bottom water $(2.0 \mathrm{mM})$; the concentration measured in the deepest sediment sample is greater than that measured in the surface sample. In some months there is a concentration maximum in the top 4-8 cm. In all except the March 85 core, Alk profiles are qualitatively similar to the $\left[\mathrm{CO}_{2}\right.$ profiles; the bottom water Alk is $2.0 \mathrm{meq} / 1$ and the surface pore water concentration is approximately 2.5 to $3.0 \mathrm{meq} / 1$. In March 85 , there is a minimum in the Alk profile in the upper $4 \mathrm{~cm}$. Dissolved $\mathrm{Ca}$ concentrations are always greater in pore water than in bottom water, and average around 9.3 $\mathrm{mM}$ in the pore waters. In most months the Ca concentration is constant with depth, but, in June 84 and March 85 , there is a maximum in the profile in the top $4 \mathrm{~cm}$. Dissolved $\mathrm{PO}_{4}$ concentrations range from 10 to $120 \mu \mathrm{M}$ and exhibit a maximum in the upper $4 \mathrm{~cm}$ of the sediment column. Dissolved sulfide is not detectable in the first $2 \mathrm{~cm}$ and is present at close to the detection limit from 2 to $4 \mathrm{~cm}$. Below $4 \mathrm{~cm}$, the dissolved sulfide concentration may increase to as much as $1 \mathrm{mM}$ by $30 \mathrm{~cm}$. DOC was measured in October 83 , December 83 , and June 84 . Its concentration ranges from 2 to $30 \mathrm{mg} \mathrm{C} / 1$, and there is a concentration maximum between 2 and $12 \mathrm{~cm}$. 
Figure III.2. Depth profiles of $\delta^{13} \mathrm{C}-\left[\mathrm{CO}_{2}, \sum \mathrm{CO}_{2}, \mathrm{Alk}, \mathrm{Ca}, \mathrm{H}_{2} \mathrm{~S}\right.$, $\mathrm{PO}_{4}$, and $\mathrm{DOC}$ for the six months sampled in this study. 

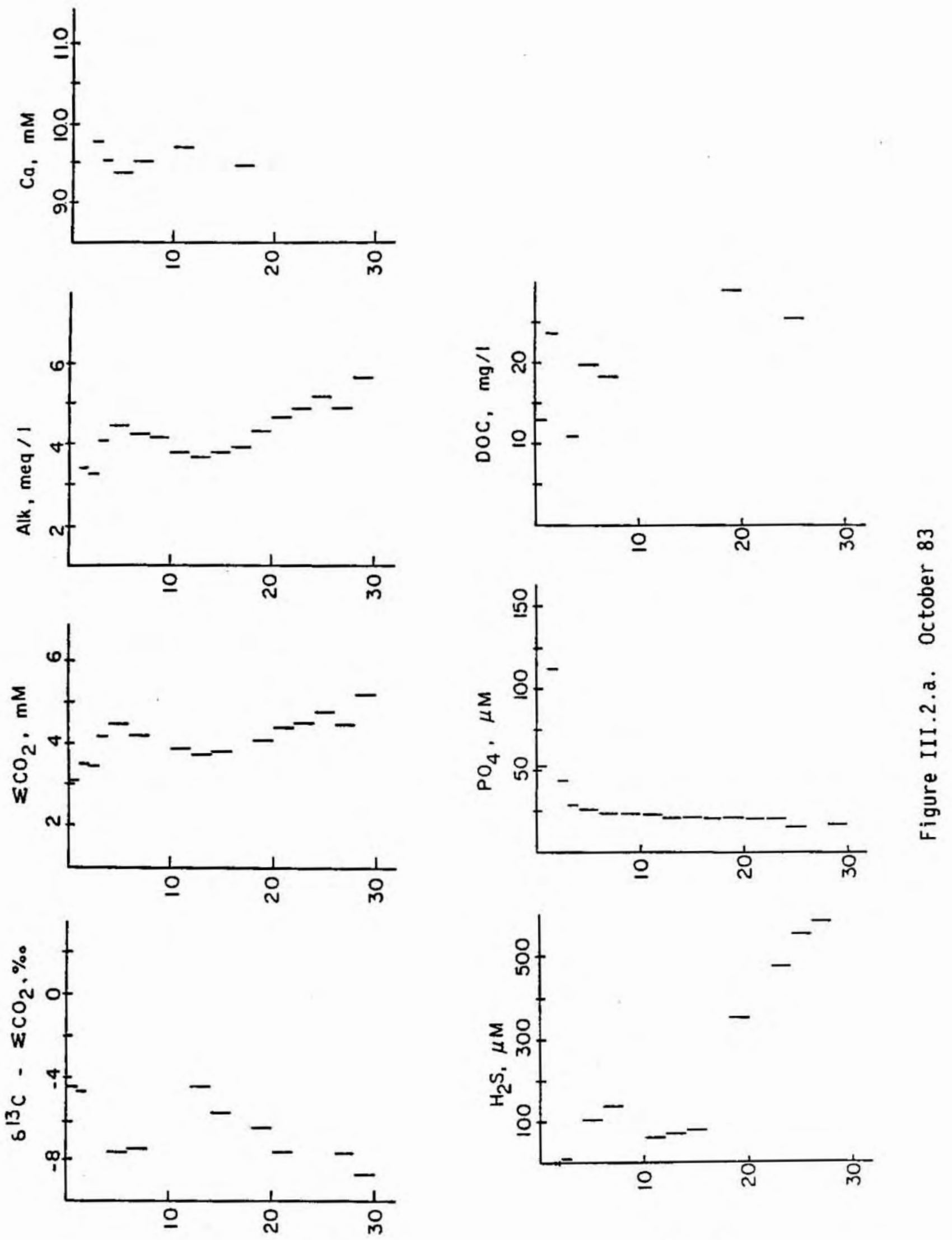

(ய) पावन्व

(யง) पाdəa 

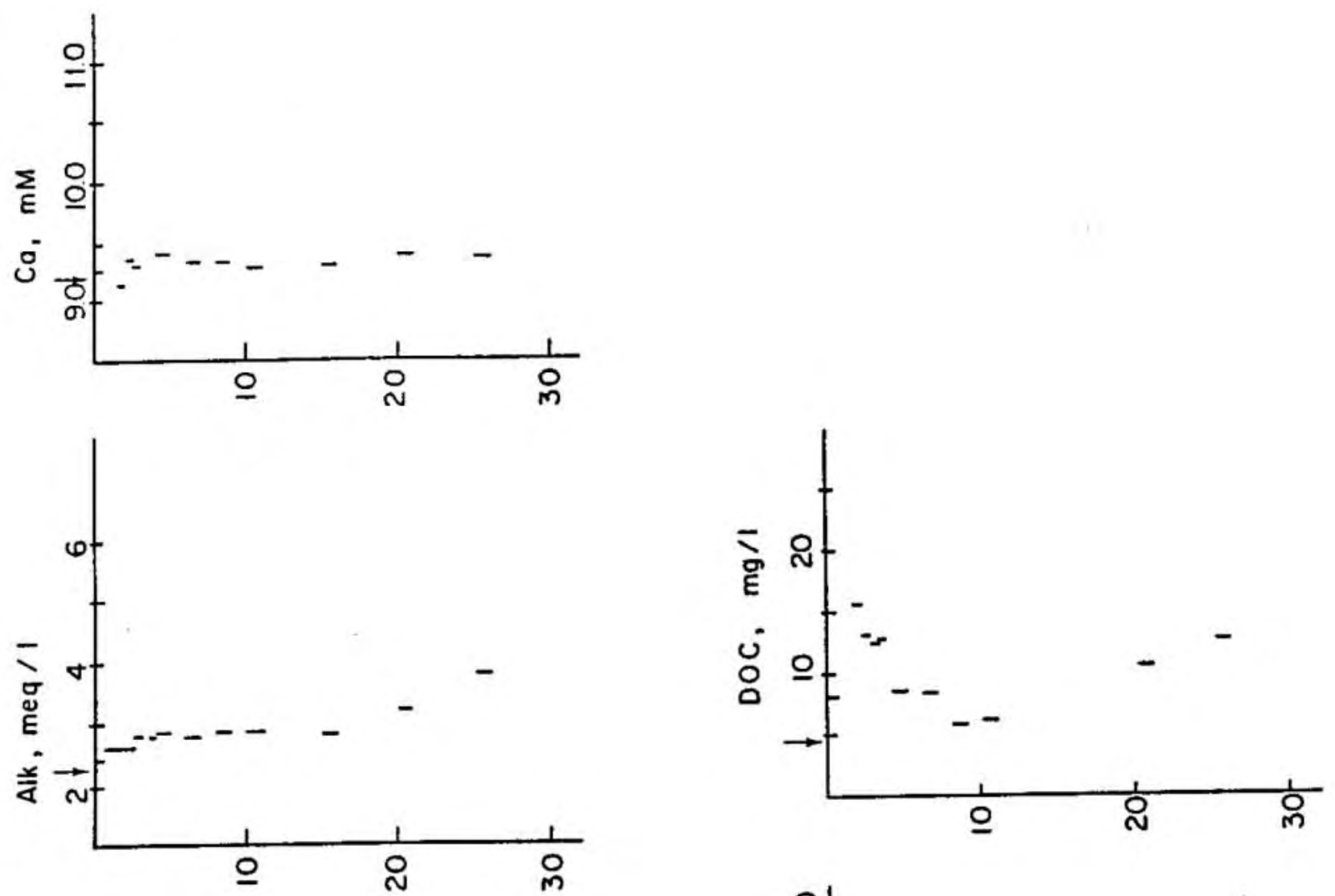

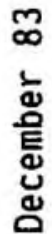
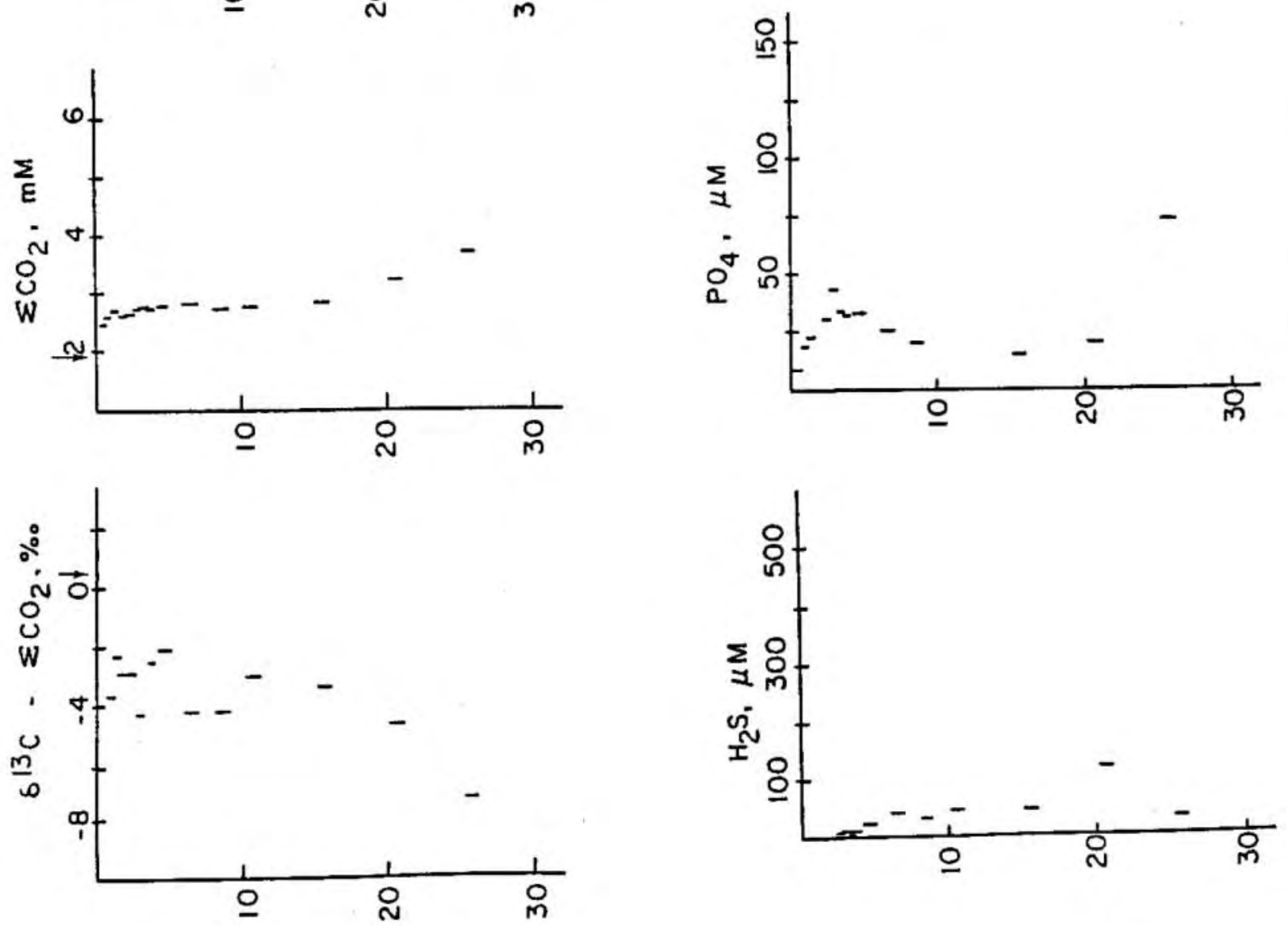

(யכ) पाdəO

(யग) पाdəO 

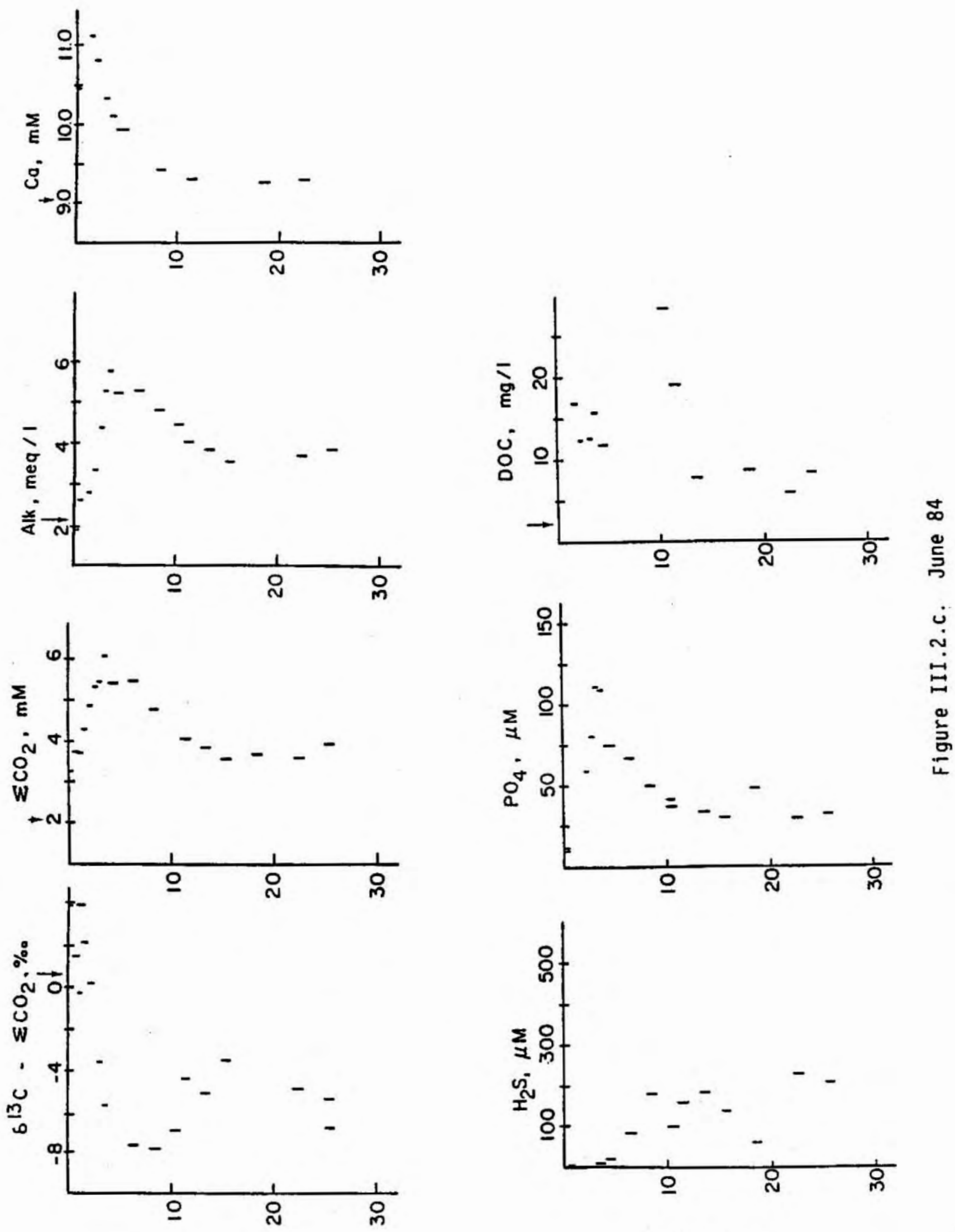

(யง) 41dəo

(w) ५।dəa 

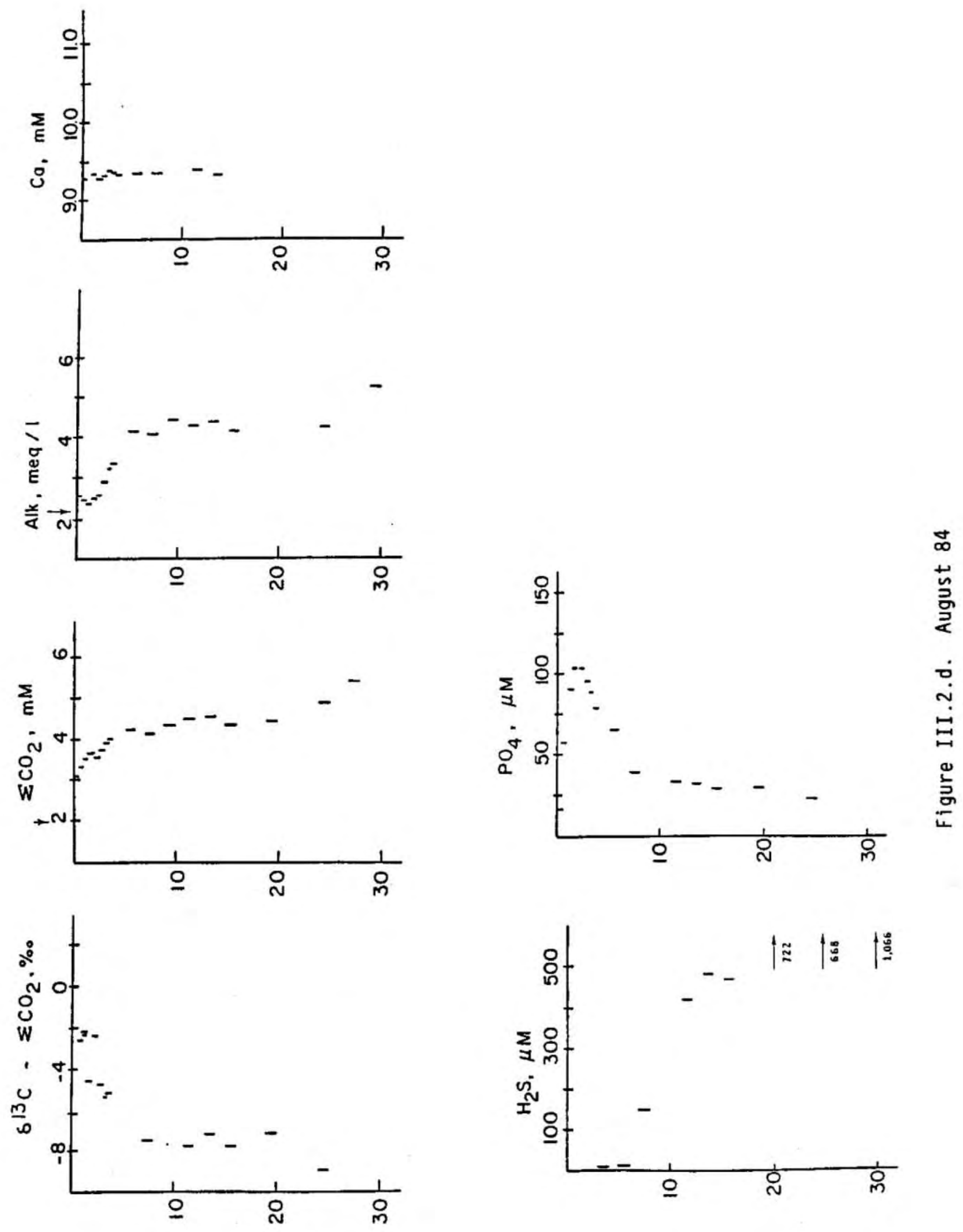

(ய) पाdन0

(யכ) पाdag 

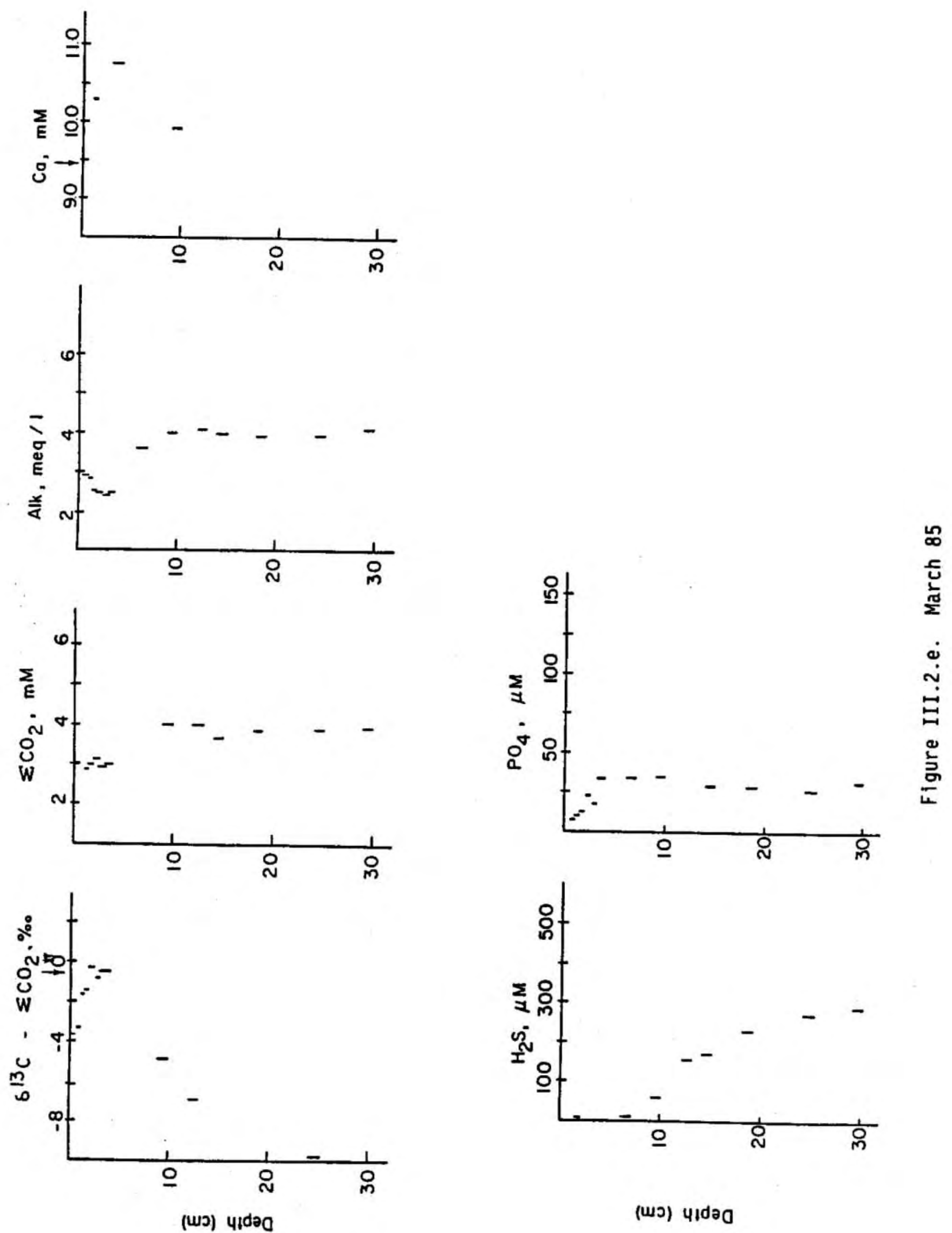

(யग) पाdə0 

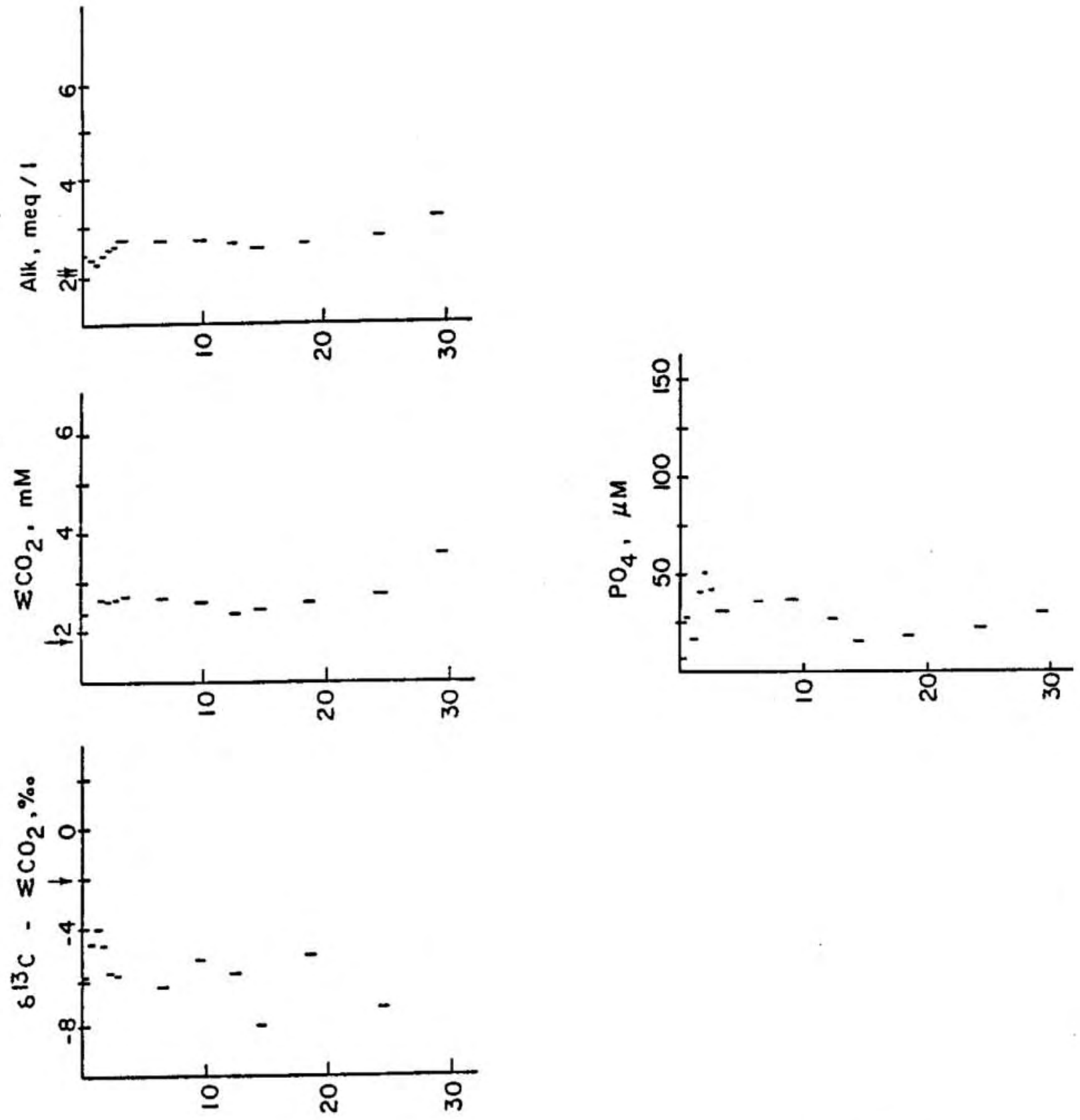

(wว) पाda्

(w) पाdə0 


\section{III.2. A Carbon Budget for Buzzards Bay Sediments}

III.2.A. Calculation of burial rate of organic carbon in the sediments

The amount of carbon buried in the sediments can be calculated from the concentration of $5 O C$ at depth $(16.0 \mathrm{mgC} / \mathrm{gdw})$ and the sedimentation rate calculated from ${ }^{14} \mathrm{C}$ data $(0.05 \mathrm{~cm} / \mathrm{yr}$, see Section V.4) if it is assumed that the oxidation of organic carbon in the sediments is negligible below $40 \mathrm{~cm}$. Based on oxidation rates calculated in this chapter, this is a valid assumption.

$$
\begin{aligned}
& C_{b}=(0.05)(16) \rho(1-\phi) 10=5.5 \mathrm{gC} / \mathrm{m}^{2}-\mathrm{yr} \\
& \text { where } \rho=\text { density of solids }=2.62 \mathrm{~g} / \mathrm{cm}^{3} \\
& \phi=\text { porosity at depth }=.737 \\
& \text { both from Martin (1985) }
\end{aligned}
$$

III.2.B. Calculation of rate of oxidation of organic matter

The profiles of $\left[\mathrm{CO}_{2}\right.$ and $\mathrm{SOC}$ with depth in the sediments can be used to estimate the rate at which organic carbon is oxidized in the sediments. Two diagenetic models that describe the major processes affecting the depth profiles of these constituents are developed in the following sections. A solution model will describe the profiles of $\left[\mathrm{CO}_{2}\right.$ with depth. Oxidation rates obtained from this model will be compared with those from a second, solid-phase model describing depth profiles of SOC. Oxidation rates calculated from the models can then be used to calculate the amount of organic carbon remineralized in the sediment column. 


\section{III.2.B.1. Solution Model}

Mathematically modelling the profile of $\mathrm{SCO}_{2}$ is complex unless several assumptions are made. In the next section, the assumptions necessary to formulate equations describing the behavior of $\mathrm{SCO}_{2}$ are presented. The concentration of $\mathrm{CCO}_{2}$ in pore waters is affected mainly by the oxidation of organic carbon, the dissolution or precipitation of $\mathrm{CaCO}_{3}$, and mixing with bottom water through irrigation. A two-zone model used to describe these processes is represented in Figure III.3.

Oxidation of organic carbon

The model will be developed with the oxidation of organic carbon described as a $\mathrm{CO}_{2}$ production rate, $R_{c}$. The sediment column is divided into two zones. $R_{c}$ will be assumed constant with depth in the surface zone and exponentially decreasing in the deeper zone. These assumptions are based on a knowledge of sulfate reduction rates in coastal sediments. In the sulfate-reduction zone, $\mathrm{CO}_{2}$ production is directly related to sulfate reduction. Howarth (unpublished) measured sulfate reduction rates in surface sediments from this site; his data showed that the sulfate reduction rate was constant between 2 and $10 \mathrm{~cm}$. In deeper sediments $(10-100 \mathrm{~cm})$ of Long Is land Sound similar to the Buzzards Bay site, Westrich (1985) used a series of exponentially decreasing functions to describe the sulfate reduction rate data.

Based on dissolved sulfide profiles (Figure III.2 a to f), it is unlikely that sulfate is the only oxidant in the top $4-10 \mathrm{~cm}$. The sediments at this site are overlain by a fully oxygenated water column and at certain times of the year there is significant irrigation af the surface sediments. Oxygen penetration is 1 imited by the rate at which it can diffuse in from the surface 
Figure III.3. Representation of the model used to describe the $\sum \mathrm{CO}_{2}$ data. 
Figure III.3

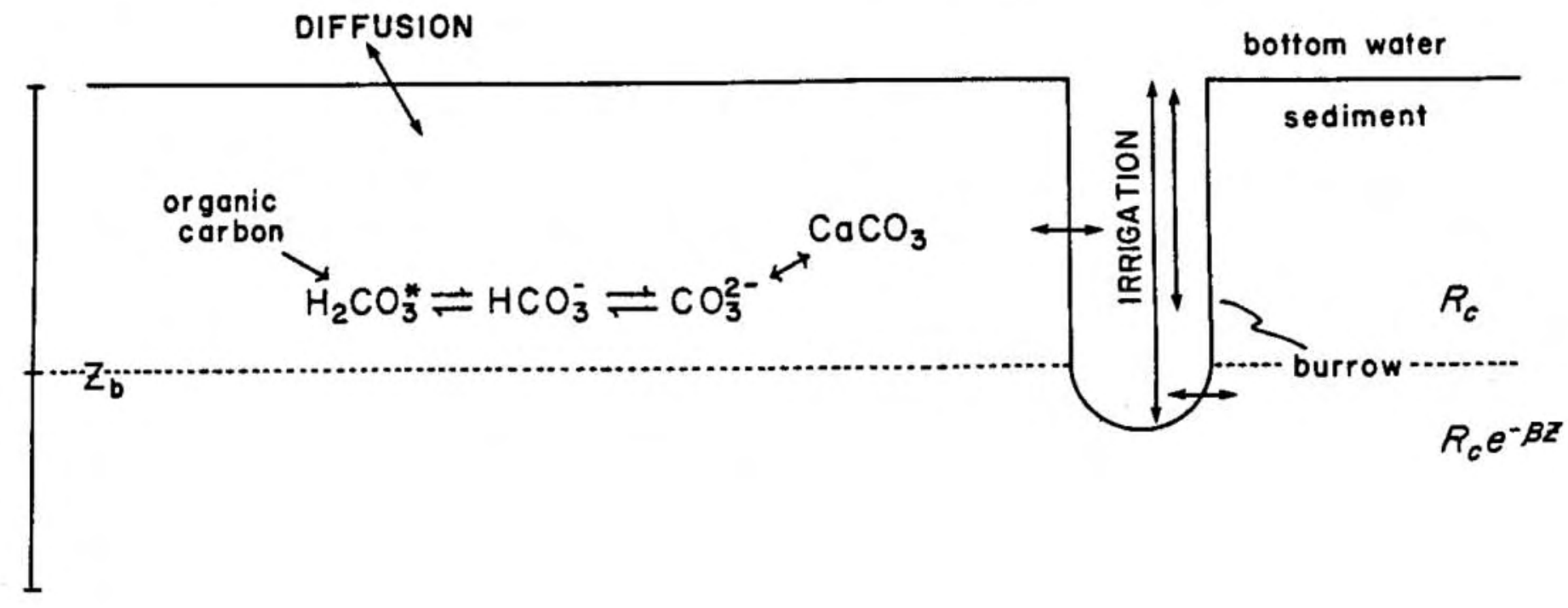


and through burrow walls. Nitrate is another oxidant that is sometimes present in Buzzards Bay pore waters (Henrichs, 1980). However, the concentration of nitrate is so low relative to the other oxidants in these sediments that it cannot be an important oxidant in the carbon budget. Dissolved iron and manganese profiles indicate that organic matter is oxidized by $\mathrm{MnO}_{2}$ and $\mathrm{Fe}_{2} \mathrm{O}_{3}$ in the surface sediments between 0 and $1.5 \mathrm{~cm}$ (Martin, 1985). The depth to which these oxidants are important varies seasonally, with the shallowest depths occurring in warmer months. The existence of microenvironments in sediments (Jorgensen, 1977b) makes it possible for oxygen and sulfate reduction to occur in the same sediment layer even though sulfate reducers are obligate anaerobes. It is likely that the absence of dissolved sulfide in the surface zone is the result of oxidation to sulfate by oxygen and precipitation as iron sulfides.

It is probable that oxygen and sulfate are both important electron acceptors in the surface sediments. Although the depth functionality of $R_{c}$ has been based on a knowledge of sulfate reduction rates, there is no assumption in the model concerning the electron acceptor used to produce $\mathrm{CO}_{2}$. In coastal sediments, where the supply of fresh organic carbon to the sediments is high and the solid-phase mixing rates are rapid, it is likely that the oxidation rate is dependent on the quality of the organic matter in the sediments (Westrich and Berner, 1984). As defined, $R_{c}$ can describe the oxidation of organic carbon in these sediments by a variety of electron acceptors.

Oxidation of organic carbon is controlled by some combination of the following factors: 1) the quality of the organic matter in the sediment 
column, 2) the location and size of the microbial population, and 3) the temperature. The $\mathrm{CO}_{2}$ production term used in this model does not differentiate between these factors. The production term also does not distinguish between production from the oxidation of organic carbon and from the dissolution of $\mathrm{CaCO}_{3}$.

Dissolution and precipitation of $\mathrm{CaCO}_{3}$

The dissolution of $\mathrm{CaCO}_{3}$ appears to be controlled by seasonal processes. and will be introduced into the carbon budget after the organic matter oxidation rate has been calculated (see Section III.2.C.). Precipitation of $\mathrm{CaCO}_{3}$ does not appear to be an important process in these sediments, although there is a loss of $\mathrm{Ca}$ from the dissolved phase in June 84 and March 85. The importance of the dissolution of $\mathrm{CaCO}_{3} \mathrm{can}$ be calculated from dissolved Ca data. Figure III.4 shows the calculated values of the ion concentration product (ICP) of dissolved $\mathrm{Ca}$ and $\mathrm{CO}_{3}^{2-}$ with depth as well as the equilibrium values for calcite and aragonite. The concentration of $\mathrm{CO}_{3}^{2-}$ was calculated from $\left[\mathrm{CO}_{2}, \mathrm{Alk}, \mathrm{PO}_{4}\right.$, and $\mathrm{H}_{2} \mathrm{~S}$ data. It is evident that the surface sediments are undersaturated with respect to $\mathrm{CaCO}_{3}$ at least two months of the year. Below $4 \mathrm{~cm}$, it appears that the pore waters are in equilibrium with some phase of $\mathrm{CaCO}_{3}$. The existence of disequilibrium in the surface sediments is not evidence that dissolution occurs; it indicates only that conditions favorable for dissolution exist. However, dissolved $\mathrm{Ca}$ concentrations provide evidence that dissolution of $\mathrm{CaCO}_{3}$ does occur in these sediments. The concentrations of $\mathrm{Ca}$ in the pore waters of the surface sediments are always greater than those in the bottom water, and in some months there is a pronounced maximum in the Ca profile 
Figure III.4. Calculated values of the ion concentration product [Ca] $\mathrm{CO}_{3}^{2-}$ ] and equilibrium values for calcite (solid line) and aragonite (dashed line). The value for calcite was calculated using the relationship given in Ingle et al. (1975), and the value for aragonite was assumed to be 1.5 times the calcite value (Aller, 1982). 
Figure III.4

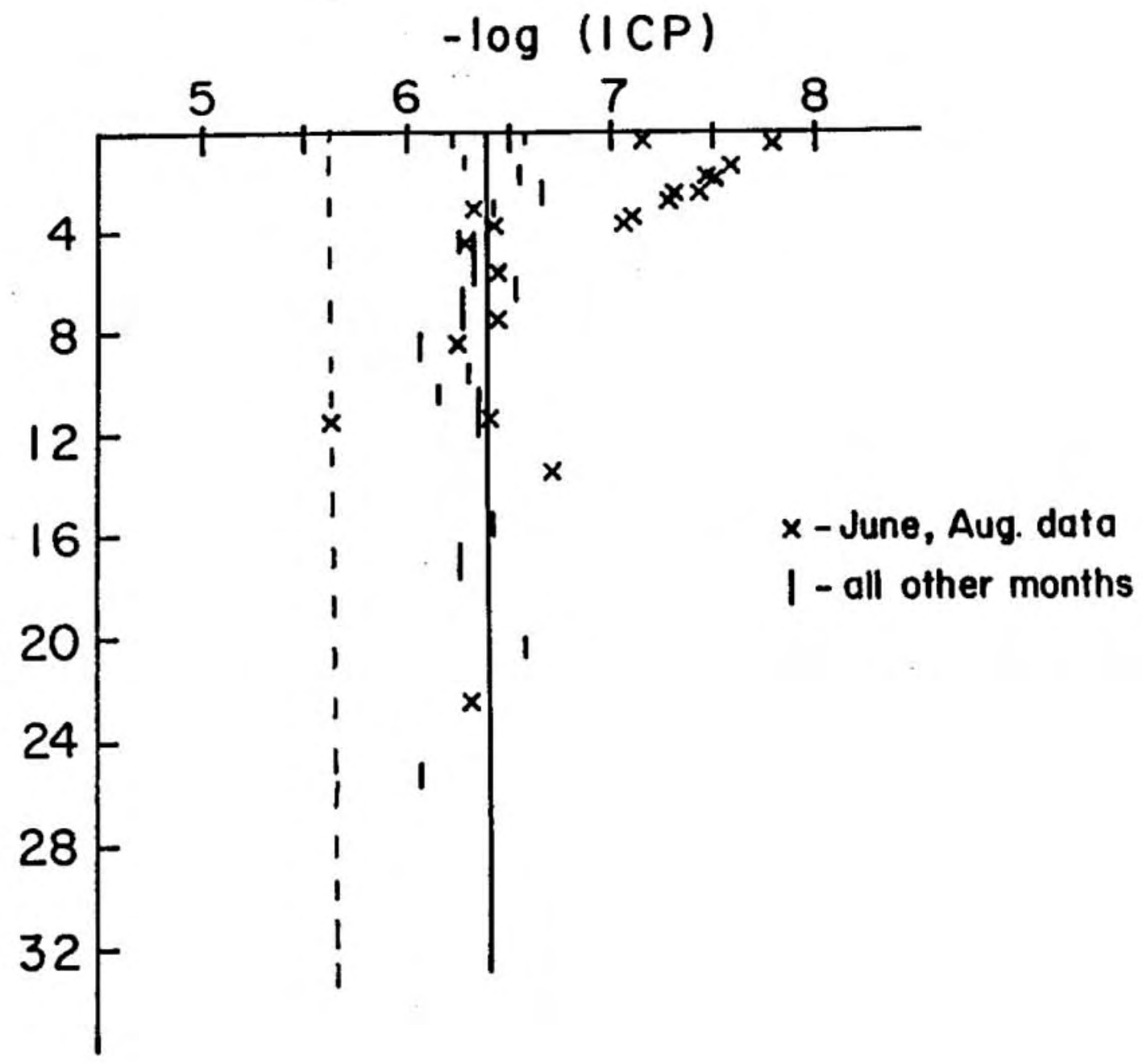


(see Figure III.2). These data indicate that there is always dissolution of $\mathrm{CaCO}_{3}$ at or near the sediment water interface and that, in some seasons, there is significant dissolution of $\mathrm{CaCO}_{3}$ within the sediment column as well. There is also visual evidence for the dissolution of $\mathrm{CaCO}_{3}$ in the sediments. Small clam shells taken from cores are obviously pitted and microscopic examination of the surface sediments reveals some severely eroded foraminifera shells.

The Ca data from June 84 and March 85 indicate that there was a loss of dissolved $\mathrm{Ca}$ at depth in the sediments. In June 84 , half of this loss can be accounted fro by irrigation. While there appears to be precipitation of some Ca mineral, it is unlikely to be $\mathrm{CaCO}_{3}$. Based on the curvature of the $\mathrm{Ca}$ profile in June 84 , some of the precipitation must occur in a region that is undersaturated with respect to $\mathrm{CaCO}_{3}$. It is possible that there is precipitation of an apatite phase. The zone of $\mathrm{Ca}$ consumption is coincident with a zone of $\mathrm{PO}_{4}$ consumption, and Jahnke et al. (1983) have proposed the precipitation of apatites in recent sediments.

Transport Parameters

Irrigation will be included in the solution model using the non-local source model of Emerson et al. (1984). The irrigation parameter, $\alpha$, can be estimated from the values calculated at this site by Martin (1985) using ${ }^{222} \mathrm{Rn} /{ }^{26} \mathrm{Ra}$ disequilibrium. He found that irrigation was an important transport mechanism for dissolved species in warm months and can be important to depths of at least $20 \mathrm{~cm}$. In colder months (December through March), the ${ }^{22} \mathrm{Rn}$ profiles were adequately described by considering molecular diffusion as the only transport process. The irrigation parameter will be assumed 
constant with depth in both zones although different values can be assigned for each zone as necessary. Martin (1985) found that, in certain months, $\alpha$ could be described best with an exponentially decreasing function while in other months it was constant with depth. For the months in which irrigation decreased with depth, over $90 \%$ of the integrated irrigation occured above 10 $\mathrm{cm}$. To calculate an $\alpha$ that is constant with depth from the exponential functions, The following average value is used:

$$
\bar{\alpha}_{z_{a}}-z_{b}=\left[\int_{z a}^{z b} \alpha_{0} e^{-z / \alpha \mid} d z\right] /\left(z_{b}-z_{a}\right)
$$

The integrand on the right-hand side of equation 3-1 is the exponential function used to describe the ${ }^{222} \mathrm{Rn}$ deficit. The value of $\alpha$ has been calculated assuming that transport across burrow walls is by molecular diffusion, and that there are no chemical reactions in the burrow walls and linings that impede $\sum \mathrm{CO}_{2}$ transport. Thus, it is assumed that the irrigational transport of ${ }^{222} \mathrm{Rn}$ and $\sum \mathrm{CO}_{2}$ differ only because of the difference in their molecular diffusion coefficients, i.e. $\alpha_{\Sigma \cos }=$ $\alpha_{R n}\left(D_{\sum \mathrm{CO}_{2}} / D_{R n}\right)$.

The sediment diffusion coefficient $\left(D_{s}\right)$ is assumed to be constant with depth. Sediment diffusion coefficients were calculated from molecular diffusion coefficients for seawater $\left(D_{m_{0}}\right)$ with the following equation:

$$
\begin{aligned}
D_{s}=D_{m o 1} / \theta^{2} & \\
& \text { where } \theta=\text { tortuosity of the sediments }
\end{aligned}
$$

and

$$
\begin{aligned}
& \theta=\phi\left(R / R_{0}\right) \\
& \text { where } \phi=\text { porosity } \\
& R / R_{0}=\text { ratio of resistivity of bulk sediment to }
\end{aligned}
$$




\section{interstitial fluid}

The value of $R / R_{0}$ in these sediments is $2.0 \pm 0.2$ between $2-30 \mathrm{~cm}$ (Sayles, personal communication), and the average porosity between $0-30 \mathrm{~cm}$ is 0.767 . Thus

$$
0_{3}=0.650_{\mathrm{mol}}
$$

The molecular diffusion coefficients for $\mathrm{HCO}_{3}^{-}$and $\mathrm{Ca}$ in seawater reported by Li and Gregory (1974) were used, and corrected to observed temperatures. The dissolved Ca profile in sediments may also be affected by adsorption to clay surfaces; under certain conditions, consideration of this process may significantly change the effective diffusion coefficient. The effect of adsorption on the carbon budget calculation will be discussed in Section III.2.C. Values of $\mathrm{D}_{\mathrm{s}}$ calculated for each month are listed in Table III.1.

The sediments are assumed to have a constant porosity. This assumption is not true; Martin (1985) has described the porosity of these sediments with the following equation:

$$
\phi=0.737+0.159 \mathrm{e}^{-0.1872}
$$

However, most of the porosity change occurs in the upper $4 \mathrm{~cm}$ and neglecting this change will not alter the conclusions drawn from the model.

Mathematical Model

Given these assumptions the equations describing the model are:

$$
\begin{array}{lll}
\text { I } & \partial C / \partial t=D_{c}\left(\partial^{2} C / \partial z^{2}\right)-\alpha^{*}\left(C-C_{0}\right)+R_{c} & 3-3 a \\
\text { II } & \partial C / \partial t=D_{c}\left(\partial^{2} C / \partial z^{2}\right)-\alpha^{*}\left(C-C_{0}\right)+R_{c} e^{-\beta z} & 3-3 b
\end{array}
$$

To analytically solve this set of equations, the $\sum \mathrm{CO}_{2}$ profile is assumed to be in steady-state. However, as seasonal changes are seen in the $\sum \mathrm{CO}_{2}$ profiles, the validity of this assumption must be examined. In a 
Table III.1. The values of $\mathrm{D}_{s}$ for $\mathrm{HCO}_{3}^{-}, \mathrm{Ca}$, and $\mathrm{Rn}$ ions used in model and flux calculations for each month. See text for explanation of how $D_{H} c_{3}$ and $D_{c a}$ were calculated. The values of $D_{R n}$ were taken from Martin (1985).

\begin{tabular}{|c|c|c|c|c|}
\hline \multirow[b]{3}{*}{ Core date } & \multicolumn{4}{|l|}{ Bottom } \\
\hline & \multirow{2}{*}{$\begin{array}{l}\text { Water } \\
\text { Temp. }{ }^{\circ} \mathrm{C}\end{array}$} & \multicolumn{3}{|c|}{$D_{s} \times 10^{6}, \mathrm{~cm}^{2} / \mathrm{sec}$} \\
\hline & & $\underline{D}_{\mathrm{HCO}}$ & $\underline{D}_{c \mathrm{a}}$ & $\underline{D}_{R \cap}$ \\
\hline Oct. 17,83 & 15 & 6.4 & 4.1 & 6.7 \\
\hline Dec. 16,83 & 7 & 5.3 & 3.4 & 5.4 \\
\hline June 20,84 & 16 & 6.5 & 4.2 & 6.9 \\
\hline Aug. 30,84 & 21 & 7.1 & 4.6 & 7.7 \\
\hline March 26,85 & 4 & 5.0 & 3.2 & 4.9 \\
\hline Oct. 30,85 & 14 & 6.2 & 4.0 & 6.5 \\
\hline
\end{tabular}


study of Long Island Sound sediments, Aller (1977) used a radial diffusion model to interpret the data. The steady-state case was the only one for which he solved the equations. He found that the basic features of time dependence in profile shape could be produced by the temperature dependence of reworking and metabolic activity. The short diffusion distance from sediment to burrow channel in the radial diffusion model was thought to be the reason for the rapid re-establishment of steady-state. The description of irrigation in the non-local source model is analogous to the radial diffusion model (Boudreau, 1984), and the above argument should be valid for this model too.

To check on the validity of the steady-state assumption, a single equation that approximates the system described above was solved numerically using the Crank-Nicholson method (Crank, 1956). In this approximation irrigation is assumed to be constant and equal over the depth interval considered and $R_{c}$ decreases exponentially over the entire depth interval, i.e.

$$
\partial C / \partial t=D_{c} \partial^{2} C / \partial z^{2}-\alpha\left(C-C_{0}\right)+R_{c} e^{-\beta 2} \quad 3-4
$$

Using just one equation, both irrigation and $R_{c}$ will be underestimated at the surface and overestimated at depth. The equation was solved for a $20 \mathrm{~cm}$ depth interval assuming the following initial and boundary conditions:

$$
\begin{aligned}
& t=0, C=C_{0} \text { at all } z \\
& t>0, z=20, C=C_{f} ; \text { and } t>0, z=0, c=C_{0}
\end{aligned}
$$

When this equation was solved using parameters that describe conditions similar to June $84,5.25$ months were required to reach steady-state conditions. However, in just two weeks the profile maximum was at $65 \%$ of its steady-state value and in four weeks it had reached $80 \%$ of this value. Figure 
III. 5 shows the approach to steady-state. It is important to note that the boundary condition assuming that $C=C_{0}$ at $t=0$ is an extreme case. It is unusual for the sediments to be washed out to bottom water concentrations. In eleven cores collected between December 82 and October 85 , only two have shown evidence that the pore waters were flushed to a significant extent with bottom water (Martin, 1985; this study). Equation 3-4 was solved for two other cases; these solutions are shown in Figure III.6 a and b. Figure III.6 a indicates how long it might take to go from early summer to late summer conditions, e.g. June 84 to late August 84 . Again, actual steady-state concentrations are not achieved until 5.75 months have elapsed, but in four weeks the calculated profile is very close to the steady-state profile. The predicted concentration maximum of $7.5 \mathrm{mM}\left[\mathrm{CO}_{2}\right.$ is much greater than any concentration observed in the field. This is because irrigation in the surface sediments is underestimated greatly in the one-layer model. If the 4 week profile is modelled as though it were the steady-state profile, the value of $R_{c}$ in the surface sediments is underestimated by $25 \%$ and the total amount of carbon oxidized in the top $20 \mathrm{~cm}$ is underestimated by $20 \%$. In Figure III. 6 $b$, the case of going from an established summer profile to winter conditions is examined. In this solution, $\alpha$ has been assigned a value that is essentially zero. Even after nine months, steady-state concentrations have not been achieved in the deeper sediments. In this case, if $\alpha$ is maintained at a negligibly small value, the 4 week profile cannot be modelled as steady-state unless $R_{c}$ is also very small ( $\left.<1 \times 10^{-10} \mathrm{~mol} / \mathrm{L}-\mathrm{sec}\right)$. Thus, while the steady-state assumption is an approximation, reasonable results can 
Figure III.5. Approach of the time-dependent numerical model to steady-state, assuming that at $t=0, C=C_{0}$ for all $z$. In about 4 weeks the profile is approaching the steady-state solution. 
Figure III.5

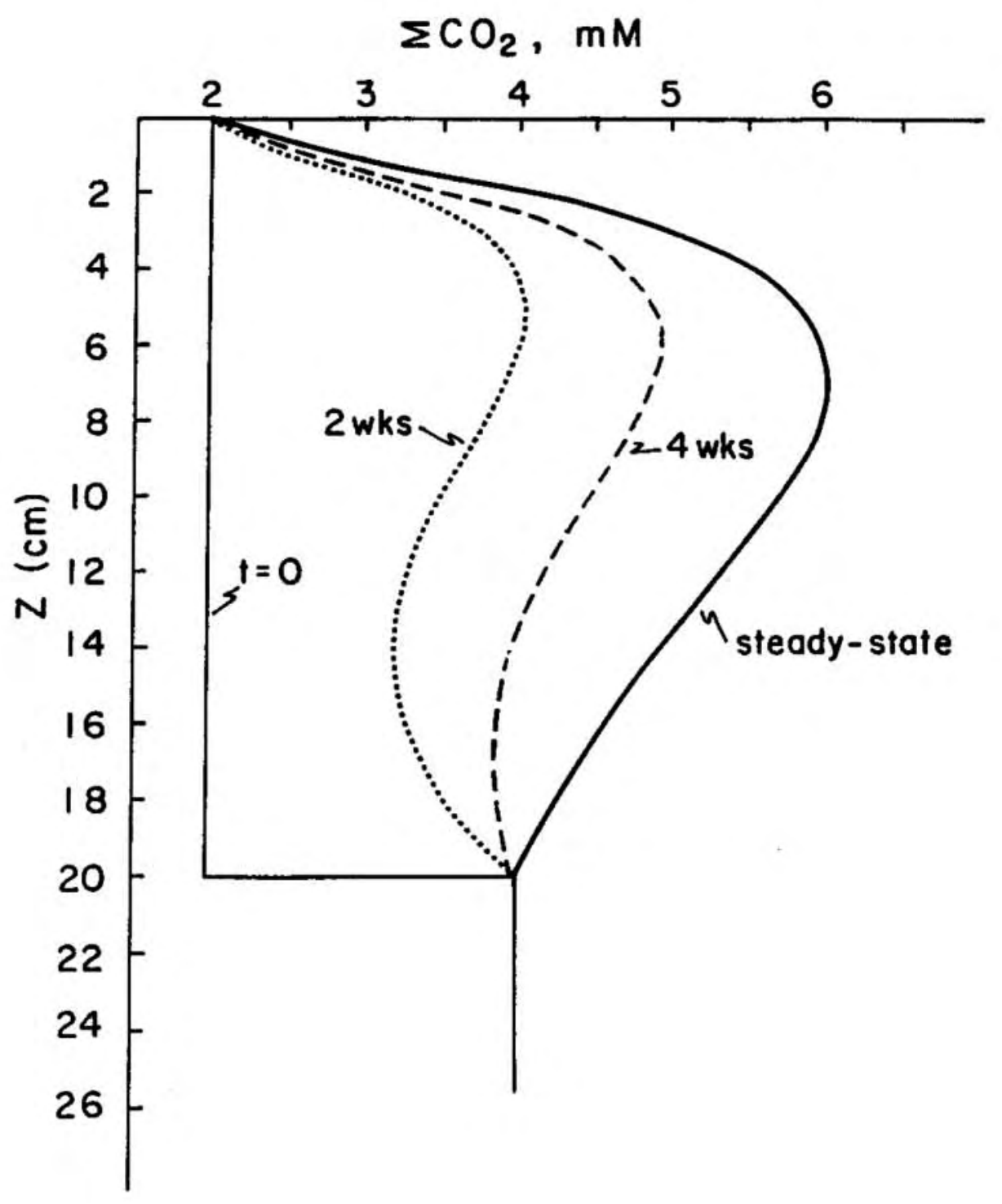


Figure III.6. Approachs to steady-state concentrations of $\sum \mathrm{CO}_{2}$ using a one-layer model and assuming an initial steady-state representative of early summer conditions.

a. early summer to late summer conditions, initial steady-state conditions:

$\alpha=3.5 \times 10^{-7}, D_{\mathrm{HCO}_{3}}=6.5 \times 10^{-6}, \beta=.1, R_{\mathrm{c}}=3.0 \times 10^{-9}$;

time-dependent conditions: $\alpha=4.5 \times 10^{-7}, D_{\mathrm{HCO}_{3}}=7 \times 10^{-6}, \beta=.08$,

$R_{c}=6.0 \times 10^{-9}$

b. early summer to winter conditions, initial steady-state conditions: $\alpha=$ $3.5 \times 10^{-7}, D_{\mathrm{HCO}_{3}}=6.5 \times 10^{-5}, B=.1, R_{c}=4.0 \times 10^{-9}$;

time-dependent conditions: $\alpha=1.0 \times 10^{-10}, D_{\mathrm{HCO}_{3}}=5 \times 10^{-6}, \beta=.1$, $R_{c}=0.6 \times 10^{-9}$ 


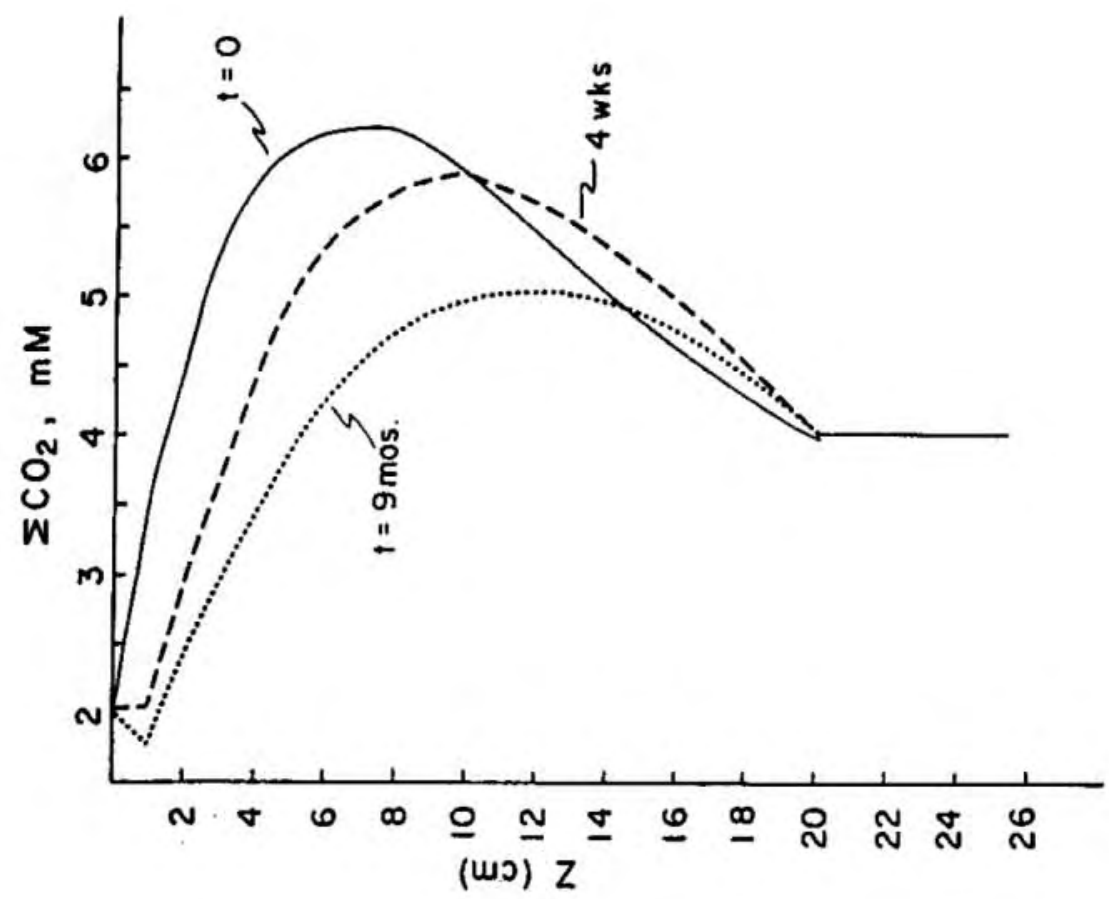

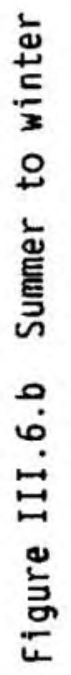

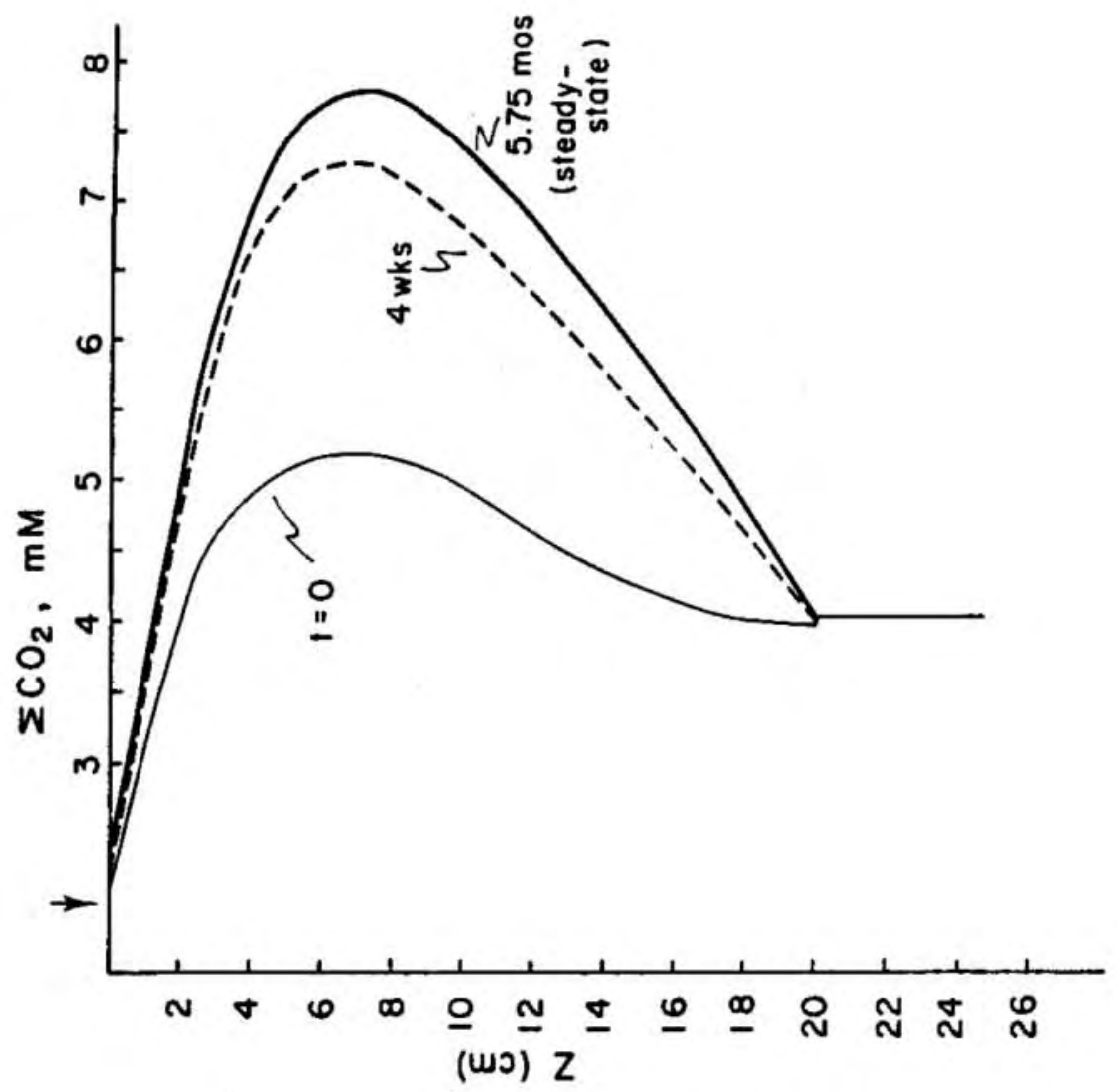

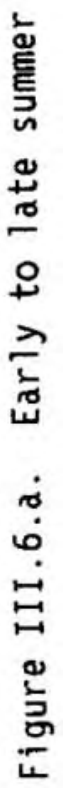


be obtained by using it at certain times of the year. This will be demonstrated further by comparing the model-calculated rates to independently estimated rates.

Using the steady-state approximation, the solutions to equations $3-3$ a and $b$ are:

$$
\begin{aligned}
& \text { I } C_{1}=C_{1} e^{\gamma 1 z}+C_{2} e^{-\gamma 1 z}+C_{T 0}+A_{1} \\
& \text { II } C_{11}=C_{4} e^{-\gamma 2 z}-A_{2} e^{-\beta z}+C_{0}
\end{aligned}
$$

where

$$
\begin{aligned}
& \gamma_{1}=\left(\alpha_{1} / D_{c}\right)^{1 / 2} ; \gamma_{2}=\left(\alpha_{2} / D_{c}\right)^{1 / 2} \\
& A_{1}=R_{c} / \alpha_{1} ; A_{2}=R_{c} /\left(D_{c} B^{2}-\alpha_{2}\right)
\end{aligned}
$$

Applying the following boundary conditions:

$$
\begin{aligned}
z & =0, C_{T}=C_{T 0} \\
z & =z_{b}, \quad C_{T 1}=C_{T 2} \quad \text { where } z_{b} \text { is boundary between I and II } \\
J_{1}=J_{11} & \\
z & \rightarrow \infty, d C_{1,} / d z \rightarrow 0 .
\end{aligned}
$$

the constants are:

$$
\begin{aligned}
& C_{1}=-\left(C_{2}+A_{1}\right) \\
& C_{2}=\frac{\left.A_{1} e^{\gamma / 2 b}\left(1+\gamma_{1} / \gamma_{2}\right)+A_{2} e^{-\beta 2 \gamma\left(\beta / \gamma_{2}\right.}-1 .\right)-A_{1}}{e^{-\gamma 1 \times b}\left(1-\gamma_{1} / \gamma_{2}\right)-e^{\gamma / 2 b}\left(1+\gamma_{1} / \gamma_{2}\right)} \\
& C_{4}=\frac{B A_{2} e^{-\beta 2 b}+\gamma_{1} C_{2}\left(e^{-\gamma 1 \times b}+e^{\gamma 121}\right)+\gamma_{1} A_{1} e^{\gamma 1 \times b}}{\gamma_{2} e^{-\gamma 22 b}}
\end{aligned}
$$

III.2.B.2. Solution Model Results

The model fits to data from October 83 , June 84 , and August 84 are shown in Figure III.7 a to $c$. The parameters used to fit the data are listed in 
Figure III.7. Steady-state model fits to data from October 83 , June 84 , and August 84. The parameters used to fit the data are listed in Table III.2. The solid lines represent the best fit. The dotted lines were calculated using values of $R_{c}$ two times the best-fit values, and the dashed lines were calculated using values of $R_{c}$ one-half the best-fit values. 


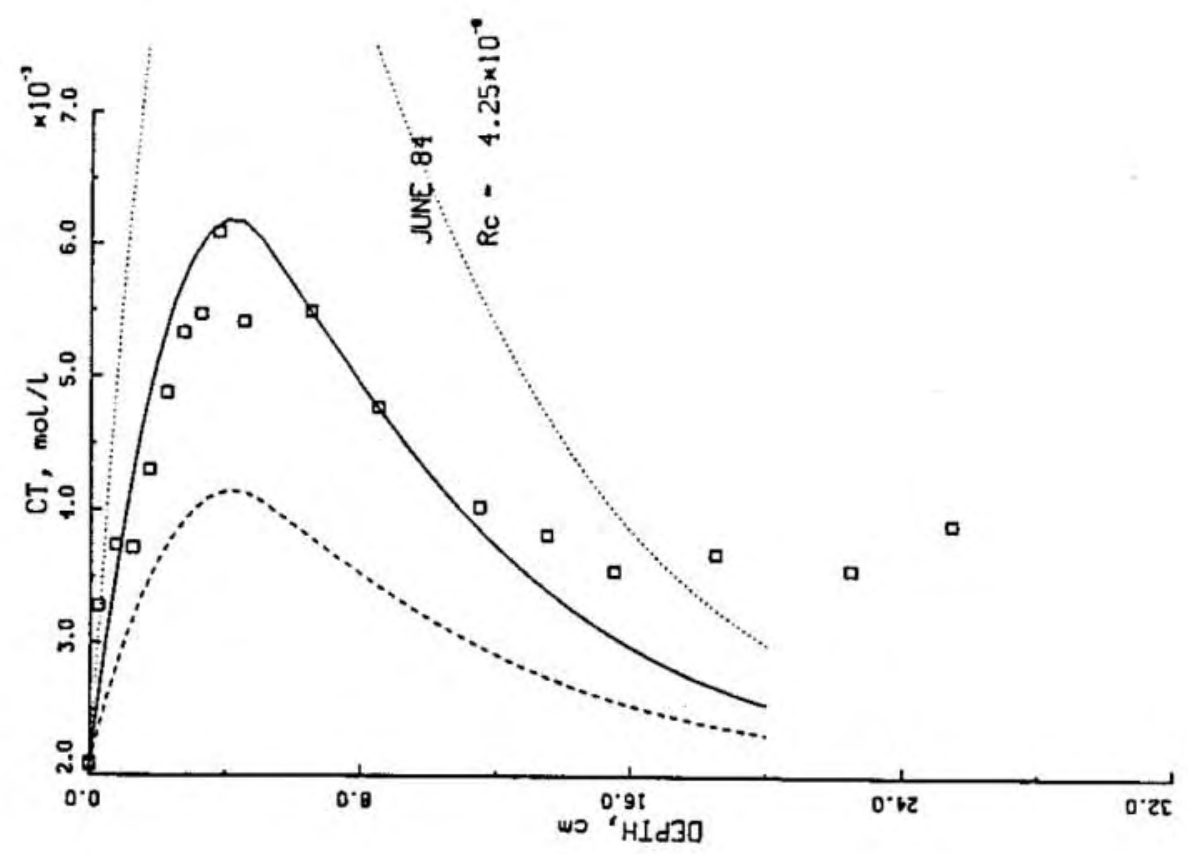

芒

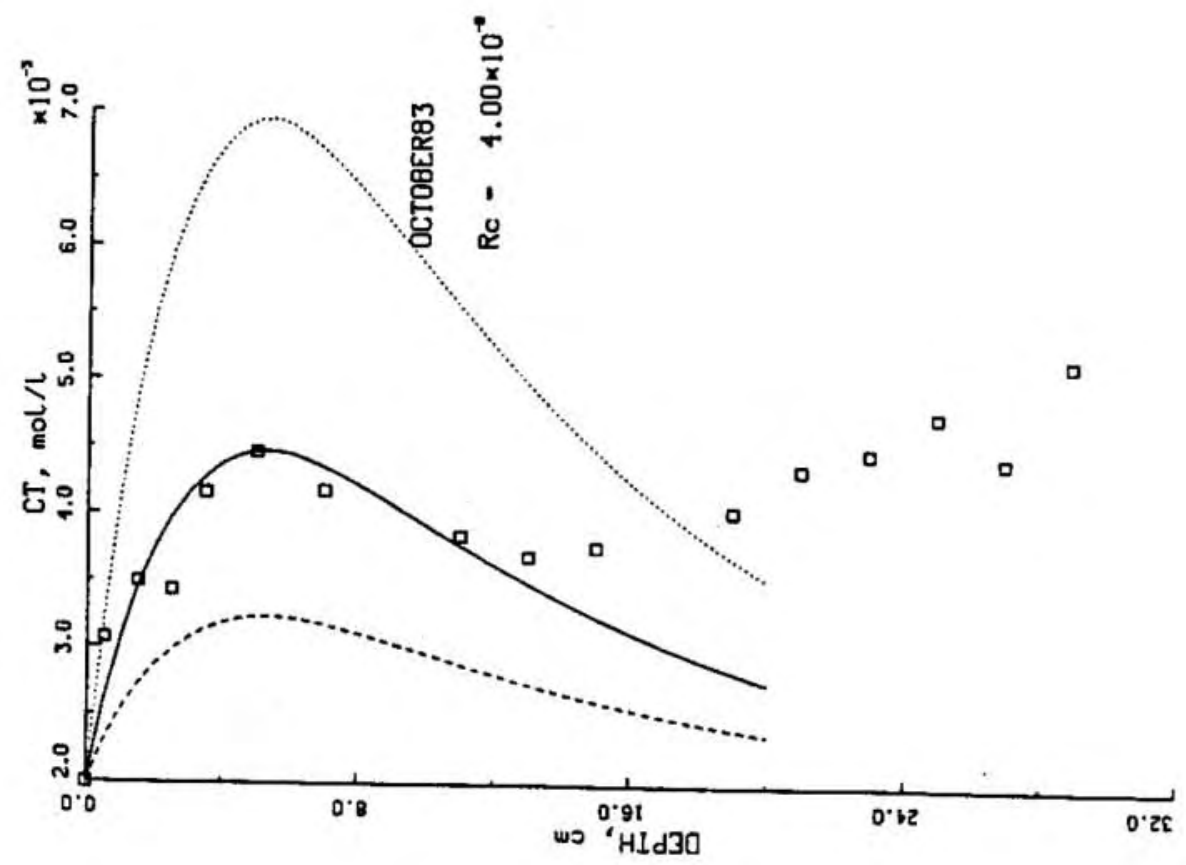

芒 


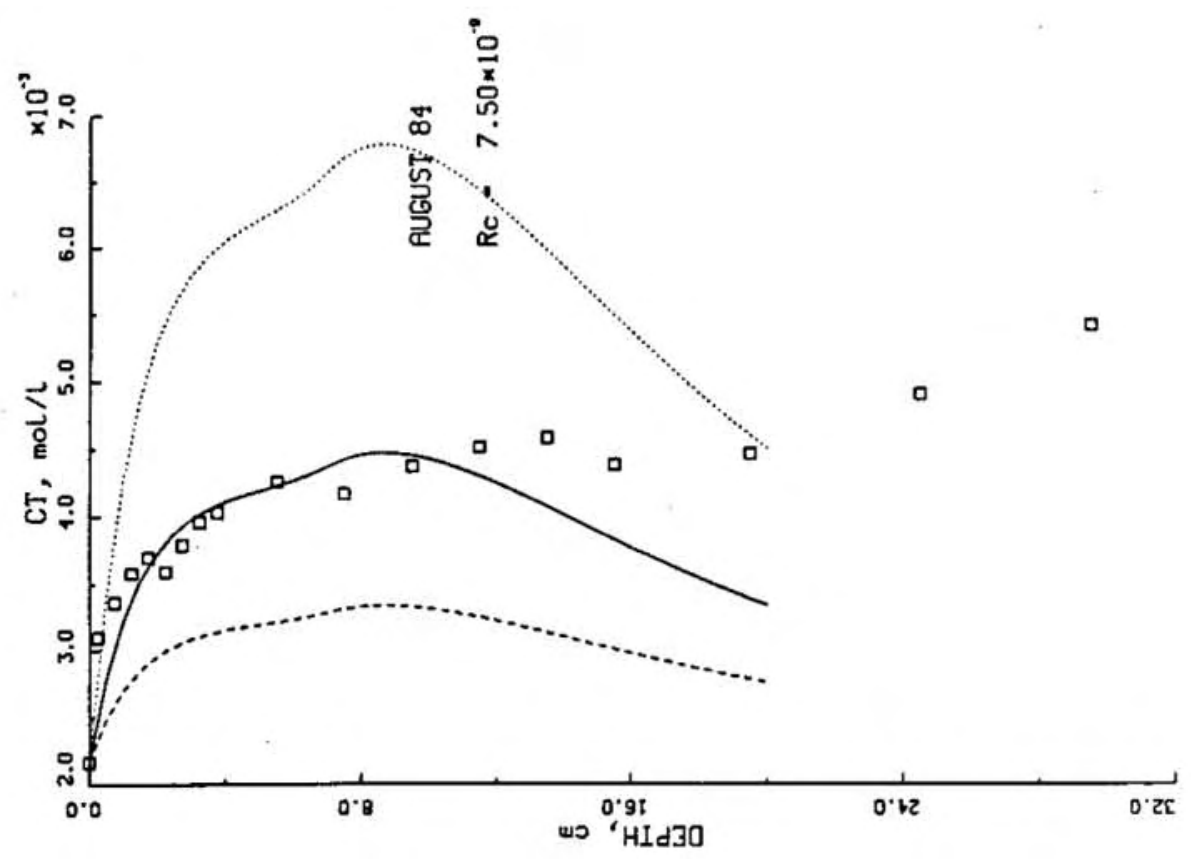

불 
Table III.2. These fits are not unique but are the ones judged best considering available knowledge about irrigation at this site and sulfate reduction rates in locations similar to this site. The model fit is shown only to $20 \mathrm{~cm}$ because this is the depth to which ${ }^{222} \mathrm{Rn} /{ }^{226} \mathrm{Ra}$ disequilibrium has been measured. The model-calculated profile always underestimates the amount of $\mathrm{SCO}_{2}$ in the sediments at depth. The most likely explanations for this are that 1) the model overestimates irrigation in the deeper sediments, and 2) there is more production at depth than the exponentially decreasing $\mathrm{CO}_{2}$ production rate accounts for. It is certain that the model is overestimating irrigation at depth. The irrigation term in the second zone can be smaller than that in the first zone, yet it is considered to be constant with depth. In other words, the model assumes that there is a small, but significant, amount of irrigation to infinite depths in the sediment, although this is known to be false for $\mathrm{Rn}$. The increase observed in the $\left[\mathrm{CO}_{2}\right.$ profile below $20 \mathrm{~cm}$ must be due to diffusion from a zone of production deeper in the sediment. Sulfate reduction should continue in the sediments until all the sulfate has been consumed; below this depth methanogenesis occurs (Reeburgh, 1982). At the interface between the sulfate reduction and the methanogenesis zones, there is a zone of methane oxidation (Reeburgh, 1982). The cessation of irrigation, continued sulfate reduction, and methane oxidation should create an increase in $\left[\mathrm{CO}_{2}\right.$ at depth in the sediment.

The effects of overestimating irrigation and underestimating $\mathrm{CO}_{2}$ production at depth in the sediments on carbon budgets calculated from this model should counteract each other. Overestimating irrigation in zone II 
Table III.2. The model parameters used to fit the data from October 83, June 84, and August 84. $R_{c}$ is reported in units of mol/L-sec, $\beta$ in $\mathrm{cm}^{-1}, \alpha$ in $\mathrm{sec}^{-1}$, and $z_{b}$ in $\mathrm{cm}$. The model is described in the text.

$\begin{array}{llllll}\text { Month } & \underline{R}_{\mathrm{c}} \times 10^{9} & \underline{\beta} & \underline{\alpha}_{1} & \underline{\alpha}_{2} & \underline{\underline{z}}_{0} \\ \text { Oct. } 83 & 4.0 & .35 & 1.4 \times 10^{-6} & 6.7 \times 10^{-8} & 6 \\ \text { June } 84 & 4.25 & .2 & 3.7 \times 10^{-7} & 3.7 \times 10^{-7} & 5 \\ \text { Aug. } 84 & 7.5 & .3 & 4.0 \times 10^{-6} & 6.0 \times 10^{-8} & 7\end{array}$


means that $\mathrm{CO}_{2}$ production in zone II must be increased near $\mathrm{z}_{\mathrm{b}}$ in order for the modelled profile to fit well at $z_{b}$. This should compensate for some of the underestimation due to ignoring production at depth. However, the overall effect of these two simplifications in constructing a carbon budget for this site is small when irrigation is not constant with depth. In October 83 and August 84 , the amount of carbon oxidized below $z_{b}$ was less than $10 \%$ of the total amount oxidized in the sediments. However, in June 84 , when irrigation was constant to $20 \mathrm{~cm}$, the amount of carbon oxidized below $z_{b}$ was almost $30 \%$ of the total amount oxidized. This indicates that the model parameters $R_{c}$ and $B$ are very sensitive to the values of $\alpha$ that are used. The actual value chosen for $R_{c}$ is dependent on the value chosen for the irrigation parameter, $\alpha$. Thus, if $\alpha$ is known to a certainty of $\pm 20 \%$, then $R_{c}$ is known to this certainty also. The values chosen for $\alpha_{1,2}$ in October 83 were based on values reported for October 82 , and those chosen for June 84 were based on values reported for June 84 (Martin, 1985). The values for $\alpha_{1,2}$ used in August 84 are greater than any measured at this site. However, it was not possible to reproduce the observed profile in the top 10 cm without using a large irrigation term. Accurate estimates of the oxidation rate depend on a good knowledge of the extent of irrigation.

The model fit always underestimates the amount of $\mathrm{CO}_{2}$ produced at the interface. The mode1-predicted fluxes of $\left[\mathrm{CO}_{2}\right.$ across the sediment water interface are compared to those calculated from the observed gradient in Table III.3. The model-predicted fluxes were calculated from:

$$
J_{\text {mod }}=-\phi D_{c} d C_{1} / d z \mid z=0
$$

and the fluxes calculated from the data were calculated from: 
Table III.3. Comparison of model-predicted fluxes $\left(J_{\text {mod }}\right)$ across the sediment water interface with those calculated from the observed gradient $\left(J_{s w 1}\right)$. The details of the calculations are described in the text. The fluxes are reported in units of $\mathrm{mol} / \mathrm{cm}^{2}-5 e c$. A negative flux is from the sediments to overlying water.

$\begin{array}{lll}\text { Month } & \underline{J}_{\text {mod }} \times 10^{11} & \underline{J}_{s w 1} \times 10^{11} \\ \text { Oct. } 83 & -0.77 & -1.21 \\ \text { June } 84 & -1.23 & -2.75 \\ \text { Aug. } 84 & -0.94 & -2.35\end{array}$




$$
J_{s w}=-\phi D_{c} \Delta C / \Delta z
$$

The model-predicted fluxes range from 40 to $65 \%$ of the observed fluxes. This indicates that the model-predicted values of $R_{c}$ underestimate the production rate at the interface and possibly overestimate the oxidation below the interface. However, the integrated amount of oxidation in the surface sediments is a reasonable estimate of the actual total oxidation.

The $\left[\mathrm{CO}_{2}\right.$ data from December 83 , March 85 , and October 85 could not be fit well with the model described above. This suggests that the sediment system was not at steady-state. If so, the above model can not be applied to the data to determine $R_{c}$. Thus, a different model will be used to estimate values of $R_{c}$ for the low temperature months.

The concentrations of $\sum \mathrm{CO}_{2}$ in December 83 and October 85 were very low compared to values observed at this site in most other months (this study; see also Martin, 1985). The December 85 core was sampled a few days after several large storms had passed through Buzzards Bay, and the October 85 core was sampled one month after Hurricane Gloria and one week after a stormy period. Both these cores appear to have had pore water constituents washed out by some mechanism and have not had time to re-establish steady-state concentrations.

In December $83^{222} \mathrm{Rn} /{ }^{226} \mathrm{Ra}$ measurements did not show depletions (Martin, 1985). In order for this system to be at equilibrium, the irrigating event must have occurred at least two weeks prior to sampling. If it is assumed that the pore water $\sum \mathrm{CO}_{2}$ between 0 to $20 \mathrm{~cm}$ were diluted to the bottom water $\mathrm{SCO}_{2}$ concentration and that it has taken two weeks to establish a constant final concentration of $2.75 \mathrm{mM}$, an estimate of the 
maximum $R_{c}$ can be calculated from:

$$
R_{c}=\Delta C / \Delta t
$$

The rate calculated for December 83 is shown in Table III.4 along with the values of $R_{c}$ calculated for October 83 , June 84 , and August 84 .

The core from March 85 is interesting and appears to be most similar to the core from June 84. A large maximum is observed in the dissolved $\mathrm{Ca}$ profile in both these months. In March 85, there is a minimum observed in the Alk profile in the upper $4 \mathrm{~cm}$, and in June 84 , the Alk measured in the surface interval was less than bottom water Alk. In both these cores there appears to be significant dissolution of $\mathrm{CaCO}_{3}$ at the same time that there is net consumption of Alk. This is particularly surprising because the dissolution of $\mathrm{CaCO}_{3}$ is a source of Alk. It is most likely that these cores were sampled at a time when benthic activity was just beginning to re-establish itself after the winter. Animals were turning the sediment over and bringing solid-phase sulfides (e.g. Fes) from depth in the sediment to the surface where they could be oxidized with $\mathrm{O}_{2}$. This process can produce a large amount of acid which can dissolve $\mathrm{CaCO}_{3}$. Based on the $\mathrm{Alk}$ and $\mathrm{Ca}$ profiles, it appears that, in June 84 , the oxidation of sulfides is important very close to the sediment water interface, while the data from March 85 indicate that the oxidation occurs deep in the sediments - between 2 and $4 \mathrm{~cm}$. This means that $\mathrm{O}_{2}$ must be introduced to the sediments to a depth of $4 \mathrm{~cm}$. Irrigation is the only mechanism that could account for this. Further evidence for oxidation of sulfides is shown by the dissolved iron measured in June 84 (Martin, 1985). The maximum $\mathrm{Fe}$ concentration of $600 \mu \mathrm{M}$ is observed at 0.25 $\mathrm{cm}$ and is one of the highest measured at this site. The oxidation of 
Table III.4. Model-calculated $\mathrm{CO}_{2}$ production rates $\left(\mathrm{R}_{\mathrm{c}}\right)$ and values calculated from measured sulfate reduction rates ( $R_{\text {meas }}$ ). The units of the rate are mol/L-sec; the values in parentheses are the rates expressed as $\mathrm{nmol} / \mathrm{cm}^{3}-\mathrm{day}$.

\begin{tabular}{|c|c|c|}
\hline Month & $\underline{R}_{c} \times 10^{9}$ & $\underline{R}_{\text {meas }} \times 10^{\circ}$ \\
\hline Oct. 83 & (294) & -- \\
\hline Dec. 83 & $(45)$ & $(77)$ \\
\hline June 84 & $4.25(312)$ & $2.7(196)$ \\
\hline Aug. 84 & $(551)$ & -- \\
\hline
\end{tabular}


solid-phase sulfides has been proposed as one mechanism for the removal of reduced sulfur from coastal sediments (Aller, 1982; Howarth, 1984). The data from these cores provide compelling evidence that this process does occur. III.2.B.3. Summary of Solution Model Results

Rates of $\mathrm{CO}_{2}$ production in the sediments for four months are listed in Table III.4. The values of $R_{c}$ calculated from this model for the surface sediment range from a low of $4.0 \times 10^{-9} \mathrm{~mol} / \mathrm{L}-\mathrm{sec}\left(294 \mathrm{nmol} / \mathrm{cm}^{3}\right.$-day) in October 83 to a high of $7.5 \times 10^{-9} \mathrm{~mol} / \mathrm{L}-\mathrm{sec}\left(551 \mathrm{nmol} / \mathrm{cm}^{3}\right.$-day) in August 84. Values of $R_{c}$ at 10 and $20 \mathrm{~cm}$ are $3-13 \%$ and $0.1-2 \%$ of the surface remineralization rates, respectively. It was found that the model-predicted value of $R_{c}$ was dependent on the value chosen for the irrigation parameter, $\alpha$. This dependence emphasizes the importance of a knowledge of the mechanisms and magnitude of pore water transport in a nearshore environment when modelling pore water profiles. At this study site the enhanced transport of solutes had been well-characterized with ${ }^{222} \mathrm{Rn}$ (Martin, 1985).

During colder months, the pore water profiles were not at steady-state concentrations. In these months, the rate of remineralization was slow and in two months, an event such as storm-induced irrigation, had reduced pore water concentrations to near bottom water values. It was possible to calculate a $\mathrm{CO}_{2}$ production term for only one of these months (December 83 ). The value of $R_{c}$ was $0.6 \times 10^{-9} \mathrm{~mol} / \mathrm{L}-\mathrm{sec}\left(45 \mathrm{nmol} / \mathrm{cm}^{3}-\right.$ day), significantly lower than those calculated in the summer months.

Table III. 4 also 1 ists $\mathrm{CO}_{2}$ production rates that were calculated from sulfate reduction rates measured in sediment from this site with ${ }^{35} \mathrm{~S}$ (Howarth, unpublished). The sulfate reduction rates were converted to $R_{c}$ 
values by assuming that two moles of $\mathrm{CO}_{2}$ are produced per mole of $\mathrm{SO}_{4}$ reduced. This relationship has been shown true in Long Island Sound (Westrich, 1985). Then:

$R_{c}=2 R_{s}$

While the measured and model-calculated rates are of the same order of magnitude, in December 83 the measured rate is almost twice the model-calculated rate, and in June 84 the measured rate is approximately one-half the model-calculated rate. The value of $R_{c}$ calculated for June 84 may be higher than the measured rate because $\mathrm{O}_{2}$, as well as $\mathrm{SO}_{4}$, may be an important electron acceptor in the surface sediments. Jorgensen et al. (19) have estimated that as much as $50 \%$ of the $\mathrm{CO}_{2}$ produced at the surface of reducing sediments overlain by an oxic water column may be from $\mathrm{O}_{2}$ reduction. If the Mn profile from June 84 (Martin, 1985) is used to define an $\mathrm{O}_{2}$ zero depth of $0.25 \mathrm{~cm}$, it can be shown that approximately $10 \%$ of the total organic carbon oxidized may be oxidized by $\mathrm{O}_{2}$. This is a minimum estimate for two reasons. First, a linear gradient was assumed for the $\mathrm{O}_{2}$ profile. Second, diffusion across the sediment water interface was assumed to be the only source of $\mathrm{O}_{2}$ to the sediments when irrigation may be a source also. In light of the above discussion, the discrepancy between the measured and model-calculated rates in December 83 is difficult to explain. Although, as mentioned before, irrigation is negligible this month and $\mathrm{O}_{2}$ is unlikely to be as important an oxidant below the sediment water interface, the relative lack of oxygen can not explain why the measured rate is higher than the calculated rate. However, some of the discrepancy may be due to spatial heterogeneity. Because the values of $R_{c}$ calculated in this study represent 
an average over a larger area of sediment than the sulfate reduction rates, $R_{c}$ is a better indicator of the average remineralization occurring at a given site.

\section{III.2.B.4. Solid-phase Organic Carbon Model}

A model was developed to interpret the solid-phase data; it is shown pictorially in Figure III.8. Basically, the model is an attempt to describe the observed profiles of SOC assuming that the major processes affecting SOC concentrations are oxidation of organic carbon and bioturbational mixing of the sediments. In the model, the sediment column is divided into two zones one with rapid mixing and another with slower mixing. The specific assumptions incorporated into the model are described below.

Mixing is assumed to be a random process in which each particle is as likely to be mixed as another. It is unlikely that this is strictly true. Benthic organisms appear to choose specific particles preferentially during their feeding and excretion activities (Jumars et al., 1981). This specificity is likely to concentrate certain particles, probably organic-rich ones, at the surface of the sediment. The sophistication necessary to include this specificity is beyond the scope of the present model and unjustified by the data set. The existence of a rapidly mixed layer in the upper few $\mathrm{cm}$ of the sediments in Buzzards Bay has been documented before. Martin (1985) showed that the short-1ived isotope ${ }^{234} \mathrm{Th}\left(t_{1 / 2}=24.1\right.$ days $)$ could be detected to depths of 2.5 to $3.0 \mathrm{~cm}$; the depth of penetration varied seasonally. Rhoads (1967) demonstrated in the laboratory that Nephthys incisa vigorously reworked sediment to depths of $2-3 \mathrm{~cm}$. The occurrence of bomb-produced radionuclides to depths of almost $30 \mathrm{~cm}$ at Station $P$ in Buzzards 
Figure III.8. Representation of the model used to interpret the solid-phase data. 
Figure III.8

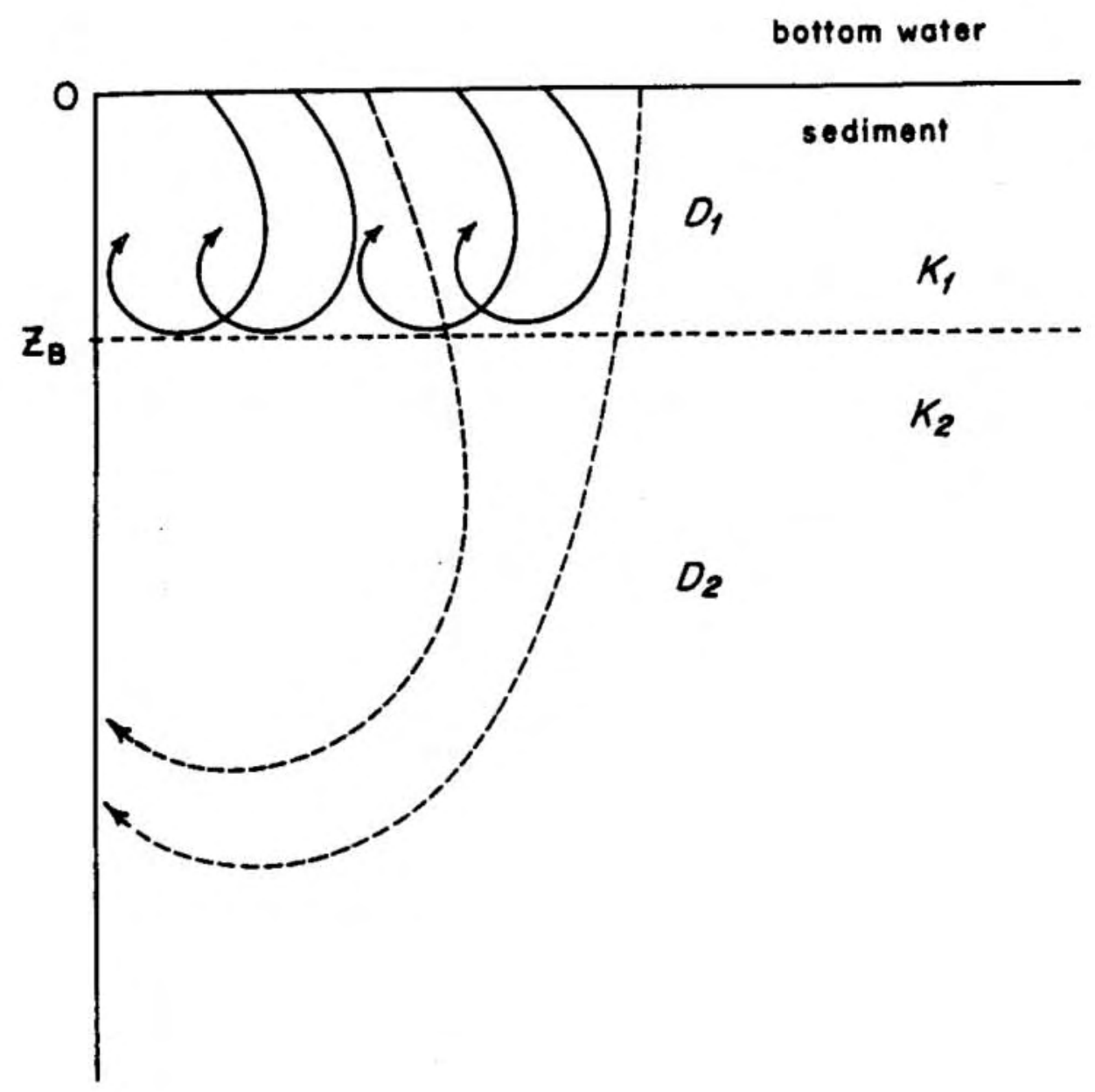


Bay (Sholkovitz and Mann, 1984) is evidence that solid-phase mixing occurs to depths much greater than $3 \mathrm{~cm}$. However, mixing in the second zone is considered to be slow enough for sedimentation to affect the depth profile of SOC. Each mixing coefficient is assumed to be constant with depth in the region in which it is defined.

The oxidation rate of organic carbon is assumed to be proportional to the concentration of metabolizable organic carbon. Westrich and Berner (1984) have shown that the decompositon of plankton can be described by a first order rate equation and that the rate of $\mathrm{SO}_{4}$ reduction in sediments is directly proportional to the quality of the organic matter added to the sediments. Two rate constants, $k_{1}$ and $k_{2}$, are defined in this model; the first applies to the oxidation of organic carbon in the rapidly mixed layer and the second to the deeper layer of sediments. Two constants are used in an attempt to account for the different reactivities of certain fractions of the organic matter. The rate constants are assumed to change at the boundary between the two mixing layers for two reasons. One is to simplify the mathematics of the problem. The other is that the depth of penetration of highly labile organic carbon is probably limited by the speed with which it can be mixed into the sediments. If there were no rapid mixing there would be very little high quality organic matter below the surface of the sediments.

It is assumed that the supply of organic carbon to the sediments has been constant over time. The sedimentation rate at this site is $0.075 \mathrm{~cm} / \mathrm{yr}$ (this study). The cores studied here are 30 to $40 \mathrm{~cm}$ long and represent approximately 500 years of sediment accumulation. It is likely that the sedimentation rate has changed over this time period. The transition from a 
wilderness to a populated area should have caused increased run-off as well as a greater supply of nutrients to the Bay. If the amount of organic carbon reaching the sediments has increased while the rate of remineralization has remained the same, this model will overestimate the amount of carbon oxidized in the sediments.

There is almost certainly a seasonality in the supply of organic carbon to the sediments that is related to plankton blooms. The effects of this seasonality will be discussed after discussing the results of the model application.

The porosity of the sediments is assumed to be constant with depth. As discussed in a previous section this is not strictly true; the porosity of the sediments is described by equation $3-2$.

Given the above assumptions, the following diagenetic equations may be written to describe the depth profile of metabolizable organic carbon concentration $(G)$ :

Layer I $\quad \partial G_{1} / \partial t=D_{1}\left(\partial^{2} G / \partial z^{2}\right)-k, G$ Layer II $\quad \partial G_{2} / \partial t=D_{2}\left(\partial^{2} G / \partial z^{2}\right)-\omega(\partial G / \partial z)-k_{2} G$ where $D_{1}, D_{2}$ are mixing rate coefficients $k_{1}, k_{2}$ are reaction rate coefficients $\omega$ is the sedimentation rate

To anaiytically solve these equations, the profile is assumed to be in steady-state. The validity of the steady-state assumption for dissolved species has been discussed in detail. However, it is unlikely that the solid-phase responds on the same time scale. The greatest mixing coefficients for Buzzards Bay are at an order of magnitude less than sediment diffusion 
coefficients. It is probable that a few months are required for the solid-phase organic carbon profiles to reach steady-state while only a few weeks are required for the solution profiles.

The boundary conditions used to solve the equations are:

$$
\begin{aligned}
z & =0, G=G_{0} \\
z & =z_{b}, G_{1}=G_{2} \quad z_{b}=\text { boundary between layers } I \text { and } I I . \\
& J_{1}=J_{11} \\
z & \rightarrow \infty, G_{11} \rightarrow 0
\end{aligned}
$$

The solutions are:

$$
\begin{aligned}
& G_{1}=C_{1} e^{\gamma z}+C_{2} e^{-y z} \\
& G_{11}=C_{4} e^{\beta z}
\end{aligned}
$$

where

$$
\begin{aligned}
& \gamma=\left(k_{1} / D_{1}\right)^{1 / 2} \\
& B=\omega / 2 D_{2}-\left(\omega^{2} / 4 D_{2}^{2}+k_{2} / D_{2}\right)^{1 / 2} \\
& C_{1}=G_{0}-C_{2} \\
& C_{2}=\frac{G_{0} e^{\gamma 2 b}\left(B D_{2}-\omega-\gamma D_{1}\right)}{e^{-\gamma 2 b}\left(\omega-\beta D_{2}-\gamma D_{1}\right)+e^{\gamma=b}\left(B D_{2}-\omega-\gamma D_{1}\right)} \\
& C_{4}=\gamma D_{1}\left(C_{2} e^{-\gamma z b}-C_{1} e^{\gamma=b}\right) / e \beta=b\left(\omega-\beta D_{2}\right)
\end{aligned}
$$

In order to use this model to determine reaction rates, the following parameters must be defined: $G, G_{0}, D_{1}, D_{2}, w, Z_{b}$

Metabolizable organic carbon was operationally defined as the concentration of organic carbon at a particular depth minus the asymptotic concentration of organic carbon, i.e.

$$
G_{z}=C_{z}-C_{\infty}, C_{\infty}=16.0 \mathrm{mgC} / \mathrm{gdw} \text { (in June } 8415.0 \mathrm{mgC} / \mathrm{gdw} \text { ) }
$$

The value of organic carbon measured in the surface sediment interval is 
assigned to $\mathrm{C}_{0}$.

The mixing coefficients, depth of the boundary between layers 1 and 2, and sedimentation rate were estimated by applying a 2-layer mixing model to $210 \mathrm{~Pb}$ data (Brownawe11, 1986) collected at the site. The mixing coefficient in the first layer was assumed to be equal to that calculated by Martin (1985) using his ${ }^{234} \mathrm{Th}$ data. The model applied to the excess ${ }^{210} \mathrm{~Pb}$ data is the same as that described above for the oxidation of organic carbon except that the decay constant $(\lambda)$ of the isotope being considered is used instead of the reaction rate constant, i.e. $\lambda=k_{1}=k_{2}$. The fit to the ${ }^{210} \mathrm{~Pb}$ activity profile is shown in Figure III.9. The ${ }^{210} \mathrm{~Pb}$ data are described well by this model. The depth to which rapid mixing occurs $\left(z_{b}\right)$ that was determined from the fit to the ${ }^{210} \mathrm{~Pb}$ data is $3 \mathrm{~cm}$. This is consistent with excess ${ }^{234} \mathrm{Th}$ data (Martin, 1985) and laboratory observations of sediment from Buzzards Bay (Rhoads, 1974). A value of $0.05 \mathrm{~cm} / \mathrm{yr}$ is used as the sedimentation rate (Section V.4). The value of $\omega$ is not critical in the analysis of the solid-phase organic carbon data because $D_{2}$ is large enough that sedimentation does not affect the calculated profile. The values of $D_{1}, D_{2}, Z_{b}$, and $\omega$ used in running the organic carbon oxidation model are defined in Table III.5.

III.2.B.5. Solid-Phase Model Results and Discussion

Model fits to the metabolizable organic carbon data are shown in Figures III. 10 a to e; the best fit is drawn with a solid line. The reaction rate constants calculated from the model are listed in Table III.7. The values of $k_{1}$ and $k_{2}$ used to fit the data are dependent on the mixing coefficients 
Figure III.9. Fit of the ${ }^{210} \mathrm{~Pb}$ data to the solid-phase mixing model. The mixing coefficients used were $D_{1}=44.0 \times 10^{-8} \mathrm{~cm}^{2} / \mathrm{sec}$ and $D_{2}=11.0 \times$ $10^{-8} \mathrm{~cm}^{2} / \mathrm{sec}$. 
Figure III.9

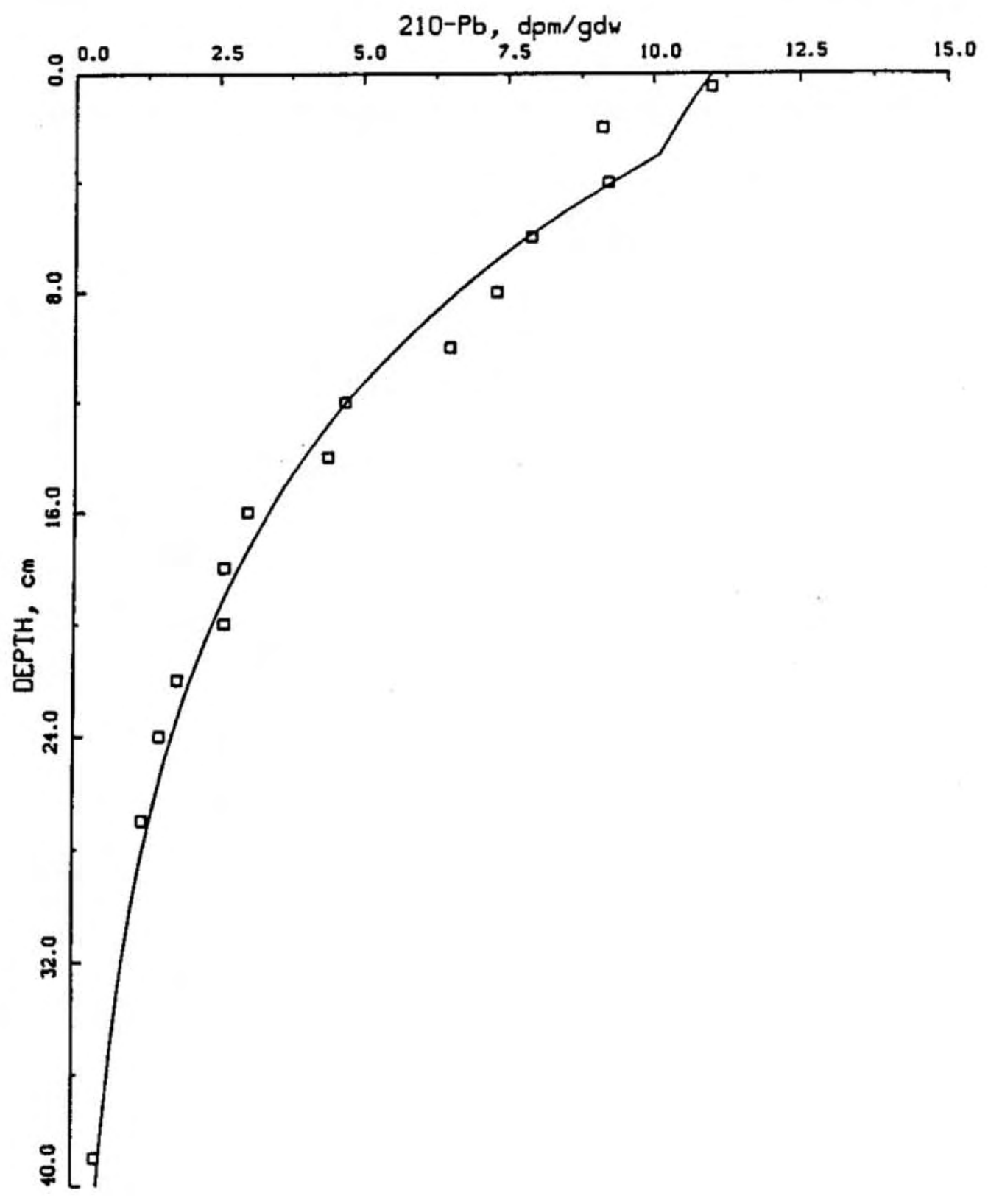


Table III.5. Model parameters used to fit solid-phase data to model. Model is described in text. $D_{1}$ and $D_{2}$ are expressed in $\mathrm{cm}^{2} / \mathrm{sec} ; z_{b}$ in $\mathrm{cm}$; and $\omega$ in $\mathrm{cm} / \mathrm{sec}$.

Month

$\underline{D_{1}}$

$\underline{D}_{2}$

$\underline{z}_{b}$

$\underline{\omega}$

Oct. 83

June 84

$44 \times 10^{-8}$

$11 \times 10^{-8}$

3

$1.59 \times 10^{-9}$

Aug. 84

Dec. 83

$22 \times 10^{-8}$

$5.5 \times 10^{-8}$

3

$1.59 \times 10^{-9}$

Oct. 85 
Figure III.10. Fits of the solid-phase model to the metabolizable organic carbon data. The parameters used to fit the data are listed in Tables III.5 and III.6. The solid lines represent the best fits; the dotted lines were fit using values of $k_{1}$ and $k_{2}$ five times the best-fit values, and the dashed lines were calculated using values of $k_{1}$ and $k_{2}$ one-fifth the best-fit values. 

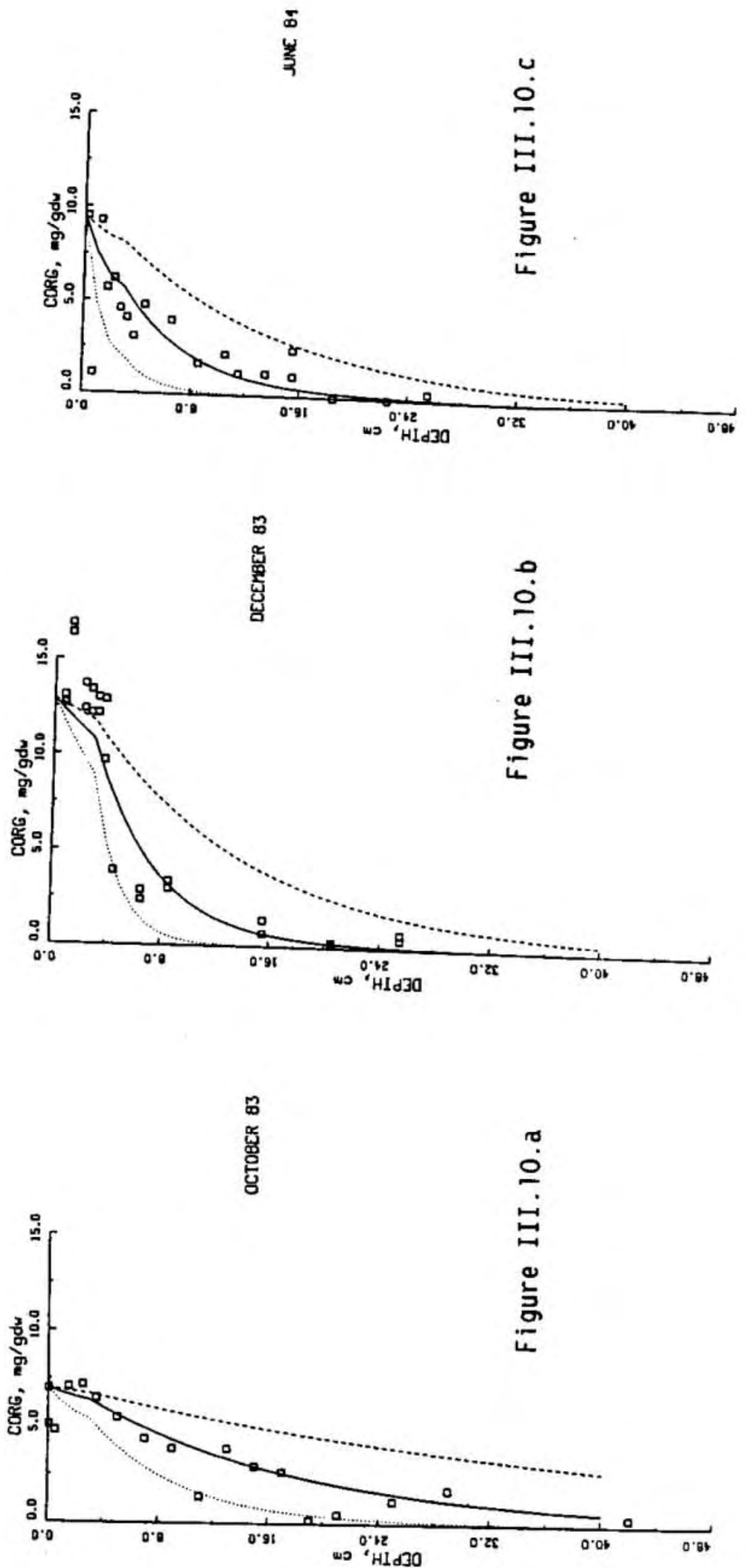

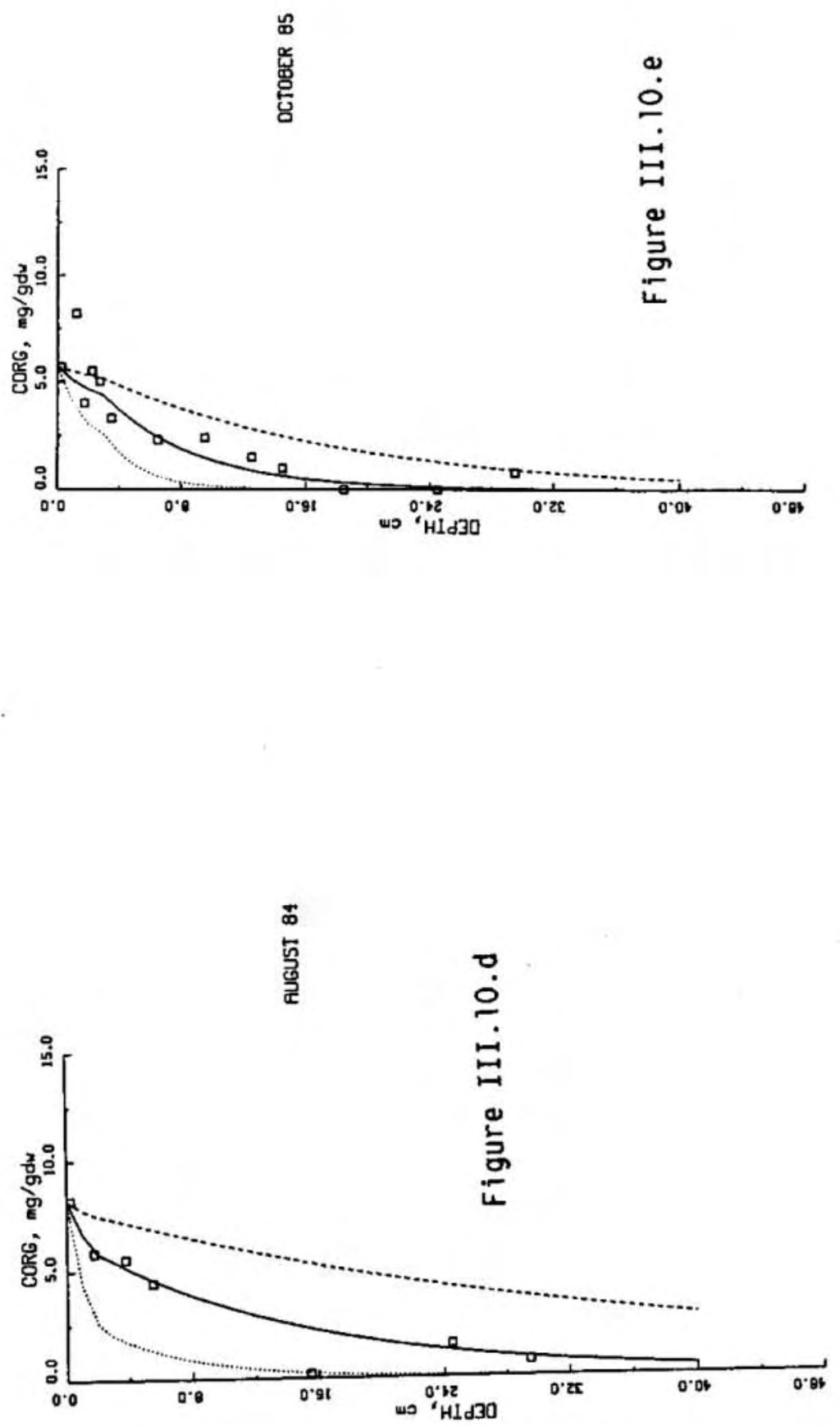
Table III.6. Values of the reaction rate constants $\left(k_{1}\right.$ and $\left.k_{2}\right)$ calculated for each month. The constants are expressed as $\mathrm{sec}^{-1}$.

Month $\quad \underline{k}_{1} \times 10^{9} \quad \underline{k}_{2} \times 10^{9}$

Oct. 83

3.0

0.6

Dec. 83

0.5

3.0

June 84

44.0

5.0

Aug. 84

40.0

0.7

oct. 85

7.3

1.8 
used. However, there is enough variability in the organic carbon data that changing the value of $D_{1}$ or 2 by a factor of two does not greatly alter the value of $k_{1}$ or 2 .

The reaction rate constants calculated in the upper $3 \mathrm{~cm}$ vary a great deal seasonally, while those calculated from data collected deeper in the sediment column are approximately equal. The variability in the surface sediments probably reflects the inadequacy of two of the assumptions in the model. The system is not at steady-state and the estimation of metabolizable organic carbon is a gross simplification of the complexity of organic matter in the sediments. It is also possible that the calculated variations are actually due to temporal variations. Possible explanations of the variable rates in the surface sediments are related to seasonal fluctuations in temperature, the size and location of the microbial population, and the addition of highly labile organic matter.

It is likely that there is periodic addition of highly labile organic matter to the surface sediments, particulariy in the warmer months. It is possible to estimate the amount of "fresh" organic matter that must be added to the sediments to account for the elevated rates observed in June and August, ' 84 . It can be assumed that there is a constant flux of bulk organic matter with a reactivity on the order of $k_{2}$ to the sediment water interface and, occasionally, fresh, highly reactive organic matter is added, i.e.

$$
\begin{aligned}
& G=G_{b u 1 k}+G_{\text {fresh }} \\
& k_{1} G=k_{2} G_{b u 1 k}+k_{f r e s h} G_{f r e s h}
\end{aligned}
$$

The amount of fresh organic matter that must be added to the sediments to raise $k_{2}$ to $k_{1}$ can be calculated if a reaction rate constant for the fresh 
organic matter can be found. If the constant determined by Westrich and Berner (1984) in a laboratory study of the decomposition of fresh phytoplankton $\left(k=760 \times 10^{-9} \mathrm{sec}^{-1}\right)$ is used, then only $5-10 \%$ of the metabolizable organic carbon in June 84 and August 84 is from a fresh "planktonic" source. This is a lower limit of the amount of carbon necessary to raise $k_{2}$ to $k_{1}$ because the reaction rate constant determined by Westrich and Berner (1984) is an upper limit of the reactivity of the organic matter that could be expected to reach the sediment water interface. It is conceivable that, even if the reactivity of the fresh source of organic carbon were less than assumed, there could be a transient event which increases the concentration of organic carbon in the sediments for a short period of time. The input could coincide with a plankton bloom.

\section{III.2.C. Carbon Budget}

The amount of carbon oxidized in the sediments annually can be calculated from the values of $R_{c}$ and $k$ listed in Tables III.4 and III.7. For the solution model, the amount of $\mathrm{SCO}_{2}$ produced in the upper $20 \mathrm{~cm}$ of the sediments can be calculated from:

$$
\begin{aligned}
& \sum C_{p}=F\left[\int_{0}^{z b} \phi R_{c} d z+\int_{z b}^{20} \phi R_{c} e^{-}: d z\right] \\
& \text { where } F=\text { concentration conversion factor }
\end{aligned}
$$

In the months during which the dissolution of $\mathrm{CaCO}_{3}$ is important, its effect was removed in the following manner. A curve-fitting routine was used to calculate a polynomial function that describes the concentration of $\mathrm{Ca}$ with depth. If it is assumed that the $\mathrm{Ca}$ profile is in steady-state, the net amount of $\mathrm{CO}_{3}^{2-}$ added due to dissolution can be calculated from:

$$
\int_{z 1}^{z 2}\left[D^{\prime} c_{a} d^{2} C a / d z^{2}-\alpha\left(C a-C a_{0}\right)\right]=\int_{z}^{22}-R_{c a}=\sum C_{c a}
$$


Under a steady-state assumption, the sediment diffusion coefficient for $\mathrm{Ca}$ needs to be corrected for adsorption only if the solid-phase mixing coefficient is of the same order as the adsorption corrected sediment diffusion coefficient (Berner, 1980). In June 84 , this is the case in the top 2-3 cm. The corrected diffusion coefficient is:

$$
\begin{aligned}
& D_{c a}^{\prime}=(1+K) D_{1}+D_{s}=5.4 \times 10^{-6} \mathrm{~cm}^{2} / \mathrm{sec} \\
& \text { where } \quad D_{1} \text { is the solid-phase mixing coefficient in the surface } \\
& \text { sediments } \\
& \quad K \text { is the dimensioniess adsorption constant, } 1.7 \text { from Li } \\
& \text { and Gregory (1974) }
\end{aligned}
$$

In Section III.2.A.1.b, it was suggested that the low values of $\mathrm{pH}$ observed in the pore water between 0 and $4 \mathrm{~cm}$ in June 84 were due to excess acid produced during the oxidation of solid-phase sulfides. It is probable that some of the excess acid was produced during the oxidation of organic carbon with $\mathrm{O}_{2}$. The relative changes in $\mathrm{CCO}_{2}$ and $\mathrm{Ca}$ can be used to show that organic carbon oxidation alone cannot produce enough acid. Using the relationships shown in Table $\mathrm{I} .1$ and assuming that all the $\mathrm{CO}_{2}$ produced reacts with $\mathrm{CaCO}_{3}$, it can be shown that, during aerobic oxidation of organic matter with Redfield stoichiometry,

$$
\Delta \mathrm{Ca} / \Delta \sum \mathrm{CO}_{2}=0.54
$$

Comparing the relative rates of production of dissolved $\mathrm{Ca}$ and $\mathrm{ZCO}_{2}$ between 0 and $2 \mathrm{~cm}$ in June 84

$$
R_{\mathrm{ca}} / R_{\mathrm{c}}=1.06
$$

This value is almost twice the predicted value indicating that there is a significant source of acid other than the aerobic oxidation of organic 
carbon. The fact that the measured values of pH are all less than 6.5 suggest that there is not enough $\mathrm{CaCO}_{3}$ in the sediments to neutralize all the acid produced. The fact that intact shell fragments are found deeper in the sediment indicates that there is still $\mathrm{CaCO}_{3}$ in the sediments. However, these fragments are always found at depth, usually $20 \mathrm{~cm}$ or deeper, and may have passed through the zone of dissolution too rapidly to be dissolved entirely. If it is assumed that the dissolution of $\mathrm{CaCO}_{3}$ in June represents a maximum amount of dissolution and that the same amount of dissolution can occur as many as three months of the year, then, a maximum amount of annual $\mathrm{CaCO}_{3}$ dissolution of $4.2 \mathrm{~g} \mathrm{CaCO}_{3} / \mathrm{m}^{2}$ is calculated.

Returning to the carbon budget, the amount of carbon oxidized in the sediments is then:

$$
\sum \mathrm{C}_{0 \times}=\sum \mathrm{C}_{\mathrm{p}}-\sum \mathrm{C}_{\mathrm{ca}}
$$

The amount of carbon oxidized has been calculated on an annual basis and is listed in Table III.8.

To calculate the amount of carbon oxidized in the sediments using the solid-phase model:

$$
\begin{aligned}
& \int C_{a x}=F\left[\int_{0}^{z b} k_{1} G d z+\int_{z b}^{\infty} k_{2} G d z\right] \\
& \text { where } F=\text { concentration conversion factor }
\end{aligned}
$$

The results of this calculation are listed in Table III.7.

The solution and solid-phase model results are of the same order of magnitude, but the agreement in October 83 and December 83 is not good. It is likely that a steady-state assumption is particularly bad for the solid-phase data from December 83 . The elevated values of SOC measured in the upper $4 \mathrm{~cm}$ (Figure III.1.b) probably reflect a very recent addition of fresh carbon that 
Table III.7. The calculated amount of carbon oxidized annually in Buzzards Bay sediments. The total carbon oxidized is shown for solution results $\left(\sum C_{s o 1 n}\right)$ and for solid-phase results $\left(\sum C_{s p}\right)$, and is expressed in $\mathrm{gC} / \mathrm{m}^{2}-\mathrm{yr}$.

Month $\sum \underline{C}_{\text {soin }} \quad \sum \underline{C}_{s p}$

Oct. 83 82 15

Dec. 83 19 86

June 84

89

132

Aug. 84

177

100

oct. 85

$--$

19 
has not been in the sediment long enough to be degraded. As discussed in Section III.2A.2.a, it is more likely that the solute data represent steady-state conditions. Thus, it is likely that the results calculated from the solution data are more accurate.

In Figure III.11, plots of bottom water temperature, $R_{c}, k_{1}$, and $\sum C_{o x}$ vs. time are shown. As expected there is a correlation between remineralization rate and temperature. Assuming that remineralization in October 84 returns to a rate similar to that in October 83 , the area under the $\sum C_{o x}$ curve represents an annual average of the carbon oxidized in the sediments. The amount of carbon oxidtzed to $\mathrm{CO}_{2}$ in one year in the sediments is then $85 \mathrm{~g} \mathrm{C} / \mathrm{m}^{2}$. When this is added to the amount preserved in the sediments, a total of $90 \mathrm{~g} \mathrm{C} / \mathrm{m}^{2}$ reaches the sediments. This compares reasonably well with the amount of primary productivity reported for Buzzards Bay by Roman and Tenore (1978); they estimated that there are $100 \mathrm{~g} \mathrm{C} / \mathrm{m}^{2}$ fixed annually. However, this agreement would suggest that remineralization in the water column is negligible, or that there is some mechanism which concentrates organic-rich particles in certain sediment regions. It is probable that there is such a mechanism; however, it is also possible that the actual amount of primary productivity is different from that reported by Roman and Tenore (1978). Studies of the methods used to measure primary productivity (Peterson, 1980; Goldman, 1980) suggest that older methods may underestimate the primary productivity by as much as ten times. For this discussion, it will be assumed that the productivity has been underestimated by a factor of two, thus making the estimate $200 \mathrm{gC} / \mathrm{m}^{2}-\mathrm{yr}$. the actua 1 amount of primary productivity in Buzzards Bay is an important parameter that 
Figure III.II. The relation of bottom water temperature $\left({ }^{\circ} \mathrm{C}\right), R_{c}(m o l / L-s e c$ $x\left(0^{9}\right), k_{1}\left(\mathrm{sec}^{-1} \times 10^{9}\right)$, and $\sum \mathrm{CO}_{20 \times}\left(\mathrm{gC} / \mathrm{m}^{2}-\mathrm{yr}\right)$ to the time of the year. 
Figure III.11
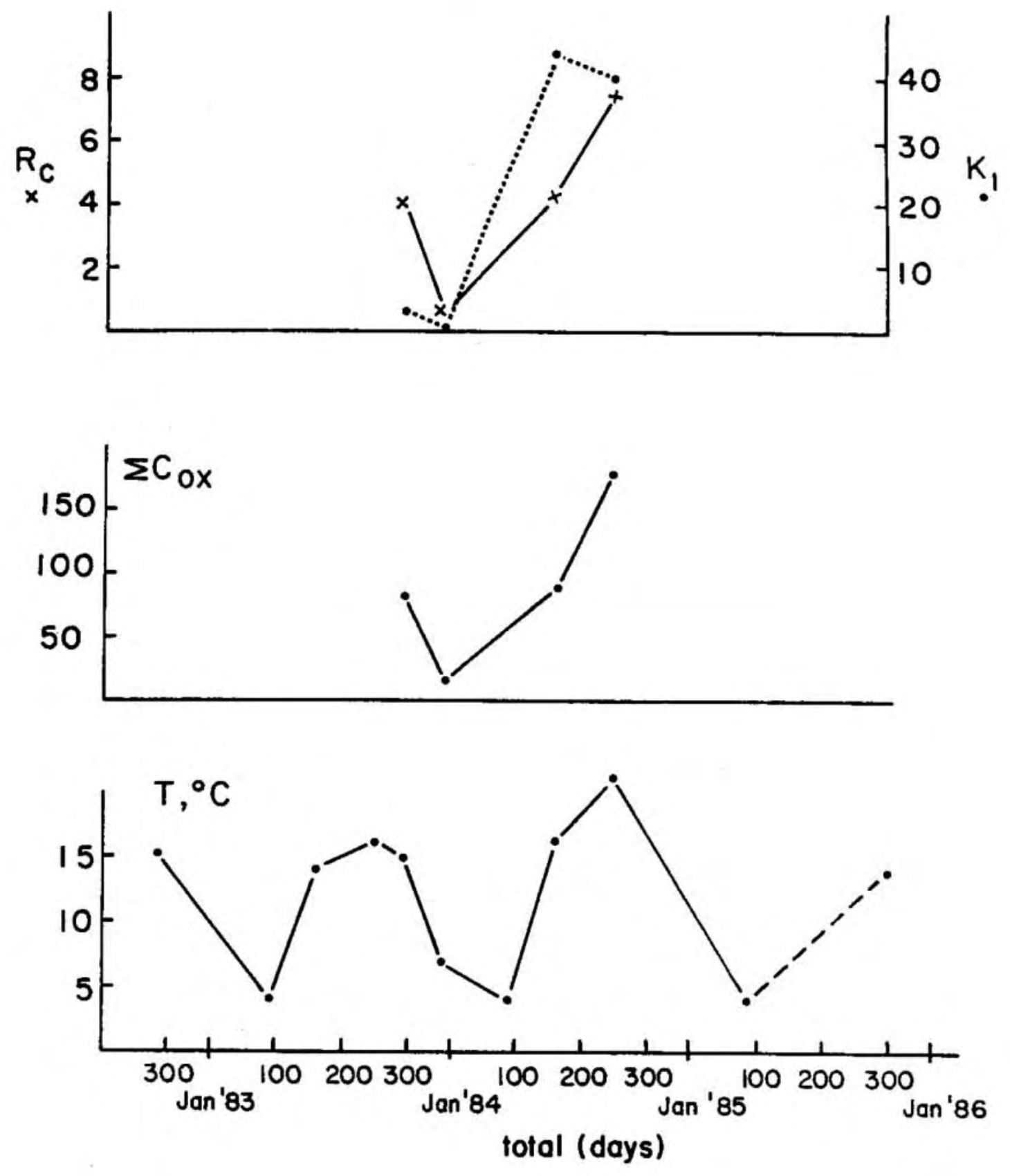
needs to be investigated. The organic carbon that reaches the sediments is concentrated in areas of fine-grained sediments and the primary productivity can be corrected to reflect this:

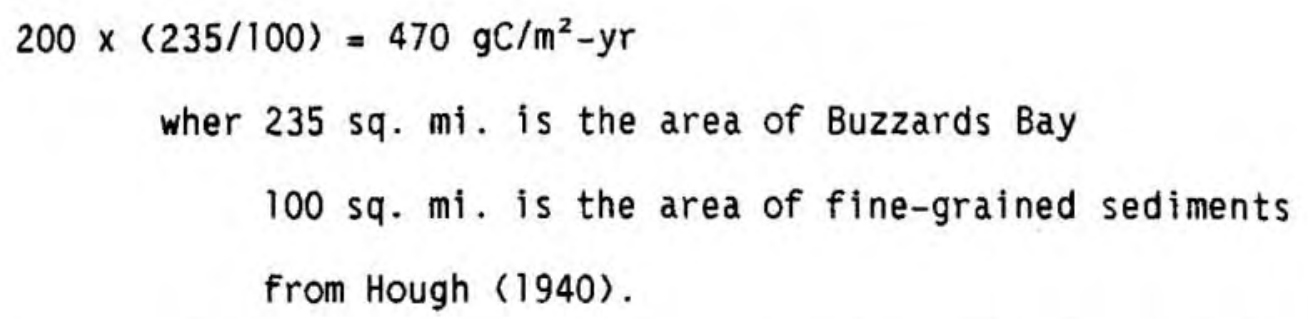

With these revisions, it is calculated that only $20 \%$ of the carbon fixed annually reaches the sediments. This value may be an overestimate because an additional component of the flux of $\mathrm{SCO}_{2}$ has not been considered. It is possible that some of the $\left[\mathrm{CO}_{2}\right.$ flux from the sediments to the bottom water originated in the bottom water. Because the $\mathrm{pH}$ of bottom water is greater than that of surface pore water, $\mathrm{CO}_{3}^{2-}$ may diffuse from the bottom water to the pore water, become protonated, and then diffuse back to the bottom water. The significance of this process will be discussed in the next chapter. 
Chapter IV

THE NATURE OF THE ORGANIC MATTER UNDERGOING OXIDATION

AND OTHER FACTORS AFFECTING THE $\delta^{13} \underline{\mathrm{C} \text { OF }} \mathrm{ICO}_{2}$

\section{IV.1. Introduction}

The oxidation of organic carbon in sediments can be traced through changes in the $\delta^{13} \mathrm{C}$ of $\left[\mathrm{CO}_{2}\right.$ in pore waters. This is a particularly useful approach as it is difficult to investigate remineralization of organic carbon in coastal sediments directly. Particulate matter in the water column is not always representative of new material reaching the sediment water interface since tidal currents, storms, and benthic activity resuspend sediments; the resuspended, fine-grained material is mixed throughout the relatively short water column. Thus, the nature, as well as the quantity, of the organic matter arriving at the sediment water interface is not always simply related to organic matter in the water column. Also, mixing is so rapid in the zone of rapid remineralization that changes in the quantity and quality of solid-phase organic carbon are difficult to measure. The production of $\mathrm{CO}_{2}$ during the oxidation of organic matter can be measured in pore waters. 
Changes in $\mathrm{CCO}_{2}$ serve as a sensitive indicator of diagenetic processes. The $\delta^{13} \mathrm{C}$ of $\left[\mathrm{CO}_{2}\right.$ must reflect the isotopic nature of the organic matter being oxidized.

In this chapter, the isotopic nature of the labile organic carbon is investigated by studying the $\delta^{13} \mathrm{C}$ of $\sum \mathrm{CO}_{2}$ in pore waters. Isotope results from a field study will be analyzed, and a laboratory study using sediments from the same site as the field study will be presented. The study site and methods used were described in Chapter II.

\section{IV.2. Results}

\section{IV.2.A. Field Study}

Depth profiles of $\delta^{13} \mathrm{C}-\sum \mathrm{CO}_{2}$ from cores collected between October 83 and October 85 were shown in Figure III. 3 a to $f$; the data are listed in Table AI of Appendix I. In general, there is a decrease in $\delta^{13} \mathrm{C}$ with depth in the sediments. Bottom water values average about $0.00 / 00$ and by 30 $\mathrm{cm}$ the $\delta^{13} \mathrm{C}-\left[\mathrm{CO}_{2}\right.$ is approximately $-10.0 \%$ o 0 . In October 83 and June 84 , there is a minimum in the $\delta^{13} \mathrm{C}$ profile between 4 and $10 \mathrm{~cm}$. The relationship of $\delta^{13} \mathrm{C}-\sum \mathrm{CO}_{2}$ to $\sum \mathrm{CO}_{2}$ for each month is shown in Figure IV.1 a to $f$, and for the combined data in Figure IV.2. In most months, a negative correlation is apparent. The lines that fit the data best for each month are shown in Figure IV.I a to $f$ and listed in Table IV.l along with the correlation coefficients. The data from June 84 are described best by two lines because the data collected between 0 and $4 \mathrm{~cm}$ appear anomalously enriched in ${ }^{13} \mathrm{C}$. This anomaly will be discussed in Section IV.3.E. These data were not used to calculate the best fit line for the combined data. 
Figure IV.1. Measured values of $\delta^{13} \mathrm{C}-\sum \mathrm{CO}_{2}$ plotted against $\sum \mathrm{CO}_{2}$ for each month sampled. 


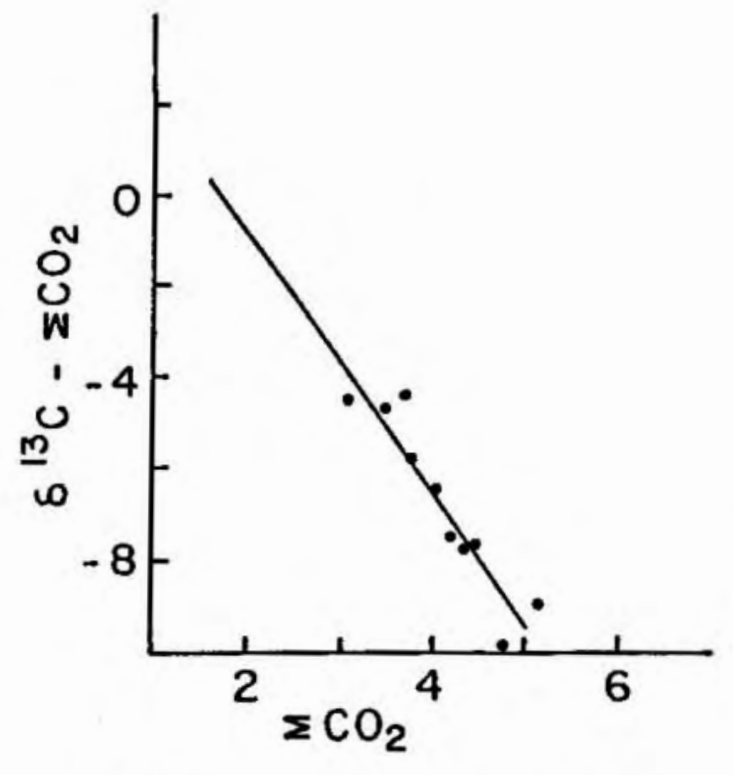

Figure IV.1.a. October 83

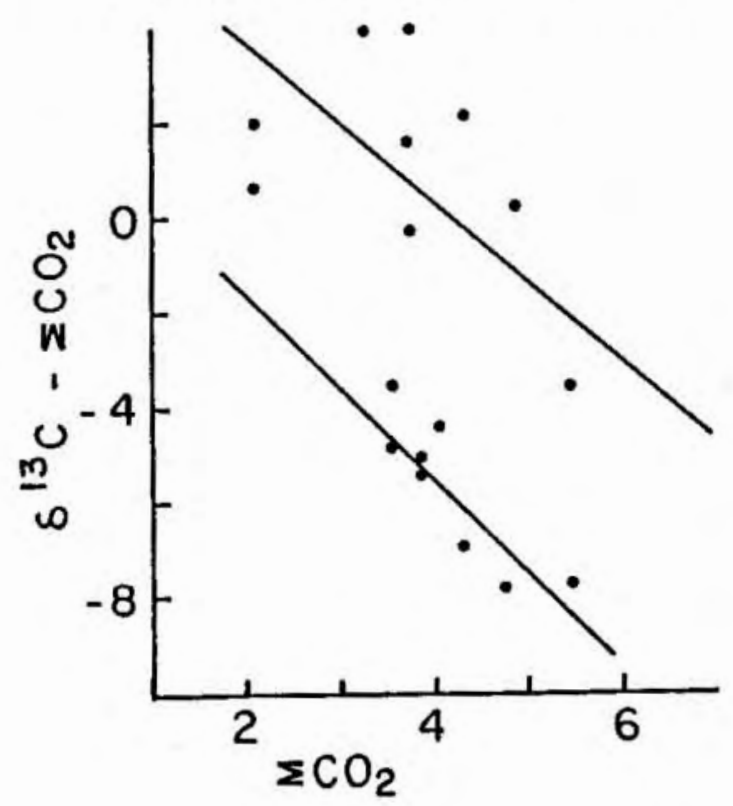

Figure IV.1.C. June 84

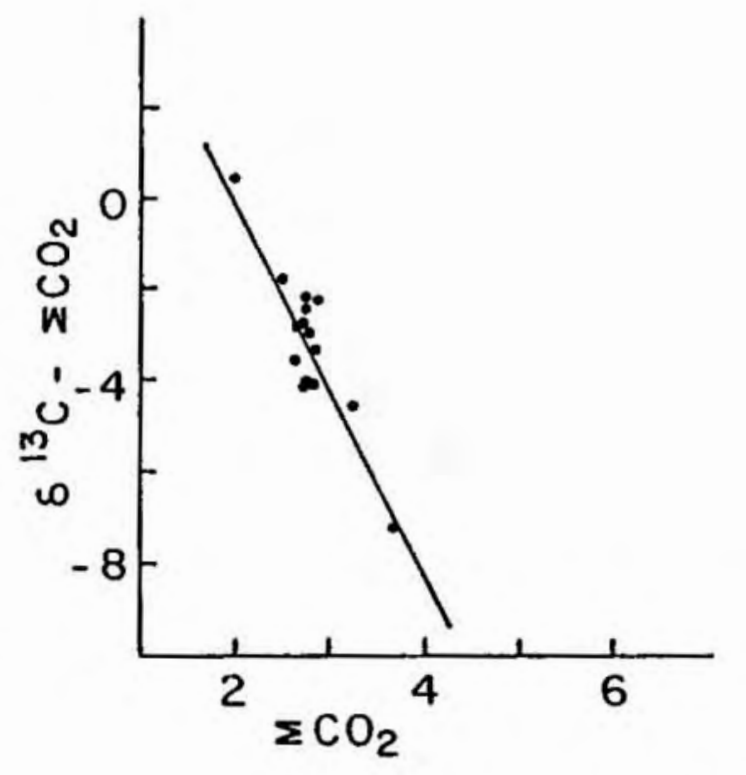

Figure IV.1.b. December 83

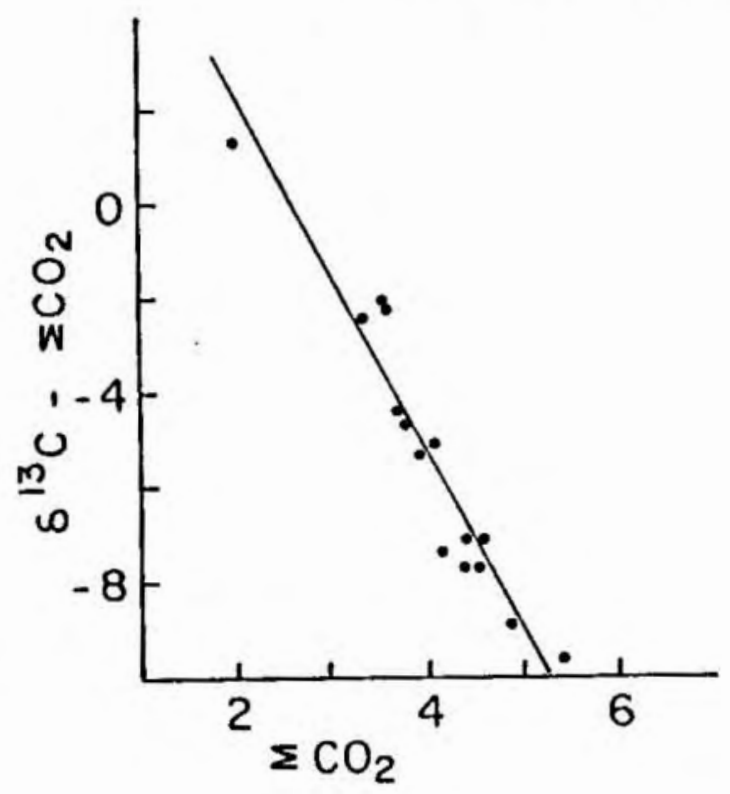

Figure IV.1.d. August 84 

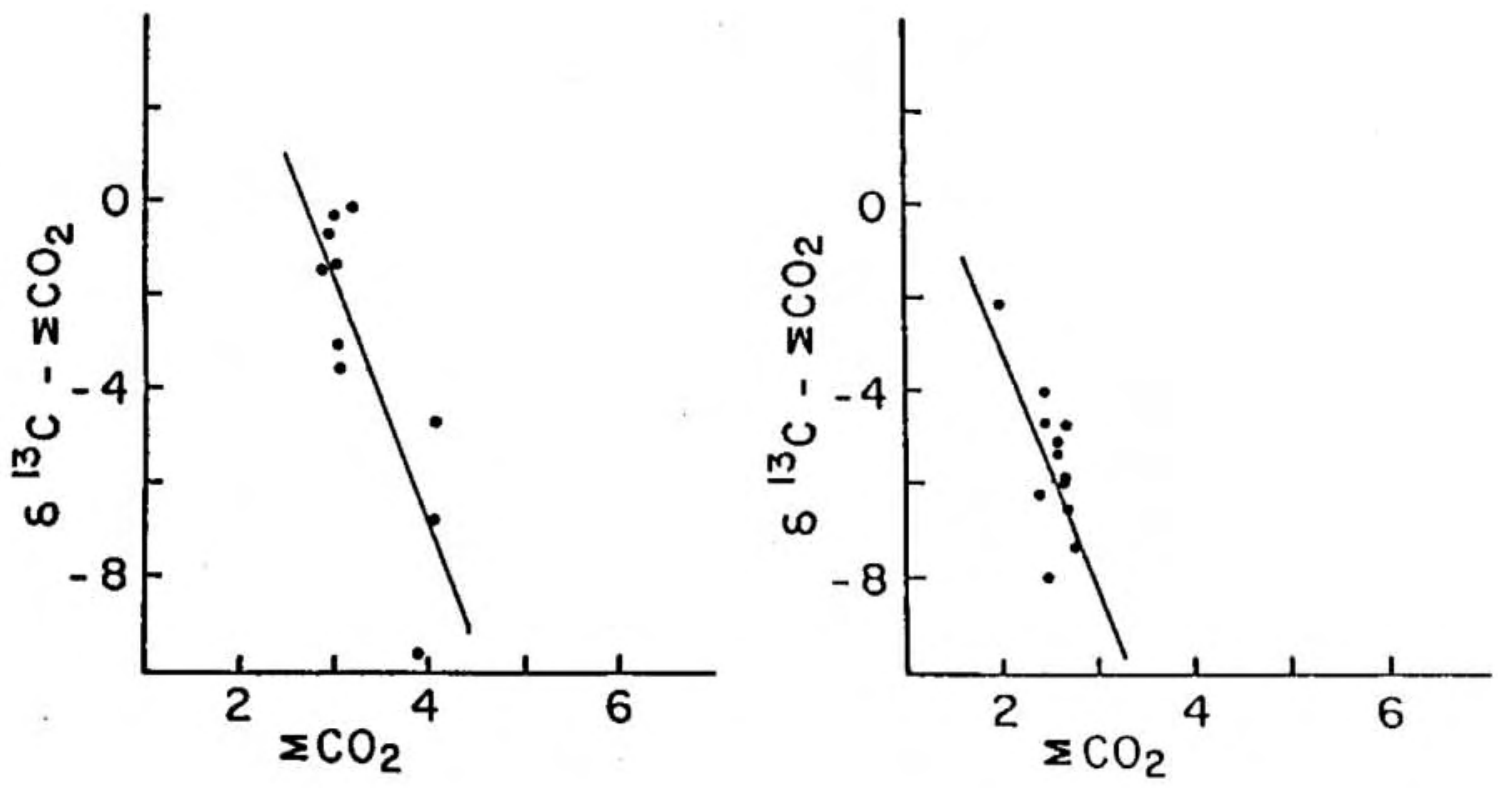

Figure IV.1.e. March 85

Figure IV.1.f. October 85 
Figure IV.2. Measured values of $\delta^{13} \mathrm{C}-\left[\mathrm{CO}_{2}\right.$ for all the months

sampled. The line shown in the plot represents the best fit calculated using data from October 83 to March 85 and excluding data between 0 and $4 \mathrm{~cm}$ from June 84 . 
Figure IV.2

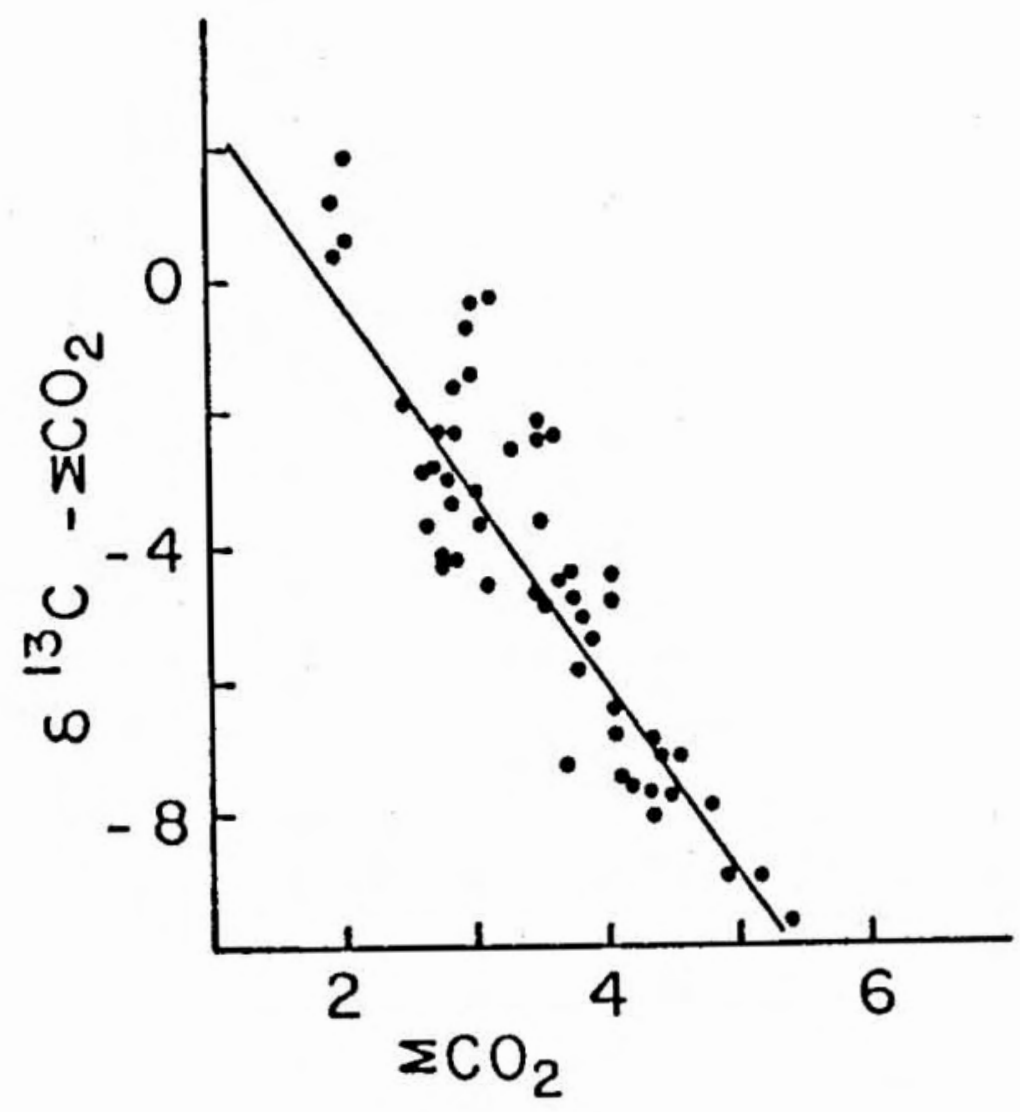


Table IV.1. Calculated best-fit lines for $\delta^{13} \mathrm{C}$ vs. $\left[\mathrm{CO}_{2}\right.$ for each month and for all the data. The lines calculated for all the data together were calculated excluding the data from June 84 between 0 and $4 \mathrm{~cm}$. These data are explained in Section IV.3.E.

October 83

$$
\begin{gathered}
\delta^{13} \mathrm{C}=-2.84\left[\mathrm{CO}_{2}+4.89\right. \\
r^{2}=.86
\end{gathered}
$$

December 83

$$
\begin{gathered}
\delta^{13} \mathrm{C}=-4.07 \sum \mathrm{CO}_{2}+8.08 \\
r^{2}=.79
\end{gathered}
$$

June 84

$$
\begin{array}{cc}
0-4 \mathrm{~cm} & \delta^{13} \mathrm{C}=-1.64\left[\mathrm{CO}_{2}+6.94\right. \\
r^{2}=.49 & \\
4-26 \mathrm{~cm} & \delta^{13} \mathrm{C}=-1.96\left[\mathrm{CO}_{2}+2.30\right. \\
r^{2}=.63
\end{array}
$$

August 84

$$
\begin{gathered}
\delta^{13} \mathrm{C}=-3.74\left[\mathrm{CO}_{2}+9.76\right. \\
r^{2}=.91
\end{gathered}
$$

March 85

$$
\begin{gathered}
\delta^{13} \mathrm{C}=-5.31 \sum \mathrm{CO}_{2}+14.30 \\
r^{2}=.67
\end{gathered}
$$

October 85

$$
\begin{gathered}
\delta^{13} \mathrm{C}=-5.08 \mathrm{CCO}_{2}+7.29 \\
r^{2}=.44
\end{gathered}
$$

October 83-

March 85

$$
\begin{gathered}
\delta^{13} \mathrm{C}=-2.86\left[\mathrm{CO}_{2}+5.58\right. \\
r^{2}=.74
\end{gathered}
$$


The bottom water and surface sediment data from October 85 appear to be very light with respect to the other months. Since a new stripping line was used to collect these data, it is possible that there was an analytical problem in stripping the $\delta^{13} \mathrm{C}-\left[\mathrm{CO}_{2}\right.$ samples this month. The data from October 85 will not be used in the calculations in this chapter.

IV.2.B Incubation Studies

Two laboratory studies of the oxidation of organic carbon in Buzzards Bay sediments were conducted; the detailed methods involved in each experiment were described in Chapter II. Briefly, in each experiment, sediment from three different depth horizons was collected and transferred to 40 to $50 \mathrm{ml}$ glass tubes. Sediment for Incubation Experiment I (IEI) was collected in February 1985 and for Incubation Experiment II (IE2) in August 1985. In IEl, the sediment from each depth horizon was stirred before transferring to the tubes. IE2 was conducted because it appeared that IEI had not reproduced the conditions found in the field. Therefore, in IE2, mud was subcored directly from diver-collected cores into the incubation tubes. Evidence that the method used for IE2 was a much less disruptive sediment collection method, and more accurately portrays field conditions will be presented in Section IV.2.B.1.

The changes of $\delta^{13} \mathrm{C}-\left[\mathrm{CO}_{2}, \sum \mathrm{CO}_{2}\right.$, and Alk with time for both incubation experiments are shown in Figure IV. $3 \mathrm{a}$ and $\mathrm{b}$ and listed in Appendix I. Also listed in Appendix $\mathrm{I}$ are the measured values of $\mathrm{H}_{2} \mathrm{~S}$ and $\mathrm{PO}_{4}$. In both experiments and at all depths, $\delta^{13} \mathrm{C}-\sum \mathrm{CO}_{2}$ decreases and $\mathrm{ZCO}_{2}$ and Alk increase over time. In both experiments, the rate of change of $\delta^{13} \mathrm{C}-\sum \mathrm{CO}_{2}, \sum \mathrm{CO}_{2}$, and $\mathrm{Alk}$ was much greater in the surface sediments 
Figure IV.3. The measured values of $\delta^{13} \mathrm{C}-\left[\mathrm{CO}_{2}(0 / 00), \sum \mathrm{CO}_{2}(\mathrm{mM})\right.$, and AIk (meq/1) for IEI and IE2 are plotted against time (days). 
Figure IV.3.a.

IE1, 0-2 cm
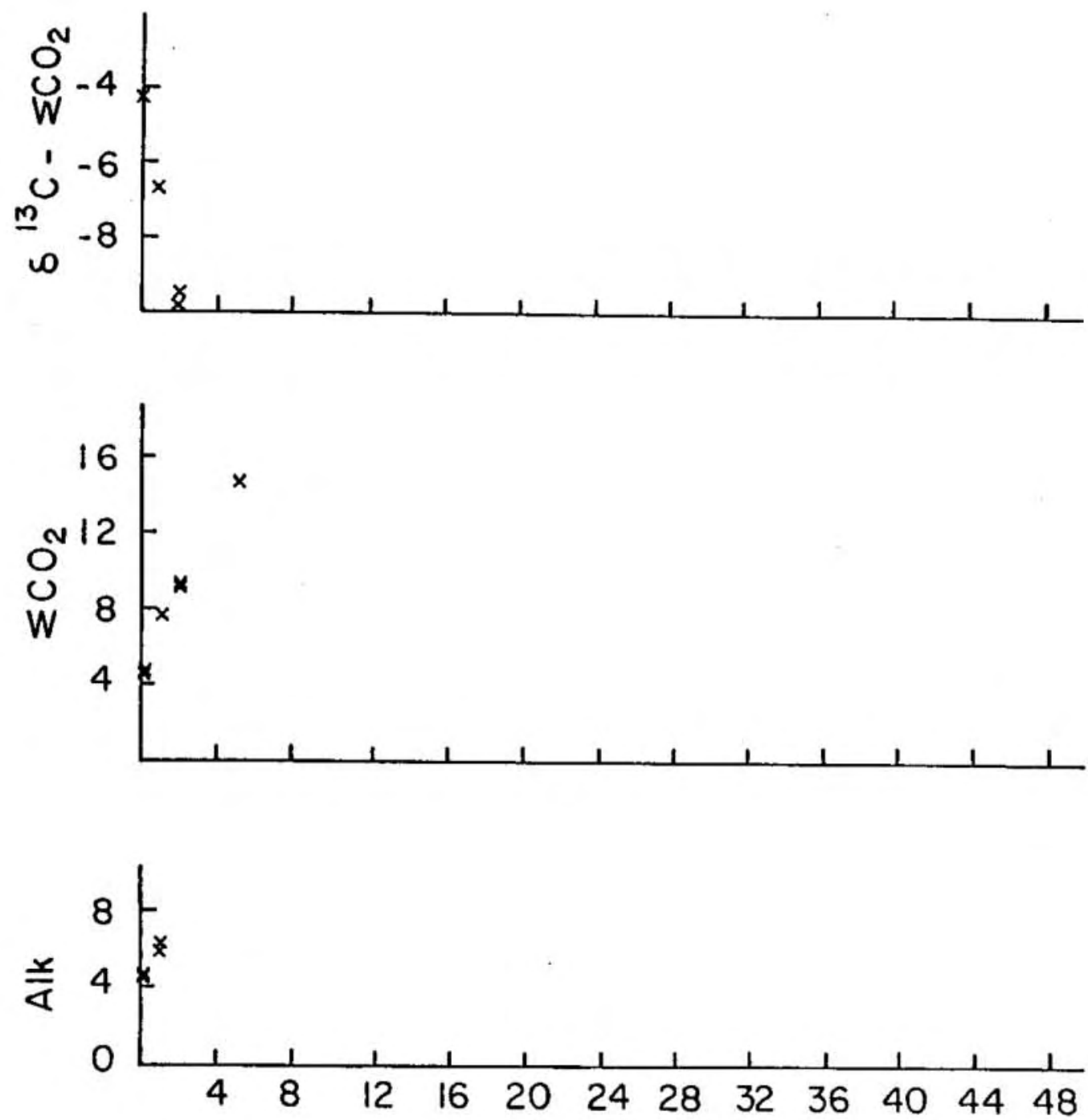
Figure IV.3.b.

IEl, 9-11 cm
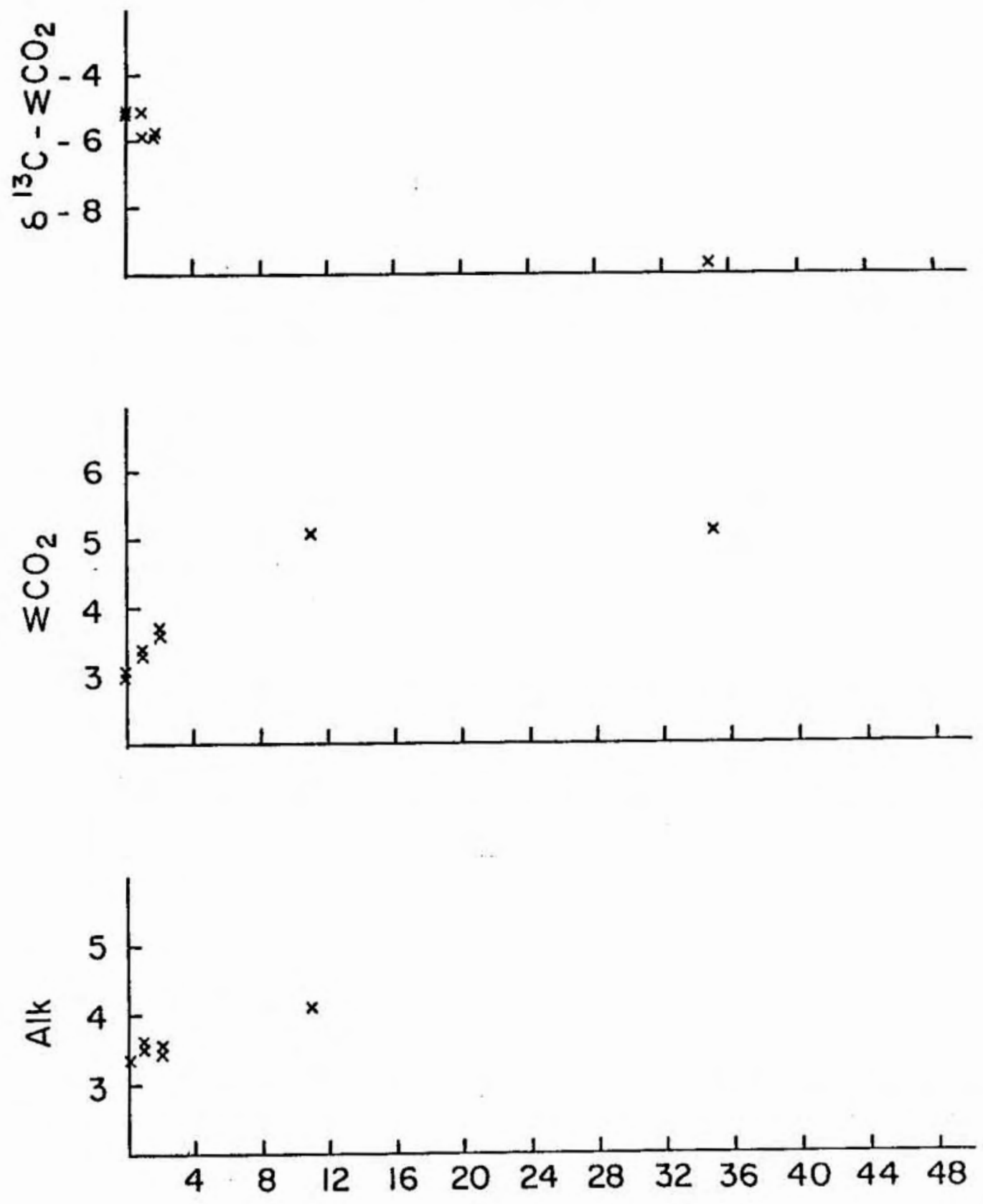
Figure IV.3.c

IEl, $17-20 \mathrm{~cm}$
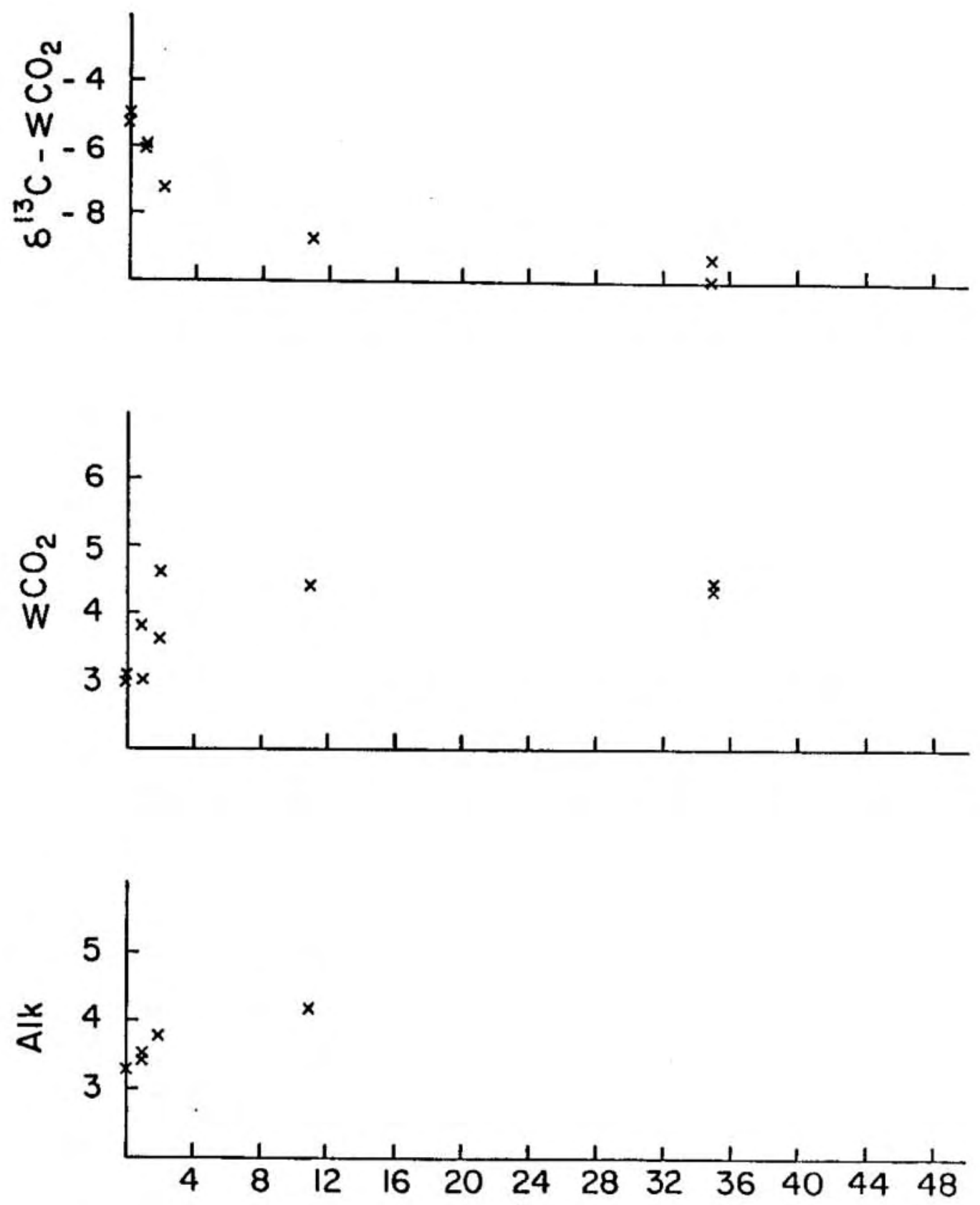
Figure IV.3.d

IE2, $0-3 \mathrm{~cm}$
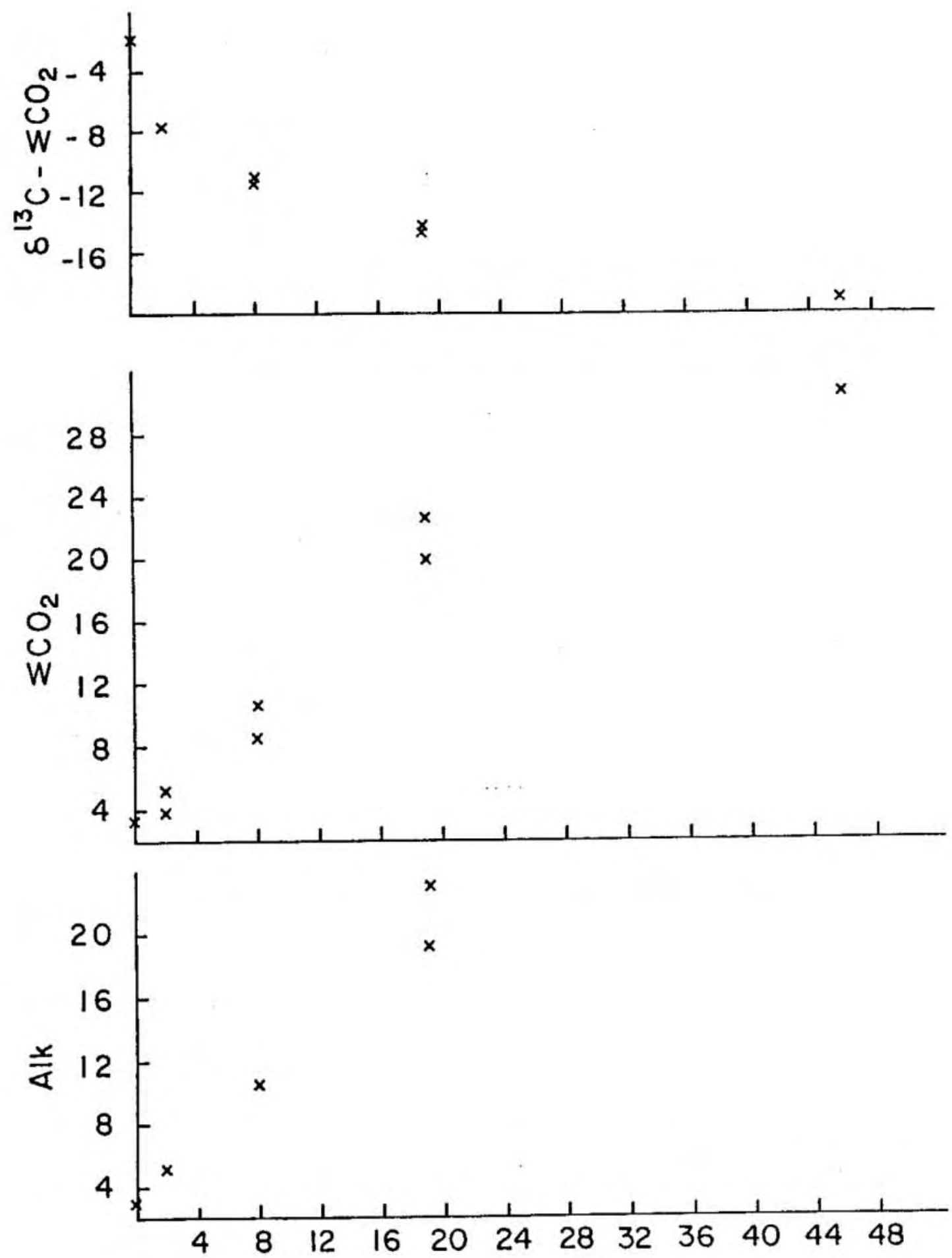
Figure IV.3.e

IE2, 12-15 cm
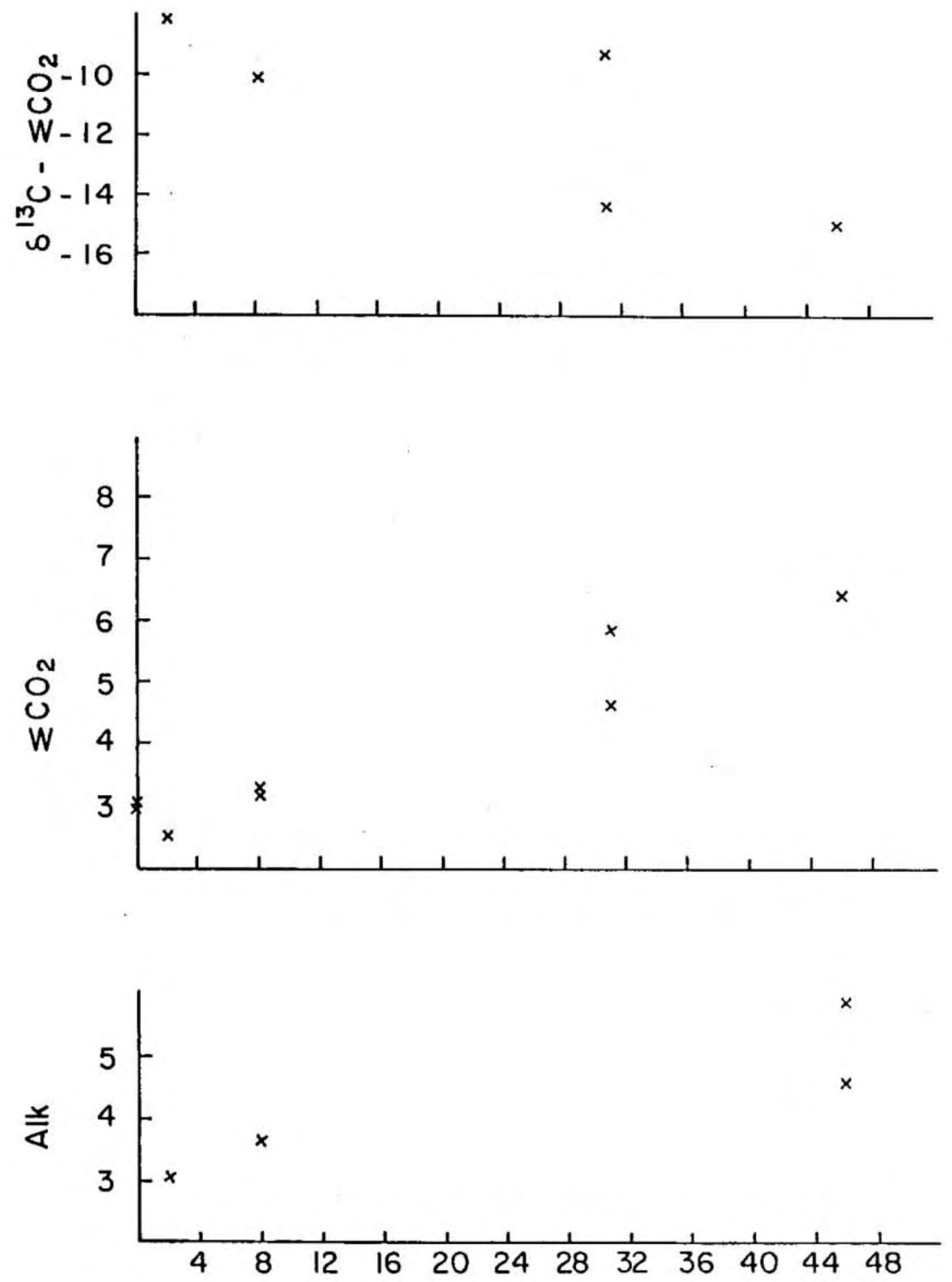
Figure IV.3.f

IE2, $18-21 \mathrm{~cm}$
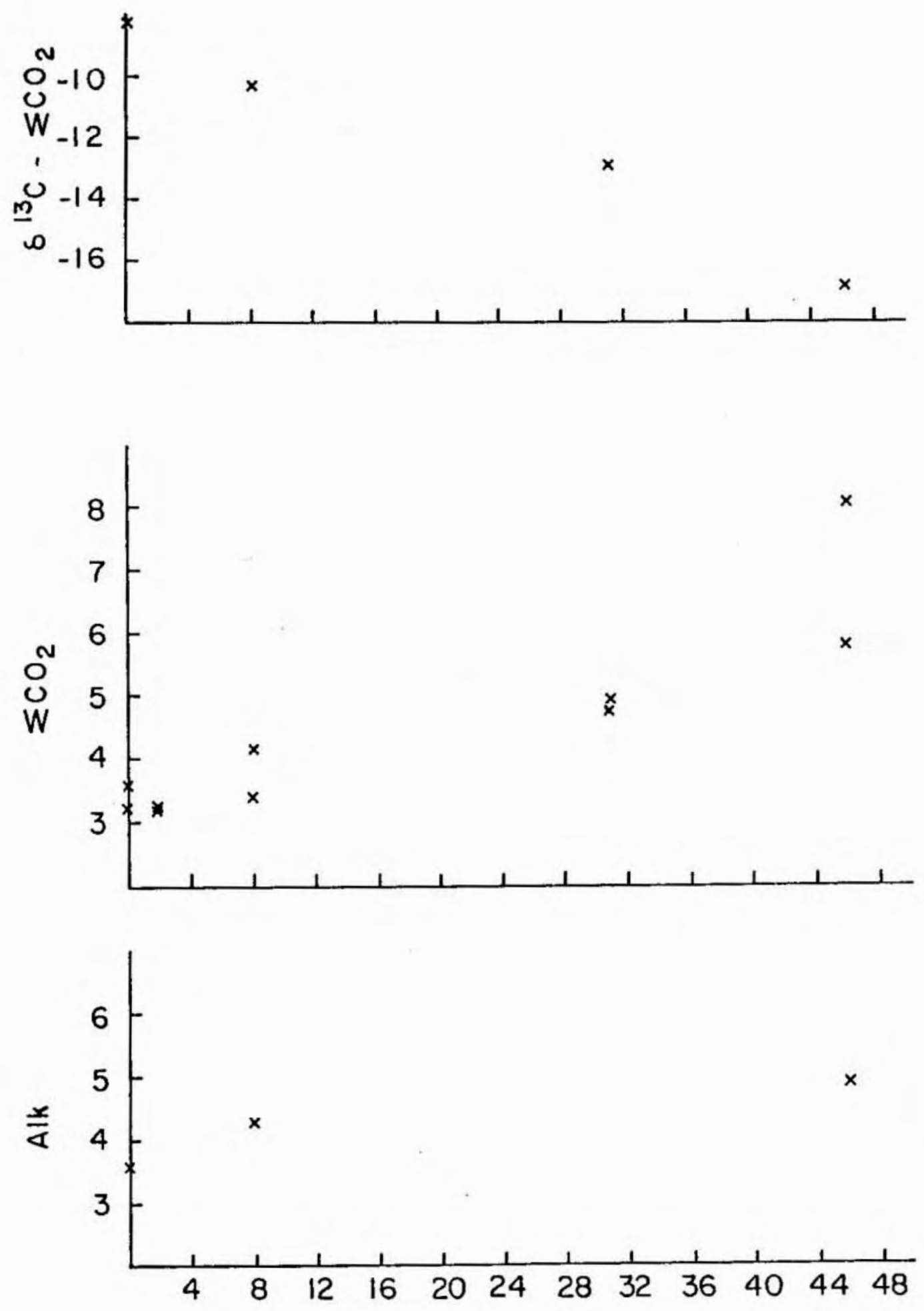
than in the deeper sediments. In IEI, $\sum \mathrm{CO}_{2}$ increased from 4.5 to 8.6 mmol/l over 2 days in the surface sediment and from approximately 3.0 to 5.0 mmol/1 over 35 days in the deeper sediment. The $\delta^{13} \mathrm{C}-\sum \mathrm{CO}_{2}$ decreased from -4.2 to -9.7 o/00 over 2 days in the surface sediment and from approximately -5.1 to -9.5 o/00 over 35 days in the deeper sediments. In IE2, $\left[\mathrm{CO}_{2}\right.$ increased from 3.5 to $30.8 \mathrm{mmol} / 1$ over 46 days in the surface sediments and from approximately 3 to $7 \mathrm{mmol} / 1$ over 46 days in the deeper sediments. The $\delta^{13} \mathrm{C}-\left[\mathrm{CO}_{2}\right.$ decreased from -2.0 to -19.0 o/oo over 46 days in the surface sediments and from approximately -8.1 to -16.0 o/oo over 46 days in the deeper sediments. In IEl, the initial rate of change of these constituents is greater than in IE2. To estimate error due to sample heterogeneity, eight to ten tubes of mud were collected at a fourth depth interval in both experiments and sacrificed at $t_{0}$. Data from these samples are listed in Table AII. The $\delta^{13} \mathrm{C}$ of $\sum \mathrm{CO}_{2}$ decreases over time in both IE1 and IE2 (Figure IV.3). This is due to the addition of ${ }^{13} \mathrm{C}$-depleted organic matter from the oxidation of organic carbon in the sediments. The $\delta^{13} \mathrm{C}-\mathrm{SOC}$ was measured on several samples and was found to be $-20.60 / 00$, identical to that found in the field studies.

1. Comparison of IEI and IE2

The $\mathrm{CO}_{2}$ production rates $\left(\mathrm{R}_{c}\right)$ calculated for each experiment are listed in Table IV.2. The rates were calculated by fitting the $\mathrm{CCO}_{2}$ vs. time data to a straight line; the slope of the line is $R_{c}$ in mol/L-day. The rates listed for $I E l$ depths $9-11 \mathrm{~cm}$ and $17-20 \mathrm{~cm}$ were calculated using data from time zero to two days. The initial rates in IEl were much higher than in IE2. In IEl, microbial activity appears to stop after 2 to 10 days, while in 
Table IV.2. Values of $R_{c}$ calculated from measured $\sum \mathrm{CO}_{2}$ concentrations over time in Incubation Experiments 1 and 2. Also listed are the values used to model $\mathrm{SCO}_{2}$ data in Chapter III. The values are reported in mol/L-sec and in parentheses in $\mathrm{nmol} / \mathrm{cm}^{3}$-day.

\begin{tabular}{|c|c|c|}
\hline Experiment & $\underline{R}_{c} \times 10^{9}$ & \\
\hline IEI, 0-2 & 24 & (1774) \\
\hline IE2, $0-3$ & $6.6-12$ & $(486-872)$ \\
\hline IEI, 9-11 & 3.3 & $(240)$ \\
\hline IE2, $12-15$ & $.41-.82$ & $(30-60)$ \\
\hline IEI, $17-20$ & 5.5 & $(404)$ \\
\hline IE2, $18-21$ & $.52-.76$ & $(38-56)$ \\
\hline October 83 & 4.0 & (294) \\
\hline June 84 & 4.25 & (312) \\
\hline August 84 & 7.5 & $(551)$ \\
\hline
\end{tabular}


the second experiment there is a constant rate of production over the 46 days of the experiment. Table IV.2 also lists the values of $R_{c}$ reported in Chapter III from modelling the observed profiles of $\sum \mathrm{CO}_{2}$ from the field studies. The rates in IE2 are more consistent with these independent rate estimates. The higher rates observed in IEI are even more surprising considering that the core was collected in February, a cold month, when microbial activity is reduced. The incubation samples from IEl were maintained at a temperature of $15^{\circ} \mathrm{C}$ for the course of the experiment to reduce the possibility of a temperature artifact. These facts are evidence that initial homogenization of the sediments in IEl artificially enhanced microbial activity.

Organic carbon oxidation is assumed to occur by sulfate reduction in these experiments. Dissolved sulfide concentrations suggest that sulfate reduction is important in both experiments. In both experiments dissolved sulfide is present in the deeper sediment intervals, and, in most cases, its concentration increases over time (Appendix I). Its presence in surface sediments is not detected until day 8 in IE2 and is very low in IEl. It is possible that there is a sink for sulfide, such as reduced iron, available in the surface sediment that is no longer available in the deeper sediments.

It is also possible to use the ratio of $\Delta A I k / \Delta \sum \mathrm{CO}_{2}$ as evidence for the occurrence of sulfate reduction. The stoichiometric equations listed in Table I.l can be used to calculate the relative changes expected in Alk and $\mathrm{CCO}_{2}$. It is assumed that organic matter with Redfield stoichiometry $(C: N: P=106: 16: 1)$ is degraded and that there is no dissolution or precipitation of $\mathrm{CaCO}_{3}$. Changing the $\mathrm{C}: \mathrm{N}$ ratio of the organic matter by a 
factor of two does not significantly alter the predicted $\triangle \mathrm{Alk} / \Delta\left[\mathrm{CO}_{2}\right.$. However, $\mathrm{CaCO}_{3}$ precipitation or dissolution could have a large impact on the calculated ratio. The $\mathrm{CaCO}_{3}$ saturation index, $\Omega$, is listed in Table IV. 3 for each sample. This value has been calculated assuming that the dissolved Ca concentration remains constant at $9.0 \mathrm{mM}$. In most samples after $t_{0}, a$ gross supersaturation is calculated. If it is assumed that the system is in equilibrium with calcite $\left(K_{s p}=4.07 \times 10^{-7}\right.$, Ingle, 1975), the expected concentration of $\mathrm{Ca}$ can be calculated. The dissolved $\mathrm{Ca}$ concentration was measured in a few samples and is listed in Table IV.4 along with the equilibrium concentration. The dissolved $\mathrm{Ca}$ does not change in these samples despite a gross disequilibrium in the $\mathrm{CaCO}_{3}$ system.

With these assumptions, $\triangle A l k / \Delta \sum \mathrm{CO}_{2}$ has been calculated for five oxidants important in marine sediments and the calculated ratios are listed in Table IV.5. Also listed in this table are the ratios observed in the incubation experiments. The results for IEl suggest that a combination of processes, such as oxygen and sulfate reduction, is occurring. It is possible that sample handling introduced oxygen to the samples before starting the incubation. The sediments were transferred to a beaker prior to introduction to the glove bag (see Section II.2.B. for sample handling). Using the calculated values of $R_{c}$ and assuming that the pore waters became saturated with $\mathrm{O}_{2}$, approximately $50 \%$ of the $\mathrm{CO}_{2}$ production in the deeper sediments could be the result of oxygen reduction. Because of the high rate of $\mathrm{CO}_{2}$ production in the surface sediments, $\mathrm{O}_{2}$ would be an insignificant oxidant.

The results for IE2 are consistent with nitrate and sulfate reduction. However, based on measured concentrations of nitrate in the pore water 
Table IV.3. Values of $\Omega\left(\mathrm{CaCO}_{3}\right.$ saturation index $)$ calculated for the incubation experiments. $\Omega$ is the ratio of the ion concentration product to the equilibrium concentration product $\left(K_{s p}\right)$.

\begin{tabular}{|c|c|c|c|c|c|}
\hline Sample & & $\underline{\Omega}$ & Sample & & $\Omega$ \\
\hline IE1 & day & & IE2 & day & \\
\hline $0-2 \mathrm{~cm}$, & 0 & 1.1 & $0-3 \mathrm{~cm}$ & 0 & 0.25 \\
\hline & 0 & 2.7 & & 2 & 1.6 \\
\hline & 1 & 0.34 & & 8 & 3.2 \\
\hline & 1 & 0.27 & & 19 & 2.8 \\
\hline & & & & 19 & 6.7 \\
\hline $9-11$ & 0 & 5.4 & $12-15$ & 2 & 1.7 \\
\hline & 0 & 4.2 & & 8 & 6.2 \\
\hline & 1 & 2.5 & & 31 & 2.8 \\
\hline & 1 & 1.7 & & 31 & 1.6 \\
\hline & 2 & 0.36 & & & \\
\hline & 2 & 0.61 & & & \\
\hline $17-20$ & 0 & 5.5 & $18-21$ & 0 & 1.1 \\
\hline & 0 & 5.2 & & 31 & 3.4 \\
\hline & 1 & 4.7 & & & \\
\hline & 1 & 0.4 & & & \\
\hline & 2 & 2.0 & & & \\
\hline
\end{tabular}


Table IV.4. Measured and calculated concentrations of dissolved $\mathrm{Ca}$ in mmol/1. Information in the sample column lists the experiment, depth interval of interest, and day in the experiment when the sample was collected.

Sample

IE1 $0-2,1$ 2 $17-20,1$

IE2 $0-3,0$

19

$12-15,8$

31
Ca, measured

10.8

10.7

9.75

10.3

10.1

10.0

10.0

\section{Ca, calculated}

undersatd.

n.d.

undersatd

undersatd.

3.25

1.45

5.67 
Table IV.5. Expected changes in $\Delta \mathrm{A} 1 \mathrm{k} / \Delta\left[\mathrm{CO}_{2}\right.$ for five different oxidants and observed changes for IEI and IE2.

Oxidant

$\mathrm{O}_{2}$

$\mathrm{NO}_{3}$

$\mathrm{Mn}$

$\mathrm{Fe}$

$\mathrm{SO}_{4}$

IEI

IE2

IE2

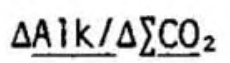

$-0.13$

0.89

4.4

8.0

1.15

$0.46 \pm 0.2 \quad($ range $0.16-0.75, n=7)$

$1.17 \pm 0.3 \quad$ (range $0.91-1.97, n=9$ )

$1.07 \pm 0.1 \quad($ range $0.91-1.24, n=8$ ) 
(Henrichs, 1980), there is not enough nitrate in the sediments to account for the amount of $\mathrm{CO}_{2}$ production observed. The absence of dissolved sulfide in the surface sediment samples until day 8 suggests that if sulfate reduction is occurring a sink fro dissolved sulfide is also being produced. The most likely sink is reduced iron. If it is assumed that iron reduction provides a quantitative sink of $\mathrm{Fe}^{2+}$ for dissolved sulfide the expected $\Delta A l k / \Delta \sum \mathrm{CO}_{2}$ is 1.0. This is consistent with the observed ratio. To supply the $\mathrm{Fe}_{2} \mathrm{O}_{3}$ used by the reaction, the sediments must be $0.75 \% \mathrm{Fe}$ by weight; the observed concentration is approximately $2.8 \%$ (Martin, 1985). This calculation assumes that the reaction occurs for the entire 46 days of the experiment. It is also possible that oxygen was introduced into the surface sediments in IE2 because of the different sampling method that was used. However, because of the high $\mathrm{CO}_{2}$ production rate, it could only be an important oxidant for the first 2 days (up to $20 \%$ of the $\mathrm{CO}_{2}$ production). Thus, it is likely that sulfate is a major, but not the only, oxidant in IE2, and the major oxidant in IEI cannot be determined.

IV.3. Modelling the Field Data

Generally, it is assumed that the $\delta^{13} \mathrm{C}$ of $\left[\mathrm{CO}_{2}\right.$ in pore waters can be related directly to the oxidation of organic carbon in the sediments (McCorkle, 1985; Section 1.1). In the simplest case, the oxidation of organic carbon occurs without fractionation, and the $\delta^{13} \mathrm{C}$ of the $\mathrm{CO}_{2}$ arising from the oxidation of organic carbon is exactly the same as the $\delta^{13} \mathrm{C}$ of SOC. In this case, the model used in Chapter III to describe the $\sum \mathrm{CO}_{2}$ profile can be extended to predict the $\delta^{13} \mathrm{C}-\sum \mathrm{CO}_{2}$ profile. 


\section{IV.3.A. Diagenetic Model}

The model used to describe the depth profile of $\mathrm{CCO}_{2}$ was described in detail in Section III.2B.1. In the model, it is assumed that the major processes affecting the concentration of $\mathrm{CCO}_{2}$ in the interstitial waters are diffusion, irrigation, production of $\mathrm{SCO}_{2}$ from the oxidation of organic carbon, and dissolution of $\mathrm{CaCO}_{3}$. To use this model to calculate the profile of $\delta^{13} \mathrm{C}$, the following equations must be solved for the individual isotopes, ${ }^{13} \mathrm{C}$ and ${ }^{12} \mathrm{C}$, e.g.:

I

II

$$
\begin{array}{ll}
\partial^{\prime} C / \partial t=D_{c}\left(\partial^{2}{ }^{\prime} C / \partial z^{2}\right)-\alpha^{*}\left({ }^{\prime} C-{ }^{\prime} C_{0}\right)+{ }^{\prime} R_{c} & 4-1 a \\
\partial^{\prime} C / \partial t=D_{c}\left(\partial^{2} C / \partial z^{2}\right)-\alpha^{*}\left({ }^{\prime} C-{ }^{\prime} C_{0}\right)+{ }^{\prime} R_{c} e^{-\beta z} & 4-1 b
\end{array}
$$

where the superscript $i$ refers to the carbon atom under consideration, i.e. ${ }^{12} \mathrm{C}$ or ${ }^{13} \mathrm{C}$

The values for ' $C_{0}$ and ' $R_{c}$ can be calculated from measured values for $\delta^{13} \mathrm{C}-\mathrm{BW}$ and $\delta^{13} \mathrm{C}-\mathrm{SOC}$ and the definition of $\delta^{13} \mathrm{C}$ (see $\mathrm{p} .2$ ).

$$
\begin{aligned}
& { }^{\prime} C_{0}={ }^{t} f_{0} C_{0} \\
& { }^{\prime} R_{c}={ }^{\prime} f_{0 r g} R_{c}
\end{aligned}
$$

where

$$
\begin{aligned}
& f_{n}=R_{n} /\left(1+R_{n}\right) \\
& R_{n}=\left({ }^{3} \mathrm{C} /{ }^{12} \mathrm{C}\right)_{n}=R_{s t d}\left(1+10^{-3} \delta^{13} C_{n}\right) \\
& R_{s t d}=.0112372 \quad \text { (Craig, 1957) }
\end{aligned}
$$

It is also assumed that the diffusion coefficients of $\mathrm{H}^{13} \mathrm{CO}_{3}^{-}$and $\mathrm{H}^{12} \mathrm{CO}_{3}^{-}$are the same. The actual difference is inversely proportional to the ratio of the square root of the masses; however, this is small and can be ignored. Solutions to these equations are similar to those in Chapter III, 
and are included in Appendix AII. The equations were solved using a steady-state assumption. As discussed in Section III.2.B.1, this assumption is only valid for the data from the months of October 83 , June 84 , and August 84. It is important to re-emphasize the fact that the model is defined only to $20 \mathrm{~cm}$ and is most likely overestimating irrigation at this depth. Overestimating irrigation at $20 \mathrm{~cm}$ will force the model to more positive values of $\delta^{13} \mathrm{C}$ at depth in the sediment than are observed.

In order to apply this model to the data, values of $\delta^{13} \mathrm{C}-\mathrm{SOC}$, $\delta^{13} \mathrm{C}-\mathrm{BW}$, and $\delta^{13} \mathrm{C}-\mathrm{CaCO}_{3}$ must be defined. The $\delta^{13} \mathrm{C}-\mathrm{SOC}$ in these sediments averages $-20.6 \pm 0.20 / 00$ (this study). However, $\delta^{13} \mathrm{C}$ of the organic carbon oxidized in the sediments may vary from the measured value. The model has been solved so that different values of $\delta^{13} \mathrm{C}-\mathrm{SOC}$ can be used in the surface and deep sediment. The values of $8^{13} \mathrm{C}-\mathrm{SOC}$ used in the model calculations are listed on the calculated profiles. The average $\delta^{13} \mathrm{C}-\mathrm{BW}$ measure in June 84 and August 84 is $1.3 \%$ used for October 83 , also. The $\delta^{13} \mathrm{C}-\mathrm{CaCO}_{3}$ averages $+1.7 \mathrm{o} / 00$; this value represents an average of thirteen measurements determined on individual shell fragments picked from the sediments. The values of all the other parameters used are the same as those listed in Table III.2. The calculated profiles of $\delta^{13} \mathrm{C}-\left[\mathrm{CO}_{2}\right.$ for October 83 , June 84 , and August 84 , the months for which a steady-state approximation is valid, are shown in Figure IV. 4 a to c. For every month, a value of $\delta^{13} \mathrm{C}-\mathrm{SOC}$ much greater than -20.6 o/oo must be used to fit the surface sediment data $\left(8^{13} \mathrm{C}-50 \mathrm{Cl}\right.$ in figure IV). The values of $\delta^{13} \mathrm{C}-\mathrm{SOC}$ used to $\mathrm{fit}$ the data range from -15.0 to -8.0 $\circ / 00$, and, in June 84 , even using $-8 \% / 00$, the observed data are not 
Figure IV.4. The profiles of $\delta^{13} \mathrm{C}-\sum \mathrm{CO}_{2}$ calculated 
Figure IV.4.a

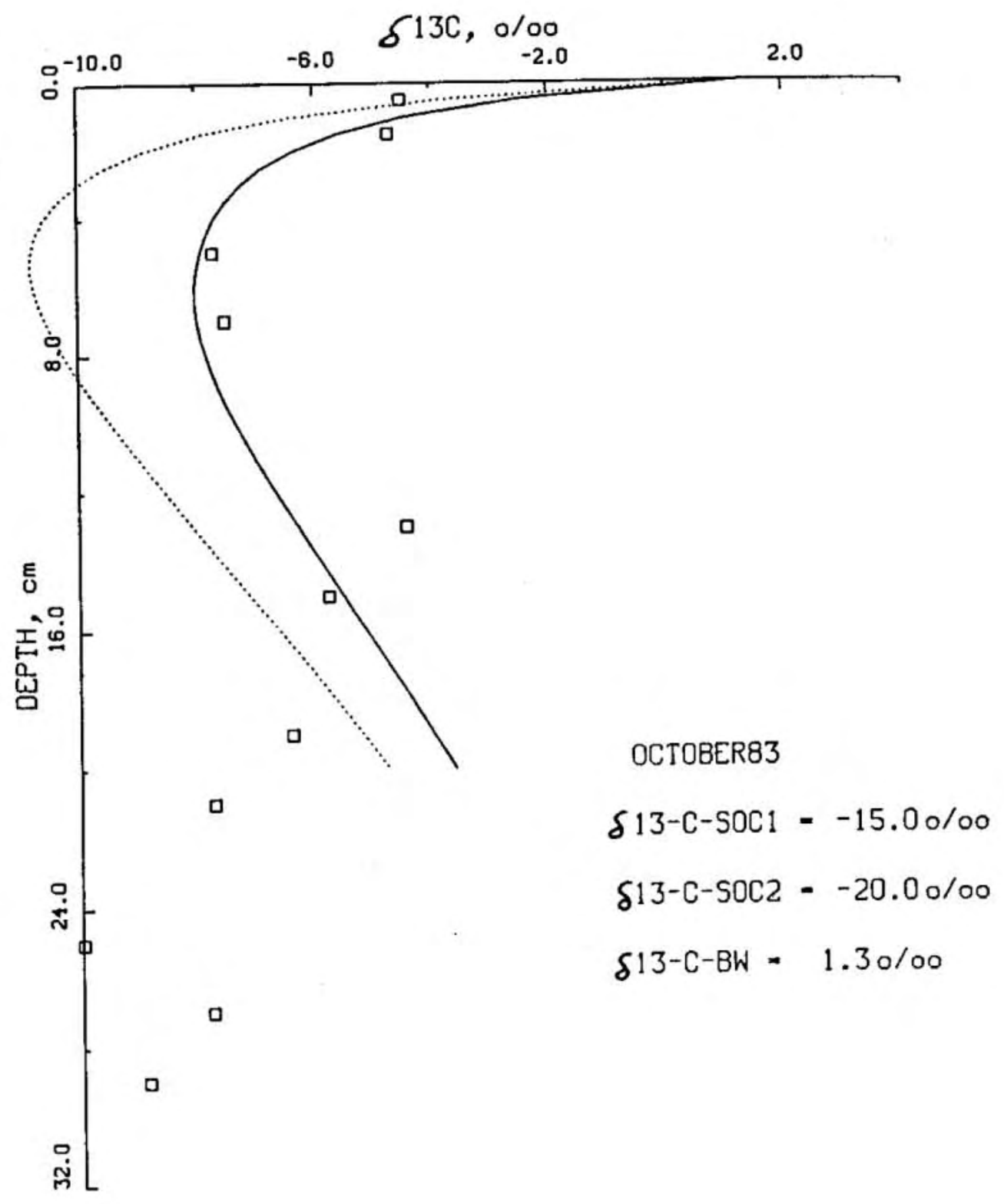


Figure IV.4.b

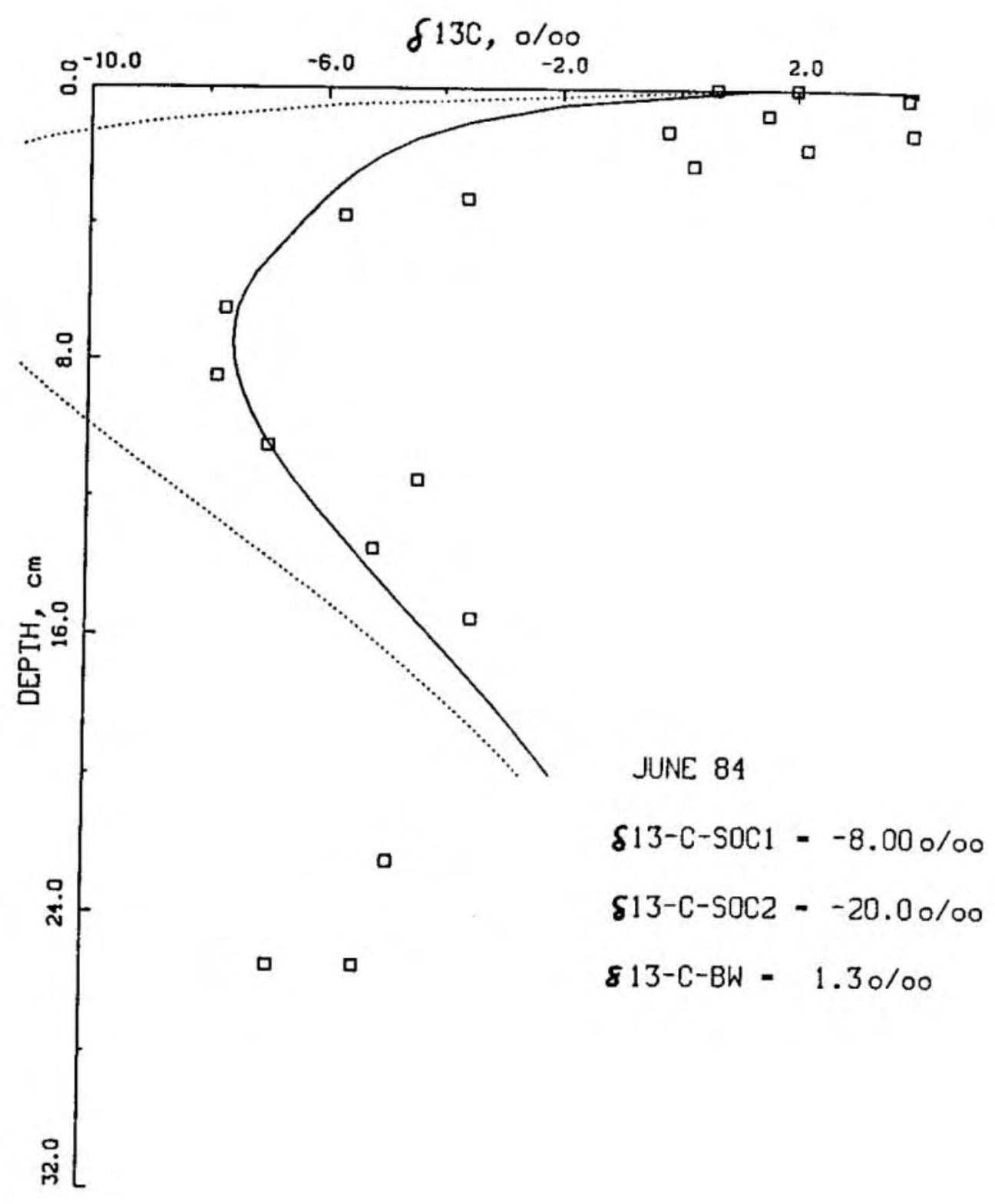


Figure IV.4.C

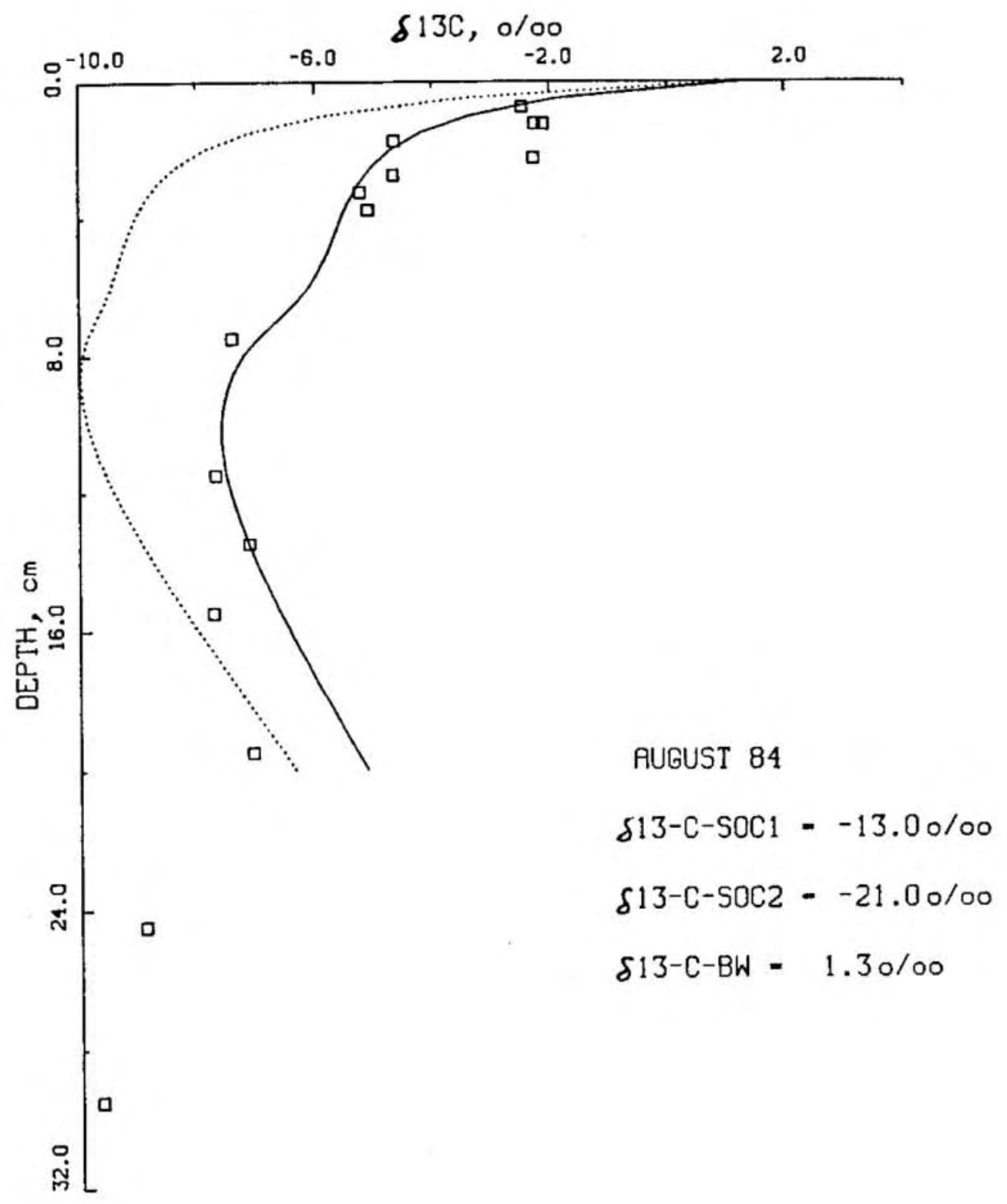


well-fit. In the October 83 and June 84 cores, the dissolved Ca profile suggests dissolution of $\mathrm{CaCO}_{3}$, a source of carbon enriched in ${ }^{13} \mathrm{C}$, in the sediments. However, the isotopic correction for dissolution only changes the $\delta^{13} \mathrm{C}$ of the added $\left[\mathrm{CO}_{2}\right.$ by less than 1.5 o/oo for both months. This is not large enough to explain the discrepancy between the predicted and observed values of $\delta^{13} \mathrm{C}-\mathrm{SOCl}$. In these three months, the measured $\delta^{13} \mathrm{C}-\sum \mathrm{CO}_{2}$ is greater than that expected due to the unfractionated oxidation of organic carbon in the surface sediments.

The use of different values of $\delta^{13} \mathrm{C}-\mathrm{SOC}$ in the surface and deep sediments suggests that there are different process affecting the $\delta^{13} \mathrm{C}$ of $\sum \mathrm{CO}_{2}$ in surface and deep sediments. The data below $20 \mathrm{~cm}$ indicate that there is a source of ${ }^{13} \mathrm{C}$-depleted $\sum_{\mathrm{CO}_{2}}$ at depth in the sediment. As discussed in Chapter III, this is most likely due to the oxidation of $\mathrm{CH}_{4}$ by sulfate just above the zone of methanogenesis. The oxidation of methane adds carbon that is highly depleted in ${ }^{13} \mathrm{C}$ to the $\mathrm{SCO}_{2}$ pool.

In December 83 and March 85 , a steady-state approximation is not valid. The value of $\delta^{13} \mathrm{C}_{0 \mathrm{~g} g}$, the apparent value of $\delta^{13} \mathrm{C}$ of $\mathrm{CO}_{2}$ added due to the oxidation of organic carbon, was calculated for these months by assuming that the flux of $\left[\mathrm{CO}_{2}\right.$ from pore water to bottom water $\left(\mathrm{J}_{\mathrm{t}}\right)$ was due to the addition of inorganic carbon in the sediments from the oxidation of organic carbon and the dissolution of $\mathrm{CaCO}_{3}$, i.e.

$$
\begin{aligned}
& J_{t}=J_{\text {Org }}+J_{C A C O 3} \\
& \delta^{13} \mathrm{CJ}_{\mathrm{t}}=\delta^{13} \mathrm{CJ}_{\text {Org }}+\delta^{13} \mathrm{CJ}_{C A C O 3}
\end{aligned}
$$

The dissolved $\mathrm{Ca}$ profile was used to estimate the amount of $\left\{\mathrm{CO}_{2}\right.$ added due to the oxidation of organic carbon. Using the above equations, it was 
calculated that $\delta^{13} \mathrm{C}_{0 \mathrm{rg}}$ is -12.1 in December 83 and -16.0 in March 85 .

Again, these values are much greater that the observed value of $\delta^{13} \mathrm{C}-\mathrm{SOC}$.

The depth profiles of $\mathrm{SCO}_{2}$ are described by a model that includes the processes of oxidation of organic carbon, irrigation, and diffusion. However, application of the same model to the $\delta^{13} \mathrm{C}$ of $\left[\mathrm{CO}_{2}\right.$ in Buzzards Bay sediments indicates that the $\mathrm{ECO}_{2}$ added to the pore waters is enriched in ${ }^{13} \mathrm{C}$ over the organic carbon in the sediments. It is possible that there are additional processes affecting the $\left[\mathrm{CO}_{2}\right.$ profile that cannot be distinguished through consideration of the $\left[\mathrm{CO}_{2}\right.$ profile alone. The most likely explanations of the $\delta^{13} \mathrm{C}-\sum \mathrm{CO}_{2}$ profile are:

1) the organic carbon oxidized is not isotopically the same as the bulk SOC, or,

2) there is an additional source of ${ }^{13} \mathrm{C}$-enriched carbon to the pore waters, i.e. from bottom water or exchange with $\mathrm{CaCO}_{3}$.

These possibilities are discussed below.

IV.4. Fractionated Oxidation of SOC

IV.4.A. Incubation Studies

It is possible that the $\delta^{13} \mathrm{C}$ of the organic carbon that is oxidized in these sediments is actually different from the bulk organic carbon. In order to define the $\delta^{13} \mathrm{C}$ of $\mathrm{CO}_{2}$ arising from the oxidation of $\mathrm{SOC}$ in Buzzards Bay sediments, incubation studies of sediment from the site of the field study were conducted. The experiments were carried out in order to observe the oxidation of organic carbon in a controlled environment, eliminating the complications of diffusion, irrigation, and mixing. The $\delta^{13} \mathrm{C}$ of the $\sum \mathrm{CO}_{2}$ added $\left(\delta^{13} \mathrm{C}_{\mathrm{add}}\right)$ over time during the incubations can be 
calculated using a mass balance. Use of a mass balance is valid because these experiments were carried out in a closed system. Since there is no dissolution or precipitation of $\mathrm{CaCO}_{3}$ over the course of the experiments (Section IV.2.B.1), it is assumed that the value of $\delta^{13} C_{a d a}$ represents the average $\delta^{13} \mathrm{C}$ of the total organic carbon that is oxidized in the sediments, i.e. $\delta^{13} C_{o r g}$. The mass balance equations are:

$$
\begin{aligned}
& \sum \mathrm{CO}_{2 m}=\sum \mathrm{CO}_{2 t a}+\sum \mathrm{CO}_{2 \mathrm{add}} \\
& \delta^{13} \mathrm{C}_{m} \sum \mathrm{CO}_{2 \mathrm{~m}}=\delta^{13} \mathrm{C}_{\mathrm{t}} \sum \mathrm{CO}_{2 \mathrm{ta}}+\delta^{13} \mathrm{C}_{\mathrm{add} d} \sum \mathrm{CO}_{2 \mathrm{add}}
\end{aligned}
$$

In Tables IV. 6 and 7 the calculated values of $\delta^{13} \mathrm{C}_{\text {add }}$ are listed with the measured values of $\sum \mathrm{CO}_{2}$ and $\delta^{13} \mathrm{C}-\sum \mathrm{CO}_{2}$ used to make the calculation. Each value of $\delta^{13} C_{a d d}$ was calculated using $t_{0}$ as a reference point. The results from both IEI and IE2 suggest that the $\delta^{13} \mathrm{C}$ of the $\mathrm{CO}_{2}$ added from the oxidation of organic carbon is more enriched in ${ }^{13} \mathrm{C}$ than the organic carbon in the sediments. However, the results from IEI and IE2 are not in agreement with each other. In IEI, it is calculated that the $\delta^{13} \mathrm{C}$ of the $\mathrm{CO}_{2}$ added is more positive than the $\delta^{13} \mathrm{C}$ of $\mathrm{SOC}$ at every depth of the sediment and at every time point, while in IE2, this observation is valid only for results from the surface sediment. It is likely that these differences are related to the different procedures used to sample the sediments. However, it is also possible that the observed differences are related to the fact that the sediments for IEI were collected in February and for IE2 in August.

The average of results from IEl indicate that the $\delta^{13} \mathrm{C}$ of the $\mathrm{CO}_{2}$ added from the oxidation of organic carbon is $-15.9 \pm 1.7$ o/oo. The results from IE2 indicate that $\delta^{13} \mathrm{C}_{\text {ado }}$ is $-20.2 \pm 1.7 \mathrm{o} / 00$. However, in IE2, 
Table IV.6. Calculated values of $\delta^{13} C_{a d d}$ for Incubation Experiment 1 as well as the measured values used to make the calculation. The errors were propogated through the calculation using estimated errors of $\pm 6 \%$ for $\sum \mathrm{CO}_{2}$ and $\pm 8 \%$ for $\delta^{13} \mathrm{C}-\sum \mathrm{CO}_{2}$.

$$
\underline{T i m e} \text { (days) } \quad\left[\underline{\mathrm{CO}}_{2}(\mathrm{mM}) \quad \delta^{13} \underline{\mathrm{C}}-\left[\underline{\mathrm{CO}}_{2} \quad \delta^{13} \mathrm{C}_{\mathrm{add}}\right.\right.
$$

$0-3 \mathrm{~cm}$

$\begin{array}{llll}0 & 4.59 & - & \\ 0 & 4.46 & -4.2 & \\ 1 & 7.68 & -8.2 & -13.9 \pm 3.1 \\ 2 & 8.55 & -9.9 & -15.8 \pm 3.1 \\ 2 & 8.66 & -9.5 & \end{array}$

$9-11 \mathrm{~cm}$

0

3.01

$-5.1$

35

5.12

$-9.7$

$-16.3 \pm 3.0$

$17-20 \mathrm{~cm}$

0

3.02

$-5.2$

2

4.60

2

3.6

$-7.3$

$-13.2 \pm 4.8$

11

4. 39

$-8.8$

$-16.7 \pm 4.9$

35

4.44

$-9.98$

35

4.34

$-9.2$

$-19.3 \pm 5.6$ 
Table IV.7. Calculated values of $\delta^{13} \mathrm{C}_{\mathrm{add}}$ for Incubation Experiment 2 as well as the measured values used to make the calculation. The errors were propogated through the calculation using estimated errors of $\pm 6 \%$ for $\sum \mathrm{CO}_{2}$ and $\pm 8 \%$ for $\delta^{13} \mathrm{C}-\sum \mathrm{CO}_{2}$.

$$
\begin{aligned}
& \text { Time (days) } \quad\left[\mathrm{CO}_{2}\right. \\
& \delta^{13} \underline{C}-\left[\underline{C O}_{2} \quad \delta^{13} \mathrm{Codd}\right.
\end{aligned}
$$

$0-3 \mathrm{~cm}$

$\begin{array}{llll}0 & 3.70 & -1.58 & \\ 0 & 3.28 & -2.32 & \\ 2 & 5.52 & -7.31 & -19.8 \pm 4.8 \\ 2 & 4.90 & -8.05 & \\ 8 & 10.14 & -10.92 & -16.6 \pm 2.5 \\ 8 & 9.00 & -11.66 & \\ 19 & 22.62 & -14.06 & -16.9 \pm 1.7 \\ 19 & 20.06 & -14.80 & \\ 42 & 32.63 & -18.68 & -21.2 \pm 2.1 \\ 42 & 28.93 & -19.42 & \end{array}$

$12-15 \mathrm{~cm}$

$$
0
$$

3.16

2.80

$-8.47$

$-7.73$

cont. on next page 
Table IV.7. cont.

\begin{tabular}{|c|c|c|c|c|}
\hline \multirow[b]{2}{*}{$18-21 \mathrm{~cm}$} & Time & $\sum \underline{\mathrm{CO}}_{2}$ & $\delta^{13} \underline{\mathrm{C}}-\left[\underline{\mathrm{CO}} \underline{\mathrm{O}}_{2}\right.$ & $\delta^{13} C_{a d d}$ \\
\hline & $\begin{array}{l}0 \\
0\end{array}$ & $\begin{array}{l}3.78 \\
3.36\end{array}$ & $\begin{array}{l}-8.71 \\
-7.97\end{array}$ & \multirow{3}{*}{$-26.3 \pm 9.8$} \\
\hline & 31 & 5.05 & -13.20 & \\
\hline & 31 & 4.47 & -12.46 & \\
\hline & 46 & 8.56 & -17.23 & \multirow{2}{*}{$-23.6 \pm 4.1$} \\
\hline & 46 & 7.60 & -16.49 & \\
\hline
\end{tabular}


the results suggest that, in the surface sediments, the $\delta^{13} \mathrm{C}$ of the added $\mathrm{CO}_{2}\left(\delta^{13} \mathrm{Cadd}_{\mathrm{ad}}=-18.6 \pm 1.5 \mathrm{o} / 00\right)$ is heavier than the carbon in the sediments, while, in the deeper sediments, it appears that the $\delta^{13} \mathrm{C}$ of the added $\mathrm{CO}_{2}(-21.8 \pm 3.0 \mathrm{o} / 00)$ is equal to or more negative than the sediment carbon. This observation is consistent with the model results (Section IV.2.A.1) which suggested that the $\delta^{13} \mathrm{C}$ of the added carbon was more ${ }^{13} \mathrm{C}$-enriched in the surface sediments than in the deeper sediments. The values of $\delta^{13} \mathrm{C}_{a d a}$ calculated from data from the $0-3 \mathrm{~cm}$ interval of sediments from IE2 are believed to represent most accurately the fractionation of SOC that would be observed in the field in the zone of rapid remineralization. These values are considered the best because the other data collected during this experiment indicate that IE2 reproduced field conditions more accurately than IEl. Additionally, in the surface interval, the rate of microbial activity is great enough for large changes to be observed in the measured species over a short period of time. It is possible that the 46 -day samples are affected by a time-dependent artifact. The rate of $\mathrm{CO}_{2}$ production is so high in the surface sediments that, if sulfate is the major oxidant, over one-half the existing pool is depleted. It is possible that the system is no longer really reflecting what occurs in the field. Then, using the data collected between $\mathrm{O}$ and 20 days, the $\delta^{13} \mathrm{C}$ of the $\mathrm{CO}_{2}$ added is $-17.8 \pm 1.90 / 00$. This is the value that will be used in further calculations. It is recognized that this value needs further documentation and may be as high as -15 o/00 or as low as $-190 / 00$.

The value of $-17.80 / 00$ is significantiy greater $(i \sigma)$ than the $\delta^{13} \mathrm{C}$ of the SOC in the sediments. The observed fractionation may be the result of 
selective remineralization at a molecular level. The microorganisms responsible for the oxidation of SOC may cleave carboxyl groups selectively from molecules. Carboxyl carbons are known to be enriched in ${ }^{13} \mathrm{C}$ with respect to the rest of the molecule (Abelson and Hoering, 1961). It is also possible that carbohydrates, another relatively heavy source of carbon (Degens, 1969), are degraded preferentially. However, the amount of carbon that is oxidized in the sediments makes it likely that the observed fractionation is a result of the multiple sources of carbon to the sediment water interface in coastal areas. It is probable that phytoplankton carbon is the first fraction of carbon to be degraded, and the remaining $S O C$ is a mixture of phytoplankton, terrestrial, and salt marsh carbon. The calculated value of $\delta^{13} \mathrm{C}_{0 \mathrm{rg}},-17.8 \mathrm{o} / 00$, is the same as that measured on a plankton tow sample from Buzzards Bay (this study, $\delta^{13} \mathrm{C}_{p}$ lankton $=-17.7 \mathrm{o} / 00$ ).

The perfect agreement is most likely fortuitous since only one tow was made at one time, yet, the value of $\delta^{13} \mathrm{C}_{0 \mathrm{~g}}$ is within the range of values measured for plankton (Libes, 1983; Williams and Linick, 1975).

In this section, it has been shown that the $\delta^{13} \mathrm{C}$ of the $\mathrm{CO}_{2}$ added due to the oxidation of organic carbon is most likely $-17.8 \% / 00$. This value is significantly enriched in ${ }^{13} \mathrm{C}$ over SOC in the sediments $\left(\delta^{13} \mathrm{C}=-20.6\right.$ $0 /(0)$. However, this fractionation is not enough to explain the value of $\delta^{13} C_{a d d}$ calculated from the field study (greater than $-150 / 00$ ). Inorganic processes that can affect the value of $\delta^{13} \mathrm{C}_{\text {add }}$ are discussed in the next sections.

IV.5. Diffusion of Bottom Water Carbonate Ion The enrichment of ${ }^{13} \mathrm{C}$ in $\sum \mathrm{CO}_{2}$ from Buzzards Bay pore waters cannot 
be explained completely by the fractionated oxidation of organic carbon. An additional source of ${ }^{13} \mathrm{C}$-enriched carbon may be carbonate ion from bottom water. The diffusion of $\mathrm{CO}_{3}^{2-}$ ion from bottom water is predicted based on the measured values of Alk, $2 \mathrm{CO}_{2}, \mathrm{H}_{2} \mathrm{~S}$, and $\mathrm{PO}_{4}$. Using these measured values, the solution $\mathrm{pH}$ as well as the speciation of $\sum \mathrm{CO}_{2}$ can be calculated. The $\mathrm{pH}$ and $\mathrm{CO}_{3}^{2-}$ concentration for bottom water and the top sediment interval are listed for each month in Table IV.8. It is required that, while there is a net flux of $\mathrm{CCO}_{2}$ from pore water to bottom water, there is a flux of $\mathrm{CO}_{3}^{2-}$ from bottom water to pore water due to the large concentration gradient. Most of this carbonate ion becomes protonated in the pore waters at $\mathrm{pH}$ 6.2-7.7 and some is transferred back across the interface while some is retained in the pore waters. This process is shown in Figure IV.5. It is important to realize that this exchange could occur across burrow walls also. The magnitude of this proposed flux can be calculated in two ways. In both calculations it is assumed that the flux of a species to and from bottom water is composed of the diffusive flux across the sediment water interface $\left(J_{d, f}\right)$ and the flux due to irrigation (I), i.e.

$$
J_{t}=J_{d i f}+I
$$

where

$$
\begin{aligned}
& J_{d, f} \simeq-\phi D_{s} \Delta C /\left.\Delta z\right|_{z=0} \\
& I=\int_{0}^{20}-\phi \alpha\left(C_{z}-C_{0}\right) d z \simeq \sum_{0}^{20}-\phi \alpha\left(C_{z}-C_{0}\right) \Delta z
\end{aligned}
$$

During the months when irrigation is unimportant, $J_{t}=J_{d i f}$.

The calculated concentration of $\mathrm{CO}_{3}^{2-}$ and the isotopic ratio of $\sum \mathrm{CO}_{2}$ can be used to calculate the flux of $\mathrm{CO}_{3}^{2-}$ to pore waters. The use of the carbonate ion is straightforward while the use of $\delta^{13} \mathrm{C}-\left[\mathrm{CO}_{2}\right.$ 
Table IV.8. Calculated values of carbonate ion and pH for bottom water and the pore water in the top sediment interval. The carbonate ion concentrations are reported in units of $\mathrm{mol} / \mathrm{L}$.

Month

$\underline{\mathrm{CO}}_{3}^{2-} \times 10^{6}$

October $83 \quad$ BW

$$
0-1
$$

38

7.4

December 83

$$
\text { BW }
$$

170

8.4

$0-0.5$

28

7.5

June 84

BW

91

8.0

0.0 .5

2

6.2

August 84

BW

152

8.1

$0-0.5$

8

6.7

March 85

BW

$0-0.5$

58

7.7

October 85

$\begin{array}{lrl}\text { BW } & 144 & 8.2 \\ 0-0.5 & 83 & 7.9\end{array}$


Figure IV.5. Representation of the diffusion of carbonate species to and from bottom water. 
Figure IV.5

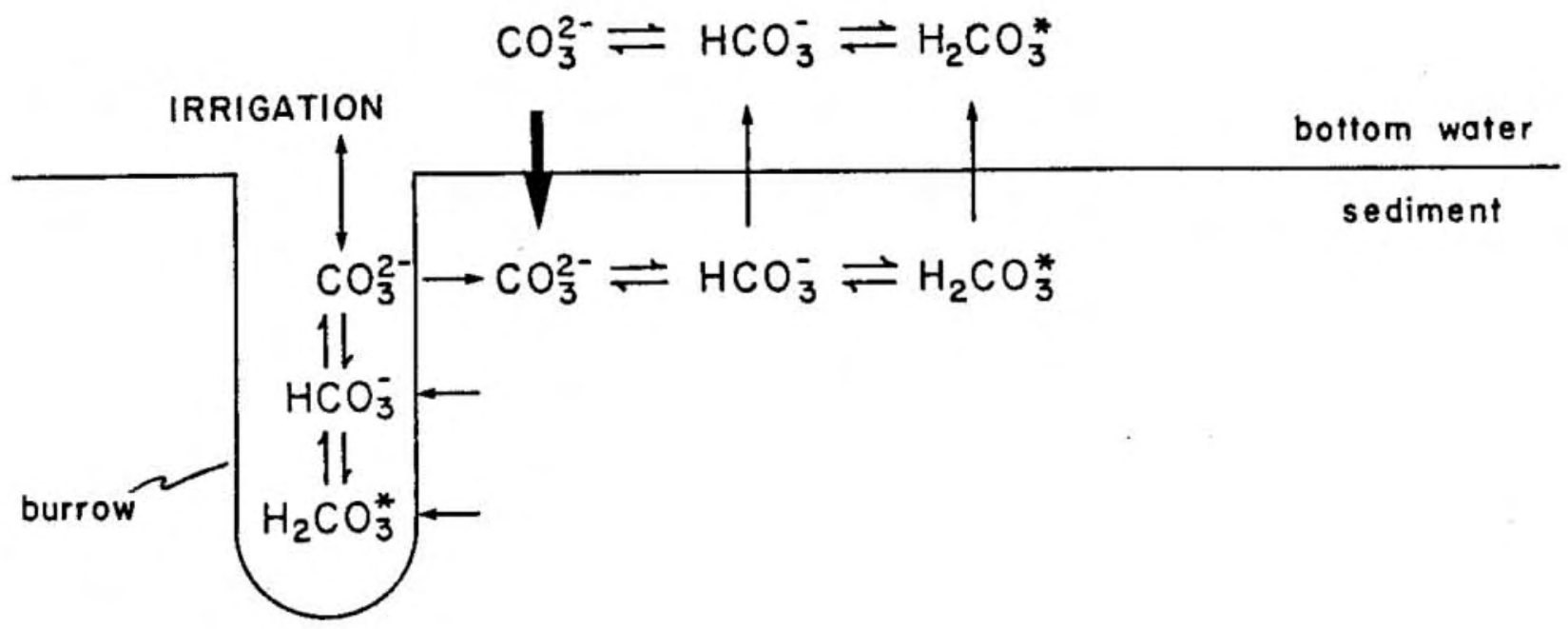


is more complicated. After calculating the flux for each isotope, the $\delta^{13} \mathrm{C}$ of $\mathrm{J}_{\mathrm{t}}$ can be calculated. Then a mass balance similar to equations 4- $a$ and $b$ can be used to calculate the amount of the total flux across the sediment water interface to bottom water, $J_{t}$, that can be attributed to recycled bottom water carbonate ion, $J_{b w}$. In this calculation it is assumed that $\delta^{13} \mathrm{C}_{-} \mathrm{CO}_{3}^{2-}$ is the same as that of $\left[\mathrm{CO}_{2}\right.$. The fractionation between $\mathrm{CO}_{3}^{2-}$ and $\mathrm{HCO}_{3}^{-}$has never been measured. However, based on theoretical calculations, the $\delta^{13} \mathrm{C}$ of $\mathrm{CO}_{3}^{2-}$ is approximately $1.5 \%$ less than the $\delta^{13} \mathrm{C}$ of $\mathrm{HCO}_{3}^{-}$(Deines et al., 1974). Because this fractionation has been ignored, the calculated values of $J_{b w}$ are minimum values. The calculations were made twice, first, assuming that the $\delta^{13} \mathrm{C}$ of $\mathrm{CO}_{2}$ produced from the oxidation of organic carbon was the value determined from the incubation experiments, $-17.8 \% / 00$, and then, assuming that the oxidation of organic carbon occurred without fractionation, i.e. $\delta^{13} \mathrm{C}$ of $\mathrm{CO}_{2}$ is $-20.60 / 00$. The mass balances necessary to make this calculation are:

$$
\begin{aligned}
& J_{t}=J_{c a}+J_{o r g}+J_{b w} \\
& \delta^{13} C_{J t} J_{t}=\delta^{13} C_{c a} J_{c a}+\delta^{13} C_{o r g} J_{a r g}+\delta^{13} C_{b w} J_{b w}
\end{aligned}
$$

The values calculated for $J_{b w}$ are listed in Table IV.9. This table also lists what percentage of the total flux across the sediment water interface this value represents, i.e. $\left(J_{b w} / J_{t}\right) 100$. The values reported for June 84 represent the irrigation flux (I) between 6 and $16 \mathrm{~cm}$. Data from the top four cm were not used because the isotopic values are much heavier than could be obtained in this sediment system by any of the processes described so far, i.e. $\delta^{13} C_{0-0.5}>\delta^{13} C_{c_{a c o 3}}, \delta^{13} C_{b w}$. These data are 
Table IV.9. Values calculated for $\mathrm{J}_{\mathrm{bw}}$, the amount of the total flux of $\mathrm{CO}_{2}$ across the sediment water interface that can be attributed to recycled bottom water carbonate ion. The fluxes were calculated using the concentrations of carbonate ion and again with the total dissolved concentrations of ${ }^{13} \mathrm{C}$ and ${ }^{12} \mathrm{C}$. The values in parentheses represent the values of $\delta^{13} \mathrm{C}$ of $\mathrm{CO}_{2}$ produced from the oxidation of organic carbon assumed for the calculation. The fluxes are reported in $\mathrm{mol} / \mathrm{cm}^{2}-\mathrm{sec}$.

Month

October 83

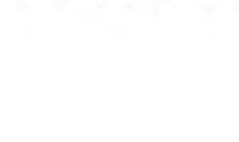

December 83

June 84

August 84

March 85

\begin{abstract}
Conc. used
\end{abstract}
$\mathrm{CO}_{3}^{2-}$

${ }^{13} \mathrm{C}(-20.6)$

${ }^{13} \mathrm{C}(-17.8)$

$\mathrm{CO}_{3}^{2-}$

${ }^{13} \mathrm{C}(-20.6)$

${ }^{13} \mathrm{C}(-17.8)$

$\mathrm{CO}_{3}^{2-}$

${ }^{13} \mathrm{C}(-20.6)$

${ }^{13} \mathrm{C}(-17.8)$

$\mathrm{CO}_{3}^{2-}$

${ }^{13} \mathrm{C}(-20.6)$

${ }^{13} \mathrm{C}(-17.8)$

$\mathrm{CO}_{3}^{2-}$

${ }^{13} \mathrm{C}(-20.6)$

${ }^{13} \mathrm{C}(-17.8)$
$\underline{J}_{\text {bw }} \times 10^{12}$

0.5

3.1

0.6

2.2

3.4

2.7

1.2

1.7

1.0

5.2

13.5

9.8

1.6

3.0

1.4
\% J $\underline{\mathrm{J}}_{\mathrm{s}} \quad \delta^{13} \underline{\mathrm{C}}_{\mathrm{J}}$

2

14

$-14.8$

3

22

34

$-10.1$

27

15

23

$-13.3$

14

11

28

$-10.5$

22

8

16

$-10.3$

7 
discussed later.

The fluxes calculated from $\mathrm{CO}_{3}^{2-}$ and $\delta^{13} \mathrm{C}-\left[\mathrm{CO}_{2}\right.$ generally agree, although, the best agreement is found when a value of $-17.8 \mathrm{o} / 00$ is used for $\delta^{13} \mathrm{C}$ org. The values calculated using $\delta^{13} \mathrm{C}$ are more precise because the concentrations of ${ }^{13} \mathrm{C}$ and ${ }^{12} \mathrm{C}$ are known to about $\pm 5 \%$ while the concentrations of $\mathrm{CO}_{3}^{2-}$ are known to only about $\pm 25 \%$.

It is probable that diffusion of bottom water carbonate ion to the pore waters is responsible in part for the isotopically heavy values calculated for $\delta^{13} \mathrm{C}_{\mathrm{org}}$ from the field data, $-14.7 \mathrm{o} / 00$. However, if this is the only process that is adding heavy carbon to the pore waters, then up to $30 \%$ of the $\sum \mathrm{CO}_{2}$ flux to the bottom water must be recycled bottom water carbonate ion.

IV.6. Isotopic Exchange Between $\mathrm{CCO}_{2}$ and $\mathrm{CaCO}_{3}$

The possibility for isotopic exchange as a second source of ${ }^{13} \mathrm{C}$-rich $\sum \mathrm{CO}_{2}$ aiso exists. Since the $\delta^{13} \mathrm{C}$ of $\mathrm{CaCO}_{3}$ is $1.7 \mathrm{o} / 00$ and the $\delta^{13} \mathrm{C}$ of $\sum \mathrm{CO}_{2}$ is usually less than 0.0 o/oo this process could enrich the ${ }^{13} \mathrm{C}$ concentration of $\mathrm{SCO}_{2}$. Using acidification techniques, the amount of $\mathrm{CaCO}_{3}$ in the sediments is difficult to ascertain because, except for a distinct shell layer at about $25 \mathrm{~cm}$, there is very little carbonate in the sediments. The amount of $\mathrm{CaCO}_{3}$ in shell-free sediment is estimated to be approximately $0.2 \%$ by weight, or about five times the $\sum \mathrm{CO}_{2}$ pool. In these sediments, the $\delta^{13} \mathrm{C}$ of $\mathrm{SCO}_{2}$ is about 8 o/ oo lighter than the $\delta^{13} \mathrm{C}$ of $\mathrm{CaCO}_{3}$ while the equilibrium fractionation dictates a value only $1.3 \mathrm{o} / 00$ lighter. Whether this system will achieve isotopic equibrium in the absence of a gas phase and how long this equilibrium would take are unknown. However, there is some evidence that suggests this exchange is not significant 
relative to the other processes affecting the $\delta^{13} \mathrm{C}$ of $\left[\mathrm{CO}_{2}\right.$.

The $\delta^{13} \mathrm{C}$ of $\sum \mathrm{CO}_{2}$ has been measured in cores collected as part of the Deep Sea Drilling Project (DSDP) (Claypool et al., 1983). The gradients in $\delta^{13} \mathrm{C}-\left[\mathrm{CO}_{2}\right.$ and $\sum \mathrm{CO}_{2}$ can be explained without considering exchange with $\mathrm{CaCO}_{3}$, a phase present in the core examined. The $\sum \mathrm{CO}_{2}$ in DSDP cores has certainly been in contact with $\mathrm{CaCO}_{3}$ for a much longer period than the sediment in Buzzards Bay. Further, Emrich et al. (1970) observed no isotope exchange between a dissolved $\mathrm{HCO}_{3}^{-}\left(\delta^{13} \mathrm{C}=4.4 \mathrm{o} / 00\right)$ solution and solid-phase $\mathrm{CaCO}_{3}\left(\delta^{13} \mathrm{C}=-9.10 / 00\right)$ in a closed system over twelve days. While net isotopic exchange between $\mathrm{CCO}_{2}$ and $\mathrm{CaCO}_{3}$ cannot be ruled out entirely as an explanation, existing evidence deems it unlikely. IV.7. June 84 Core

The $\delta^{13} \mathrm{C}$ of $\left[\mathrm{CO}_{2}\right.$ measured in pore water between 0 and $4 \mathrm{~cm}$ in a core collected in June 84 is much greater than that observed in any other month (see Figures III.3 and IV.1). Also, the dissolved iron concentration measured in the sediment interval $0.5-1 \mathrm{~cm}$ was the highest ever observed in these sediments (Martin, 1985). The ${ }^{222} \mathrm{Rn} /{ }^{26} \mathrm{Ra}$ data could only be explained by assuming that irrigation was constant to $20 \mathrm{~cm}$, although, in other months, the data were modelled with an exponentially decreasing term (Martin, 1985). The bottom water Alk is greater than that measured in the 0 to $0.5 \mathrm{~cm}$ sediment interval unlike other months where Alk is always greater in pore waters than in bottom water. As discussed in Chapter III and Martin (1985), it is likely that the oxidation of solid-phase iron sulfides is significant in this core. This process creates a strong acid and the effect of this acid on the carbonate equilibrium can be used to explain the isotopic 
results.

The $\mathrm{pH}$ calculated for the sediment interval $0-0.5 \mathrm{~cm}$ is 6.2 (Table IV.9). At this $\mathrm{pH}, \mathrm{H}_{2} \mathrm{CO}_{3}^{*}$ (where $\mathrm{H}_{2} \mathrm{CO}_{3}^{*}=\mathrm{H}_{2} \mathrm{CO}_{3}+\mathrm{CO}_{2 \text { diss }}$ ) and $\mathrm{HCO}_{3}^{-}$are the major inorganic carbon species (Figure IV.6). Thus, the flux of $\left[\mathrm{CO}_{2}\right.$ from the sediments to bottom water in June 84 contained a significant amount of $\mathrm{H}_{2} \mathrm{CO}_{3}^{*}$. Using the isotope equilibrium constants reported in Deines et al. (1974) the fractionation between $\mathrm{H}_{2} \mathrm{CO}_{3}^{*}$ and $\mathrm{HCO}_{3}^{-}$is approximately $80 / 00$ with $\mathrm{H}_{2} \mathrm{CO}_{3}^{*}$ enriched in ${ }^{12} \mathrm{C}$. It is possible, then, that diffusion of $\mathrm{H}_{2} \mathrm{CO}_{3}^{*}$ from the sediments preferentially removes ${ }^{12} \mathrm{C}$ from the sediments. The time required to achieve the observed results is difficult to estimate. However, if diffusion of $\mathrm{H}_{2} \mathrm{CO}_{3}^{*}$ is coupled with diffusion of carbonate ion from bottom water to pore water then the time required for isotope exchange is shortened. Thus, it appears that the oxidation of solid-phase iron sulfides in coastal sediments has a pronounced effect on pore water chemistry.

It is possible that the same type of isotope exchange also affects the data collected in August 84. The pH of pore waters samples between 0 and $4 \mathrm{~cm}$ is less than 7.0 this month, also. In Table IV.10, the value calculated for $J_{\text {bw }}$ using $\delta^{13} \mathrm{C}-\left[\mathrm{CO}_{2}\right.$ does not agree well with that calculated using the carbonate ion concentration. In all other months the agreement is much better. Enrichment of ${ }^{13} \mathrm{C}$ in pore water $\mathrm{SCO}_{2}$ by the loss of $\mathrm{H}_{2} \mathrm{CO}_{3}^{*}$ could explain this discrepancy.

IV.8. Summary and Conclusions

In summary, to explain the observed profiles of $\delta^{13} \mathrm{C}-\left[\mathrm{CO}_{2}\right.$ and $\sum \mathrm{CO}_{2}$ in pore waters of Buzzards Bay, it has been proposed that: 
Figure IV.6. A diagram of the relative concentration of carbonate species as a function of $\mathrm{pH}$, assuming $\left[\mathrm{CO}_{2}=1.0 \mathrm{mM}\right.$ (adapted from Stumm and Morgan, 1981). 
Figure IV. 6

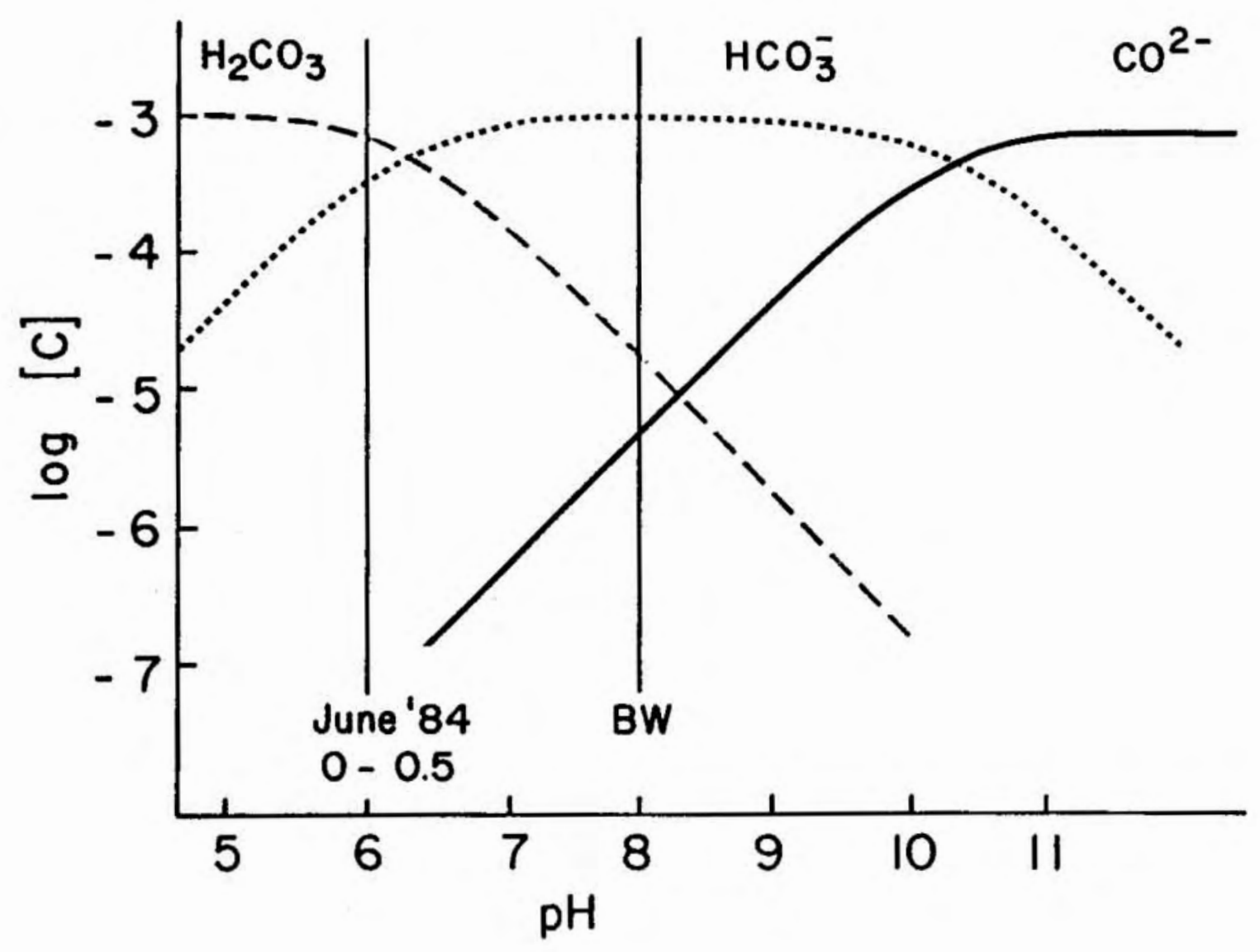


1) The $\delta^{13} \mathrm{C}$ of the $\mathrm{CO}_{2}$ added due to the oxidation of $\mathrm{SOC}$ is approximately $30 / 00$ greater than the $\delta^{13} \mathrm{C}$ of SOC, and

2) there is a significant flux of carbonate ion from bottom water to pore water, from $3-27 \%$ of the total flux of $\left[\mathrm{CO}_{2}\right.$ across the sediment water interface.

In the carbon budget constructed in Chapter III, it was considered that the $\left[\mathrm{CO}_{2}\right.$ added to the pore waters from bottom water was zero. If the flux of $\left[\mathrm{CO}_{2}\right.$ consists of recycled bottom water carbonate ion, then the value of the total amount of carbon oxidized in the sediments annually ( $\sum C_{0 x}$, Section III. . ) must be adjusted to reflect this. The correction involves a maximum of up to $27 \%$ of the $\Sigma \mathrm{CO}_{2}$ added originating from bottom water and is:

$\sum C_{\text {oxoorr }}=\sum C_{0 \times}(1-.27)=65 \mathrm{gC} / \mathrm{m}^{2}-\mathrm{yr}$.

This study indicates that the $\delta^{13} \mathrm{C}$ of the carbon oxidized in these sediments is not representative of the $\delta^{13} \mathrm{C}$ of the carbon present in the sediments. However, it is certain that further work is needed on this problem. The actual value of $\delta^{13} \mathrm{C}_{\text {org }}$ needs to be better substantiated. More incubation experiments would be one way to accomplish this. It would also be useful to investigate the $\delta^{13} \mathrm{C}$ of $D O C$ in pore waters. It is possible that the $\delta^{13} \mathrm{C}$ of $D O C$ will reflect the fractionation during oxidation proposed here.

It will also be important to investigate what causes this fractionation and to document whether it is important elsewhere in the marine environment. If the observed fractionation is due to the complex sources of carbon to coastal sediments, then it may be unimportant in oceanic sediments. However, 
if the fractionation is due to a molecular process, such as the preferential cleavage of carboxyl groups, it could be a very important process in oceanic sediments. 
Chapter V

\section{The Sources of Organic Carbon to the Sediments}

of Buzzards Bay

\section{V.1 Introduction}

There are three significant sources of organic carbon to coastal systems. Carbon fixed during photosynthesis by phytoplankton is a source of fresh, readily metabolizable organic matter. Other sources of organic matter are terrestrial carbon, supplied through run-off or riverine transport, and grasses produced in the extensive salt marshes adjacent to many coastal waters are a source of organic matter. Several authors have attempted to determine the relative supply of these three sources to coastal waters and sediments. However, this has proven difficult to determine.

Whether salt marsh grasses are a source of carbon to coastal sediments is a matter of debate. Valiela et al. (1982) found that $20 \%$ of the net annual above-ground production of Great Sippewissett Marsh was exported into Buzzards Bay. Using lignin analyses, Wilson et al. (1985) estimated that up to $30 \%$ of the vascular plant debris in Buzzards Bay sediments was from salt marsh grasses (Spartina sp.) and suggested that the remainder was from the eel grass, Zostera marina. In a study of stable carbon isotopes in a Georgia coastal system, Sherr (1982) decided that salt marsh carbon was not an 
important source of carbon to coastal sediments. However, because the range of $\delta^{13} \mathrm{C}$ values for the source materials is so large and because the possibility of alteration exists (Peterson et al., 1980), the relative contribution of organic carbon from the sources listed above cannot be determined unambiguously from stable carbon isotope evidence alone.

In this chapter, stable carbon and natural radiocarbon isotope ratios will be used to determine the sources of organic carbon to Buzzards Bay sediments. Additionally, pyrolysis-GCMS data will be used to provide further information on the sources.

\section{V.2. $\delta^{13} \mathrm{C}$ and $\Delta^{14} \mathrm{C}$ Results}

In order to assess the potential importance of different sources of organic carbon to Buzzards Bay, natural concentrations of ${ }^{13} \mathrm{C}$ and ${ }^{14} \mathrm{C}$ were measured in the sediments of the bay, plants, and soils in the surrounding area. Table V.I shows these data grouped according to their location - land, salt marsh, or bay. The $\delta^{13} \mathrm{C}$ values of the sources range from $-100 / 00$ to $-270 / 00$ while the sediment has values $(-21 \pm 10 / 00)$ intermediate in this range. The $\Delta^{14} \mathrm{C}$ values range from +100 to $-3100 / 00 ;$ all the sediment organic carbon samples are depleted, while all the source samples are enriched in ${ }^{14} \mathrm{C}$. Literature values of $\Delta^{14} \mathrm{C}$ and radiocarbon ages pertinent to this study are listed in Table V.2. The $\delta^{13} \mathrm{C}-\mathrm{SOC}$ measured in the core taken for ${ }^{14} \mathrm{C}$ analyses is 0.4 to 1.4 o/oo less than the average value obtained for $\delta^{13} \mathrm{C}-\mathrm{SOC}$ at the sampling site. The radiocarbon core was taken at a site within one kilometer of the array of buoys which defined the sampling site. The exact site was not sampled because radioactive isotopes 
Table V.1. Measured values of $\delta^{13} \mathrm{C}$ and $\Delta^{14} \mathrm{C}( \pm 1$ s.d.) for Buzzards Bay sediments and possible sources of organic carbon.

Sample

$\Delta^{14} \underline{C}(0 / 00)$

$\delta^{13} \underline{C}(0 / 00)$

Sediment

sfc. floc

$$
-31(20)
$$

$0-6 \mathrm{~cm}$

-155 (11)

-149 (20)

$-21.0$

13-16

29-32

61-64

-186 (11)

$-$

$-248(10)$

$-22.0$

102-106

$-313(10)$

$-22.0$

$-21.0$

Sediment

$0-30$

-

$-20.6$

Marine

plankton

+62 *

Zostera

$+62(6)$

$-17.7^{\star *}$

$-10.1$

Terrestrial

soil

$+104(9)$

$-26.7$

Salt marsh

$\begin{array}{lll}\text { Spartina } & +198(7) & -12.5 \\ \text { creek floc } & +107(9) & -16.5\end{array}$

*The $\Delta^{14} \mathrm{C}$ value for plankton is assumed to be the same as that for Zostera because they should both reflect the $\Delta^{14} \mathrm{C}$ of $\sum \mathrm{CO}_{2}$ (Penhale and Vace, 1980; Williams and Linick, 1975).

${ }^{* *}$ The $\delta^{13} \mathrm{C}$ of plankton was determined on material collected during a plankton tow in Buzzards Bay. The material was not completely planktonic as zooplankton and resuspended sediment were present in the sample. 
Table V.2. Literature values of $\delta^{13} \mathrm{C}$ and $\Delta^{14} \mathrm{C}$ for various carbon reservoirs.

\begin{tabular}{|c|c|c|c|c|c|}
\hline$\underline{\text { Sample }}$ & $\begin{array}{l}\Delta^{14} \mathrm{C} \\
(0 / 00)\end{array}$ & $\frac{\text { Age }}{\text { (yrs. B.P.) }}$ & $\begin{array}{l}\delta^{13} \mathrm{C} \\
(0 / 00)\end{array}$ & Location & Ref. \\
\hline zooplankton & & $-1030 \pm 80$ & & $\begin{array}{l}\text { Long Is land } \\
\text { Sound }\end{array}$ & 1 \\
\hline zooplankton & & & -18 to -22 & $\begin{array}{l}\text { Narragansett } \\
\text { Bay }\end{array}$ & 2 \\
\hline sewage sludge & & $-2980 \pm 70$ & -27 & $\begin{array}{l}\text { Long I S land } \\
\text { Sound }\end{array}$ & 1 \\
\hline fossil fuel & -1000 & $\infty$ & -27 & & 4 \\
\hline $\begin{array}{l}\mathrm{CO}_{2}, \quad 1985 \\
\text { atmosphere }\end{array}$ & +203 & & -8 & Heidelberg & 3,5 \\
\hline $\begin{array}{l}\mathrm{COO}_{2} \\
1984\end{array}$ & +96 & & & $\begin{array}{l}\text { Long Is I and } \\
\text { Sound }\end{array}$ & 6 \\
\hline coarse SPOM* & +227 & & -27 & Amazon & 7 \\
\hline fine SPOM & +19 & & -27 & Amazon & 7 \\
\hline
\end{tabular}

\begin{tabular}{ll}
\hline References: & 1. Benoit et al. (1979) \\
& 2. Gearing et al. (1984) \\
& 3. Levin et al. (1985) \\
& 4. Craig (1957) \\
& 5. Keeling et al. (1985) \\
& 6. Tanaka and Monaghan (1984) \\
& 7. Hedges et a1. (1986)
\end{tabular}

*SPOM refers to suspended particulate organic matter. 
had been released to the sediments within the array for other research projects. The site actually sampled was in the same depth of water (15 m), but the sediments were visibly sandier than those at the usual sampling site. However, it is unlikely that the radiocarbon levels in this core are substantially different from those at the sampling site since the sediments were still primarily silty-clays.

The $\Delta^{14} \mathrm{C}$ measured in the sediment decreases from $-1510 / 00(0$ to $6 \mathrm{~cm})$ to -313 o/00 (102 to $106 \mathrm{~cm}$ ) (Figure V.1). A sedimentation rate of $0.05 \mathrm{~cm} / \mathrm{yr}$ is implied from this radiocarbon distribution (Section V.4), which appears to be minimally affected by bomb ${ }^{14} \mathrm{C}$. The surface sediment floc, however, has a $\Delta^{14} \mathrm{C}$ value of $-310 / 00$ and clearly suggests the presence of bomb ${ }^{14} \mathrm{C}$. v.3. Pyrolysis-GCMS Results

Gas chromatograms obtained from pyrolysis-GC of the source samples are presented in Figure V.2a to d. Peak identifications were made by GC mass spectrometry. Peak identifications were made by comparison to 31,000 spectra contained in the computer data base reference library, not by running authentic standards, and thus the actual identifications must be considered tentative. However, the class of compound identified, e.g. a methoxyphenol, is more certain. The chromatograms shown in figure V.2a to $d$ are shown at the same attenuation $(x 16)$ for comparison. Ten $g$ of each sample were run although the $\% O C$ of each sample was very different (salt marsh sediment floc, $14 \%$; terrestrial, $4 \%$; sediment, $2 \%$ ). Because the salt marsh sample was so rich in pyrolyzable organic matter, many of the peaks at the beginning of the chromatogram were off scale. 
Figure V.1. Profile of $\Delta^{14} \mathrm{C}(0 / 00)$ with depth in the sediment. 
Figure V.I

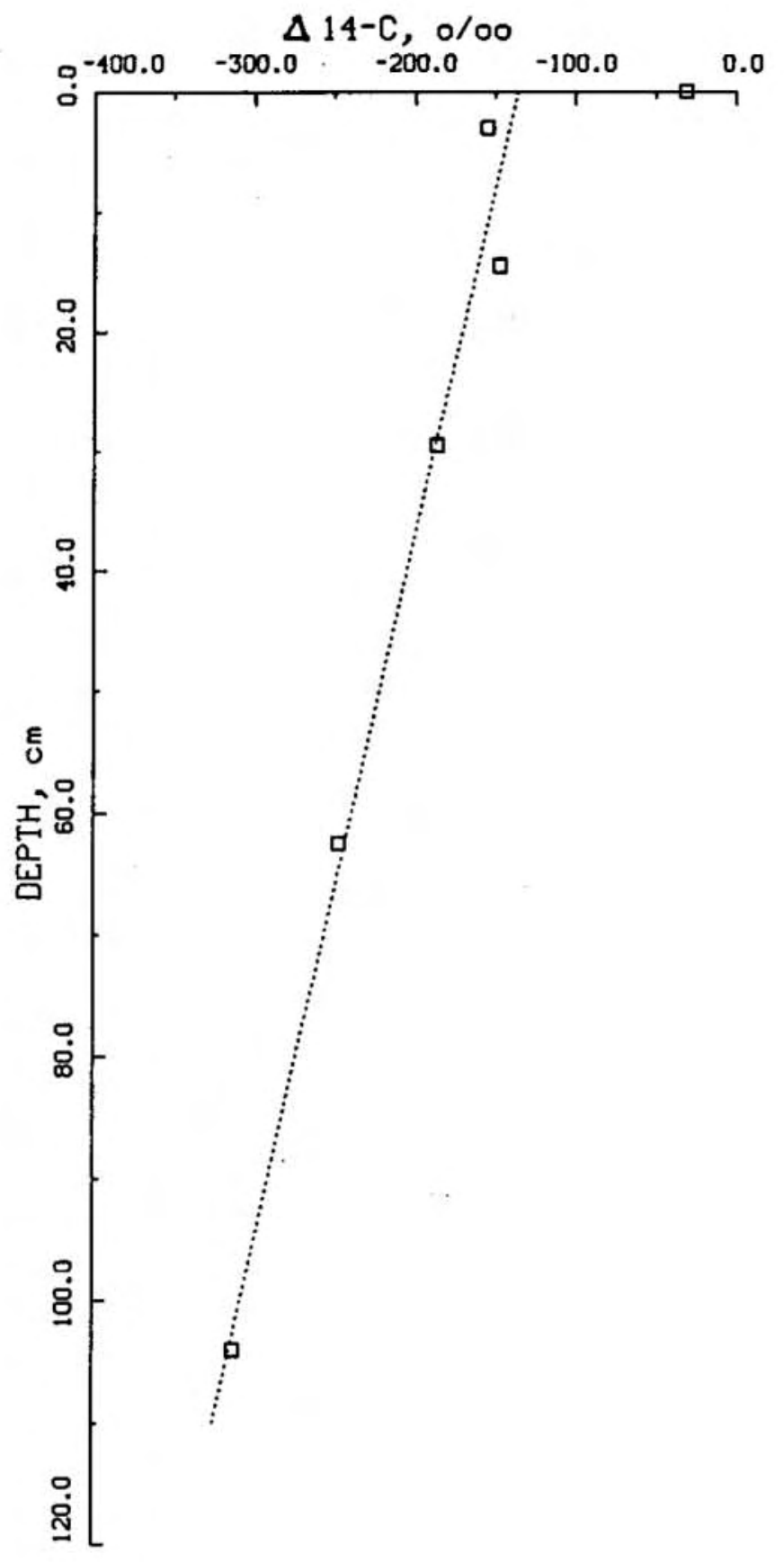

$-181-$ 
Figure V.2. Pyrolysis-GC chromatograms of soil, salt marsh, and sediment samples. 


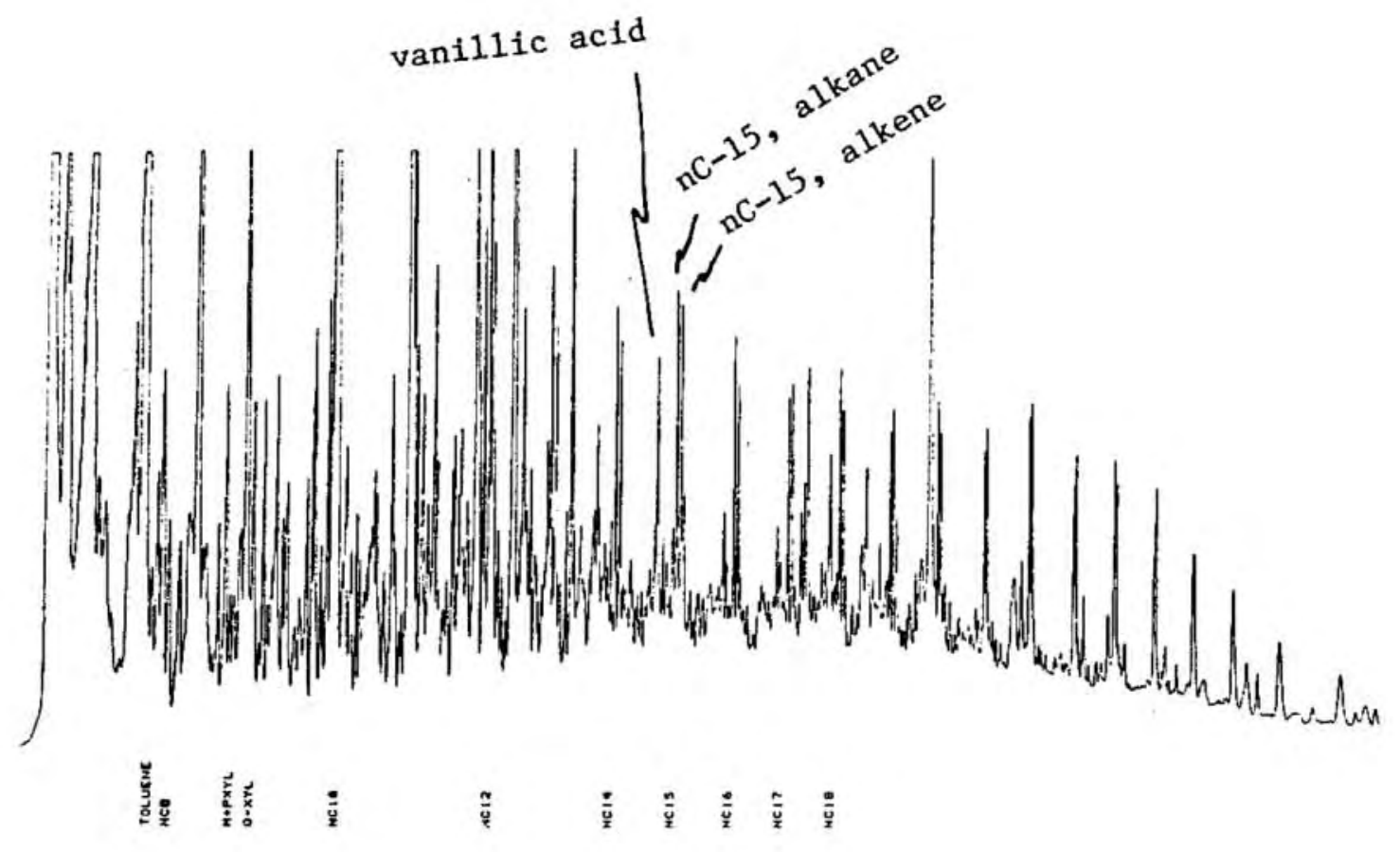

Figure V.2.a Salt marsh sediment floc

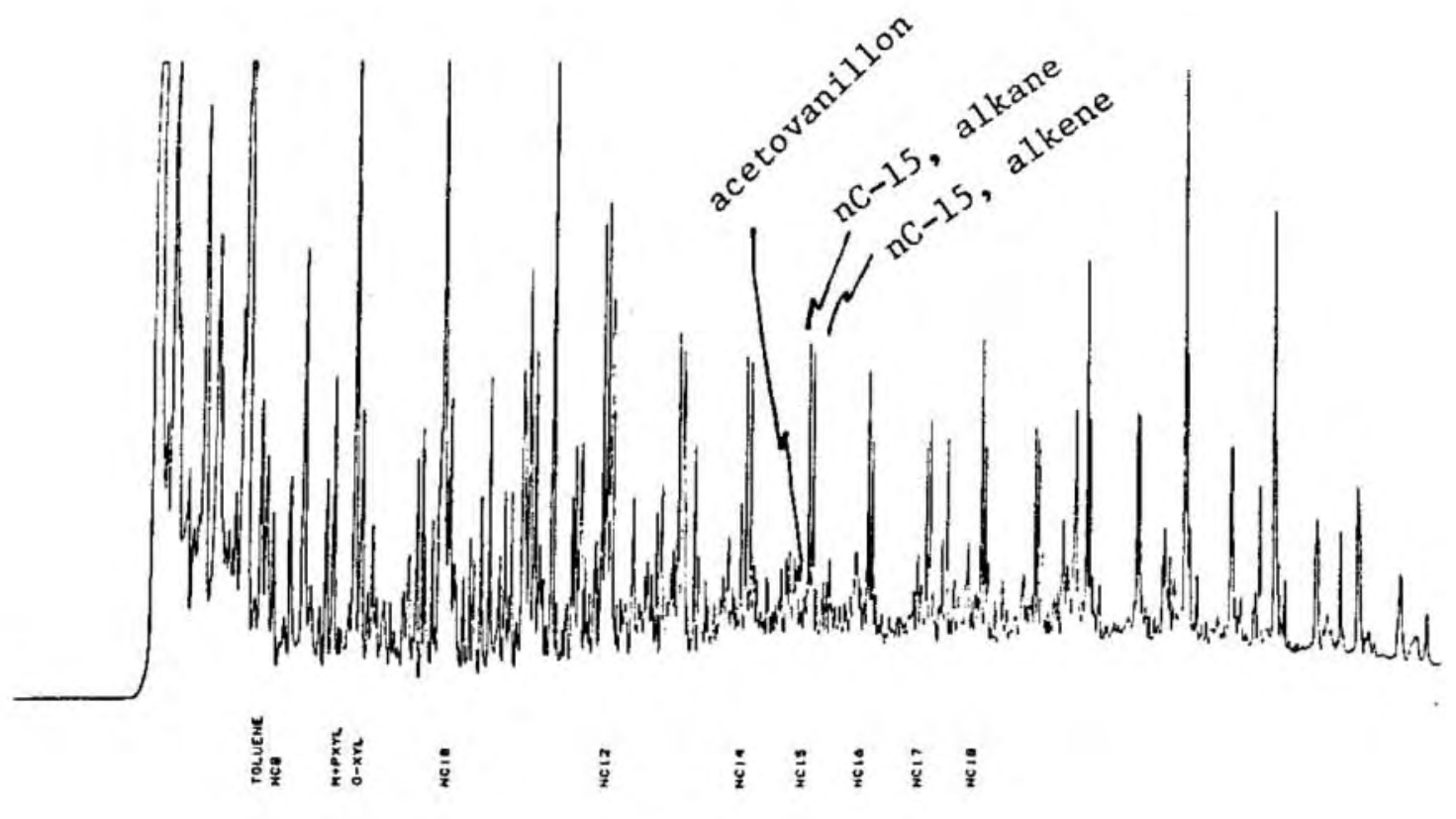

Figure V.2.b Terrestrial soil sample 


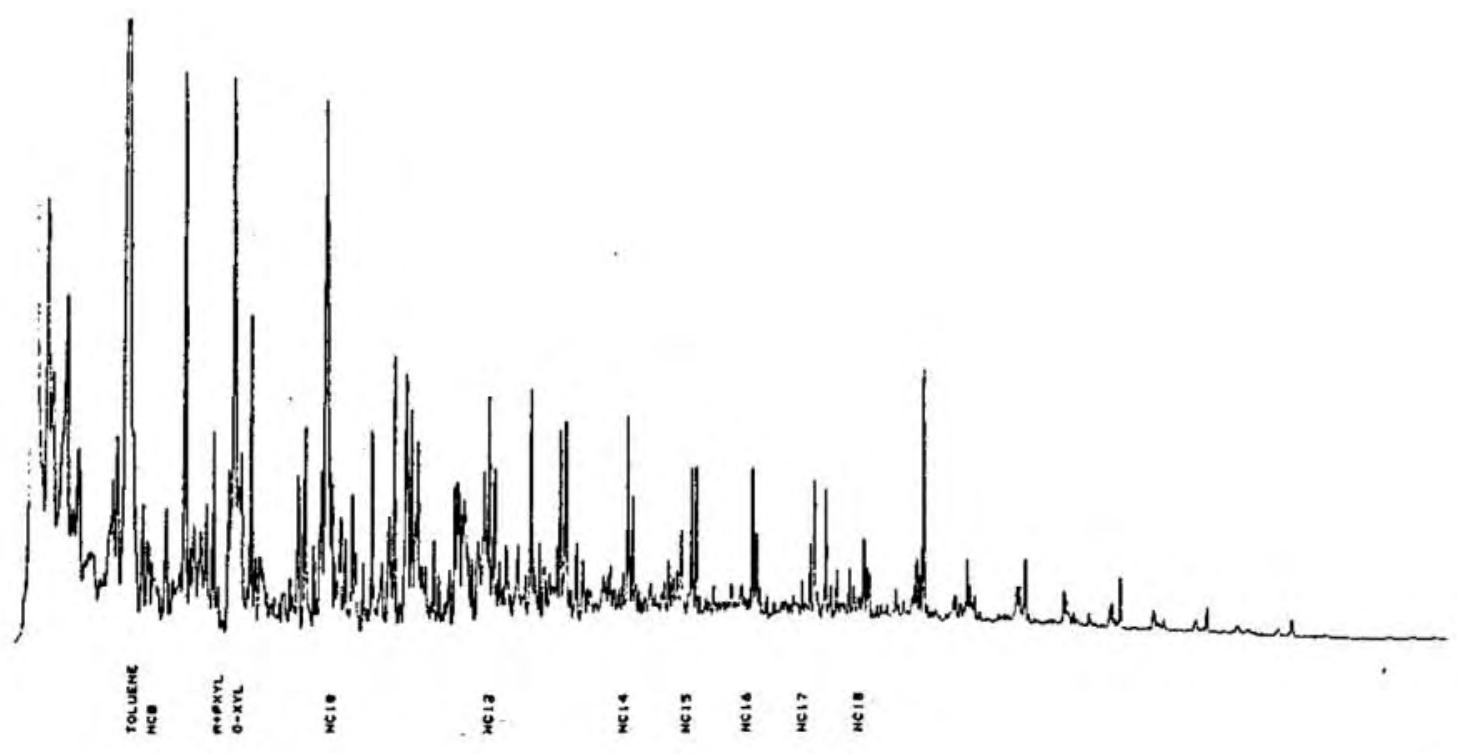

Figure V.2.c. Sediment sample, $0-0.5 \mathrm{~cm}$

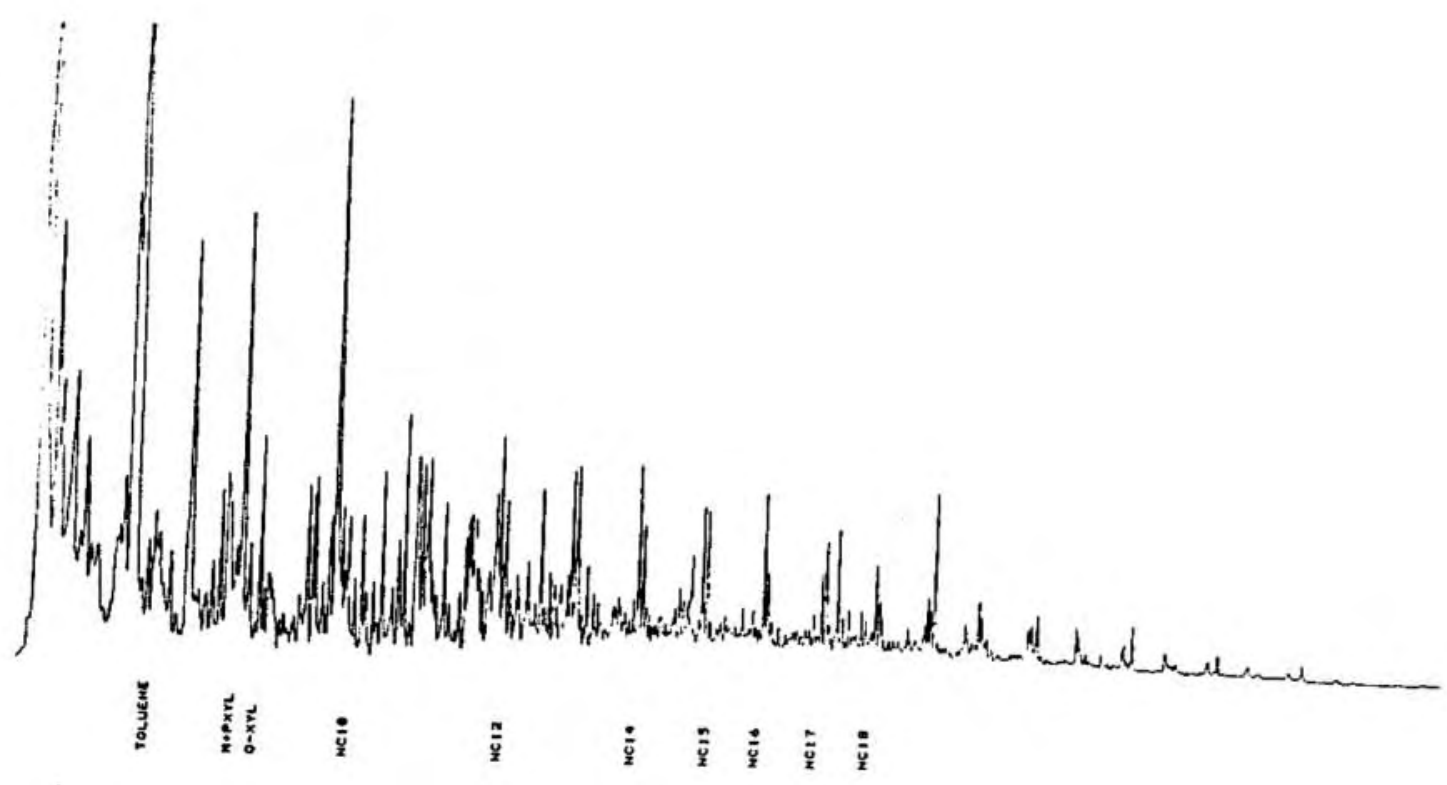

Figure V.2.d. Sediment sample, $5-6 \mathrm{~cm}$ 


\section{V.4. Discussion}

V.4.A. Calculation of surface sediment $\Delta^{14} \mathrm{C}$ values

Radiocarbon and stable carbon isotope data were collected in order to set limits on the relative contributions of terrestrial, salt marsh, and marine carbon to Buzzards Bay sediments. Using the radiocarbon data in Table V.l, it is possible to calculate the $\Delta^{14} \mathrm{C}$ of the carbon reaching the sediment water interface that is preserved in the sediments.

The mixing rate information obtained from modelling the excess $210 \mathrm{~Pb}$ profile (Section III.2.B.4) can be used to calculate $\Delta^{14} \mathrm{C}$ of the organic carbon reaching the sediment water interface by modelling the radiocarbon data assuming maximum mixing. A three-layer model (Martin, unpublished) can be used to interpret the radiocarbon data. In this model, it is assumed that there are two mixed zones above a zone affected by sedimentation only. Sedimentation and radioactive decay affect the profile throughout the sediment column. The curve was calculated using $z_{b}$, (the boundary between layers I and (I) $=3 \mathrm{~cm}, z_{b_{1} 1}=30 \mathrm{~cm}, D_{b_{1}}=30 \times 10^{-8} \mathrm{~cm}^{2} / \mathrm{sec}, D_{b_{2}}=10 \times$ $10^{-8} \mathrm{~cm}^{2} / \mathrm{sec}$. The values of these parameters were based on the information obtained from modelling the ${ }^{234} \mathrm{Th}$ (Martin, 1985) and ${ }^{210} \mathrm{~Pb}$ data. The deep ${ }^{14} \mathrm{C}$ data (> $30 \mathrm{~cm}$ ) were used to calculate $\omega(0.05 \mathrm{~cm} / \mathrm{yr}$ ) and the three layer model was used to determine that $C_{0}=11.3 \mathrm{cpm} / \mathrm{gdw}$. If it is assumed that this activity is well-mixed over $30 \mathrm{~cm}$, then, using the box model described in Bothner et a1. (1981), the $\Delta^{14} \mathrm{C}$ of the carbon reaching the seiment water interface is $-1100 / 00$ (Libby age* $=960 \mathrm{yrs}$ ). The large

* Libby age is defined in Chapter II. 
mixing coefficient, $D_{b}$, and mixing depth, $z_{b}$, used to obtain this fit indicate that there is extensive mixing in the sediments. This fit represents the maximum amount of mixing that might occur in the sediments.

It is possible that the extensive mixing indicated by excess ${ }^{234} \mathrm{Th}$ and $210 \mathrm{~Pb}$ data is the result of increased anthropogenic activity in recent times. Thus, the $\Delta^{14} \mathrm{C}$ profile at depth in the sediments may not be coupled with the ${ }^{234} \mathrm{Th}$ and ${ }^{210} \mathrm{~Pb}$ profiles. A minimum value of $\Delta^{14} \mathrm{C}$ can be obtained by assuming that there is no mixing in the sediments and extrapolating a least squares fit of the $\Delta^{14} \mathrm{C}$ data between 29 and $106 \mathrm{~cm}$ to the surface (Figure V.1). The data above $29 \mathrm{~cm}$ are not used in this $\mathrm{fit}$ because they may have been affected by recent events discussed below. The value of $\Delta^{\prime 4} \mathrm{C}$ calculated for the surface sediment is $-130 \mathrm{o} / 00$ (Libby age $=1120$ yrs).

The calculated minimum and maximum values of $\Delta^{14} \mathrm{C}$ at the surface $(-110$ and $-130 \mathrm{o} / 00$ ) are close to each other, but differ from the values actually measured at the surface of the sediment. This is most likely because the measured values at the surface reflect events of the past century while the calculated values are based on data unaffected by recent events. The $\Delta^{14} \mathrm{C}$ observed at the surface is the result of a complex set of events affecting the activity of atmospheric $\mathrm{CO}_{2}$ and organic carbon supplied to the marine environment over the past century. With the start of the Industrial Revolution, $\mathrm{CO}_{2}$ and organic carbon with no ${ }^{14} \mathrm{C}$ were added to the environment as the result of the combustion of fossil carbon. In the late $1950^{\prime}$ 's and early 1960's, thermonuclear weapons testing added significant quantities of ${ }^{14} \mathrm{CO}_{2}$ to the atmosphere. The Buzzards Bay samples of the 
surface floc and and of the sediment interval between 0 and $6 \mathrm{~cm}$ may show the influence of fossil and bomb carbon.

The surface sediment floc is thought to be representative of the easily resuspended material and fresh organic carbon deposited in the sediments. The $\Delta^{14} \mathrm{C}$ value of $-310 / 00$ is significantly enriched in ${ }^{14} \mathrm{C}$ in comparison to the sediment data and depleted in comparison to the source data. Because the sample contains unknown amounts of resuspended sediment and the $\Delta^{14} \mathrm{C}$ of the easily resuspended material is not known, it is difficult to estimate the true $\Delta^{14} \mathrm{C}$ of the new material arriving at the sediment water interface. However, the $\Delta^{14} \mathrm{C}$ of the sample indicates that there is a significant source of recent carbon to the sediment water interface.

The calculated range of surface $\Delta^{14} \mathrm{C}$ values $(-110$ to $-130 \mathrm{o} / 00)$ should represent the $\Delta^{14} \mathrm{C}$ of that portion of the carbon arriving at the sediment water interface which is preserved in the sediments. The carbon that actually arrives at the interface consists of carbon that will be preserved and carbon that will be oxidized in the sediments. The range of values is that which would be expected prior to the introduction of fossil and bomb carbon to the environment. The Libby age between 960 and 1120 years B.P. is significantly younger than the extrapolated age of 2320 years reported for Long Is land Sound sediments (Benoit et al. 1979). Since the sedimentation rates are similar, this suggests that either the relative supplies of terrestrial, salt marsh, and planktonic carbon are different or that the ${ }^{14} \mathrm{C}$ ages of the carbon sources are different in Long Island Sound than in Buzzards Bay.

V.4.B. Limits on Relative Contribution of Different Sources

By combining $\delta^{13} \mathrm{C}$ with $\Delta^{14} \mathrm{C}$ data, it is possible to set some 
limits on the relative amounts of terrestrial, salt marsh, and planktonic carbon preserved in the sediments of Buzzards Bay and on the ${ }^{14} \mathrm{C}$ signature of the carbon coming from salt marsh and terrestrial sources. The average $\delta^{13} \mathrm{C}-\mathrm{SOC}(-21.5 \mathrm{O} / 00)$ and $\Delta^{14} \mathrm{C}(-120 \mathrm{O} / 00)$ values for the core used for radiocarbon data reflect the combined $\delta^{13} \mathrm{C}$ and $\Delta^{14} \mathrm{C}$ values of the sources. The simultaneous solution of three mass balances can be used to evaluate the relative inportance of the three sources. The mass balances are:

$$
\begin{aligned}
& f_{p}+f_{t}+f_{s m}=1.0 \\
& \delta^{13} C_{s e d}=f_{p} \delta^{13} C_{p}+f_{t} \delta^{13} C_{t}+f_{s m} \delta^{13} C_{s m} \\
& \Delta^{14} C_{s e d}=f_{p} \Delta^{14} C_{p}+f_{t} \Delta^{14} C_{t}+f_{s m} \Delta^{14} C_{s m}
\end{aligned}
$$

where the subscripts sed, $p, t$, and sm refer to sediment carbon, planktonic carbon, terrestrial carbon, and salt marsh carbon, respectively. Ideally, the only unknowns in this equation are the relative proportions of planktonic, terrestrial, and salt marsh materials.

It is possible to assign $\delta^{13} \mathrm{C}$ values to all the carbon pools and $\Delta{ }^{14} \mathrm{C}$ values to all but the terrestrial carbon. The isotope values are based on measurements made in this study. A $\delta^{13} \mathrm{C}$ value of $-270 / 00$ was used as the terrestrial end member, $-120 / 00$ as the salt marsh value, and -19 $0 / 00$ as the plankton value. For the purposes of this discussion, the contribution of Zostera $\left(\delta^{13} \mathrm{C}=-10 \mathrm{o} / 00\right)$ to marine organic carbon will not be considered. If Zostera is an important source of carbon to the sediments, the effect of this assumption will be to decrease the calculated amount of terrestrial carbon added to the sediments, i.e. the calculated $f_{\text {c }}$ is a minimum value. The $\delta^{13} \mathrm{C}$ of Spartina is used to represent the organic carbon that enters the bay from salt marshes. 
A value of $00 / 00$ is used for the $\Delta^{14} \mathrm{C}$ of Spartina, based on the fact that Spartina collected in this study reflects the $\Delta^{14} \mathrm{C}$ of the atmosphere (Table V.1). A value of $-500 / 00$ is assumed for plankton, since that is the value of pre-bomb, preanthropogenic $\Delta^{14} \mathrm{C}$ of seawater $\sum \mathrm{CO}_{2}$ (Druffel and Linick, 1978; Williams and Linick, 1975). This value is lower than that in the atmosphere $(0 \mathrm{o} / 00)$ due mainly to communication of surface waters with ${ }^{14} \mathrm{C}$ depleted subsurface waters (Broecker, 1980; Druffel and Suess, 1983). Again, the contribution of carbon from zostera is not considered. However, the $\Delta^{14} \mathrm{C}$ of zostera and plankton should be the same because they both use carbon from seawater.

The only value which cannot be well-constrained is the $\Delta^{14} \mathrm{C}$ of terrestrial carbon. However, because the $\Delta^{14} \mathrm{C}$ of the carbon arriving at the sediment water interface is $-120 \mathrm{o} / 00$, the terrestrial carbon must be depleted in ${ }^{14} \mathrm{C}$, i.e. very old. This is consistent with studies in Europe and Canada (Scharpenseel et al., 1969; Campbell et al., 1967) which have shown that soil organic carbon is old. The terrestrial sample collected in this study (Table V.1) contained a significant amount of bomb-produced ${ }^{1}{ }^{4} \mathrm{C}$ and is not representative of pre-bomb soil organic matter. A recent study of particulate and dissolved organic matter in the Amazon River (Hedges et al., 1986) showed that the fine particulate organic matter $\left(\Delta^{\prime 4} C=190 / 00\right)$ was significantly depleted in ${ }^{14} \mathrm{C}$ compared to the coarse particulate organic matter $\left(\Delta^{14} \mathrm{C}=227 \mathrm{o} / 00\right)$ and dissolved humics $\left(\Delta^{1{ }^{4} \mathrm{C}} \simeq 270 \mathrm{o} / 00\right)$. This observation supports the idea that some fraction of the organic carbon in soils may be old, and that this fraction of the organic carbon may be associated with fine-grained particles. The ${ }^{14} \mathrm{C}$ depleted carbon reaching 
the sediments of Buzzards Bay $\left(\Delta^{\prime 4} \mathrm{C}=-120 \mathrm{o} / 00\right)$ is due most likely to the transport of fine-grained material from terrestrial soils.

The above equations were solved three times, each time assuming a different value of $\Delta^{14} \mathrm{C}$ for terrestrial carbon. The results of the mass balance are shown in Table V.3. This table shows that 0 to $70 \%$ of the carbon is planktonic, 30 to $60 \%$ terrestrial, and 0 to $40 \%$ salt marsh derived. The Libby age of the terrestrial carbon must be between 1690 and 2530 years old. It should be noted that these results have been calculated using the $\delta^{13} \mathrm{C}$-SOC from the core collected for radiocarbon data. As mentioned above, the $\delta^{13} \mathrm{C}-\mathrm{SOC}(-21.5 \mathrm{o} / 00)$ of this core is approximately 1 o/oo less than the $\delta^{13} \mathrm{C}-\mathrm{SOC}$ of the sediment from the sampling site $(-20.60 / 00)$. This shift suggests that the influence of terrestrial carbon is greater in the core for radiocarbon analysis.

V.4.C. Organic Geochemical Evidence for Allochthonous Sources

It is important to note that the results of the mass balance calculations for ${ }^{13} \mathrm{C}$ and ${ }^{14} \mathrm{C}$ listed in Table V.3 are entirely dependent on the choice of end member $\delta^{13} \mathrm{C}$ and $\Delta^{14} \mathrm{C}$ values. Thus, additional evidence for the input of terrestrial or salt marsh carbon was sought. The chromatograms from pyrolysis analysis shown in Figures $V . l$ a to $d$ show that there are dramatic differences between the chromatograms of the sediments and those of the salt marsh and terrestrial samples. In the sediment samples the most obvious feature in the high molecular weight end of the chromatogram is a series of alkene/alkane doublets. These doublets are most pronounced between the carbon numbers 14 to 16 . Whelan et al. (1983) have shown that this is an important feature in chromatograms of pyrolyzed phytoplankton. While there appears to 
Table V.3. The relative contributions of pre-bomb plankton, salt marsh, and terrestrial carbon to sediment preserved in Buzzards Bay assuming different values of $\Delta^{i 4} C_{t}$. The values in parentheses indicate the $\delta^{13} \mathrm{C}$

assumed for the source material in $0 / 00$.

$\begin{array}{llll}\Delta^{14} \underline{C}_{\mathrm{t}} & \frac{f}{(p}_{(-19)} & \frac{f_{\mathrm{t}}}{(-27)} & \frac{f_{s m}}{(-12)} \\ -190 & .01 & .63 & .36 \\ -240 & .52 & .39 & .09 \\ -270 & .67 & .32 & .01\end{array}$


be a homologous series of alkene/alkane peaks in the terrestrial and salt marsh samples, the doublets extend to a much higher carbon number than 16 . Simoneit (1961) observed a greater predominance of lower carbon number compounds in marine plants than in land plants.

Another important difference between the sediment and terrestrial/salt marsh chromatograms is the presence of methoxyphenols. Based on comparison with reference spectra, vanillic acid is present in the salt marsh sample and acetovanilion is present in the terrestrial sample. The structures of these compounds are shown in Figure V.3. The presence of methoxyphenols in pyrolysis products is believed to be specific to lignin (Wilson et al., 1983); lignin is a substance produced only by vascular plants. Many terrestrial plants, Spartina, and Zostera are vascular plants, although lignins are absent from Zostera (Wilson et al., 1985). No methoxyphenols were evident in the chromatograms of Buzzards Bay sediments. The absence of this product suggests that fresh vascular plant material from Spartina or terrestrial plants is not present in Buzzards Bay sediments. This conclusion is consistent with that of Wilson et al. (1985) from a study of lignin oxidation products in Buzzards Bay sediments. They concluded that only 5 to $7 \%$ of the organic carbon in the top $10 \mathrm{~cm}$ originated from vascular plant remains.

Thus, the carbon isotope results are not in complete agreement with the pyrolysis and lignin oxidation results. However, it is possible that the organic geochemical techniques used to identify land material in Buzzards Bay sediments are inappropriate due to the nature of the terrestrial or salt marsh organic matter in the sediments. As mentioned above, it is likely that the old material preserved in Buzzards Bay sediments is terrestrial carbon that 
Figure V.3. The structures of vanillic acid and acetovanillon. 
Figure V.3
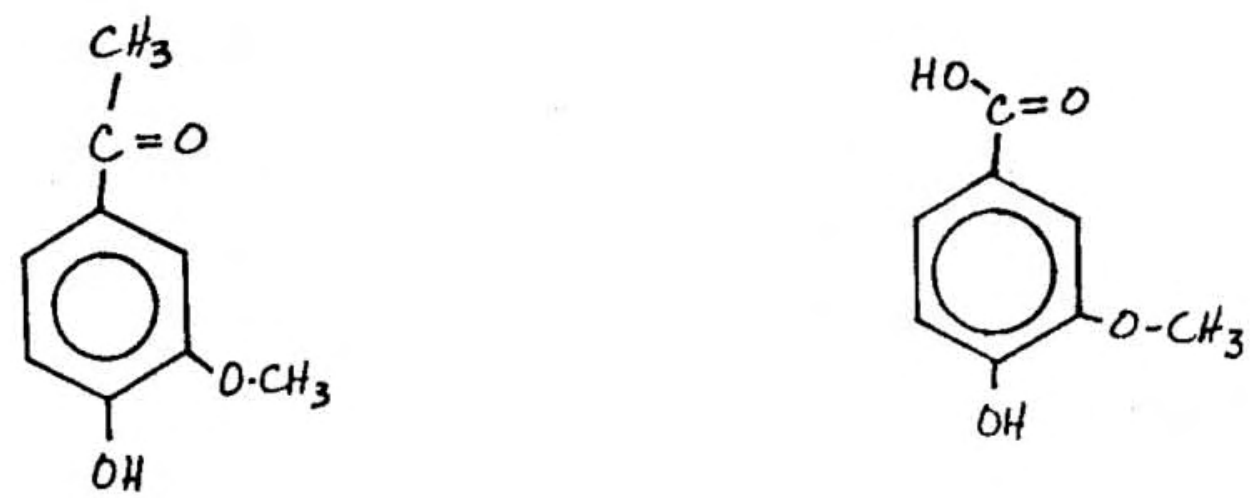

acteovanillon

vanillic acid 
was transported on fine-grained material and has been extensively degraded in the soils. The aerobic degradation of lignin results in a loss of methoxyphenols, and, thus, the specific terrestrial biomarkers for both CuO oxidation and pyrolysis. Ertel and Hedges (1984) have shown that lignin in soil can be modified beyond recognition by CuO oxidation, presumably from reaction with oxygen. Studies of fatty acids (Farrington et al., 1977) and polycyclic aromatic hydrocarbons (Hites et al., 1977) in Buzzards Bay sediments indicate that there is transport of material from land to the sediments, but do not provide a means of quantifying the allochthonous input.

The most compelling evidence that there is a significant source of carbon other than marine to the sediments is the $\Delta^{14} \mathrm{C}$ data. Based on a knowledge of the mixing in these sediments and considering the pre-bomb, pre-Industrial Revolution data, there is not enough mixing in the deep sediments to maintain the age of the sediments, unless there is a source of carbon that is significantly depleted in ${ }^{14} \mathrm{C}$ (i.e. very old) to the sediments. Thus, based primarily on the isotopic evidence, it appears that there is a significant contribution of allochthonous carbon to Buzzards Bay sediments. Evidence from the pyrolysis of sediments suggests that the contribution of non-marine carbon is small, and, thus, that the actual value of $f_{p}$, the relative contribution of pre-bomb plankton to the sediments is 0.67 (Table V. 3 ). Thus, almost all of the non-marine carbon supplied to these sediments is terrestrial and very old $\left(\Delta^{14} \mathrm{C}=-270 \mathrm{o} / 00\right.$, Libby age $\left.=2530 \mathrm{yrs}\right)$. 


\section{Chapter VI}

\section{SUMMARY AND CONCLUSIONS}

In this thesis, Buzzards Bay, a shallow Massachusetts coastal area was studied. The sediments in this bay are organic-rich, silty-clay sediments which become anoxic just below the surface. The concentration and stable carbon isotopic composition of $\mathrm{CO}_{2}$ in pore water were investigated as indicators of the seasonal rate of oxidation of organic carbon in coastal sediments. This study has shown that seasonal fluctuations in the observed profile of pore water $\sum \mathrm{CO}_{2}$ are directly related to seasonal changes in the remineralization rate of organic carbon and the extent of irrigation in nearshore sediments. The study of the isotopic data indicates that the oxidation of organic carbon is not the only important process affecting the $\delta^{13} \mathrm{C}-\sum \mathrm{CO}_{2}$.

VI.1. Remineralization of Organic Carbon

Pore water profiles of $\left[\mathrm{CO}_{2}\right.$ and $\delta^{13} \mathrm{C}-\left[\mathrm{CO}_{2}\right.$ respond directly to the oxidation of organic carbon to $\mathrm{CO}_{2}$. However, the concentrations of $\sum \mathrm{CO}_{2}$ and $\sum^{13} \mathrm{CO}_{2}$ have not been used to estimate remineralization rates of organic carbon in sediments before. By mathematically modelling the $\sum \mathrm{CO}_{2}$ depth profile, it was estimated that $67-85 \mathrm{gC} / \mathrm{m}^{2}$ are oxidized in the sediments annually and $5 \mathrm{gC} / \mathrm{m}^{2}-\mathrm{yr}$ are buried. The total amount of 
organic carbon that can be accounted for in the sediments, i.e. the amount oxidized plus the amount buried, was compared to the amount of primary productivity in Buzzards Bay reported by Roman and Tenore (1974). If their estimate is correct, $90 \%$ of the annual primary productivity is remineralized in the sediments. However, the study site was an area of fine-grained sediment, while Buzzards Bay has distinct regions of sandy sediments as well as fine-grained sediments. The remineralization of organic carbon is probably insignificant in sandy sediments. Then, the sediments carbon accounted for in this budget is $40 \%$ of the primary productivity. Recent estimates show that productivity can be as much as two times previous estimates by older techniques. If the reported primary productivity were low by a factor of two, then only $20 \%$ of the annual primary productivity would reach the sediments. The limitations of this estimate are the lack of knowledge about the spatial variability of remineralization rates in Buzzards Bay and the uncertainties in the primary production measurement. The seasonal, rather than the spatial, variability in oxidation rates was investigated in this study because temporal changes are an important factor in understanding the annual remineralization of organic carbon in coastal sediments.

In the warmer months of the year when most of the annual remineralization occurs in the sediments, the $\sum \mathrm{CO}_{2}$ profile was described well by a steady-state model. The model included irrigation, diffusion, and the oxidation of organic carbon to $\mathrm{CO}_{2}$ as the important processes affecting $\sum \mathrm{CO}_{2}$. The oxidation of organic carbon was described by a $\mathrm{CO}_{2}$ production term $\left(R_{c}\right)$ that was assumed to be constant to about $7 \mathrm{~cm}$ depth in the sediment column and to decrease exponentially below this. Implicit 
assumptions about this $\mathrm{CO}_{2}$ production term were that the rate of oxidation is related to the quality of organic matter avallable to the organisms in the sediments, rather than to the concentration of the electron acceptor being used, and that the surface sediments are well enough mixed for high quality organic matter to be available throughout the surface $0-7 \mathrm{~cm}$.

The values of $R_{c}$ calculated from this model for the surface sediment range from a low of $4.0 \times 10^{-9} \mathrm{~mol} / \mathrm{L}-\mathrm{sec}\left(294 \mathrm{nmol} / \mathrm{cm}^{3}\right.$-day) in October 83 to a high of $7.5 \times 10^{-9} \mathrm{~mol} / \mathrm{L}-\sec \left(551 \mathrm{nmol} / \mathrm{cm}^{3}\right.$-day) in August 84 . Values of $R_{c}$ at 10 and $20 \mathrm{~cm}$ are $3-13 \%$ and $0.1-2 \%$ of the surface remineralization rates, respectively. It was found that the model-predicted value of $R_{c}$ was dependent on the value chosen for the irrigation parameter, $\alpha$; if the irrigation parameter is known to $\pm 20 \%$, then the remineralization rates are known to this certainty also. This dependence emphasizes the importance of a knowledge of the mechanisms and magnitude of pore water transport in a nearshore environment when modelling pore water profiles. At this study site the enhanced transport of solutes had been well-characterized with ${ }^{22}{ }^{2} \mathrm{Rn}$ (Martin, 1985).

During colder months, the pore water profiles were not at steady-state concentrations. In these months, the rate of remineralization was slow and in two months, an event such as storm-induced irrigation, had reduced pore water concentrations to near bottom water values. It was possible to calculate a $\mathrm{CO}_{2}$ production term for only one of these months (December 83 ). The value of $R_{c}$ was $0.6 \times 10^{-9} \mathrm{~mol} / \mathrm{L}-\mathrm{sec}\left(45 \mathrm{nmol} / \mathrm{cm}^{3}\right.$-day), significantly lower than those calculated in the summer months.

The model-calculated values of $R_{c}$ are of the same order as sulfate 
reduction rates measured in nearshore sediments (Howarth, 1984; Westrich, 1985; and others). This indicates that the measured sulfate reduction rates are providing an accurate estimate of the remineralization occurring in coastal sediments. The discrepancies between $R_{c}$ and sulfate reduction rates are probably due to spatial heterogeneity in the sediments and to the fact that the ${ }^{35} \mathrm{SO}_{4}$ technique only measures the importance of one electron acceptor while $R_{c}$ responds to a suite of electron acceptors. Because the values of $R_{c}$ calculated in this study represent an average over a larger area of sediment than the sulfate reduction rates, $R_{c}$ is a better indicator of the actual remineralization occurring at a given site.

In addition to the solution model, an independent solid-phase model was developed to provide an estimate of the amount of carbon oxidized in the sediments. In this model, the profile of metabolizable organic carbon was explained by bioturbational mixing and oxidation of organic carbon. Solid-phase mixing rates at this site have been characterized with ${ }^{234} \mathrm{Th}$ (Martin, 1985), ${ }^{210} \mathrm{~Pb}$ (Brownawel1, 1986; this study), and ${ }^{14} \mathrm{C}$ (this study). In general, the oxidation rate in the surface sediments was found to be much greater in the summer months of June and August than in the other months studied. This is in agreement with the results from the solution model. However, the amount of carbon oxidized in the sediments calculated for each month using the solid-phase model differed unsystematically from that calculated with the solution model by as much as a factor of five. The solution model results are more accurate because the dissolved phase more rapidly reflects the temporal changes observed in a coastal environment. A study of the approach to steady-state showed that the solution-phase data 
start to approximate the steady-state profile, even given a drastic initial condition, after 2-4 weeks. The slower mixing rates of solid-phase species indicate that the approach to steady-state will take months. Thus, in environments which change seasonally and respond to specific events (e.g. storms), it is essential to consider the solution phase as well as solid-phase data in considering sediment cycling of carbon.

Thus, this study has shown that accurate remineralization rates can be calculated from the seasonal profiles of $\mathrm{CO}_{2}$ in irrigated sediments. An accurate description of the $\mathrm{SCO}_{2}$ depth profile in the sediments was essential for the interpretation of the profile of $\delta^{13} \mathrm{C}-\left[\mathrm{CO}_{2}\right.$. VI.2. Sources of Carbon to Pore Water $\sum_{\mathrm{CO}_{2}}$

The isotopic signature of $\sum \mathrm{CO}_{2}$ was used as an indicator of the processes affecting $\mathrm{CCO}_{2}$ in pore waters. During every month studied, the oxidation of organic carbon to $\mathrm{CO}_{2}$ provided a significant amount of carbon to the $\left[\mathrm{CO}_{2}\right.$ pool. However, in every month, the $\delta^{13} \mathrm{C}$ of $\sum \mathrm{CO}_{2}$ added to the pore water in the surface sediments was enriched in ${ }^{13} \mathrm{C}$ with respect to the $\delta^{13} \mathrm{C}$ of SOC. Using the steady-state solution model used to describe the $\left[\mathrm{CO}_{2}\right.$ data, the $\delta^{13} \mathrm{C}$ of $\sum \mathrm{CO}_{2}$ added to the pore water in the surface sediments had to be greater than $-15 \% / 00$. This value is significantly greater than the $\delta^{13} \mathrm{C}$ of $\operatorname{SOC}(-20.6 \mathrm{o} / 00)$. In most months, the $\delta^{13} \mathrm{C}$ of $\left[\mathrm{CO}_{2}\right.$ added to the pore water in the sediments deeper than $7 \mathrm{~cm}$ was between -20 and $-210 / 00$. This suggests that either the $\delta^{13} \mathrm{C}$ of $\mathrm{CO}_{2}$ produced from the oxidation of organic carbon is different from the $\delta^{13} \mathrm{C}$ of organic carbon in the surface sediments or that there is an additional source of ${ }^{13} \mathrm{C}$-enriched carbon to the pore waters. 
The dissolution of $\mathrm{CaCO}_{3}\left(\delta^{13} \mathrm{C}=1.7 \mathrm{o} / 00\right)$ is one possible source of ${ }^{13} \mathrm{C}$-enriched carbon to the pore waters. Dissolved Ca profiles indicated that there was significant dissolution of $\mathrm{CaCO}_{3}$ in nearshore sediments during the spring months. In June 84 and March 85, dissolved $\mathrm{Ca}$ concentrations were increased over bottom water concentration by as much as $20 \%$. The springtime $\mathrm{Ca}$ concentrations suggest an annual dissolution of as much as $4.2 \mathrm{~g} \mathrm{CaCO}_{3} / \mathrm{m}^{2}$. Acid was produced throughout the year by the oxidation of organic carbon by oxygen. A second source of acid was the oxidation of solid-phase sulfides; this process appears to have been especially important in the spring. In August 84, the pore waters between 0 and $4 \mathrm{~cm}$ were significantly undersaturated with respect to $\mathrm{CaCO}_{3}$, yet the dissolved $\mathrm{Ca}$ profile did not indicate that there was dissolution of $\mathrm{CaCO}_{3}$. In this month, there was no $\mathrm{CaCO}_{3}$ present in the sediments to dissolve. However, the dissolution of $\mathrm{CaCO}_{3}$ cannot account entirely for the observed enrichment of ${ }^{13} \mathrm{C}$ in $\sum \mathrm{CO}_{2}$ added to the pore water.

An additional source of ${ }^{13} \mathrm{C}$-enriched carbon was bottom water carbonate ion. In every month studied, there was a net flux of $\sum \mathrm{CO}_{2}$ from pore water to bottom water. The flux of pore water $\sum \mathrm{CO}_{2}$ to bottom water $\left(\mathrm{J}_{t}\right)$ ranged from a minimum of $10 \times 10^{-12} \mathrm{~mol} / \mathrm{cm}^{2}-\mathrm{sec}$ in December 83 to a maximum of $50 \times 10^{-12} \mathrm{~mol} / \mathrm{cm}^{2}-\mathrm{sec}$ in August 84 . However, the $\mathrm{pH}$ of bottom water was about 8 while that of the pore water was less than or equal to 7. Pore water was more acidic because of the addition of acid from the oxidation of organic carbon and solid-phase sulfides. Because of this, the relative proportion of the different species of inorganic carbon $\left(\mathrm{H}_{2} \mathrm{CO}_{3}^{\star}\right.$, $\mathrm{HCO}_{3}^{-}, \mathrm{CO}_{3}^{2-}$ ) was very different in bottom water and pore water; the 
concentration of $\mathrm{CO}_{3}^{2-}$ in bottom water was greater than the concentration in pore water. Thus, while there is a net flux of $\sum \mathrm{CO}_{2}$ from pore water to bottom water, there is a flux of carbonate ion from bottom water to pore water. Because bottom water $\sum \mathrm{CO}_{2}$ was more ${ }^{13} \mathrm{C}$-enriched than pore water $\sum \mathrm{CO}_{2}$, the transfer of bottom water carbonate ion to pore water was a source of ${ }^{13} \mathrm{C}$-enriched carbon to the pore water. If the $\delta^{13} \mathrm{C}$ of $\mathrm{CO}_{2}$ added to the pore water from the oxidation of organic carbon is $-20.6 \%$, then the flux of $\mathrm{CO}_{3}^{2-}$ from bottom water to pore water must be $10-30 \%$ of the total flux of $\left[\mathrm{CO}_{2}\right.$ from pore water to bottom water.

The influence of bottom water carbonate ion on pore water $\mathrm{COO}_{2}$ is maximized in a coastal environment. The flux of carbonate ion from bottom water is most important near the sediment water interface, but is important to greater depths because of the extent of irrigation. The flux of $\mathrm{CCO}_{2}$ across the sediment water interface due to irrigation can account for as much as $50 \%$ of the total flux. This is much higher than that calculated for dissolved metals (Martin, 1985), because there is no appreciable precipitation of carbonate solids in these sediments while there is significant metal precipitation. The estimate of the irrigational flux is limited by our knowledge of the permeability of burrow walls because the calculations assume that the transport of a solute across a burrow wall is similar to molecular diffusion across the sediment water interface. Since burrow linings probably inhibit diffusion (Aller, 1984), the irrigation estimates must be considered a maximum.

The diffusion of carbonate ion from bottom water to pore water can explain the enrichment of ${ }^{13} \mathrm{C}$ in pore water $\left[\mathrm{CO}_{2}\right.$ for every month except June 
84. In June 84 , the $\mathrm{SCO}_{2}$ in the pore waters between 0 and $3 \mathrm{~cm}$ was more enriched in ${ }^{13} \mathrm{C}$ than in any other month. The value of $\delta^{13} \mathrm{C}-\left[\mathrm{CO}_{2}\right.$ in the $0-0.5$ sediment interval $(+4.0 \mathrm{o} / 00)$ was more positive than the bottom water value $(+1.3 \mathrm{o} / 00)$ and than any potential source of carbon to the pore water identified in this study. The isotopic fractionation between the species of $\mathrm{SCO}_{2}$ coupled with the extremely low $\mathrm{pH}$ values observed in the pore waters between 0 and $3 \mathrm{~cm}$ suggested that diffusion of $\mathrm{H}_{2} \mathrm{CO}_{3}^{*}$ from the sediment might explain the observed isotope ratios. The flux of $\mathrm{COO}_{2}$ from the low pH pore water to bottom water contained a significant amount of $\mathrm{H}_{2} \mathrm{CO}_{3}^{*}$ (as much as $50 \%$ ). Since the isotopic fractionation between $\mathrm{H}_{2} \mathrm{CO}_{3}^{*}$ and $\mathrm{HCO}_{3}^{-}$is approximately 8 o/oo with $\mathrm{H}_{2} \mathrm{CO}_{3}^{*}$ enriched in ${ }^{12} \mathrm{C}$ (Deines, 1974), the June data suggest that diffusion of $\mathrm{H}_{2} \mathrm{CO}_{3}^{*}$ from the sediments removed significant quantities of ${ }^{12} \mathrm{C}$ from the sediments. VI.3. Nature of the Organic Carbon Oxidized

In response to the results from the field studies, laboratory experiments were conducted to determine whether the $\delta^{13} \mathrm{C}$ of $\mathrm{CO}_{2}$ produced from the oxidation of organic carbon $\left(\delta^{13} \mathrm{C}-O C_{0 x}\right)$ was different from the $\delta^{13} \mathrm{C}$ of organic carbon in the sediments $\left(\delta^{13} \mathrm{C}-\mathrm{SOC}\right)$. In the laboratory experiments, mud from the sampling site was incubated at a constant temperature. Three depths were studied $(0-3,10-15$, and $20-25 \mathrm{~cm})$. For the first study (IEI), sediment was stirred to homogenize it before packing into centrifuge tubes for incubation. For the second study (IE2), sediment was introduced directly into glass incubation tubes by subcoring. The second procedure greatly reduced disturbance to the sediment. Rates of $\mathrm{CO}_{2}$ production were calculated from the concentrations of $\mathrm{SCO}_{2}$ measured over 
up to 46 days.

In both studies, the values of $R_{c}$ in the deeper intervals were about $10 \%$ of the surface values. This is consistent with the results of the $\mathrm{ZCO}_{2}$ model, although the rates decrease more rapidly in the field. In all cases, the remineralization rates during the beginning of IEl were much greater than those at the beginning of IE2. The sediment for IEl was collected in February 84. The measured value of $R_{c}$ in the surface sediment of the laboratory experiment $\left(24 \times 10^{-9} \mathrm{~mol} / \mathrm{L}-\mathrm{sec}\right)$ is much greater than the value of $R_{c}$ observed in the field in another winter month, December $83\left(.62 \times 10^{-9}\right)$. The sediment for IE2 was collected in August 85 . The measured values of $R_{c}$ in the surface sediment $\left(6.6-12 \times 10^{-9} \mathrm{~mol} / \mathrm{L}-\mathrm{sec}\right)$ were consistent with the field values from August $84\left(7.5 \times 10^{-9}\right)$. The $\left[\mathrm{CO}_{2}\right.$ results indicate that IE2 reproduced field conditions more accurately than IEl did. This result stresses the importance of careful sediment sampling when studying microbial processes.

The isotopic results from the experiments strongly suggest that $\delta^{13} \mathrm{C}-\mathrm{OC} 0 \times$ in the surface sediments $(-17.80 / 00 \pm 1.90 / 00)$ is greater than $\delta^{13} \mathrm{C}-\mathrm{SOC}(-20.6 \pm 0.2 \mathrm{o} / 00)$. However, the variability in the experiments was great and further work must be done to confirm these results. The magnitude of the observed fractionation was small enough that the observed values of $\delta^{13} \mathrm{C}-\sum \mathrm{CO}_{2}$ in the pore waters can be explained by fractionated oxidation coupled with the diffusion of carbonate ion from bottom water to pore water.

Since $\delta^{13} \mathrm{C}-\mathrm{OC}_{0 \times}$ is greater than $\delta^{13} \mathrm{C}-\mathrm{SOC}$, the organic carbon preserved in the sediments is not representative of the carbon that is 
oxidized in the sediments. It appears that a selective portion of the organic matter is remineralized preferentially. This may happen at a variety of levels. Fresh phytodetritus is almost certainly oxidized first, because it is the most labile fraction of organic matter reaching the sediment water interface. It is possible that the $\delta^{13} \mathrm{C}$ of phytodetritus is different from that of the bulk organic carbon reaching the sediment water interface. It has been observed that certain groups of molecules are remineralized before the bulk organic carbon, e.g. Carbohydrates (Hatcher et a1. 1983), sterols (Lee et al., 1977), and amino acids (Henrichs, 1980). The preferential remineralization of carbohydrates could explain the observed fractionation if the concentration of carbohydrates in the oxidized organic carbon is greater than $50 \%$ because the $\delta^{13} \mathrm{C}$ of carbohydrates is $4-5 \mathrm{o} / 00$ enriched in ${ }^{13} \mathrm{C}$ over the bulk organic carbon. Likewise, preferential cleavage of carboxyl groups, which are enriched in ${ }^{13} \mathrm{C}$ would cause $\delta^{13} \mathrm{C}-\mathrm{OC}$ ox to be enriched in ${ }^{13} \mathrm{C}$. Further laboratory experiments are needed to confirm whether molecular-specific oxidation could explain the results of this study. VI.4. Conclusions

This study of the remineralization of organic carbon in Buzzards Bay showed that more than $90 \%$ of the organic carbon reaching the sediments is oxidized to $\mathrm{CO}_{2}$. To model the $\left\{\mathrm{CO}_{2}\right.$ profile, it was assumed that the remineralization occurs within the sediment column. The ability of the model to reproduce the observed profiles is one indicator that this assumption is valid. It is likely that remineralization rates are greatest near the sediment water interface, but that most of the remineralization occurs within the sediments. The rapid solid-phase mixing rates in nearshore sediments and 
the relatively short water column allow large quantities of easily metabolizable carbon to be well mixed into the sediment column. The increased diffusional area created by burrows enhances the supply of oxidants to the deeper sediments and provides avenues for the removal of potentially toxic metabolites. Thus, nearshore sediments represent an environment where the remineralization of organic carbon within the sediment column is maximized. While the ratio of organic carbon remineralized to organic carbon buried is similar in all sediment environments, the supply of organic carbon to coastal sediments is so large that proportionately more organic matter is remineralized in the sediments than compared to the open ocean (Bender and Heggie, 1984).

The $\delta^{13} \mathrm{C}$ of $\left[\mathrm{CO}_{2}\right.$ is a sensitive indicator of the processes affecting the dissolved inorganic pool. The pore water isotopic data indicate that there is a significant flux of carbonate ion from bottom water to pore water. Emerson and Bender (1981) discussed the importance of bottom water $\sum \mathrm{CO}_{2}$ and dissolution of $\mathrm{CaCO}_{3}$ as sources of $\mathrm{CO}_{3}^{2-}$ which could neutralize the acid formed from the oxidation of organic carbon by oxygen in deep sea sediments. Based on their estimates of the relative rates of oxidation and dissolution, they concluded that bottom water was an unimportant source of carbonate ion. However, the sediments they discussed were $\mathrm{CaCO}_{3}$-rich, unlike the coastal sediments studied here. In coastal sediments, the lack of $\mathrm{CaCO}_{3}$, the high quality of the organic matter, and the acid produced from the oxidation of organic matter and solid-phase sulfides create a situation where bottom water $\sum \mathrm{CO}_{2}$ is an important source of $\mathrm{CO}_{3}^{2-}$ to pore water.

The results of the incubation experiments strongly suggest that 
$\delta^{13} \mathrm{C}-O C_{0 \times}$ is greater than $\delta^{13} \mathrm{C}-\mathrm{SOC}$ and that this difference is greatest in the surface sediments. If this is so, then the difference between $\delta^{13} \mathrm{C}-O C_{0 \times}$ and $\delta^{13} \mathrm{C}-\mathrm{SOC}$ is most probably a result of the complex nature of the organic carbon reaching the sediment water interface in a coastal environment. In other words, the carbon oxidized first, and, thus, closest to the sediment surface, is fresh phytodetritus. The difference between the $\delta^{13} \mathrm{C}$ of phytodetritus and the $\delta^{13} \mathrm{C}$ of SOC cannot be seen in the sediments because of the rapid mixing rates in the upper $3 \mathrm{~cm}$.

\section{Future work}

It will be important for further research to document whether the organic carbon oxidized in the sediments is isotopically representative of the organic carbon in the sediments. The actual value of $\delta^{13} \mathrm{C}-\mathrm{OC} \mathrm{C}_{0 \times}$ needs to be verified. Further work with incubation experiments is one way to accomplish this. It is also important to investigate the $\delta^{13} \mathrm{C}$ of $\mathrm{DOC}$ in pore water as this carbon pool constitutes $10-25 \%$ of the total carbon in the dissolved phase. The $\delta^{13} \mathrm{C}$ of $D O C$ may reflect the fractionated oxidation suggested by this study.

It will also be important to investigate what causes fractionation during oxidation and to document whether it is important elsewhere in the marine environment. If the observed fractionation is due to the complex sources of carbon to coastal sediments, then it may be unimportant in open ocean sediments. However, if the fractionation is due to a molecular process, such as the preferential cleavage of carboxyl groups, it could be a very important process in oceanic sediments. 


\section{References}

Abelson, P.H., and T.C. Hoering (1961) Carbon isotope fractionation in formation of amino acids by photosynthetic organisms. PNAS $\underline{47}(5)$, 623-632.

Aller, R.C. (1977) The influence of macrobenthos on chemical diagenesis of marine sediments. Pho Thesis, Yale University, New Haven, CT.

Aller, R.C. (1980a) Diagenetic processes near the sediment water interface of Long Island Sound. I. Decomposition and nutrient element geochemistry. (S,N,P). Advances in Geophysics 22, 237-350.

Aller, R.C. (1980b) Quantifying solute distributions in the bioturbated zone of marine sediments by defining and average microenvironment. GCA 44, 1955-1965.

Aller, R.C. (1982) Carbonate dissolution in nearshore terrigenous muds: The role of physical and biological reworking. J. of Geol. 90, 79-95.

Aller, R.C., and J.Y. Yingst (1980) Relationships between microbial distributions and the anaerobic decomposition of organic matter in surface sediments of Long Island Sound, USA. Mar. Biol. 56, 29-42.

Alperin, M.J. and W.S. Reeburgh (1984) Geochemical observations supporting anaerobic methane oxidation. in Microbial Growth on $C_{1}$ Compounds, eds. R.L. Crawford and R.S. Hanson.

Behrens, E.W., and S.A. Frishman (1971) Stable carbon isotopes in blue-green algal mats. J. of Geol. $79,94-100$.

Bender, M.L. and D.T. Heggie (1974) Fate of organic carbon reaching the deep sea floor: a status report. GCA $\underline{48}$, 977-986.

Benninger, L.K., R.C. Aller, J.K. Cochran, and K.K. Turekian (1979) Effects of biological sediment mixing on the $210 \mathrm{pb}$ chronology and trace metal distribution in a Long Island Sound sediment core. EPSL 43, 241-259.

Benoit, G.J., K.K. Turekian, and L.K. Benninger (1979) Radiocarbon dating of a core from Long Island Sound. ECMS $\underline{9}, 171-180$.

Berner, R.A. and J.T. Westrich (1985) Bioturbation and the early diagenes is of carbon and sulfur. Am. Journ. of Sci. 285, 193-206.

Berner, R.A. (1980) Early Diagenesis: A Theoretical Approach, Princeton Univ. Press.

Berner, R.A. (1980) A rate model for organic matter decomposition during bacterial sulfate reduction in marine sediments. CNRS Publication 293, $35-44$. 
Bothner, M.H., E. Spiker, P.P. Johnson, R.R. Rendigs, and P.J. Aruscavage (1981) Geochemical evidence for modern sediment accumulation on the continental shelf off southern New England. Journ. of Sed. Petrol. 51, 281-292.

Boudreau, B.P. (1984) On the equivalence of nonlocal and radial-diffusion models for porewater irrigation. JMR 42, 731-735.

Brown, F.S., M.J. Baedecker, A. Nissenbaum, and I.R. Kaplan (1972) Early diagenesis in a reducing fjord, Saanich Inlet, B.C. III. Changes in organic constituents of sediment. GCA $\underline{36}, 1185-1203$.

Brownawell, B. (1986) The role of colloidal organic matter in the marine geochemistry of PCBs. PhO thesis, WHOI/MIT.

Campbe11, C.A., E.A. Paul, D.A. Rennie, and K.J. McCallum (1967) Applicability of the carbon dating method of analysis to soil humus studies. Sol1 Science 104, 217-224.

Claypool, G.E. and C.N. Threlkeld (1983) 9. Anoxic diagenes is and methane generation in sediments of the Blake Outer Ridge, DSDP site 533, Leg 76. Init. Repts. DSDP $\underline{76}, 391-402$.

Craig, H. (1957) Isotopic standards for carbon and oxygen and correction factors for mass spectrometric analysis of carbon dioxide. GCA 12 , 133-149.

Crank, J. (1975) The Mathematics of Diffusion, Second Edition. Oxford University Press.

Degens, E.T., R.R.L. Guillard, W.M. Sackett, and J.A. Hellebust (1968) Metabolic fractionation of carbon isotopes in marine plankton I. Temperature and respiration experiments. DSR 15, 1-9.

Degens, E.T., M. Behrendt, B. Gotthardt, and E. Reppmann (1968) Metabolic fractionation of carbon isotopes in marine plankton-II. Data on samples collected off the coasts of Peru and Ecuador. DSR 15, 11-20.

Degens, E.T. (1969) Biogeochemistry of stable carbon isotopes. in Organic Geochemistry, eds. G. Eglington and M.T.J. Murphy.

Deines, P. (1980) The isotopic composition of reduced organic carbon. in Handbook of Environmental Isotope Geochemistry. vol. 1, eds. P. Fritz and J. Fontes.

Deines, P., D. Langmuir, and R.S. Harmon (1974) Stable carbon isotope ratios and the existence of a gas phase in the evolution of carbonate ground waters. GCA $\underline{38}, 1147-1164$. 
Deuser, W.G., E.T. Degens, and R.R.L. Guillard (1968) Carbon isotope relationships between plankton and sea water. GCA $\underline{32}, 657-660$.

Deuser, W.G. (1970) Carbon-13 in Black Sea waters and implications for the origin of hydrogen sulfide. Science 168, 1575-1577.

Druffel, E.M. and H.E. Seuss (1983) On the radiocarbon record in banded coral: exchange parameters and net transport of ${ }^{14} \mathrm{CO}_{2}$ between atmosphere and surface ocean.

Druffel, E.M. and T.W. Linick (1978) Radiocarbon in annual coral rings of Florida. GRL $\underline{5}, 913-916$.

Emerson, S., R. Jahnke, M. Bender, P. Froelich, G. Klinkhammer, C. Bowser, and $G$. Setlock (1980) Early diagenesis in sediments from the eastern equatorial Pacific, I. Pore water nutrient and carbonate results. EPSL $\underline{49}, 57-80$.

Emerson, S., R. Jahnke, and D. Heggie (1984) Sediment-water exchange in shallow water estuarine sediments. JMR 42, 709-730.

Emrich, K., D.H. Ehalt, and J.C. Vogel (1970) Carbon isotope fractionation during the precip]itation of calcium carbonate. EPSL $\underline{8}, 363-371$.

Ertel, J.R., and J.I. Hedges (1984) The lignin component of humic substances: Distribution among soil and sedimentary humic, fulvic, and base-insoluble fractions. GCA $\underline{48}, 2065-2074$.

Farrington, J.W., S.M. Henrichs, and R. Anderson (1977) Fatty acids and $2:{ }^{\mathrm{Pb}}$ geochronology of a sediment core from Buzzards Bay, MA. GCA $\underline{41}$, 289-296.

Fonselius, (1976) Determination of hydrogen sulfide, in K. Grasshoff, ed. Methods of Seawater Analysis, Verlang Chemie, Weinheim.

Froelich, P.N., G.P. Klinkhammer, M.L. Bender, N.A. Luedtke, G.R. Heath, D. Cullen, P. Dauphin, D. Hammond, B. Hartman, V. Maynard (1979) Early oxidation of organic matter in pelagic sediments of the eastern equatorial Atlantic: suboxic diagenesis. GCA 43, 1075-1091.

Fry, B. and E.B. Sherr (1984) $\delta^{13} \mathrm{C}$ measurements as indicators of carbon flow in marine and freshwater ecosystems. Contributions in Marine Science 27, 13-47.

Gearing, J.N., P.J. Gearing, D.T. Rudnick, A.G. Requejo, M.J. Hutchins (1984) Isotopic variability of organic carbon in a phytoplankton-based estuary. GCA $\underline{48}, 1089-1098$. 
Gilboa-Garber (1971) Direct spectrophotometric determination of inorganic sulfide in biological materials and in other complex materials. Anal. Biochem. 43, 129-133.

Goldhaber, M.B., R.C. Aller, J.K. Cochran, J.K. Rosenfeld, C.S. Martens, R.A. Berner (1977) Sulfate reduction, diffusion, and bioturbation in Long Island Sound sediments. Report of the FOAM group. Am. J. of Sci. 277, 193-237.

Griffin, S., and E.R.M. Druffel (1985) Woods Hole Oceanographic Institution Radiocarbon Laboratory: Sample treatment and gas preparation.

Radiocarbon 27, 43-51.

Grundmanis, V. and J. Murray (1982) Aerobic respiration in pelagic marine sediments. GCA $\underline{46}$, .

Hatcher, P.G., E.C. Spiker, N.M. Szeverenyi, and G.E. Maciel (1983)

Selective preservation and origin of petroleum-forming aquatic kerogen. Nature 305, 498-501.

Henrichs, S.M. (1980) The biogeochemistry of dissolved free amino acids in marine sediments. PhD Thesis, MIT/WHOI WHOI-80-39.

Hedges, J.I., J.R. Ertel, P.D. Quay, P.M. Grootes, J.E. Richey, A.H. Devol, G.W. Farwell, F.W. Schmidt, E. Salati (1986) Organic carbon-14 in the Amazon river system. Science 231, 1129-1131.

Hites, R.A., R.E. Laflamme and J.W. Farrington (1977) Sedimentary polycyclic aromatic hydrocarbons: the historical record. Science 198, 829-831.

Hites, R.A., R.E. Laflamme, J.G. Windsor, J.W. Farrington, W.G. Deuser (1980) Polycyclic aromatic hydrocarbons in an anoxic sediment core from the Pettaquanscutt River (Rhode Island, U.S.A.). GCA 44 , 873-878.

Hough, J.L. (1940) Sediments of Buzzards Bay, Massachusetts. J. of Sed. Petrol. 10, 19-32.

Howarth, R.W. (1984) The ecological significance of sulfur in the energy dynamics of salt marsh and coastal marine sediments. Biogeochem. I, $5-27$.

Ingle, S.E. (1975) Solubility of calcite in the ocean. Mar. Chem. $\underline{3}$, $301-319$.

Jahnke, R., S.R. Emerson, and J.W. Murray (1982) A model of oxygen reduction, denitrification, and organic matter remineralization in marine sediments. L\&O. 
Jahnke, R., D. Heggie, S. Emerson, and V. Grundmants (1982) Pore waters of the central Pacific Ocean: Nutrient results. EPSL 61, 233-256.

Jahnke, R.A., S.R. Emerson, K.K. Roe, and W.C. Burnett (1983) The present day formation of apatite in Mexican continental margin sediments. GCA 49, 259-266.

Johnson, R.W. and J.A. Calder (1973) Early diagenesis of fatty acids and hydrocarbons in a salt marsh environment. GCA 37, 1943-1955.

Jorgensen, B.B. (1977a) The sulfur cycle of a coastal marine sediment (Limfjorden, Den). L \& 0 22, 814-832.

Jorgensen, B.B. (1977b) Bacterial sulfate reduction within reduced microniches of oxidized marine sediments. Mar. Biol. 41, 7-17.

Jorgensen, B.B., and N.P. Revsbech (1985) Diffusive boundary layers and the oxygen uptake of sediments and detritus. L \& 0 30, $111-122$.

Jumars, P.A., A.R.M. Nowell, and R.F.L. Self (1981) A simple Markov model of flow-sediment-organism interaction. Mar. Geol. 42, 155-172.

Kaplan, I.R., and S.C. Rittenberg (1964) Carbon isotope fractionation during metabolism of lactate by Desulfovibrio desulfuricans. J. of Gen. Microbio. 34, 213-217.

Kroopnik, P. (1985) The distribution of ${ }^{13} \mathrm{C}$ of $\left[\mathrm{CO}_{2}\right.$ in the world oceans. DSR $\underline{32}, 57-84$.

Kuenen, J.G., and R.F. Beudeker (1982) Microbiology of thiobacilli and other sulfur-oxidizing autotrophs, mixotrophs, and heterotrophs. Phil. Trans. R. Soc. Lond. B298, 473-497.

Lee, C., R.B. Gagosian, J.W. Farrington (1977) Sterol diagenesis in Recent sediments from Buzzards Bay, MA. GCA 41, 985-992.

Levin, I., B. Kromer, H. Schoch-Fischer, M. Bruns, M. Munsich, D. Berdau, J.C. Vogel, K.O. Munnich (1985) 25 years of tropospheric ${ }^{14} \mathrm{C}$ observation in central Europe. Radiocarbon 27, 1-19.

Li, Y.H., S. Gregory (1974) Diffusion of ions in seawater and in deep-sea sediments. GCA $\underline{38}, 703-714$.

Libes, S.M. (1983) Stable isotope geochemistry of nitrogen in marine particulates. PhD Thesis, WHOI/MIT, WHOI-83-9.

Martens, C.S. and J. Val Klump (1984) Biogeochemical cycling in an organic-rich coastal marine basin 4 . An organic carbon budget for sediments dominated by sulfate reduction and methanogenesis. GCA $\underline{48}$, 1987-2004. 
Martin, W.R. (1985) Transport of trace metals in nearshore sediments. PhD Thesis, WHOI/MIT, WHOI85-18.

McCaffrey, R., A. Myers, Davey et a1. (1980) The relation between pore water chemistry and benthic fluxes of nutrients and $\mathrm{Mn}$ in Narragansett Bay, R.I. L \& $0 \underline{25}, 31-44$.

McCorkle, D.C., S.R. Emerson, and P.D. Quay (1985) Stable carbon isotopes in marine porewaters. EPSL $\underline{74}, 13-26$.

Menzel, D.W. and R.F. Vaccaro (1964) The measurement of dissolved organic and particulate carbon in seawater. Limnol. Oceanogr. 9, 138-142.

Miller, L.G. (1980) Dissolved inorganic carbon isotope ratios in reducing marine sediments. MS thesis, Univ. of South. Calif.

Nissenbaum, A., B.J. Presley, and I.R. Kaplan (1972) Early diagenesis in a reducing fjord, Saanich Inlet, British Columbia I. Chemical and isotopic changes in major components of interstitial waters. GCA $\underline{36}$, 1007-1027.

Penhale, P., and G.W. Thayer (1980) Uptake and transfer of carbon and phosphorous by eelgrass (Zostera marina L.) and its epiphytes. J. Exp. Mar. Biol. Ecol. 42, 113-123.

Peterson, B.J. (1980) Aquatic primary productivity and the ${ }^{14} \mathrm{C}-\mathrm{CO}_{2}$ method: A history of the productivity problem. Ann. Rev. Ecol. Syst. 11, 359-385.

Peterson, B.J., R.W. Howarth, F. Lipschultz, and D. Ashendorf (1980) Salt marsh detritus: an alternative interpretation of stabe carbon isotope ratios and the fate of Spartina alterniflora. OIKOS $34,173-177$.

Presley, B.J., and I.R. Kaplan (1968) Changes in dissolved sulfate, calcium and carbonate from interstitial water of near-shore sediments. GCA $\underline{32}$, 1037-1048.

Reeburgh, W.S. (1983) Rates of Biogeochemical processes in anoxic sediments. Ann. Rev. Earth and Plan. Sci., 269-298.

Reeburgh, W.S. (1982) Amajor sink and flux control for $\mathrm{CH}_{4}$ in marine sediments: Anaerobic Consumption. from The Dynamic Environment of the Ocean Floor, eds. Fanning and Mannheim, Lexington Books, Lexington, MA.

Rhoads, D.C. (1967) Biogenic reworking of intertidal and subtidal sediments in Barnstabe Harbor and Buzzards Bay, MA. Journ. of Geol. 75, 461-476.

Roman, M.R. and K.R. Tenore (1978) Tidal resuspension in Buzzards Bay, MA. ECMS $\underline{6}, 37-46$. 
Rosenfeld, W.D., and S.R. Silverman Carbon isotope fractionation in bacterial production of methane. Science 130, 1658-1659.

Rubinson, M., and R.N. Clayton (1969) ${ }^{13} \mathrm{C}$ fractionation between aragonite and calcite. GCA 33, 997-1002.

Sackett, W.M. (1964) The depositional history and isotopic organic carbon composition of marine sediments. Mar. Geol. 2, 173-185.

Sanders, H.L. (1958) Benthic studies in Buzzards Bay. I. Animal-sediment relationships. L \& $0 \underline{3}, 245-258$.

Sanders, H.L. (1960) Benthic studies in Buzzards Bay. III. The structure of the soft-bottom community. Limnol. and Ocean. $\underline{5}, 138-153$.

Sayles, F.L. (1980) The solubility of $\mathrm{CaCO}_{3}$ in sea water at $2 \mathrm{C}$ based upon in situ sampled pore water composition. Mar. Chem. 9 , 223-235.

Sayles, F.L. (1981) The composition and diagenesis of interstitial solutions-II. Fluxes and diagenesis at the water-sediment interface in the high latitude $\mathrm{N}$. and S. Atlantic. GCA 4 5, 1061-1086.

Scharpenseel, H.W., F. Pietig, and M.A. Tamers (1969) University of Bonn natural radiocarbon measurements II. Radiocarbon 11, 3-14.

Sherr, E.B. (1982) Carbon isotope composition of organic seston and sediments in a Georgia salt marsh estuary. GCA 46, 1227-1232.

Shiller, A.M. and J.M. Gieskes (1980) Processes affecting the oceanic distribution of dissolved $\mathrm{Ca}$ and alkalinity. JGR 85, 2719-2727.

Sholkovitz, E.R., and D.R. Mann (1984) The pore water chemistry of $239,240 \mathrm{Pu}$ and ${ }^{137} \mathrm{Cs}$ in sediments of Buzzards Bay, MA. GCA 48 , $1107-1114$.

Sofer, 2. (1980) Preparation of carbon dioxide for stable carbon isotope analysis of petroleum fractions. Anal. Chem 52, 1389-1391.

Spiker, E.C. and P.G. Hatcher (1984) Carbon isotope fractionation of sapropelic organic matter during early diagenesis. Org. Geochem. $\underline{5}$, 283-290.

Stumm W. and J.J. Morgan (1981) Aguatic Chemistry, Second Edition, John Wiley and Sons, New York.

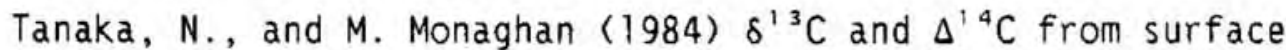
seawater and carbonate tests from Long Island Sound and the western $\mathrm{N}$. Atlantic. EOS 45,964 . 
Turekian, K.K.; Benoit, G.J.; Benninger, L.K. (1980) The mean residence time of plankton derived carbon in a Long Island Sound sediment core: a correction. ECMS 11, 583.

Turekian, K.K. and G.J. Benoit (1981) Radiocarbon in New York Bight sediments and the use of carbon isotopes in delineating carbon sources. in Workshop Rept., Carbon dioxide effects research and assessment program, ed. Comm. of Flux of Organic carbon to the Ocean, CONF-8009140; UC-11.

Turner, J.v. (1982) Kinetic fractionation of ${ }^{13} \mathrm{C}$ during calcium carbonate precipitation. GCA $\underline{46}, 1183-1191$.

Valiela, I., B. Howes, R. Howarth, A. Giblin, K. Foreman, J.M. Teal, J.E. Hobbie (1980) Regulation of primary production and decomposition in a salt marsh ecosystem. from Wetlands: Ecology and Management, eds. B. Gopal, R.E. Turner, R.G. Wetzel, D.E. Whigham.

Vanderborght, J., R. Wollast, and G. Billen (1977) Kinetic models of diagenesis in disturbed sediments. Part $I$. Mass transfer properties and silica diagenesis. L\&O 22, 787-793.

Westrich, J.T. and R.A. Berner (1984) The role of sedimentary organic matter in bacterial sulfate reduction: The $G$ model tested. $L \& 0 \underline{29}$, 236-249.

Whelan, J.K., M.G. Fitzgerald, M. Tarafa (1983) Analyses of organic particulates from Boston Harbor by thermal distillation-pyrolysis. ES\&T 17, 292-298.

Williams, P.M. and T.W. Linick (1975) Cycling of organic carbon in the ocean: use of maturally occurring radiocarbon as a long and short term tracer. IAEA-SM-191/26, 153-167.

Wilson, J.0., I. Valiela, T. Swain (1985) Sources and concentrations of vascular plant material in sediments of Buzzards Bay. Mar. Biol. 90 , 129-137.

Wilson, M.A., R.P. Philp, A.H. Gillam, T.D. Gilbert, K.R. Tate (1983) Comparison of the structures of humic substances from aquatic and terrestrial sources by pyrolysis gas chromatography-mass spectrometry. GCA $\underline{47}, 497-502$. 
APPENDIX I

pata Tables

Pore Water Data

Solid-phase data

Qctober 83

\begin{tabular}{|c|c|c|c|c|c|c|c|c|c|}
\hline$\left.i^{2} \mathrm{~cm}\right)$ & $\begin{array}{l}\delta^{13} \mathrm{C}-\mathrm{SCO}_{2} \\
(0 / 00)\end{array}$ & $\begin{array}{l}\mathrm{pH} \\
-\end{array}$ & $\begin{array}{l}\text { Alk } \\
\text { (mea/L) }\end{array}$ & $\begin{array}{l}\mathrm{Ca} \\
(\mathrm{m}+1)\end{array}$ & $\begin{array}{l}\mathrm{H}_{2} \mathrm{~S} \\
(\mu \mathrm{M})\end{array}$ & $\begin{array}{l}\mathrm{PO}_{4} \\
(\mu M)\end{array}$ & $\begin{array}{l}\mathrm{DOC} \\
(\mathrm{mgc} / \mathrm{L})\end{array}$ & $\% O C$ & $\begin{array}{l}\delta^{13} \mathrm{C}-\mathrm{SOC} \\
(0 / 00)\end{array}$ \\
\hline $\begin{array}{l}0-0.1 \\
0-1 \\
1-2 \\
2-3 \\
3-4 \\
4-6 \\
6-8 \\
8-10 \\
10-12 \\
12-14 \\
14-16 \\
16-18 \\
18-20 \\
20-22 \\
22-24 \\
24-26 \\
26-28 \\
28-30 \\
42-43\end{array}$ & $\begin{array}{l}\text { n.d.A.B } \\
-4.50 \\
-4.71 \\
\text { n.d. } \\
\text { n.d. } \\
-7.70 \\
-7.50 \\
\text { n.d. } \\
\text { n.d. } \\
-4.44 \\
-5.78 \\
\text { n.d. } \\
-6.42 \\
-7.74 \\
\text { n.d. } \\
-9.98 \\
-7.78 \\
-8.89 \\
\text { n.d. }\end{array}$ & $\begin{array}{l}\text { n.d. } \\
7.36 \\
7.16 \\
7.10 \\
7.26 \\
7.34 \\
7.39 \\
7.38 \\
7.30 \\
7.36 \\
7.43 \\
7.45 \\
7.47 \\
7.43 \\
7.46 \\
7.45 \\
7.44 \\
7.42 \\
\text { n.d. }\end{array}$ & $\begin{array}{l}\text { n.d. } \\
3.03 \\
3.40 \\
3.24 \\
4.04 \\
4.45 \\
4.22 \\
4.15 \\
3.79 \\
3.68 \\
3.79 \\
3.91 \\
4.31 \\
4.65 \\
4.86 \\
5.18 \\
4.87 \\
5.62 \\
\text { n.d. }\end{array}$ & $\begin{array}{l}\text { n.d. } \\
\text { n.d. } \\
\text { n.d. } \\
>9.78 \\
9.54 \\
9.38 \\
9.53 \\
\text { n.d. } \\
9.71 \\
\text { n.d. } \\
\text { n.d. } \\
9.49 \\
\text { n.d. } \\
\text { n.d. } \\
\text { n.d. } \\
\text { n.d. } \\
\text { n.d. } \\
\text { n.d. } \\
\text { n.d. }\end{array}$ & $\begin{array}{c}\text { n.d. } \\
\text { n.d. } \\
1 \\
7 \\
\text { n.d. } \\
102 \\
133 \\
\text { n.d. } \\
60 \\
68 \\
78 \\
\text { n.d. } \\
351 \\
\text { n.d. } \\
479 \\
555 \\
584 \\
\text { n.d. } \\
\text { n.d. }\end{array}$ & $\begin{array}{c}\text { n.d. } \\
52 \\
115 \\
44 \\
36 \\
36 \\
28 \\
24 \\
23 \\
19 \\
20 \\
19 \\
20 \\
19 \\
19 \\
16 \\
17 \\
18 \\
\text { n.d. }\end{array}$ & $\begin{array}{l}\text { n.d. } \\
12.8 \\
23.7 \\
\text { n.d. } \\
10.8 \\
19.9 \\
18.4 \\
\text { n.d. } \\
\text { n.d. } \\
\text { n.d. } \\
\text { n.d. } \\
\text { n.d. } \\
29.1 \\
\text { n.d. } \\
\text { n.d. } \\
25.8 \\
\text { n.d. } \\
\text { n.d. } \\
\text { n.d. }\end{array}$ & $\begin{array}{l}2.20 \\
2.08 \\
2.31 \\
2.32 \\
2.25 \\
2.15 \\
2.04 \\
1.99 \\
1.74 \\
1.99 \\
1.90 \\
1.87 \\
1.62 \\
1.65 \\
n . d . \\
1.72 \\
\text { n.d. } \\
1.78 \\
1.64\end{array}$ & $\begin{array}{r}-20.5 \\
-20.8 \\
-20.8 \\
-20.9 \\
-21.0 \\
-20.9 \\
n . d . \\
-21.0 \\
-20.9 \\
-20.8 \\
-20.7 \\
-20.6 \\
-20.6 \\
-20.5 \\
-20.5 \\
-20.4 \\
-20.3 \\
-20.3 \\
-20.3\end{array}$ \\
\hline
\end{tabular}

A -n.d. indicates analysis was not performed on sample

- An asterisk after the value of $\delta^{13} \mathrm{C}-\mathrm{CCO}_{2}$ indicates the sample was sampled to a plastic syringe and filtered into a glass syringe; two asterisks indicates both syringes were glass. 
December 83

$\begin{array}{ll}z & \delta^{13} \mathrm{C}-\sum \mathrm{CO}_{2} \\ (\mathrm{~cm}) & (0 / 00) \\ \mathrm{BW} & 0.4 \\ 0-0.5 & -1.8 \\ 0.5-1 & -3.6,-3.6 \\ i-1.5 & -2.2 \\ 1.5-2 & -2.8 \\ 2-2.5 & -2.8 \\ 2.5-3 & -4.2 \\ 3-3.5 & n . d \\ 3.5-4 & -2.4 \\ 4-5 & -2.0 \\ 6-7 & -4.1 \\ 8-9 & -4.1 \\ 10-11 & -2.9 \\ 15-16 & -3.3 \\ 20-21 & -4.6 \\ 25-26 & -7.2\end{array}$

\begin{tabular}{|c|c|c|c|c|c|}
\hline $\begin{array}{l}\mathrm{SCO}_{2} \\
(\mathrm{mM})\end{array}$ & $\begin{array}{l}\text { A } 1 \mathrm{k} \\
(\mathrm{meg} / \mathrm{L})\end{array}$ & $\begin{array}{l}\mathrm{Ca} \\
(\mathrm{mM})\end{array}$ & $\begin{array}{l}\mathrm{H}_{2} \mathrm{~S} \\
(\mu \mathrm{M})\end{array}$ & $\begin{array}{l}\mathrm{PO}_{4} \\
(\mu M)\end{array}$ & $\begin{array}{l}\mathrm{DOC} \\
(\mathrm{mgC} / \mathrm{L})\end{array}$ \\
\hline 1.97 & 2.22 & 9.16 & - & - & 4.5 \\
\hline 2.50 & 2.44 & $>9.28$ & 1 & 10 & 8.2 \\
\hline 2.63 & 2.65 & n.d. & 2 & 19 & n.d. \\
\hline 2.73 & 2.62 & n.d. & - & 23 & n.d. \\
\hline 2.66 & 2.62 & 9.15 & 2 & n.d. & 15.8 \\
\hline 2.69 & 2.61 & $>9.38$ & 7 & 30 & 13.3 \\
\hline 2.78 & 2.83 & 9.32 & 7 & 43 & 12.5 \\
\hline 2.80 & n.d. & n.d. & 4 & 34 & 13.0 \\
\hline 2.77 & 2.81 & n.d. & 9 & 32 & n.d. \\
\hline 2.82 & 2.89 & 9.42 & 20 & 33 & 8.6 \\
\hline 2.83 & 2.80 & 9.35 & 39 & 26 & 8.5 \\
\hline 2.73 & 2.87 & 9.35 & 31 & 21 & 5.9 \\
\hline 2.79 & 2.90 & 9.30 & 42 & n.d. & 6.2 \\
\hline 2.84 & 2.85 & 9.31 & 42 & 16 & n.d. \\
\hline 3.24 & 3.22 & 9.39 & 118 & 22 & 10.7 \\
\hline 3.70 & 3.84 & 9.37 & 29 & 74 & 12.9 \\
\hline
\end{tabular}

\%OC $\quad \delta^{13} \mathrm{C}-\mathrm{SOC}$

n.d. n.d

$2.87 \quad-20.6$

$3.24 \quad-20.7$

$3.12-20.7$

$2.84 \quad-20.6$

$-20.8$

$2.90 \quad-20.4$

2.89 n.d.

$1.99-20.8$

$1.84-20.3$

$1.94 \quad-20.8$

n.d. n.d.

$1.67-20.7$

$\begin{array}{ll}1.63 & -20.6 \\ 1.68 & -20.4\end{array}$ 
ûd n

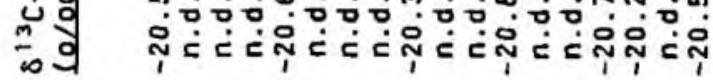

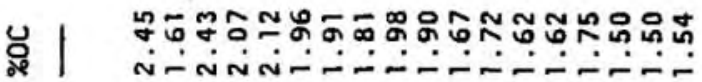

论芶

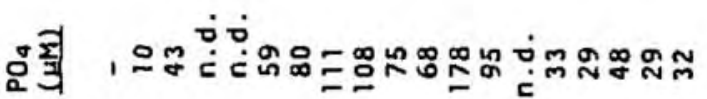

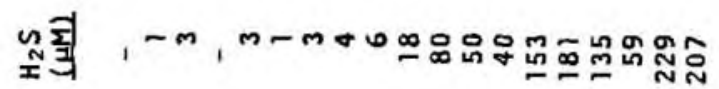

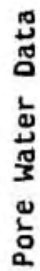

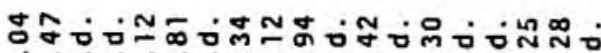

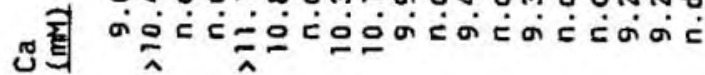

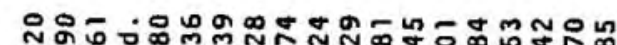

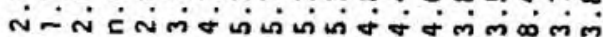

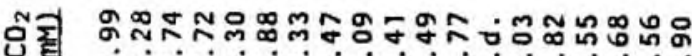

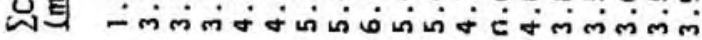

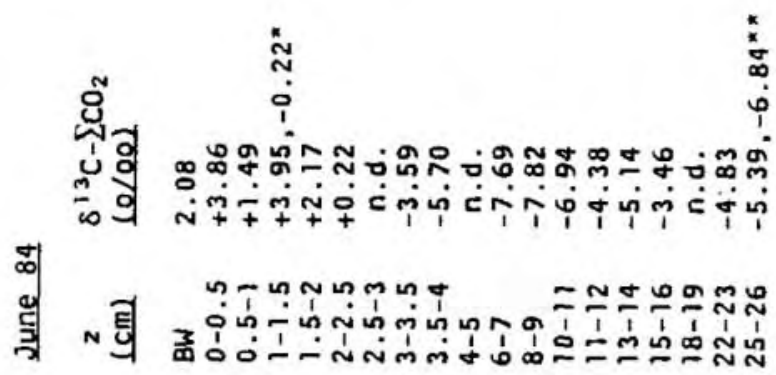


Auqust 84

\begin{tabular}{|c|c|c|c|c|c|c|c|}
\hline $\begin{array}{l}2 \\
(\mathrm{~cm})\end{array}$ & $\begin{array}{l}\delta^{13} \mathrm{C}-\sum \mathrm{CO}_{2} \\
(0 / 00)\end{array}$ & $\begin{array}{l}\mathrm{SCO}_{2} \\
(\mathrm{mM})\end{array}$ & $\begin{array}{l}\text { Alk } \\
(\mathrm{meg} / \mathrm{L})\end{array}$ & $\begin{array}{l}\mathrm{Ca} \\
(\mathrm{mM})\end{array}$ & $\begin{array}{l}\mathrm{H}_{2} \mathrm{~S} \\
(\mu M)\end{array}$ & $\begin{array}{l}\mathrm{PO}_{4} \\
(\mu \mathrm{M})\end{array}$ & $\% 0 \mathrm{C}$ \\
\hline $\begin{array}{l}B W \\
0-0.5 \\
0.5-1 \\
1-1.5 \\
1.5-2 \\
2-2.5 \\
2.5-3 \\
3-3.5 \\
3.5-4 \\
5-6 \\
7-8 \\
9-10 \\
11-12 \\
13-14 \\
15-16 \\
19-20 \\
24-25 \\
29-30\end{array}$ & $\begin{array}{l}+1.29 \\
\text { n.d. } \\
-2.46 \\
-2.10,-2.24^{*} \\
-4.64 \\
-2.27 \\
-4.66 \\
-5.22 \\
-5.08 \\
\text { n.d. } \\
-7.41 \\
\text { n.d. } \\
-7.69^{\star} \\
-7.13 \\
-7.74 \\
-7.08 \\
-8.90 \\
-9.65\end{array}$ & $\begin{array}{l}2.01 \\
3.10 \\
3.36 \\
3.58 \\
3.70 \\
3.59 \\
3.79 \\
3.96 \\
4.03 \\
4.26 \\
4.17 \\
4.37 \\
4.51 \\
4.58 \\
4.38 \\
4.46 \\
4.90 \\
5.41\end{array}$ & $\begin{array}{l}2.22 \\
2.55 \\
2.46 \\
2.36 \\
2.51 \\
2.59 \\
2.90 \\
3.23 \\
3.35 \\
4.13 \\
4.10 \\
4.46 \\
4.30 \\
4.40 \\
4.17 \\
n .0 . \\
4.24 \\
5.29\end{array}$ & $\begin{array}{l}\text { n.d. } \\
9.28 \\
\text { n.d. } \\
9.35 \\
9.29 \\
9.33 \\
9.38 \\
>9.37 \\
9.34 \\
9.36 \\
9.35 \\
\text { n.d. } \\
9.40 \\
9.33 \\
\text { n.d. } \\
\text { n.d. } \\
\text { n.d. } \\
\text { n.d. }\end{array}$ & $\begin{array}{r}- \\
0 \\
0 \\
1 \\
1 \\
2 \\
2 \\
4 \\
5 \\
10 \\
146 \\
\text { n.d. } \\
417 \\
480 \\
469 \\
722 \\
668 \\
1066\end{array}$ & $\begin{array}{l}- \\
17 \\
59 \\
91 \\
105 \\
104 \\
96 \\
88 \\
79 \\
66 \\
39 \\
\text { n.d. } \\
33 \\
32 \\
29 \\
30 \\
24 \\
\text { n.d. }\end{array}$ & $\begin{array}{l}2.42 \\
\text { n.d. } \\
\text { n.d. } \\
2.18 \\
\text { n.d. } \\
\text { n.d. } \\
\text { n.d. } \\
2.15 \\
2.04 \\
\text { n.d. } \\
\text { n.d. } \\
\text { n.d. } \\
\text { n.d. } \\
1.62 \\
\text { n.d. } \\
1.75 \\
1.67\end{array}$ \\
\hline
\end{tabular}


Pore Water Data

March 85

\begin{tabular}{|c|c|c|c|c|c|c|c|}
\hline$\stackrel{2}{(\mathrm{~cm})}$ & $\begin{array}{l}\delta^{13} \mathrm{C}-\mathrm{SCO}_{2} \\
(0 / 00)\end{array}$ & $\begin{array}{l}\mathrm{SCO}_{2} \\
(\mathrm{mMl})\end{array}$ & $\begin{array}{l}\text { Alk } \\
\text { (mea/L) }\end{array}$ & $\begin{array}{l}\mathrm{Ca} \\
(\mathrm{mM})\end{array}$ & $\begin{array}{l}\mathrm{H}_{2} \mathrm{~S} \\
(\underline{M}) \mathbf{M})\end{array}$ & $\begin{array}{l}\mathrm{PO}_{4} \\
(\mu \mathrm{M})\end{array}$ & YOC \\
\hline $\begin{array}{l}\text { BW } \\
0-0.5 \\
0.5-1 \\
1-1.5 \\
1.5-2 \\
2-2.5 \\
2.5-3 \\
3-4 \\
6-7 \\
9-10 \\
12-13 \\
14-15 \\
18-19 \\
24-25 \\
29-30\end{array}$ & $\begin{array}{c}0.0 \\
-3.58 \\
-3.19 \\
-1.51 \\
-1.32 \\
-0.16 \\
-0.72 \\
-0.27 \\
\text { n.d. } \\
-4.69 \\
-6.80 \\
\text { n.d. } \\
\text { n.d. } \\
-9.76 \\
\text { n.d. }\end{array}$ & $\begin{array}{l}n . d . \\
3.05 \\
3.01 \\
2.87 \\
3.00 \\
3.13 \\
2.93 \\
3.00 \\
n . d . \\
4.03 \\
4.03 \\
3.70 \\
3.89 \\
3.91 \\
3.97\end{array}$ & $\begin{array}{l}\text { n.d. } \\
3.06 \\
2.96 \\
2.87 \\
2.56 \\
2.50 \\
2.46 \\
2.52 \\
3.65 \\
4.02 \\
4.11 \\
4.01 \\
3.96 \\
3.99 \\
4.14\end{array}$ & $\begin{array}{r}9.47 \\
>9.99 \\
\text { n.d. } \\
>10.32 \\
\text { n.d. } \\
\text { n.d. } \\
\text { n.d. } \\
>10.79 \\
\text { n.d. } \\
<9.95 \\
\text { n.d. } \\
\text { n.d. } \\
\text { n.d. } \\
\text { n.d. } \\
\text { n.d. }\end{array}$ & $\begin{array}{r}- \\
1 \\
3 \\
3 \\
3 \\
5 \\
4 \\
2 \\
10 \\
59 \\
155 \\
170 \\
231 \\
274 \\
292\end{array}$ & $\begin{array}{l}- \\
1 \\
8 \\
10 \\
11 \\
24 \\
18 \\
35 \\
35 \\
37 \\
n . d . \\
31 \\
31 \\
28 \\
34\end{array}$ & $\begin{array}{l}2.23 \\
2.30 \\
\text { n.d. } \\
\text { n.d. } \\
\text { n.d. } \\
2.10 \\
2.11 \\
2.05 \\
1.88 \\
\text { n.d. } \\
\text { n.d. } \\
1.58 \\
\text { n.d. } \\
1.56\end{array}$ \\
\hline
\end{tabular}

Solid-phase data 
Pore Water Data

October 85

\begin{tabular}{|c|c|c|c|c|c|}
\hline $\begin{array}{c}2 \\
(\mathrm{~cm})\end{array}$ & $\begin{array}{l}\delta^{13} \mathrm{C}-\sum \mathrm{CO}_{2} \\
(0 / 00)\end{array}$ & $\begin{array}{l}\mathrm{SCO}_{2} \\
(\mathrm{mM})\end{array}$ & $\begin{array}{l}\text { A } 7 \mathrm{k} \\
\text { (meg/L) }\end{array}$ & $\begin{array}{l}\mathrm{PO}_{4} \\
(\mu M)\end{array}$ & $\% \mathrm{OC}$ \\
\hline $8 W$ & -2.12 & 1.99 & 2.17 & - & \\
\hline $0-0.5$ & -6.20 & 2.37 & 2.46 & 7 & 2.17 \\
\hline $0.5-1$ & -4.62 & 2.42 & 2.36 & 28 & 1.64 \\
\hline $1-1.5$ & -4.04 & 2.42 & 2.29 & 16 & 2.42 \\
\hline 1. $5-2$ & -4.68 & 2.65 & 2.45 & 42 & 2.00 \\
\hline $2-2.5$ & -5.85 & 2.62 & 2.56 & 51 & 2.15 \\
\hline $2.5-3$ & -5.91 & 2.64 & 2.61 & 42 & 2.10 \\
\hline $3-4$ & n.d. & 2.67 & 2.79 & 31 & 1.93 \\
\hline $6-7$ & -6.42 & 2.69 & 2.72 & 35 & 1.83 \\
\hline $9-10$ & -5.37 & 2.58 & 2.71 & 36 & 1.84 \\
\hline $12-13$ & -5.82 & n.d. & 2.68 & 26 & 1.75 \\
\hline $14-15$ & -7.93 & 2.46 & 2.56 & 15 & 1.70 \\
\hline $18-19$ & -5.08 & 2.56 & 2.65 & 17 & 1.60 \\
\hline $24-25$ & -7.31 & 2.72 & 2.79 & 21 & 1.58 \\
\hline $29-30$ & & 3.54 & 3.21 & 28 & 1.68 \\
\hline
\end{tabular}

\section{Solid-phase data}




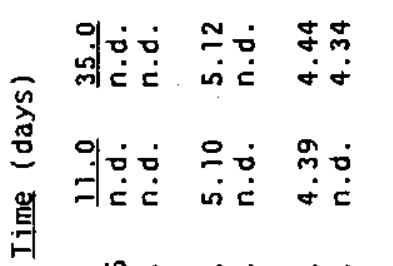

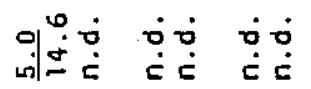

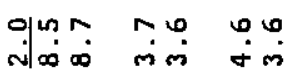

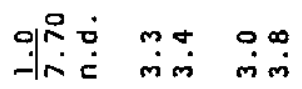

불

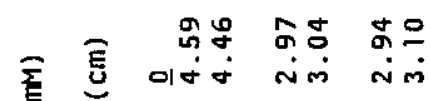

总

包㶽

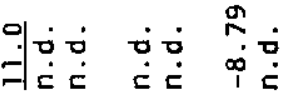

n

管

搹

भौํ்

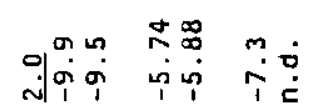

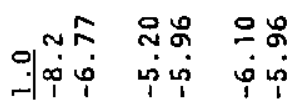

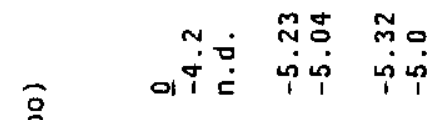

을

岕

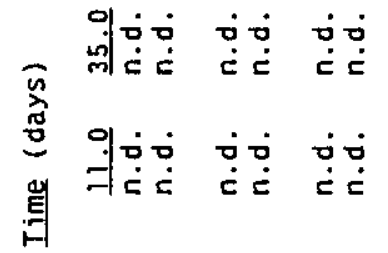

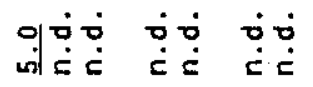

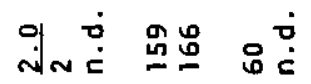

기 움 m요

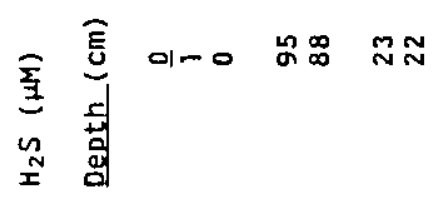

भीज्ञ

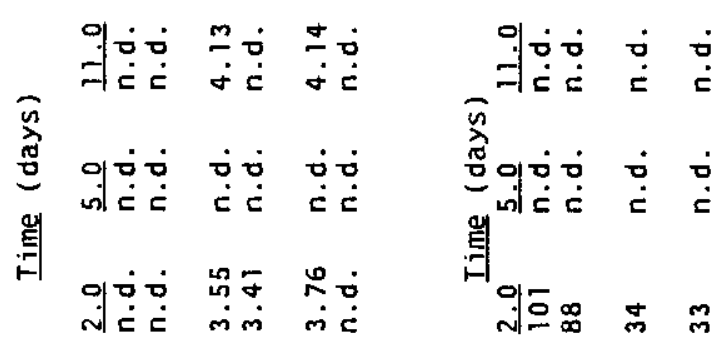

团察它

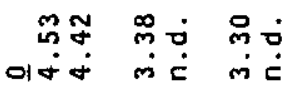

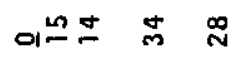

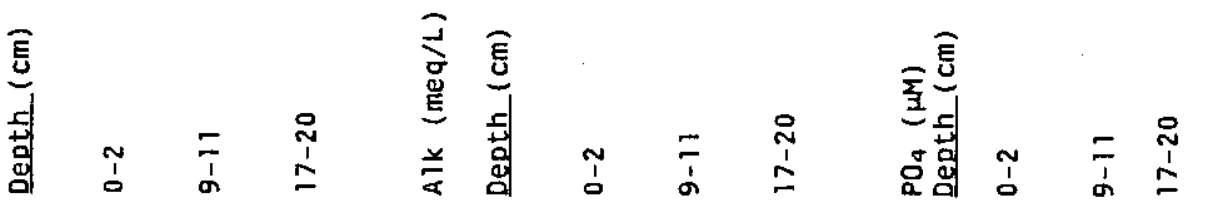




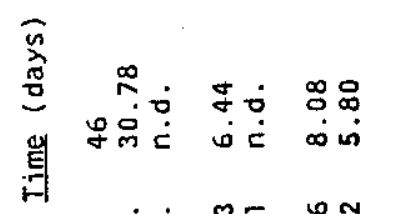

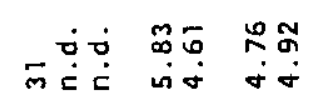

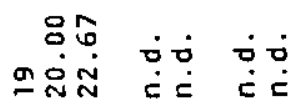

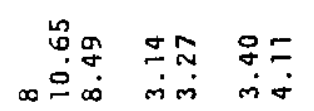

స్

氙 利

m

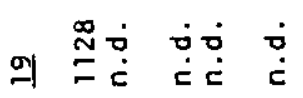

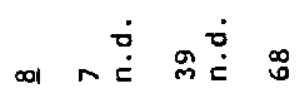

⿹ㅚ

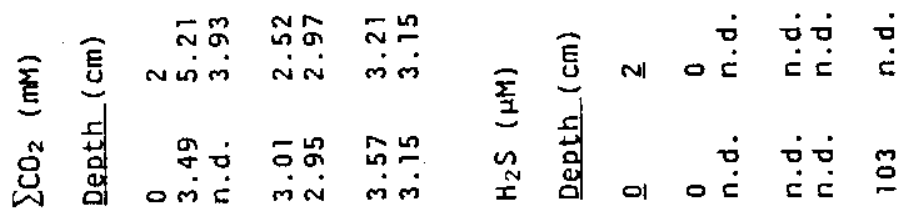

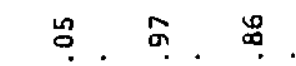

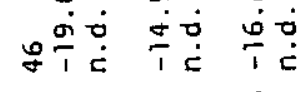

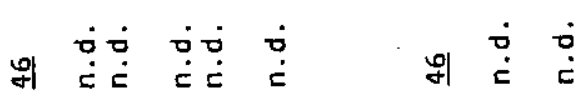

㿟宅

ते

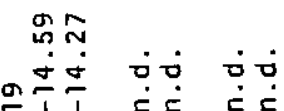

哃

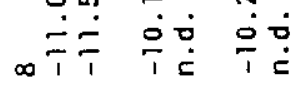

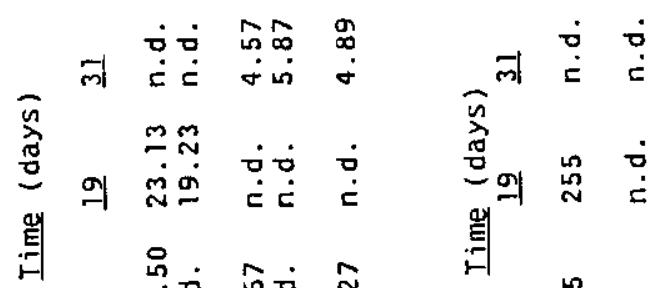

N

$\infty$ ஹீ:

$\infty \stackrel{\leftrightarrow}{\stackrel{0}{\sim}}$

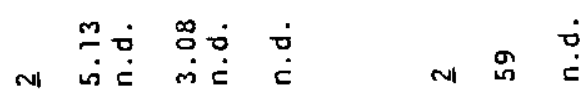

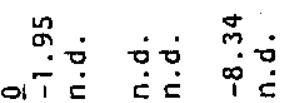

ol

of $\stackrel{\infty}{\stackrel{0}{0}}$

응

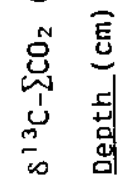

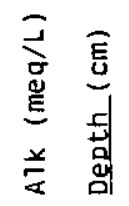

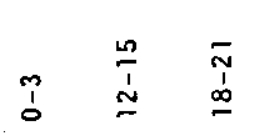

豆言

$\stackrel{\frac{1}{1}}{0} \stackrel{\frac{1}{\sim}}{0}$ 


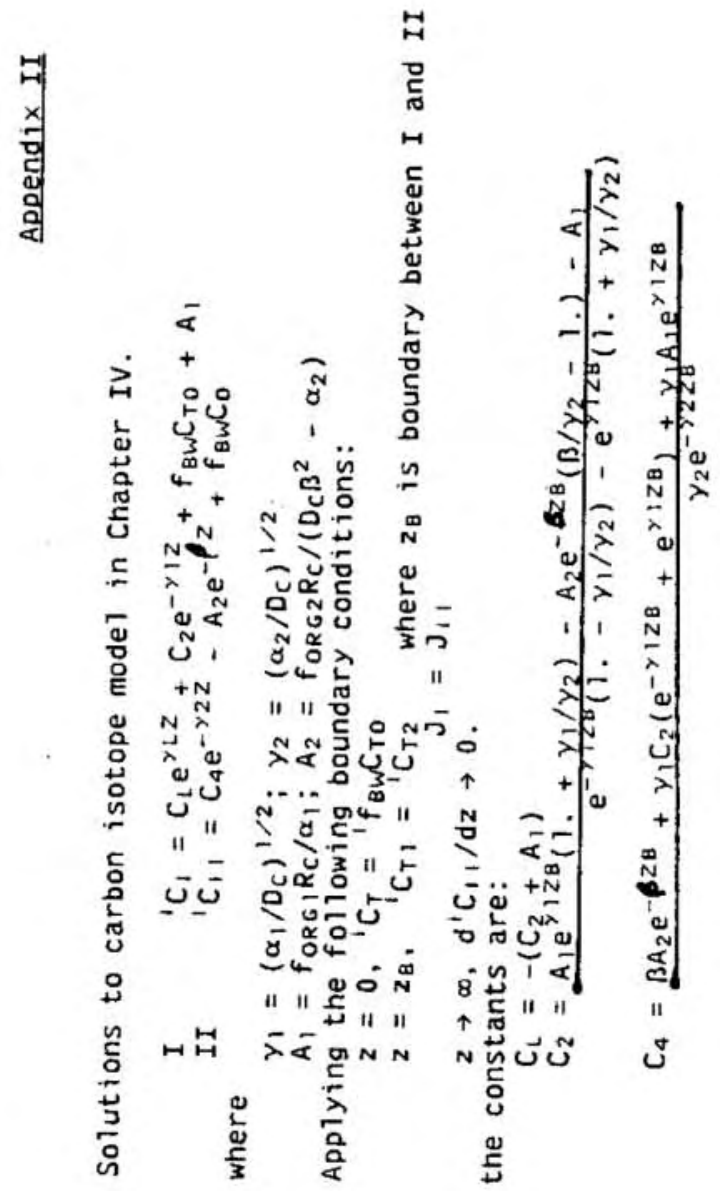




\section{BIOGRAPHICAL NOTE}

The author was born in Bryn Mawr, Pennsylvania on October 29, 1956. She attended Our Lady of the Assumption grade school, Country Day School of the Sacred Heart high school, and Trinity Colege in Hartford, Connecticut. She was graduated with a B.S. in chemistry from Trinity College in May, 1978. She worked at the Rockefeller University in New York, New York for almost two years before entering the Joint Program in June, 1980.

\section{Publications}

Hunt, J.M. and A.P. McNichol (1984) The Cretaceous Austin Chalk of South Texas--A petroleum source rock. in Petroleum Geochemistry and Source Rock Potential of Carbonate Rocks, J.G. Palacas, ed. AAPG Studies in Geology \#18, p. $117-125$. 